
Mining the human intestinal microbiota for biomarkers associated with metabolic disorders

\author{
Gerben Hermes
}




\section{Thesis committee}

\section{Promotor}

Prof. Dr H. Smidt

Personal Chair at the Laboratory of Microbiology

Wageningen University

\section{Co-promotor}

Dr E.G. Zoetendal

Associate professor, Laboratory of Microbiology

Wageningen University

\section{Other members}

Prof. Dr B.J.M. Witteman, Wageningen University

Dr A. Mercenier, Nestlé, Lausanne, Switzerland

Dr A. Zhernakova, M.D., University Medical Centre Groningen

Dr W. van Schaik, University Medical Centre Utrecht

This research was conducted under the auspices of the Graduate School VLAG

(Advanced studies in Food Technology, Agrobiotechnology, Nutrition and Health Sciences) 


\title{
Mining the human intestinal microbiota for biomarkers associated with metabolic disorders
}

\author{
Gerben Hermes
}

Thesis

submitted in fulfilment of the requirements for the degree of doctor at Wageningen University

by the authority of the Rector Magnificus

Prof. Dr A.P.J. Mol,

in the presence of the

Thesis Committee appointed by the Academic Board to be defended in public on Friday 9 December 2016 at 1.30 p.m. in the Aula. 


\section{Gerben Hermes}

Mining the human intestinal microbiota for biomarkers associated with metabolic disorders.

205 pages

$\mathrm{PhD}$ thesis, Wageningen University, Wageningen, NL (2016)

With references, with a summary in English

ISBN: 978-94-6257-951-4

DOI: $10.18174 / 391731$ 


\section{Table of contents}

Chapter 1. General introduction \& thesis outline 7

Chapter 2. Molecular ecological tools to decipher the role 27 of our microbial mass in obesity

Chapter 3. Gut microbiota and body weight in healthy school- 55 aged children: The KOALA Birth Cohort Study

Chapter 4. Effects of gut microbiota manipulation by antibiotics on host metabolism in obese humans: a randomized double-blind placebo-controlled trial

Chapter 5. Limited, individual- and cohort-specific patterns of gut microbiota composition associated with tissue-specific insulin sensitivity in overweight and obese males

Chapter 6. NG-Tax, a highly accurate and validated pipeline for the analysis of $16 \mathrm{~S}$ rRNA amplicons from complex biomes

Chapter 7. Dynamics of microbial community composition and

Chapter 8. General discussion \& Summary

Appendices 



\section{Chapter 1}

General introduction \& thesis outline 


\section{Introduction}

All through most of human history we have predominantly felt at war with microorganisms. Small pox, measles, bubonic plague, lepra, cholera, typhoid are just a few important examples of viral and bacterial agents that have been mostly eliminated in the Western world, while modern day infectious diseases include malaria (protozoan) and HIV/AIDS (viral), and worldwide tuberculosis (bacterial) still causes nearly 2 million deaths every year. The field of microbiology developed in response to the need to conquer these pathogens and eradicate infectious diseases from society, which led to important discoveries such as Louis Pasteur's "germ theory of disease". But a new understanding is emerging in which the metaphor for war and quote from Paul Verhoeven's Starship troopers "the only good bug is a dead bug" is no longer appropriate, as this approach ignores the community context of infectious disease. Instead, we now view the host and its microbial community as a mutualistic co-evolved system. In fact, microorganisms are everywhere and the vast majority of those that we come into contact with are not infectious, but rather harmless or even beneficial. For instance, microorganisms help the host with digestion by degrading non-digestible plant polysaccharides, which are converted into Short Chain Fatty Acids (SCFA) that provide energy for our metabolism. More importantly, the SCFA butyrate is the main source of energy for the intestinal epithelium in mammals ${ }^{1}$.

However, the idea that there actually might be beneficial bacteria is already over a century old. At the beginning of the $20^{\text {th }}$ century, immunologist Ilya Ilyich Metchnikoff in his essays, The Nature of Man: Studies in Optimistic Philosophy (1903) and The Prolongation of Life: Optimistic Studies (1907) theorized that colonic microbial inhabitants released toxins that were dispersed throughout the host, and phagocytes destroyed those toxins. However, this process also caused collateral damage to the host, and therefore the replacement of our native colonic inhabitants with host-friendly bacteria found in yoghurt, might promote health and longevity, so that senility could be delayed and society governed by the wise elderly, rather than the childish and silly young. His theory flourished for a time, then drifted to the fringes of medical practice ${ }^{2}$.

However, the concept of host-friendly bacteria was revived in the mid-1990s as a concept worthy of mainstream medical attention. With it also came the first wave of molecular techniques that mainly made use of the 16S ribosomal RNA (rRNA) gene, such as random cloning and sequencing, Temperature/Denaturing Gradient Gel Electrophoresis, (TGGE, DGGE), quantative PCR (qPCR) of 16S rRNA genes, as well as Fluorescent In Situ Hybridization (FISH) coupled to flow cytometry or microscopy for quantification, to bypass the need for culturing the actual microbes. Before that, the identification of individual members and characterization of microbial ecosystems mostly depended on the ability to culture those bacteria. The first cultured representative from the human gastrointestinal (GI) tract was Escherichia coli, isolated by Theodor Escherich in 1885, 
naming it Bacterium coli commune. Application of the molecular tools mentioned above revealed that the largest fraction of micro-organisms from the GI ecosystem is still uncultured ${ }^{3}$.

Due to the relatively young age of the research field and the concomitant exponential increase in throughput and cost effectiveness of the analytical approaches to study the GI microbes at various levels (DNA,RNA, proteins and metabolites), combined with increased computational power, our understanding of this highly complex ecosystem is expanding at a very high rate. Nevertheless, as a result of this higher cost-effectiveness and interest in the research field, the generation of compositional data is currently also shifting to nonspecialist labs. Therefore, there is quite some confusion regarding the terminology used to describe the micro-organisms in the GI-tract. This thesis will use the terminology proposed by Marchesi et al. for this specific ecosystem ${ }^{4}$, but their definitions in fact apply to all defined environments. The microbes in our GI-tract are collectively called the microbiota ("small life"), although generally only the bacterial fraction is considered. The metagenome describes the combined genetic content of the microbiota (yet the term metagenomics is often incorrectly applied to methodologies only utilizing the 16S rRNA gene) and microbiome refers to all the biotic factors (in the case of the human GI tract this includes bacteria, archeaea, eukarya and viruses), their genomic content and abiotic factors as well. Actually, the term applies to all life forms in a habitat, but it is often used to describe the bacterial metagenomic fraction only. Finally, the incorrect term microflora, which has a longstanding history in medical and scientific literature, is confusing and outdated, as it refers to either 'microscopic plant life' or 'all plant life in a microhabitat'. Although, due to its familiarity for the general public it serves some educational purpose, medical professionals and scientists should refrain from using the term and use microbiota or microbiome instead, depending on what they want to describe. As this thesis focuses in the bacterial fraction of the human GI microbiome, all the aforementioned terms will apply only to the bacterial portion.

\section{Tools of the trade}

Because we still cannot culture the vast majority of the bacteria in this ecosystem we must assess the composition, function, activity and output of the system through indirect means by studying bacterial DNA (metagenomics), RNA (metatranscriptomics), proteins (metaproteomics) and metabolites. Within the DNA based methods we can distinguish between methods that asses the whole genomic content or through PCR amplification of specific genes, such as the 16S rRNA gene. All technologies have their respective advantages and disadvantages associated with information obtained versus costs and accuracy, phylogenetic depth and analytical complexity, yet they are complementary (Table 1). Depending on the research questions asked, a combination of technologies can be 
employed to gain maximum insight into who is doing what, with whom and at what moment.

Table 1. The information output of tools to study the composition, function and activity of the microbiota at the level of DNA, RNA and metabolites.

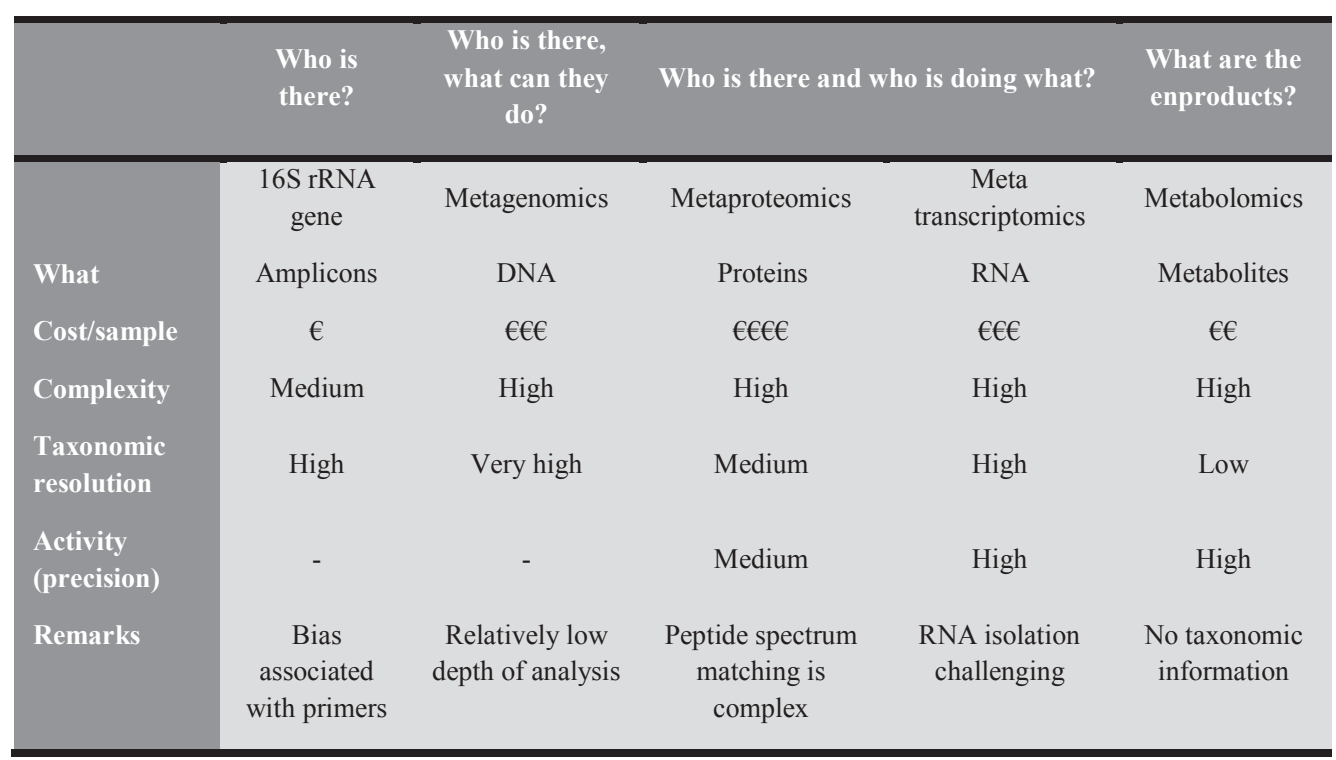

\section{The human GI microbiome in numbers}

If we applied the popular and actually quite appropriate term 'super-organism' to the combination of the host and its microbiome, we could underscore the importance of the microbial fraction of this ecosystem with sheer numbers. The ratio of human cells to microbial cells is now estimated to be between $1 / 1$ to $1 / 3$ depending on the number of red blood cells $\left(\sim 10^{14}-10^{13}\right)^{5}$, however, regarding non-redundant genes this ratio is closer to $1 / 100$ in favour of the microbes. Several gut microbial gene catalogues have been published since 2010, which saw the number of genes expanding from 3.3 million $^{6}$ to 7.4 million $^{7}$ and 9.8 million in $2014^{8}$, which is a vast genetic functional reservoir, compared to our $\sim 20.000$ genes. The number of bacterial strains in an individual is now estimated to be $195 \pm 48$, representing $101 \pm 27$ species $^{9}$.

\section{The formation of a gut microbiome}

Recently, culture based and culture independent studies have questioned the idea that the uterus is sterile, and it has been suggested that bacteria are present in the placenta ${ }^{10}$, amniotic fluid $^{11}$, meconium ${ }^{12}$ and umbilical cord blood ${ }^{13}$. However, these studies generally lacked true biological and technical controls. Biological controls should in these cases 
probably include maternal blood, as both vaginal and C-section delivery are usually associated with mild levels of bacteraemia ${ }^{14}$. Technical controls, especially for samples with low microbial biomass have been shown to be often dominated by bacterial DNA contamination of commercial reagents, dust, and other sources ${ }^{15-17}$. Therefore, further studies are necessary to unequivocally confirm the existence of a viable intra-uterine microbiome and whether this might affect the development of the newborn ${ }^{18}$. It is generally accepted that the first major exposure to microbes is during the birthing process and is highly dependent of mode of delivery. The first inoculation during natural childbirth clearly resembles the maternal vaginal microbiota, with input from the other parts of the urogenital tract and likely the fecal microbiota as well. In contrast, infants delivered through a Caesarean section (C-section) are colonized with common skin and environmental microbes ${ }^{18}$ (Figure 1). Although the difference in microbiota composition between children born vaginally or by $\mathrm{C}$-section seems to gradually decrease, $\mathrm{C}$-section delivery has been associated with delayed colonization of certain taxa ${ }^{19}$. Furthermore, C-section delivery has been associated with several diseases in epidemiological research ${ }^{20}$, although the causality of these relations remains to be demonstrated. The initial inoculum initiates a succession of events leading to the development of a child's own microbiome. In this dynamic process the microbial abundance increases from zero at birth to over six orders of magnitude within the first few weeks of life, with large fluctuations in the microorganisms present and their relative abundance. Diversity generally increases, aerobes are succeeded by facultative and then strict anaerobes and, roughly up until the introduction of the first solid foods, a well constrained range of stereotypical bacteria emerge in the faeces (Figure 1). Random Forests-derived sparse models generated from different datasets from infants/children from very distinct cultural background and geographical locations including Malawi ${ }^{21,22}$, Bangladesh ${ }^{23}$ and the $\mathrm{USA}^{24}$, and whose changes in relative abundance over time defined a program of normal maturation of the microbiota from healthy individuals across biologically related and unrelated individuals, revealed 25 age-discriminatory bacterial groups, that could be used to determine the maturity of the microbiota. Comparison of these models from the different cohorts revealed some overlap in species, especially related to Bifidobacterium and Faecalibacterium. Furthermore, application of the models across datasets showed that the USA model performed consistently well across the three populations (Spearman's correlation coefficients of 0.73 and 0.78 in the non-Western datasets), whereas the application of the other models to the other datasets showed high concordance between the non-Western datasets, but not with the USA cohort. This could imply that a large portion of the taxa from the Western cohort was found in the non-Western cohorts, but not vice versa, pointing at a higher phylogenetic diversity in the non-Western cohorts, but overall similar temporal dynamics.

Exclusive breast-feeding generally selects for genera specialized in the utilization of complex human milk oligosaccharides, such as Bifidobacterium ${ }^{25}$ and to a lesser extent 
Bacteroides as they can compete for the same ecological niche ${ }^{26}$ (Figure 1). During the first months the composition of the microbiota is highly dynamic within individuals and different between individuals and characterised by large abrupt community shifts with interludes of relative stability of varying lengths of time ${ }^{27-30}$. Sometimes these shifts occur together with life events that likely instigate considerable environmental pressure, such as antibiotics use, fever and formula feeding ${ }^{30}$. Palmer and co-workers even observed synchronized transitions in the one pair of dizygotic twins that this study contained, compared to the unrelated individuals, which suggests that shared exposures and/or genetics play an important role during this phase $\mathrm{e}^{30}$.

Abrupt shifts in composition reflect changes in filtering by the host, such as gut mucosal IgA responses ${ }^{24}$ and possibly opportunistic invasions by better-adapted species ${ }^{31}$. Finally, an infant's unique developmental path through this early unstable phase may have longerterm health implications ${ }^{18}$. The introduction of solid foods and weaning are generally associated with the onset of a transition towards an adult-like GI microbiome until the microbiota largely stabilizes in membership after approximately three years of life Figure $1^{18}$.

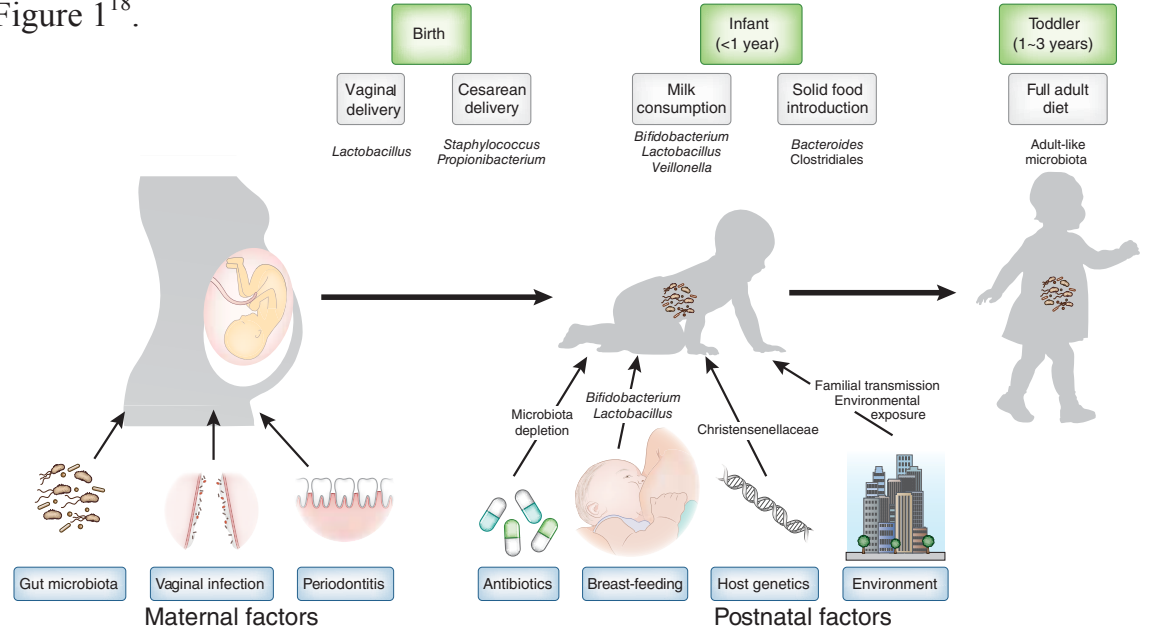

Figure 1. Factors shaping the neonatal microbiome ${ }^{18}$.

At the same time, the GI microbiota triggers the development and maturation of the newborn's immune system. Although there is still a great deal of research needed to understand precisely what happens in this developmental process, it appears the maturing immune system relies on the presence of microbial communities, and especially the presence of these early microbes, to distinguish "self" from "non-self". The significance of bacteria in these early stages of life can be indirectly inferred from germ free animals, that express dramatic alterations in practically every phenotype that has been studied, including the immune system, brain development, metabolism, and the function of the heart, lungs, 
and lymph nodes ${ }^{32}$. It seems that the early colonizers play a large role in shaping our immune system and might even have been drivers for the development of adaptive immunity. Typically, evolutionary pressures that shape immune strategies are often viewed from a host defence perspective. However, it has been proposed that the invention of adaptive immunity could well have been driven as a means to promote, rather than limit, microbial colonization. In this view, organisms that could harness the more flexible metabolic capabilities of a permanent and diverse GI microbiota would have been given a considerable advantage, while mitigating the resultant risk of infections ${ }^{33}$.

Eventually the complete GI tract is colonized in cell densities that increase from $<10^{4} / \mathrm{g}$ in the stomach to $10^{10-11} / \mathrm{g}$ of feces in the colon. Most likely influenced by strong environmental selection (e.g. $\mathrm{pH}$, diet, gut transit time), composition, diversity and function seem to be compartment $\operatorname{specific}^{34}$ (Figure 2). In this thesis the fecal microbiota was used as a proxy for the colonic microbiota.

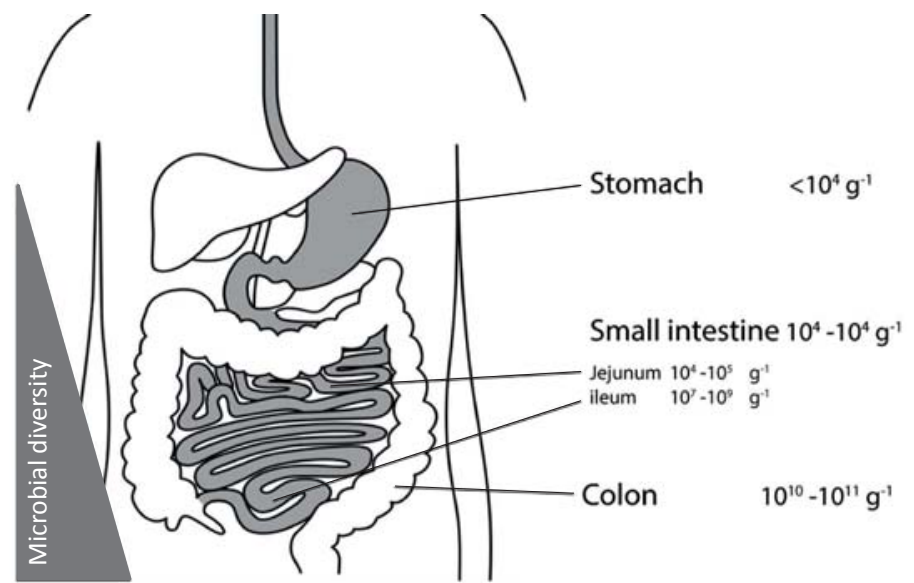

Figure 2. Schematic representation of the microbial density and diversity along the human GI tract.

\section{Inter-individual variability}

At the higher taxonomic ranks, membership of the human GI microbiota is quite similar and mainly consists of species from the phyla Firmicutes, Bacteroidetes, Actinobacteria, Verrucomicrobia and Proteobacteria. At the species and strain level, however, each individual seems to be host to a unique and almost personal microbiota. The previous section has highlighted the processes that could possibly give rise to such variation. This section will focus on the role of another variable in this complex multifactorial process of shaping the intra-individual variation of the microbiota: namely the host itself.

Currently, based on well powered studies, combined with relatively deep interrogation of the fecal microbiota, there is no consensus with regards to the heritability of the microbiota or components thereof. Heritability studies rely on twins to reveal the relative importance 
of environmental and genetic influences on individuals. In human studies of heritability these are often apportioned into factors from "shared environment" and "non-shared environment" based on the premise that persons brought up in the same household share an environment that is more or less similar compared to persons who were not. The classical twin design compares the similarity of monozygotic (identical) and dizygotic (fraternal) twins. If identical twins are considerably more similar than fraternal twins, this implicates that host genotype plays an important role in these traits. By comparing several hundreds of families of twins, researchers can then understand more about the roles of genetic effects, shared environment, and unique environment in shaping the gut microbiome.

Several studies have reported that the microbiota of monozygotic twin pairs is no more similar in terms of their gut bacterial community structure than are similarly aged dizygotic co-twins at several age ranges from very different cultural backgrounds and geography. These studies included individuals from the Amazonas of Venezuela, rural Malawi, Bangladesh and the US metropolitan areas with Hispanic, non-Hispanic and African American twins, living together as well as apart, throughout the United States. Studies covered the complete spectrum of developmental progression of the microbiota. The early years: 1-12 months ${ }^{35}, 0-24$ months $^{24}, 0-36$ months $^{21}$ and the transition into adulthood: teenaged twins ${ }^{35}$ and finally adults ${ }^{36}$. Most likely, differences in social structures may influence the extent of vertical transmission of the microbiota and the flow of microbes and microbial genes among members of a household. Differences in cultural traditions also affect food, exposure to pets and livestock, and many other factors that could influence how and from where a member of the gut microbiome is acquired. These studies revealed that the fecal microbiota of mothers were no more similar to their children than were those of the biological fathers, and that genetically unrelated but co-habiting mothers and fathers were significantly more similar to one another microbially than were members of different families. Remarkably the overall patterns of the effects of kinship on microbial community structure were found to be similar between individuals from very distinct geographical and cultural areas, suggesting the underlying mechanisms for the degree of similarity among members of a family are relatively universal. These findings emphasize the significance of a history of numerous common environmental exposures and that we acquire microbes from competing sources other than, or in addition to, our family members, which might indicate strong selection for an individualized microbiota that is largely influenced by personal exposure history.

Nevertheless, a study with 416 twin pairs from the UK reported a heritable component of the microbiota, by showing that membership (i.e. MZ twins had more taxa in common) but not the overall structure showed significant differences between MZ and DZ twins ${ }^{37}$. They also showed that only some components of the microbiota were heritable. For instance from the three most abundant bacterial families Ruminococcaceae, Lachnospiraceae and Bacteroidaceae, only the latter didn't show a difference in abundance between MZ and DZ 
twin-pairs. Although this seems to point to the influence of genetics, this conclusion depends on the validity of the "equal environments assumption" (EEA) as an underlying premise, and which assumes that identical and fraternal twin pairs experience equivalent trait-relevant environmental exposures, especially early in life. However, lately this assumption has been shown to be flawed, and hence its use does not provide any valid indication of genomic effects ${ }^{38,39}$.

Nonetheless, several genes have been associated with differences in microbiota composition in smaller case-control studies. These include, for example, the FUT2 gene that codes for the fucosyltransferase 2 enzyme, which is responsible for the synthesis of the $\mathrm{H}$ antigen in body fluids and on the intestinal mucosa. The $\mathrm{H}$ antigen is an oligosaccharide moiety that acts as both an attachment site and carbon source for intestinal bacteria ${ }^{40}$. Another gene, Nod2, is involved in GI homeostasis, and has been shown to impact microbiota composition in rodents ${ }^{41}$. Interestingly, certain polymorphisms of both of these genes have been implicated in the etiology of IBD and an increased risk for other pathologies, ${ }^{40}$ while all aforementioned twin studies consisted of healthy individuals. This could point to a stronger host-genotype effect on the microbiota in certain GI disorders.

\section{Intra individual variability}

Although short term intra-individual changes are less pronounced than inter-individual changes $^{36,37,42}$, describing the adult state as 'stable' may not suffice when stability is defined as the permanent coexistence of locally occurring species, because even the composition of the adult gut microbiota appears to change slightly over time, with more taxa shared between shorter sampling intervals relative to long intervals. It seems that the environmental factors that affect the composition mainly exert their effect at the abundance of taxa, but less to their presence., ${ }^{92,}{ }^{43}$. These minor fluctuations in community composition in the absence of large disturbances indicate that long-term stability of human gut microbiota communities is not maintained by inertia, but rather by the action of (unknown) restoring forces within a dynamic system ${ }^{44}$.

Nonetheless, although compositionally the microbiota is very diverse among individuals and can change considerably with respect to relative abundance of its members within one individual in time $\mathrm{e}^{43}$, the corresponding functional profile, based on the collective gene content is quite conserved at the level of COG (Clusters of Orthologous Groups) categories, which is a reflection of the commonly observed high functional redundancy among different bacteria in various habitats, with regards to core functions related to DNA replication and repair $^{45}$. More peripheral functions, such as SCFA production, are also very well conserved ${ }^{46}$. Likely, the human colonic environment invokes similar functionality and functions in individuals. Furthermore, studies have shown that as the number of species per functional group increased, replicate communities were more consistent in biomass and 
density measures and compensation by one species for loss or decline in another preserves long-term average ecosystem performance while variability in performance is reduced. Therefore, functional redundancy and diversity promote the long-term probability of persistence, and enhances resilience to pulse perturbations ensuring the continuity of functions that are important to the host ${ }^{44}$.

In summary, microbiome assembly in newly created habitats such a sterile newborns likely involves a gradual shift from conditions under the strong influence of stochastic and historical factors such as birth mode, medical interventions, and illness, towards conditions increasingly influenced by environmental selection, with weaning as a strong catalyst and with development towards an adult-like composition continuing into childhood. Based on recent, well powered studies the normal/healthy microbiota seems to be dominantly shaped by a history of numerous common environmental exposures from competing sources other than, or in addition to, our family and a much smaller influence of host genotype under normal healthy circumstances. However, in specific cases such as polymorphisms of important mediators of GI immune functioning such as FUT2 and NOD2, which have been associated to an increased risk of distinct GI pathologies such as IBD, the contribution of genetic components might potentially be much higher.

\section{The microbiota and human health}

In the last decade the microbiota has been linked to our health. More specifically, dysbiosis, which refers to perturbations of the 'normal' 'stable' microbiota, has been associated with the development and progression of a plethora of syndromes and physiological aberrations such as, obesity and its associated metabolic disorders (OAMD $)^{47,48}$, autoimmune diseases such as rheumatoid arthritis ${ }^{49}$, type 1 diabetes $^{50}$ and celiac disease ${ }^{51}$, the more obvious functional GI disorders such as $\mathrm{IBS}^{52}$ and $\mathrm{IBD}^{47,53}$, as well as more unlikely conditions such as autism ${ }^{54}$ and Parkinson's disease ${ }^{55}$.

The reasons for such associations are not yet clear and may reflect either causal or secondary processes due to the impact of microbial composition and/or function and the contributions of environment and host genetics. In most cases evidence is based on associations, animal studies (mainly rodents) and in vitro data, but cause consequence data for humans is quite scarce.

\section{Model systems}

There are mainly two different types of model systems to gain more mechanistic insight on many aspects of the microbiota. First, in vitro systems that mimic the conditions of the human GI tract, such as SHIME and TIM-2, have mainly been used to study the effect of dietary and pharmaceutical components on the composition and metabolic output of the microbiota, with regards to genotoxicity, SCFA and other microbial metabolites that might interact with the host. Human in vivo studies are often restricted to faecal samples, which do not provide information on dynamic microbial processes at the site of fermentation in 
the gut. The main advantages of these model systems are that they allow dynamic sampling over time in different consecutive regions of the human colon, and therefore provide insights in different steps of the fermentation process. Each potentially important parameter can be adjusted and there are no ethical constraints for using in vitro models, implying that pathogens and toxic compounds can be used without the need for ethical approval. In vitro approaches thus offer the possibility of deeper mechanistic studies and the development of hypotheses that need to be tested in human clinical trials. In turn, these in vitro culture systems provide an oversimplification of the in vivo situation as only the caecum and proximal colon exhibit characteristics of a continuous culture. They also lack important feedback mechanisms by the host, such as the release of antimicrobial compounds and direct interactions with the immune system ${ }^{56,57}$. These characteristics might be important if a certain composition might be considered dysbiotic only if certain phenotypical host features are expressed. For instance production of toxic metabolites of an IBD patient's microbiota was shown to be higher than that of a healthy individual ${ }^{58}$. However, the physiological conditions for healthy individuals were also used for IBD patients. It is likely that differences may exist between healthy individuals and IBD patients with respect to $\mathrm{pH}$, redox potential, gut transit and dietary habits and thus the substrates that make it to the colon. Therefore, in vitro model systems provide unprecedented mechanistic depth to determine how certain substrates are broken down, by which organism(s) and in which metabolites they might end up. However, in deciphering the role of the microbiota in a host they might paint a picture that is too one-dimensional.

Besides in vitro fermentation models, in vivo animal models, and especially rodent models have been increasingly used in biomedical research. Mice have become the model of choice for most research on the microbiome, because of their low maintenance cost (as compared with other mammalian experimental models), high reproductive rates and short life cycle. However, to interpret and translate the knowledge gained from these studies we need to appreciate and factor in the differences in behaviour and physiology of the GI tract and natural composition of the microbiota between humans and rodents. Overall, the mammalian digestive tract is strongly conserved, thus given their shared omnivorous nature, humans and mice share strong similarities. However, there are some differences in that humans have evolved towards a smaller cecum (with no clear function) and colon and a relatively longer small intestine as compared to rodents. In mice fermentation of indigestible food components is compartmentalized in the cecum while in humans this takes place in the colon ${ }^{59}$. Cells that are essential to intestinal integrity and host-microbiota equilibrium, such as goblet and Paneth cells, are conserved between the two species, although there are differences in distribution ${ }^{60}$. Regarding the composition of the microbiota there is no consensus. Generally it is thought to be relatively similar at higher taxonomic ranks; although it has been shown through the analysis of $16 \mathrm{~S}$ rRNA sequences 
as well as meta-transcriptomes that membership is significantly different at higher resolution $^{61,62}$.

The main advantages of murine models are that they allow extreme interventions and targeting of specific gene/pathways through knock-out models to study the causal role of gut microbiota in health and disease. This combined with the homogenous genetic background of laboratory mice that is achieved through inbreeding and control over sources of variation such as diets and housing conditions limit unwanted influences of noise from the surrounding environment to the gut microbiota and therefore, generate a cleaner system which increases the signal to noise ratio regarding gut-bacteria-host interactions and improve reproducibility of experiments ${ }^{60}$.

Humanized gnotobiotic mice, which result from the inoculation of a human gut microbiota sample in germ-free mice are considered to be the gold standard for confirming associations and trying to prove causality in gut microbiota research ${ }^{22,24,63}$. Globally, they retain a human composition (100\% of phyla, $11 / 12$ classes and $~ 88 \%$ of genus-level taxa), except for the absence of many low-abundance taxa, which might still be relevant ${ }^{64}$. Use of all of these mouse models has brought more insights into the mechanisms and the role of the microbiota in pathologies, such as growth impairment from malnourishment ${ }^{22}$, obesity ${ }^{48,65}$ and $\mathrm{IBD}^{66}$. Nevertheless, it has been shown that they might not fully recapitulate the mechanisms of the human-host-gut-microbiota interaction ${ }^{60}$. It should be highlighted specifically for humanized mice that, although the microbiota reflects the composition of the donors reasonably well, this does not necessarily reflect the real host-microbe relationship observed in humans because the gut microbiota is transplanted into a host with which it has not co-evolved ${ }^{60,67}$.

The advantages of mouse models are also their pitfalls when we want to translate results to human research. All the potential drivers that shape the variability of a 'normal healthy' microbiota in humans ${ }^{68}$, are absent. Biologically there might be considerable advantage in having this diversity in the population, and therefore their microbiota might not reflect the 'real-life' microbiota. All these parameters that help create this relatively strong and clean signal (extreme interventions coupled with low microbial and genetic variability) can pose problems when translating obtained results to humans where the biological signal to noise ratio is likely to be much lower due to more subtle interventions and the dampening effect of homogeneous responses ${ }^{69}$. This said, they are one of very few methods to assess causality in microbiota research, and thus further development and improvement of this approach is essential. For instance the use of wild mice with more heterogeneous genetics and microbiota composition might be needed when we want to translate and confirm the causal relations established in such mouse models. 
The microbiota and disease; functional gastrointestinal disorders as a paradigm

In contrast to in vitro and in vivo model-based studies as described above, studies with human volunteers have largely provided associations. This paragraph is not meant to be exhaustive, and most human pathologies associated with the microbiome have been reviewed in depth elsewhere ${ }^{70}$. This paragraph will use functional disorders of the GI tract (FGIDs) as a paradigm for a majority of human health-microbiota interactions, as they represent the largest and closest host-microbe interface and arguably the most direct effects of any miscommunication would be observed here.

FGIDs typically display a complex multi factorial aetiology and/or are biologically heterogeneous ${ }^{52,71}$. There are several general lines of evidence that suggest a direct involvement of the intestinal microbiota in the pathogenesis of certain FGIDs: gastrointestinal infections and systemic antibiotic use are strong risk factors for their development and in some cases abnormal concentrations of fermentation end products such as SCFAs have been found ${ }^{72}$. In the case of IBS, innate and adaptive immunity involved in sampling and recognition of the microbiota, such as the expression of toll like receptors in the mucosa, antibodies to bacterial flagellin and production of human $\beta$-defensin- 2 have been shown to be different in IBS compared to controls ${ }^{52}$. Differences in the fecal and colonic microbiota have been identified between healthy controls and IBS subjects and among IBS subjects as well. Furthermore, several microbial signatures have been associated with certain demographic or etiological features of IBS. However, a fecal or mucosal microbial signal diagnostic of IBS or of an IBS subtype or subpopulation has yet to be validated.

These inconsistencies may relate to several factors, including methodological differences, such as variations in protocols for sample preparation and analytical approaches, outliers due to small sample sizes and biological differences such as the intrinsic microbiota variability between subjects, the functional redundancy of the microbiota, differences in subject selection, definition of study populations and overlap between the various FGIDs as well as variance in diet, therapy or other environmental exposures which are known to influence the microbiota.

It also needs to be recognized that many studies describe comparisons between different groups of subjects on the basis of a single fecal sample per subject, although the composition of the adult gut composition changes slightly over time, with sometimes considerable changes in the abundance of taxa within one individual. Therefore, such a snapshot of the microbiota in underpowered studies might be very sensitive to outliers and as a result, such comparative analyses cannot differentiate between cause, consequence, or coincidence ${ }^{73}$.

All these factors may, in part, explain why there has been no consensus regarding the association of most FGIDs with a specific microbe or groups of microbes ${ }^{52}$. Therefore, it 
is evident that well powered longitudinal studies involving repeated sampling of the microbiota as well as clinically relevant host characteristics will be crucial during intervention to differentiate cause from consequence or coincidence.

\section{Human interference; antibiotics and resilience}

Now that we are beginning to appreciate the microbiome and its intricate relationship with ourselves, concern is growing about things we are doing that may disturb this system. Antibiotic use is just one example of common (medical) practice that may be altering the human microbiome by reducing, removing, or changing fundamental elements. As mentioned in the beginning of this introduction, infectious diseases have been the leading causes of human morbidity and mortality for most of human existence, and antimicrobials are therefore arguably one of the most successful forms of chemotherapy in the history of medicine. Contrary to common believe, research has revealed the exposure to antibiotics is not confined to the modern antibiotics era, as traces of tetracycline, for example, have been found in human skeletal remains from $35-550 \mathrm{CE}^{74,75}$. However, since penicillin in the 1940s, antibiotics have been in broad use for treating infectious diseases in humans as well as at subtherapeutic levels to stimulate meat production in livestock ${ }^{76}$. However, now that it is clear that we are host to many more microbes with mutualistic rather than parasitic interactions, we have come to realise that besides the targeted organisms there is huge collateral damage with regards to our microbiome. This has been shown for a wide range of sampling strategies, treatment durations, analytical approaches and antibiotics ${ }^{69,} 77-80$. Depending on the antibiotics treatment, the composition as well as the metabolic output of the gut microbiota is altered compared to the routine temporal variability of community composition and function, and most taxa begin to return to prior levels within several weeks. However, various taxa recover to different extents, while some do not recover at all over the duration of the study ${ }^{69,77,79}$. Besides the acute effects, the (past) use of antibiotics, especially in the early developmental phase of the microbiota, has been shown to have lasting consequences regarding composition and metabolism ${ }^{81}$ and has been associated with health complications ${ }^{82}$. However, this is still controversial due to two large confounders in most but not all studies. 1. Reverse causation; where symptoms have caused prescription of antibiotics and 2. Confounding by indication; where (respiratory tract) infections leading to antibiotic use may be the underlying cause triggering symptom development ${ }^{83}$.

Probably the highest risk associated with antibiotics use is the selection and stimulation of antibiotic resistance and the augmentation of antibiotics resistance genes in the surviving gut microbiota ${ }^{84}$. These mobilized resistance genes are a reservoir for drug resistance in potential pathogens, and antimicrobial resistance is currently one of the most important global public health threats that we face $e^{85-87}$. 
Overall, research suggests that generally the human gut microbiota of healthy adults, but not that of very young children and infants, is largely, but not entirely, resilient to short courses of antibiotic therapy. However, there are instances where the microbiota is pushed from its relatively stable state and moves into an unfavourable position through repeated use of antibiotics. The direct effect of a disrupted microbiota is most clearly illustrated in the setting of Clostridium difficile infection.

\section{Microbiota manipulation; the malleable microbiome}

Although it has been exceedingly difficult to produce cause consequence data in humans, a causal relationship between host health and the GI microbiome has been most clearly demonstrated using a radical gut microbiota modulation procedure, which replaces the complete microbiome from a patient with the microbiome from a healthy donor, called a fecal microbiome transplant (FMT). This causality is particularly strong for a very welldefined form of dysbiosis: longstanding recurrent $C$. difficile infections. This can serve as a model for dysbiosis due to its mechanistic simplicity. Here a single opportunistic pathogenic organism (which is a relatively normal member of the human microbiome) proliferates after the microbiota has been extensively disrupted by (repeated) antibiotics use. It then overgrows the microbiota, produces toxins and causes colitis and diarrhoea in patients, which can even lead to death. FMT has been shown to outperform conventional antibiotic treatment, and extremely high success rates of $90 \%$ are routinely reported ${ }^{88}$. These high success rates have seen FMTs used in other clinical settings as well, such as Ulcerative Colitis ${ }^{89}$ and improvement of insulin sensitivity ${ }^{90}$.

Although the microbiota after transplantation resembles the composition of the donor it usually does not end up to be a replica of the donor ${ }^{88,90}$. Intriguingly, the plasticity of the microbiota (the completely different composition of another individual) seems at odds with the fact that the microbiota is highly individual and relatively resilient with regards to its membership, and certain taxa which are thought to be with us over several decades, maybe even coming from the initial birth inoculation'. This observation leads to the question what actually constitutes a normal healthy microbiome and whether there is such a thing. And more fundamentally, how important is the interaction with the host in health as well as disease? This will aid in the definition and understanding of dysbiosis and provide a better understanding of the therapeutic options at our disposal to exploit this flexibility for restoration and modulation of the existing microbiota.

\section{The healthy microbiome}

The last few paragraphs have provided an overview of the current state of the art regarding the factors that shape the microbiome, including the fact that the link with many diseases is often based on correlations and case control studies comparing a certain phenotype with 
healthy individuals (usually individuals that do not exhibit symptoms from the patient group). However, due to the very high inter-individual variation in the human microbiome it has been challenging to define the normal healthy microbiome. Also, the relatively vague definition of health as 'a state of complete physical, mental and social well-being and not merely the absence of disease or infirmity' from the WHO (a definition which has not been amended since 1948) does not help.

Large scale initiatives like the Human Microbiome Project and European Metagenomics of the Human Intestinal Tract (MetaHIT) have generated enormous amounts of sequencing data to expand our knowledge on the structure and function of the normal microbiome ${ }^{6,91}$. However, due the high dimensionality of the data (high inter-individual variability), studies of this type typically require large sample sets for hypothesis testing. Therefore, approaches have been explored that could reduce the dimensionality of this data and simplify it. One such approach is to search for structural patterns, i.e. community compositions that are more prevalent than others, due to certain ecosystem 'rules'. The first effort at communitywide stratification, based on metagenomic data from 39 faecal samples, suggested that the human GI microbiome could be categorized into three types, designated 'enterotypes', which seemed to be independent of phenotypical, genetical or life style characteristics such as age, BMI, health status, gender of demography. These community structures were enriched in either Bacteroides, Prevotella or, in a less pronounced cluster, defined by enrichment of Ruminococcus ${ }^{92}$. However, a later study utilizing the same procedure on metagenomic data from an order of magnitude more individuals (396) failed to clearly discriminate distinct microbiota types ${ }^{7}$. Now it seems the leading hypothesis is that the distribution of the microbiota among individuals resembles more of a gradient with more contrasting abundances of Prevotella and Bacteroides. Another study observed community wide structural patterns in HITChip phylogenetic microarray-derived compositional data of more than 1000 individuals. Lahti and co-workers identified five bacterial groups that displayed robust independent bi-modal abundance distributions, meaning that these taxa were present at either low or high abundance with an unstable intermediate abundance state between the two contrasting states. This instability determined through cross-sectional data was confirmed in a subset of subjects from whom longitudinal data was available. The identified bimodal bacterial groups were Dialister spp., relatives of Bacteroides fragilis, Prevotella melaninogenica and Prevotella oralis (combined as one group) and two groups of Uncultured Clostridiales; UCI and UCII. The abundance state of these groups seemed independent of (short-term) dietary interventions and associated with host characteristics such as BMI and age. For instance UCII showed a very clear shifting state probability from high to low with increasing age. ${ }^{93}$ Interestingly, Prevotella, the most distinct bimodal group, especially regarding the relatively high occurrence of the high abundance stable state was also observed to be a driver of the contrasting states in the 396 metagenomes $^{7,93}$. The 
authors proposed that these 'bacterial dipswitches' could be targeted in the future for therapeutic approaches ${ }^{93}$.

Another avenue that has been explored to simplify this system and to define a normal healthy microbiome is to determine a stable core or possibly a minimal microbiome and answer the question whether a set of key species could perform all the necessary functions of a healthy normal microbiome. Although this question has been extensively studied, there is still no consensus on a phylogenetic (microbiota) core. This is partly due to inconsistencies between the definition of such a core (such as cut-offs for minimum abundance and prevalence of a given phylotype to be considered a member of the core), the phylogenetic resolution at which the core is defined and variations in study populations with regards to variables that are known to influence the microbiota composition, such as age, health status, diet and demography $36,91,94$, ${ }_{95}$ Besides a biological foundation, some of the aforementioned inconsistencies could be appointed to the myriad of technologies that are in use or have been used to study this core and their individual strength and weaknesses, such as the maximum achievable phylogenetic resolution, differences in confidence at low abundance thresholds, the use of different reference databases and the coverage and resolution of different primer pairs ${ }^{91}, 94,96$. However, as mentioned in previous paragraphs, there is a large functional overlap between bacteria, which is not surprising since the common household genes involved in for instance DNA replication and repair are present in all living organisms, and therefore a metagenomic core, instead of a phylogenetic core might be more informative from a biological

\section{Prospectus}

Instead of trying to summarise all the associations and the concomitant bacterial changes, the preceding paragraphs mainly served to provide a biological context and framework for the interpretation of the work described in this thesis.

Human diseases are often multifactorial stochastic processes under the influence of genetics, 'life style' (diet, sports, stress), and environmental factors. In parallel, the adult human GI microbiota is highly complex, very individual, relatively stable with regards to its members (some members more stable than others), yet with large temporal fluctuations in abundance but also relatively resilient against gross perturbations. The genetic variation of the host and high inter-individuality of its microbiota might be biological advantages; however it complicates the life of microbiome researchers. This diversity, in combination with microbe-microbe as well as host-microbe interactions that can largely vary under different circumstances (different disease states), paints a very complex picture.

We know through FMTs that although the microbiome is relatively resilient, it is also changeable. Nevertheless, we do not yet know what a normal healthy composition entails and whether this is universal, i.e. whether the same composition/function is good for 
everyone. By understanding the factors that shape our microbiome under 'normal' conditions and give rise to the observed variation within and between individuals we can study the major factors that shape the microbiome under specific conditions such as certain pathological phenotypes and infer their relative importance under these conditions and develop more focused methodologies to manipulate it under these specific conditions towards a state that is healthy for that specific individual. 


\section{Thesis outline}

Recent evidence indicates that the GI microbiota and its products may contribute to the development of obesity and related diseases, and differences in GI tract microbial ecology between individuals might be an important factor in this complex aetiology. This thesis aims to provide and apply tools to investigate the potential of the gut microbiota as a potential biomarker or therapeutic target and generate leads for mechanisms through which the microbiota mediates these effects.

Chapter 1 serves as reference point regarding our understanding of the structure and function of the normal human GI microbiome and aims to place the observations from the following chapters, namely the deviance of individual taxa or patterns (dysbiosis) from the 'normal' state, into an ecological context. This can be investigated using a diverse set of complementary so called -omics technologies such as 16S rRNA gene-targeted composition profiling, meta-bolomics, -genomics, -transcriptomics and -proteomics. Chapter 2 describes the different molecular approaches and their contributions to our understanding of the role of the GI microbiota in host energy homeostasis. Correspondingly, it highlights their respective strengths, but also tries to create awareness for their specific limitations. Besides the need for intelligent applications of complementary methodologies to answer specific questions regarding the gut microbiome, human gut microbiota research has been hampered by the large heterogeneity in, genotype, lifestyle, diet and the complex aetiology of e.g. OAMD. Therefore, current research should focus on more homogenous subpopulations, through the use of both anthropometric (weight, total body fat) as well as biochemical variables (insulin resistance, hyperlipidaemia) to define better defined categories with less confounding. In Chapter 3 we mined the faecal microbiota from 295 children aged 6-7 years from a cross-sectional cohort, for associations with weight related anthropometric outcomes (age- and sex- standardized weight and BMI $\mathrm{z}$-scores, overweight defined as $\mathrm{BMI} \geq 85$ th percentile). Because of the large amount of available phenotypic data we could extensively control for known confounders such as birth weight, gender and gestational age. Chapter 4 investigated the effects of gut microbiota manipulation by antibiotics ( $7 \mathrm{~d}$ administration of amoxicillin, vancomycin or a placebo) on clinical parameters associated with OAMD, such as tissue-specific insulin sensitivity, energy metabolism, gut permeability and inflammation, in a well phenotyped and relatively homogenous subpopulation of 57 obese yet, pre-diabetic men. Next, in chapter 5 we studied the microbiota of two independent cohorts with comparable strict recruitment strategies (overweight and obese pre-diabetic male subjects), from the Dutch regions of Maastricht (south-east) and Amsterdam (north-west). We searched for associations with biochemical markers for tissue specific insulin sensitivity and weight related anthropometric outcomes. This study is unique as no human data are available that 
investigates whether tissue specific insulin sensitivity, as measured by the golden standard hyperinsulinemic-euglycemic clamp technique, is related to a specific microbial pattern.

The next part of this thesis focuses more on technological aspects associated with sequencing of 16S rRNA gene amplicons, the development of a bioinformatics pipeline for this type of data in chapter 6 and the application to biological data in chapter 7. Although massive high-throughput sequencing of short, hypervariable segments of the 16S ribosomal RNA (rRNA) gene has transformed the methodological landscape describing microbial diversity within and across complex biomes, several studies have shown that methodology rather than the biological variation is responsible for observed sample composition and distribution, compromising true meta-analyses in this research field. Currently, there is a lack of consensus regarding key features of the healthy microbiome, such as richness, diversity and composition, while the link with specific human pathologies often reveals study specific sets of biomarker organisms. Large scale meta-analyses would aid in addressing whether the basis for these differences is biological, technical or maybe a combination of both. To facilitate such metaanalyses of microbiota studies chapter $\mathbf{6}$ describes the development of NG-Tax, a pipeline for $16 \mathrm{~S}$ rRNA gene amplicon sequence analysis and its validation with synthetic communities. In addition, we provide recommendations on an optimal setup and identify critical points in the analysis and interpretation of this kind of complex and very noisy data. Chapter 7 shows an ecosystem approach to assess the temporal dynamics (composition, activity, metabolic output) and reproducibility of all these variables in the TIM2, a dynamic in vitro model of the colon, fed with three different diets: the normal diet and a version of which either the protein or carbohydrate fraction was diluted. We applied the recommendations from chapter 6 to describe the temporal dynamics of microbiota composition and determined the global metabolic function of the microbial communities through metabolomics and metatranscriptomics. Finally, chapter 8 summarizes the research described in this thesis and provides a discussion on its contribution to the current state of knowledge. In addition, it provides a perspective on future research to elucidate the role of the microbiota in OAMD and other human pathologies as well. 


\section{Chapter 2}

Molecular ecological tools to decipher the role of our microbial mass in obesity

Gerben D. A. Hermes, Erwin G. Zoetendal, Hauke Smidt 


\begin{abstract}
After birth, our gastrointestinal (GI) tract is colonized by a highly complex assemblage of microbes, collectively termed the GI microbiota, which develops intimate interactions with our body. Recent evidence indicates that the GI microbiota and its products may contribute to the development of obesity and related diseases. This, coupled with the current worldwide epidemic of obesity, has moved microbiome research into the spotlight of attention. Although the main cause of obesity and its associated metabolic complications is excess caloric intake compared with expenditure, differences in GI tract microbial ecology between individuals might be an important biomarker, mediator or new therapeutic target. This can be investigated using a diverse set of complementary so called -omics technologies such as $16 \mathrm{~S}$ ribosomal RNA (rRNA) gene-targeted composition profiling, meta-bonomics, -genomics, -transcriptomics and -proteomics. This review aims to describe the different molecular approaches and their contributions to our understanding of the role of the GI microbiota in host energy homeostasis. Correspondingly, we highlight their respective strengths, but also try to create awareness for their specific limitations. However, currently it is still unclear which bacterial groups play a role in the development of obesity, in humans. This might partly be explained by the heterogeneity in, genotype, lifestyle, diet and the complex etiology of Obesity and its Associated Metabolic Disorders (OAMD). Nevertheless, recent research on this matter has shown a conceptual shift by focusing on more homogenous subpopulations, through the use of both anthropometric (weight, total body fat) as well as biochemical variables (insulin resistance, hyperlipidaemia) to define categories. Combined with technological advances in the reviewed the molecular techniques, recent data suggests that an OAMD associated microbiota can be characterized by a potential pro-inflammatory composition, with less potential for the production of SCFAs and butyrate in particular.
\end{abstract}




\section{Introduction}

The human gastrointestinal (GI) tract contains several compartments with distinct anatomy and function, and is of utmost importance in supplying the body with energy and essential nutrients by converting and absorbing food components. In total, over 1000 microbial species, which collectively harbour more than 3.3 million unique genes ${ }^{6}$, have been shown to colonize the human GI tract ${ }^{97}$, and are collectively termed the GI microbiome. Recently each individual has been estimated to contain between $101 \pm 27^{9}$ and $>160$ of such species $^{6}$.This vast microbial gene reservoir, which is approximately two orders of magnitude larger than our own, complements human physiology with a range of essential functions, such as biosynthesis of vitamins and breakdown of complex carbohydrates ${ }^{98,99}$. Conversely, an aberrant microbiome has been linked to gastrointestinal disorders ${ }^{52}$.

The microbial ecosystem of the GI tract is very complex because it consists of a wide variety of different species that interact with each other and with the host, and many of the community members cannot be easily grown as pure cultures in a laboratory setting. Nevertheless, the introduction of a broad range of molecular tools over the past two decades has provided unprecedented opportunities to bypass the culturing requirements and study functionality, activity and composition (dynamics) directly.

Application of molecular approaches has revealed that, although the contribution of microbial groups appears to fluctuate in time ${ }^{43}$, the (fecal) microbiome is highly individual and much more stable in adults ${ }^{100}$ and the elderly ${ }^{101}$ than in children. Furthermore, the microbial composition is GI tract location specific ${ }^{34}$ and it seems that host demography (geographic location, diet and genetic background) has a major impact on composition ${ }^{35}$,

102. In addition, it was recently shown that the faecal microbiota of individuals can be largely grouped into three distinct clusters mostly driven by species composition, termed enterotypes $^{92}$. Although the nature of enterotypes (static or continuous), and the number of enterotypes is highly debated, the fact that, despite individual-specific variation, individuals can be classified based on their GI tract microbiota composition is a conceptual breakthrough as this could be important for making associations between microbiota and health aspects. Nevertheless, at least in a western population, the overall functional properties are relatively well conserved, as based on large scale metagenome sequencing, indicating a common structural core ${ }^{6,91}$. Moreover, recent findings suggest that the GI microbiome also plays a role in energy homeostasis of the host ${ }^{48,62,103,104}$. This coupled with the current worldwide epidemic of obesity, has moved microbiome research into the spotlight of attention. Although the main cause of obesity and its associated metabolic complications is excess caloric intake compared with expenditure, differences in GI microbial ecology between humans might be an important, mediator, new therapeutic target, or biomarker to predict metabolic dysfunction/obesity in later life. 
This subject has been approached using different so called -omics technologies such as $16 \mathrm{~S}$ ribosomal RNA (rRNA) gene-targeted composition profiling, as well as metagenomics, metatranscriptomics, metaproteomics and metabolomics, to identify the composition of the microbiota and its overall genetic (i.e. functional) potential and activity, respectively. This review aims to describe these different approaches and what they have contributed to our understanding of the role of the GI microbiome in host energy homeostasis, focusing on Obesity and its Associated Metabolic Disorders (OAMD). Correspondingly, we highlight the strengths of the different approaches, but also try to create awareness for their specific limitations. This serves to illustrate that each method merely discloses a small piece of the microbial puzzle and that their isolated application might paint an incomplete picture. Only truly integrated approaches that combine multiple 'omics' approaches will permit moving from a 'parts list' to a deeper understanding of the functioning of this fascinating and relevant ecosystem ${ }^{34,105,106}$

\section{List of abbreviations}

$\mathrm{B}: \mathrm{F}$ ratio: Bacteroidetes to Firmicutes ratio

BMI: Body Mass Index

SCFA: Short Chain Fatty Acids

COG: Clusters of Orthologous Groups

FISH: Fluorescent In Situ Hybridization

GI: gastro-intestinal

HITChip: Human Intestinal Tract Chip

HGC: High Gene Count

KEGG: Kyoto Encyclopedia of Genes and Genomes

LGC: Low Gene Count

NGS: Next Generation Sequencing

OAMD: Obesity and its Associated Metabolic Disorders

OTU: Operational Taxonomic Unit

PTS: phosphotransferase system

rRNA: ribosomal RNA

T2D: Type-2-Diabetes 


\section{Identifying the players}

It is generally recognized that a major part of GI tract community will remain undetected using classical cultivation approaches. To circumvent this apparent culture bias, the majority of studies employ culture-independent approaches, in which the small subunit or 16S rRNA and its encoding gene are used as a phylogenetic marker to identify, classify and quantify microorganisms that are present.

\section{DNA isolation}

DNA isolation is an integral step in all culture independent, phylogenetic and metagenomic approaches to characterize the highly diverse GI microbiome. Previous studies have shown that differences in cell wall structures cause microbial cell lysis to be more or less efficient ${ }^{107,108}$, which can distort the apparent representation of the composition of microbial communities ${ }^{107-109}$. Currently, mechanical cell disruption by repeated bead beating $(\mathrm{RBB})$ is strongly advised for all applications with the exception of metagenome clone library construction, as $\mathrm{RBB}$ has been shown to produce the highest bacterial diversity and a significantly improved DNA extraction of Archaea from defined pure and mixed cultures, as well as faecal samples ${ }^{108-110}$. The standardization of DNA extraction should be a step towards the comparability and reliable meta-analysis of the results obtained in different laboratories.

\section{Culture independent community profiling}

A variety of useful features render the 16S rRNA gene an ideal target to retrieve both taxonomic and phylogenetic information. Due to its conserved function it is present in all prokaryotes and therefore its sequence has remained relatively conserved throughout evolution. It contains slow-, as well as fast-evolving regions and includes approximately $1500 \mathrm{bp}$, which is sufficient for comparative sequence analysis. Another major advantage of the $16 \mathrm{~S}$ rRNA gene, as opposed to other potential markers, is the availability of large databases such as Greengenes ${ }^{111,112}$, SILVA $^{113}$ and the Ribosomal Database Project ${ }^{114}$, linking 1.400.000 full length sequences to taxonomies. Nonetheless, unambiguous species level resolution using the $16 \mathrm{~S}$ rRNA gene might be unattainable, due to its conserved nature and difficulties associated with an accurate prokaryotic species definition ${ }^{115-118}$.

\section{The Beginnings}

Most culture-independent techniques that have been used for the analysis of the GI microbiome over the past two decades have thus been based on analysis of the 16S rRNA gene and can be roughly divided into two groups. Whole community surveys, that examine the overall diversity in a sample and methods that target a few specific microbial groups. The latter group consists of quantitative (or real-time) PCR (qPCR) and Fluorescent In Situ Hybridization (FISH) coupled to flow cytometry or microscopy for quantification. These technologies are fast, quantitative and with appropriate primer or probe design, highly selective. However, any method that relies on specific oligonucleotide probes is inherently 
limited by the fact that without a 16S rRNA gene based survey of the overall diversity within a sample; the specificity of the selected probes will be uncertain ${ }^{119}$. Furthermore, the GI microbiome is a very complex ecosystem and perturbations are likely to result in, sometimes subtle, whole community changes, rather than alterations in the abundance of the pre-selected groups. This might lead to incorrect conclusions on the effect of an intervention, because either 1) the targeted groups are not affected, which does not mean nothing is happing, or 2) they are affected, but the results lack a proper community context.

In the past tools such as Denaturing or Temperature Gradient Gel Electrophoreses (DGGE, TGGE), have been employed to produce a "fingerprint" of microbial communities based on 16S rRNA gene diversity. By comparing such fingerprints, Zoetendal et al. revealed a remarkable temporal stability and host specificity of the GI microbiome ${ }^{100}$. These methods are based on electrophoretic separation of 16S rRNA gene amplicons on a gel containing a chemical denaturant or temperature gradient, and generate characteristic banding patterns ${ }^{120}$. Application of these fingerprinting techniques is cheap and can be performed relatively fast. However, they also suffer from a number of limitations, such as 1. they lack resolution for a complex community such as the GI microbiome, 2. Quantification is based on band intensity, and 3. Phylogenetic identification is only possible after excising bands for sequencing or matching migration patterns with those of clone libraries. In turn, Sanger sequencing of $16 \mathrm{~S}$ rRNA clone libraries has expanded our understanding of the microbial composition of our GI tract. Improving mainly on identification potential, it showed that two microbial phyla, the Firmicutes and the Bacteroidetes, are mostly represented in clone libraries representing our GI microbiome ${ }^{121}$. Although, the analysis of complete 16S rRNA gene sequences has advantages for applications such as classification ${ }^{122}$ or defining novel $\operatorname{taxa}^{123}$ the cost per sequence is high and consequently it provides an incomplete view of the microbial composition, revealing only the most abundant taxa, and furthermore generally does not allow analyzing multiple samples at appropriate spatio-temporal resolution. Hence, fingerprinting methods such as DGGE, and Sanger sequencing of clone libraries, are complementary methods. Fingerprinting provides a much clearer picture of the overall diversity and its dynamics in time and space, whereas cloning and sequencing enables identification of specific targets.

Nevertheless, these tools have now largely been replaced by phylogenetic microarrays and Next Generation Sequencing (NGS) of amplicons. These can interrogate community structures at much higher resolution by revealing taxa that are less abundant at much reduced cost, providing the necessary means to compare the microbial composition in a large number of samples simultaneously, and thus allowing to discern spatio-temporal patterns of microbial community dynamics. 


\section{A new century - High-throughput high-content molecular tools}

Next generation sequencing approaches

Next generation sequencing technologies, and in the beginning especially pyrosequencing (as implemented in the Roche 454 Genome Sequencer GS, FLX and FLX Titanium), have revolutionized 16S rRNA based community profiling of the GI microbiome. Pyrosequencing generates much more reads at much lower cost per nucleotide, albeit initially of much shorter length compared to traditional Sanger sequencing. Nevertheless in 5 years the read length increased from $<250$ bases using the GS platform, to $>500$ bases with the Titanium chemistry. Moreover, the advent of barcode identification tags ${ }^{124}$ enabled researchers to analyze hundreds of samples in multiplex at higher depth and lower cost, compared to Sanger sequencing ${ }^{36,77,125}$. Recent advances in read length for Illumina's sequencing by synthesis platforms (Genome Analyzer IIx, Hiseq2000 and Miseq) offered another sharp decrease in sequencing cost, by generating 10-1000 fold more reads at a fraction of the cost per megabase, compared to pyrosequencing ${ }^{126}$. Nonetheless the read length was back to that of the starting days of pyrosequencing at $2 \times 75-2 \times 150 \mathrm{nt}$ and most recently $2 \times 250-2 \times 300 \mathrm{nt}$ for MiSeq, and novel strategies had to be developed to cope with the decreased taxonomic resolution and increased computational demands ${ }^{127-130}$. This has led to sequencing of samples at an unprecedented depth of millions of reads per sample ${ }^{128}$. However the true strength might lie in the opportunity to examine thousands of samples simultaneously at sufficient depth, as was demonstrated by Caporaso et al. who analyzed almost 2000 samples to show the variability of the human microbiome over time in two subjects $^{43}$. Similarly, Yatsunenko et al. studied the fecal microbial communities of more than 500 Malawians, Amerindians and US children and adults, at an average depth of $>106$ reads per sample to reveal profound differences in bacterial assemblages between US residents and those in the other two countries at different ages ${ }^{35}$.

So, how does it work?

Getting the most taxonomic information from next generation amplicon sequencing requires careful selection of sequencing technology, 16S rRNA gene region and corresponding primers, in order to obtain the most useful data, as sequencing platforms not only differ in read length output, but also error rates, error profiles and technical restrictions such as maximum amplicon size ${ }^{126}$. Illumina-based approaches provide the option of sequencing amplicons in both directions. This allows for different strategies; the reads can be made to overlap or the researcher is left with a gap in the middle of the amplicon. When using non overlapping reads, the $3^{\prime}$ and 5 ' reads can be analyzed separately ${ }^{128}$, or joined prior to Operational Taxonomic Units (OTU) clustering and aligned to a trimmed reference database, which improves the phylogenetic resolution by doubling read length ${ }^{130}$. Likewise, if the selected $16 \mathrm{~S}$ rRNA window is small enough for the reads to overlap, a consensus sequence for the overlapping region can be established, effectively increasing the overall 
quality score and taxonomic classification confidence of the resulting constructs ${ }^{131,}{ }^{132}$. After amplification and sequencing, the data can be analyzed using a number of established pipelines, such as QIIME $^{133}$ or MOTHUR ${ }^{134}$. The reads are de-multiplexed and quality filtered, since both sequencing and PCR errors have been shown to lead to overestimation of diversity within samples (alpha diversity) ${ }^{135-138}$. The reads are then clustered de novo into groups of similar sequences, called OTUs, which are often regarded as a synthetic proxy for microbial species as they are typically clustered at $97 \%$ sequence similarity (This threshold was chosen because isolates with an overall genome relatedness of $>70 \%$ based on genome DNA-DNA hybridization was consistent with recognized phenotype-based species classifications at the time ${ }^{139}$ ). Lastly taxonomies can be inferred by associating OTU's to reference sequences in $16 \mathrm{~S}$ rRNA databases using different approaches ${ }^{12-114}$. However, recently, Sun et al. benchmarked several currently used OTU clustering and taxonomy assignment algorithms and found that existing methods can yield vastly different results, marking that the shortcomings of many existing methods have not yet been fully recognized ${ }^{140}$. Alternative to de novo OTU clustering, for very large datasets, such as those produced by the Illumina sequencing platforms, a reference database pre-clustered at $97 \%$ can be used as seeding database for OTU clustering. This approach has the advantage of acting as an additional quality filter because denoising algorithms for Illumina data are still unavailable, given that non-aligning sequences are discarded ${ }^{35}$, and enables studies that target a different variable region to be compared when they are aligned to the same database $^{43}$. It should be kept in mind, however, that different regions are usually amplified utilizing other primers that can introduce their own distinctive biases. Moreover, preclustered seed databases need to contain a good representation of the community of interest, as new species discovery is not possible.

The currently available high resolution, high throughput molecular profiling technologies, sacrifice phylogenetic resolution for a better overall picture, compared to traditional Sanger sequencing of clone libraries. They can only utilize a smaller fragment of the 16S rRNA gene, which potentially reduces the amount of phylogenetic information that can be recovered. Moreover, the different hypervariable regions differ in their taxonomic resolution, and therefore, the target needs to be carefully chosen to account for the extent of phylogenetic information that can be generated by the fragment. One way to analyse the taxonomic usefulness of different hypervariable regions is to compare the taxonomic assignments of a short region to that of the full sequence ${ }^{125,141,142,143,144,145}$. This information and the distinctive structure of the 16S rRNA gene with its hypervariable regions and alternate conserved sequence domains, has allowed researchers to design broad spectrum PCR primers targeting specific regions. Nevertheless, in silico generated primer pairs still need careful experimental validation, as unanticipated biases in the PCR reaction can create deviant profiles ${ }^{127}$. Currently, there is a large choice of PCR primers targeting the $16 \mathrm{~S}$ rRNA gene, and primers should be carefully selected based on 1 . The compatibility of 
the resulting fragment length with the sequencing platform, 2 . The degree of specificity for amplifying microbial sequences compared to host sequences, 3 . The taxonomic coverage desired and 4. The complexity or diversity, of the community of interest. For example, the popular primer pair 27F-338R, which is specific for bacteria (as opposed to eukaryotes and Archaea) was shown to poorly amplify the $16 \mathrm{~S}$ rRNA gene of members of the genus Bifidobacterium, which is a very important member of the human GI tract microbiome ${ }^{146}$. Likewise, due to primer bias, the presence of the phylum Verrucomicrobia was thought to be minimal in soil samples, but was found to be present at relative abundances as high as $20 \%{ }^{147}$.

Overall, the human GI microbiome has been interrogated, targeting several hypervariable regions of the 16S rRNA gene either separately or in tandem (Table 1) and, mainly driven by continuing technological improvements in both sequencing technology and bioinformatics, new primers are being designed ${ }^{148}$. Although several studies comparing the classification suitability of several regions have concluded that different regions produce different representations of the community, it remains unclear if one region is fundamentally superior ${ }^{91,127,}{ }^{149-151}$, even though the application of V6-V9 is generally cautioned against ${ }^{149,151}$. Hence there seems to be no consensus on what seems to be the "best" region, and the use of multiple regions may, collectively, give the most complete description of a community ${ }^{150}$.

Because of the differences in applied sequencing technology and, more importantly, variation in methods used for DNA isolation and choice of targeted variable regions and primers, true meta-analysis has proven virtually impossible, and researchers need to be careful, when interpreting and comparing results obtained by targeting different variable regions using different sequencing technologies.

\section{Validation}

An important distinction between whole genome sequencing, which has driven the development of many sequencing methods, and microbiome profiling by 16S rRNA gene sequencing is that in whole genome sequencing, the error rates are less important as each region of the genome is sequenced many times. By contrast, in 16S rRNA gene amplicon sequencing, each fragment may be sequenced only once. Thus, the effect of raw sequencing error rates on the observed microbial diversity is potentially great, as every erroneous read could be portrayed as arriving from a novel organism ${ }^{126}$. This effect is now typically evaluated on the basis of a synthetic, or 'mock', community that is created by pooling genomic DNA or cloned 16S rRNA gene fragments of multiple isolates ${ }^{128,151,152}$. Ideally, near-full-length $16 \mathrm{~S}$ rRNA gene sequences are available at reference quality for the organisms in the mock community; this allows a description of the effect of raw sequencing error rates on the number of operational taxonomic units (OTUs) and a calculation of the actual error rates and error types ${ }^{153}$. In addition to the assessment of errors, the mock 
community can be utilized in $16 \mathrm{~S}$ rRNA window selection, to determine which variable regions most accurately describes its composition ${ }^{151}$. Most recently, a method was developed, termed Low Error Amplicon Sequencing (LEA-Seq) that provides multiple coverage also for single amplicon sequencing. By combining initial linear PCR to generate individually barcoded amplicons with subsequent exponential PCR an approximately 20fold coverage of all amplicons was reached, allowing the generation of robust consensus sequences ${ }^{9}$. By sequencing mock communities, LEA-Seq was tested against other existing 16S rRNA gene sequencing methods. Relative to the existing standard approaches, LEASeq produced amplicon sequences with higher precision at lower abundance thresholds (a cut-off for how many times a read has to be present as percentage of the whole dataset to be considered valid). At a threshold of $\geq 0.01 \%$ of the reads, LEA-Seq enabled a precision $([$ precision $=($ true positives $) /$ (true positives + false positives) $]$, with a true positive being defined as being $100 \%$ identical across $100 \%$ of the length of the $16 \mathrm{~S}$ rRNA gene sequence of the reference genome) of 0.83 and 0.63 for the V4 and V1-V2 region, respectively, compared to 0.08 and 0.09 for Illumina Miseq and 454 pyrosequencing for the same region. This enabled the authors to determine the stability of the human GI microbiome of 37 healthy individuals with higher confidence and higher depth than previously possible with sequencing.

Table 1. Combinations of sequencing technologies and 16S rRNA gene based variable regions, targeted for human GIT microbiota profiling. This table is not meant to be exhaustive, but serves to illustrate the variety of methods and regions used for GI microbiota profiling, based on sequencing of $16 \mathrm{~S}$ rRNA amplicons.

\begin{tabular}{|c|c|c|c|c|}
\hline & & Technology & & \\
\hline \multirow[t]{2}{*}{ Region } & Sanger sequencing & Pyrosequencing & & Sequencing by synthesis \\
\hline & ABI 3730 & 454 GS FLX & 454 GS FLX & Illumina Illumina \\
\hline $\mathbf{V} 2$ & & 36 & & \\
\hline V3 & & 77 & & \\
\hline V4 & & $102,154,155$ & 102 & $43 * *$ \\
\hline V6 & & $36,77,154,155$ & & \\
\hline V1-V2 & & & 91,156 & $127 * \#$ \\
\hline V2-V3 & & & & $127 * \#$ \\
\hline V3-V4 & & & & $127 * \#$ \\
\hline V4-V5 & & & & $127 * \#$ \\
\hline V5-V6 & & & & $127 * \#$ \\
\hline V6-V7 & & & & $127 * \#$ \\
\hline V7-V8 & & & & $127 * \#$ \\
\hline V8-V9 & & & & $127 * \#$ \\
\hline V1-V3 & & & $149-151,157$ & \\
\hline V3-V5 & & & $91,150,151$ & \\
\hline V6-V9 & & & 151 & \\
\hline full length & $36,77,104,121,151$ & & & \\
\hline
\end{tabular}




\section{Phylogenetic Microarrays}

Another class of tools that allow for high resolution interrogation of the GI microbiome are phylogenetic microarrays, such as the High Taxonomic level Fingerprint of the human intestinal Microbiota Array (HTF-Microbi.Array) ${ }^{158}$, Human Intestinal Tract Chip (HITChip) ${ }^{159}$, PhyloChip ${ }^{160}$ and Microbiota Array ${ }^{161}$. They are basically glass surfaces, each the size of a microscope slide, that are spotted with thousands of covalently linked DNA probes, complementary to $16 \mathrm{~S}$ rRNA gene sequences, that can be hybridised with DNA or RNA. For example, the (HITChip), a 16S rRNA gene tiling array developed at the Laboratory of Microbiology at Wageningen University, consists of 3,600 18-30nt oligonucleotide probes that overlap in sets of three, targeting the V1 and V6 region from 1,033 phylotypes selected from more than $16,00016 \mathrm{~S}$ rRNA gene sequences identified in the human GI tract using $98 \%$ sequence similarity as threshold for phylotype definition ${ }^{159}$. These microarrays are hybridized with fragmented full length $16 \mathrm{~S}$ rRNA, in vitro transcribed from PCR amplicons. Using the HITChip for comparing phylogenetic profiles of fecal microbiomes from five young and five elderly adults collected at three time points, Rajilić-Stojanović and colleagues confirmed previous findings that the adult fecal microbiome is highly individual-specific and relatively stable over time ${ }^{159}$. More recently, the HITChip was used to profile the GI microbiome a range of different populations of healthy subjects and of patients suffering specific intestinal or more systemic diseases, including a group of patients with recurrent $C$. difficile infections who had received an duodenal infusion of healthy donor feces as an alternative to antibiotic treatment, and showed a significantly higher resolution of $C$. difficile-associated diarrhea compared to the antibiotic treatment group ${ }^{88}$. After donor-feces infusion, patients displayed an increased fecal bacterial diversity, similar to that in healthy donors, with an increase in members of the Bacteroidetes phylum, Clostridium clusters IV and XIVa and a decrease in Proteobacteria.

The main advantages of phylogenetic microarrays as compared with other methodologies include (1) ability to profile one sample at a time, which is useful in clinical studies and as a diagnostic tool; (2) the quantitative nature of the acquired data allowing direct comparison of relative abundance levels of each OTU between samples with very high reproducibility; (3) short processing and data acquisition times. However, HITChip analysis comes at higher or comparable costs compared with next generation sequencing, depending on the sequencing platform utilized and the resolution required, which is estimated to be the equivalent of 200.000 250nt FLX pyrosequencing reads per sample (Figure 1) ${ }^{155}$. The HITChip has been benchmarked to pyrosequencing and showed high concordance for fecal samples, whereas small intestinal samples showed lower agreement ${ }^{155,156}$. This brings us to the main limitation of microarrays; their inability to reveal novel species in any sample, because the arrays can only detect those sequences for which they contain probes, although there are probes with higher level specificity, which enable the detection of OTUs that were 
not targeted initially in the design. In addition, the design, use, and analysis of microarrays is technically demanding and requires extensive testing, validation, and optimization ${ }^{162}$, although it should be noted that this is essentially the same for NGS approaches. In turn, this extensive validation and high sample compatibility has allowed for a meta-analysis of 1000 phylogenetic microarray datasets ${ }^{163}$ and a follow up study including $>3000$ samples is currently underway.

\section{S rRNA gene amplicon based approaches}

\section{Next Generation sequencing}
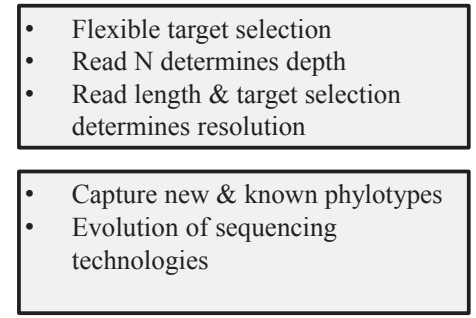

\begin{tabular}{|l|}
\hline Every change in analysis requires \\
careful validation \\
Unfeasible meta-analysis (due to \\
flexibility of target, sequencing \\
platform and pipeline - selection)
\end{tabular}

* 2x 100.000 GS FLX reads ${ }^{155}$

\section{Microarrays}

Fixed target (HITCHip: V1-V6; minimal redundancy) Ultra deep* and quantitative

Very high reproducibility - Allows for meta-analysis

- Captures only known \& related phylotypes

Comprehensive design and validation requirements

Figure. 1. Comparison of two $16 \mathrm{~S}$ rRNA gene amplicon based approaches: microarrays and next- generation sequencing.

\section{GI microbial composition and obesity}

While initial germ free mice studies that suggested a role for the GI microbiome in host energy metabolism were performed from a host perspective ${ }^{103}$, a phylum level compositional shift was associated with the microbiome of obese animals ${ }^{62}$. This increase in the relative abundance of Firmicutes and a proportional decrease in the Bacteroidetes was confirmed in a human dietary intervention study, that showed that weight loss of obese individuals (BMI>30) was accompanied by an increase in the relative abundance of Bacteroidetes ${ }^{104}$. Further research, involving genetically obese conventional and humanized gnotobiotic mice and a diet-induced obesity following a "typical" western style diet high in fat and sugar, corroborated the earlier findings regarding a decrease in relative abundance of members of the Bacteroidetes phylum in the GI tract of obese animals. Moreover, GI microbial transplants from obese to germ free animals led to additional weight gain compared to mice that had received at "lean" microbiome, despite similar food intake. This fuelled the hypothesis that the obese microbiome possesses an increased capacity for energy harvest through a higher production of SCFAs, which in combination with their 
signalling capacity to the host, can contribute to the development of an obese phenotype $\mathrm{e}^{48}$, 164. This body of work demonstrated the potential relationship between GI microbiome (composition) and host energy harvest and fat storage.

Diet induced obesity in mice

Although the hallmark studies of the Gordon lab demonstrated an association between obesity and GI tract microbiome composition, Hildebrandt et al. reported a high fat diet to cause large alterations in GI microbial composition independent of obesity, which included the concomitant decrease in Bacteroidetes and increase of Firmicutes but also Proteobacteria ${ }^{165}$. This study used RELM $\beta$ _knockout mice, which remain comparatively lean when fed a high fat diet, compared to wild type mice. This was observed both for the obese and lean genotypes (i.e., in the presence and absence of genes associated to obesity), indicating that mainly the high-fat diet itself, and not the obese state, accounted for the observed changes in the gut microbiome. Even more striking are the observations by de Wit et al., who employed a phylogenetic microarray and reported a similarly high Firmicutes to Bacteroidetes ratio in the distal intestine of mice fed a high fat diet, but only in animals that consumed a diet high in saturated fat, as opposed to mono- or polyunsaturated fat. The primary trigger for these observed compositional differences was likely an overflow of dietary fat to distal parts of the intestine ${ }^{166}$. Another study confirmed the link between high fat feeding, rather than genetically induced obesity, and this "obesity associated" microbial composition in the murine GI tract. Additionally, changes in the proportions of the major phyla were unrelated to markers of energy harvest (fecal acetate and energy content). Furthermore, these markers changed over time, which led the authors to conclude that, due to the possibility of microbial adaptation to diet, time should be an important consideration in future studies ${ }^{167}$. This also highlights a major shortcoming of the transplantation studies that showed increased weight gain and fat mass in mice that received an obesity associated microbiome, as these interventions only lasted for 2 weeks $^{64}$. Nevertheless, the possibility that changes in GI microbiome composition, primarily induced by diet, can subsequently contribute to development and/or progression of metabolic disorders cannot be excluded. Also, recent evidence has come to light that both diet composition and host adiposity impact microbiome composition, possibly through leptin (a satiety hormone, associated with loss of body fat) mediated with regulation of mucus production and/or inflammatory processes that alter the gut habitat ${ }^{168}$.

Microbiome composition and obesity and its associated metabolic disorders, in humans In contrast to rodent models, human studies employing different methodologies (FISH, qPCR and sequencing) have largely shown conflicting results. The obesity-associated decrease in the ratio of Bacteroidetes to Firmicutes (B:F) proposed by Ley et al. has been extensively reviewed elsewhere ${ }^{119}$, and it is still controversial as it has been both confirmed $^{36,104,169}$ and refuted ${ }^{170-172}$. Schwiertz et al. even reported the opposite trend, i.e. 
an obesity-associated increase in the B:F ratio ${ }^{173}$. More recently, two studies employing NGS of faecal samples from hundreds of individuals also did not find a significant correlation between $\mathrm{BMI}$ and $\mathrm{B}: \mathrm{F}$ ratio ${ }^{92,}{ }^{157}$. Interestingly, Verdam et al. reported a negative correlation between BMI and B:F ratio, using the HITChip, but they found that this was particularly characteristic for severely obese individuals with a BMI $>35^{174}$. Alongside obvious differences in utilized technologies, this inconsistency may partly be explained by the heterogeneity among human subjects, with respect to their genotype and lifestyle as well as the specificity of an individual's microbiome. Furthermore, the microbiome is exposed to fundamentally different 'environmental' factors in both an obese and lean state that go beyond BMI alone, such as the substantial impact of the $\operatorname{diet}^{102,175}$ and host hormonal factors. Moreover, the etiology of obesity and its metabolic complications, including hyperlipidemia, hypertension, low grade inflammation, glucose intolerance and diabetes reflect the complex interactions of these multiple genetic, behavioural, and environmental factors ${ }^{157}$. Lastly, the accuracy of BMI as an indicator for obesity is actually quite limited ${ }^{176}$ and $25 \%$ of obese people are in fact regarded as metabolically "healthy", i.e. they have normal lipid and glucose metabolism ${ }^{177}$.

Linking GI tract composition directly and exclusively to obesity in humans appears to be challenging due to a variety of confounding factors that exist within the heterogeneous human population. This has led to a conceptual shift from treating obesity as a single phenotype, to the correlation of microbial signatures to distinct or multiple features associated with the (development of) metabolic syndrome. Furet et al. employed qPCR to study the microbiome of morbidly obese individuals who had undergone gastric bypass surgery, compared to lean controls. They reported the Bacteroides/Prevotella group to be associated with obesity, but this correlation was highly dependent on caloric intake ${ }^{178}$. Zhang et al. studied the effects of the same surgical procedure using a sequencing approach and reported a significant decrease of Firmicutes and proportional increase of GammaProteobacteria in the post-gastric-bypass individuals that may reflect the combined impact of the gut alteration caused by the surgical procedure and the consequent changes in food ingestion and digestion ${ }^{171}$. Duncan et al. showed a diet dependent decrease of a group of bifidobacteria and butyrate producing Firmicutes in obese individuals on a low carbohydrate weight loss diet ${ }^{170}$. Moreover, Jumpertz et al. investigated the role of the microbiome as a regulator of nutrient absorption in humans. They tested how GI bacterial community structure is affected by altering the nutrient load in lean and obese individuals and whether their microbiome composition was correlated with the efficiency of dietary energy harvest ${ }^{179}$. They found that the alteration of the nutrient load induced rapid changes in GI microbiome composition, but more interestingly, they could not detect differences in bacterial abundance between the lean and obese groups at baseline, which could be because stool samples were collected after subjects had been on a weight maintaining diet. Their results on relative over- or underfeeding were also consistent with the association of a 
calorically more-dense, high-fat/high-sugar diet with an increase in the relative abundance of Firmicutes and a decrease in Bacteroidetes. They attributed the relative changes in the proportional representation of the major phyla to a higher caloric load; however, they could not determine whether it was due to the increased total fat in the diet. Taken together, these observations suggest that diet plays an important role in modulating the GI microbiome and indicate that dietary variations must be taken into account in human studies ${ }^{179,180}$.

Munukka et al. compared the microbiome of overweight women with and without metabolic disorders using FISH and found members of the Eubacterium/Clostridium coccoides group to be proportionally higher in the group with metabolic disorders but not in the non-metabolic disorder and normal weight group ${ }^{181}$. Furthermore, Larsen et al. reported that the B:F ratio was positively correlated with plasma glucose concentration, yet not with $\mathrm{BMI}^{182}$. The role of GI microbial composition and diabetes type 2 has been reviewed by Greiner and co-authors ${ }^{183}$. Until recently no causal relationship had been established between host glucose homeostasis and GI microbial composition, yet Vrieze et al. transplanted the faecal microbiome from lean donors to individuals with metabolic syndrome and increased their insulin sensitivity significantly ${ }^{90}$. The faecal microbiome of obese subjects was characterized by lower microbial diversity, higher amounts of Bacteroidetes, and decreased amounts of Clostridium cluster XIVa as compared with lean healthy subjects. Allogenic transplant (from two different individuals) showed an increase in microbial diversity, as well as an increase in faecal butyrate concentrations and the relative abundance of bacteria related to the butyrate producing Roseburia intestinalis.

\section{Heterogeneity in human populations}

Recently, researchers have also attempted to reduce the potentially confounding influences of human heterogeneity, by studying twins $\mathrm{s}^{36,184,185}$, which have a more similar microbiome composition than unrelated subjects ${ }^{186}$, or by selecting subjects belonging to a culturally and genetically homogenous population ${ }^{157}$. In an attempt to exclude the influence of genotypic differences, Tims et al. compared twins discordant for BMI using the HITChip ${ }^{184}$. They reported a positive correlation of the higher BMI twins with the relative abundance of relatives of Eubacterium ventriosum and Roseburia intestinalis and an inverse correlation with relatives of Oscillospira guillermondii. When co-occurrence networks were built, the former two appeared in a network of butyrate producers and the latter in a network of primary fiber degraders, implicating a role for increased energy extraction through SCFA production. However, the potential impact of the diet on these findings was not determined (for instance a higher plant polysaccharide consumption in the lower BMI groups, being the basis for a higher abundance of primary fiber degraders). ${ }^{184}$. Zupancic et al. used a sequencing approach to study subjects that belong to the old order Amish sect, which exhibits great uniformity of socioeconomic status and lifestyle and less genetic heterogeneity compared with the general population. Whereas no correlation was 
observed of obesity and metabolic syndrome traits with B:F ratio, network analysis revealed 22 bacterial species and 4 OTUs that were significantly correlated with BMI and several features of the metabolic syndrome. In line with the observations by ${ }^{184}$ the relative abundance of Oscillospira guillermondii was inversely correlated with metabolic syndrome traits. It also played role as a central node in one of the identified microbial networks. While the majority of correlations were observed between single metabolic traits and taxa, Lachnobacterium bovis and Anaerotruncus colihominis were inversely correlated with both high BMI and elevated serum triglycerides. Both species are known to produce short chain fatty acids as end-products of metabolism ${ }^{157}$, even though it should be noted that this is the case for many bacteria that are known to inhabit the human GI tract.

Together, these studies show that currently it is still unclear which bacterial groups play a role in the development of obesity in humans, which might partly be explained by the heterogeneity in genotype, lifestyle, diet and the complex etiology of obesity and associated metabolic disorders. Several studies have revealed the diet to be a major factor influencing GI microbial composition, with nutrient load and dietary fat being strong contributors towards an increase in Firmicutes and proportional decrease in Bacteroidetes. Furthermore this decreased B:F ratio seems to be observed particularly for severely overweight individuals. Nevertheless, bacteria thought to be involved in SCFA, and mainly butyrate, production keep emerging differential in most correlative studies.

The subject of a gut microbial composition signature associated with obesity remains controversial, not only because of a number of confounding factors, but also due to the observation that it is still unclear how variation in the composition of species in the microbiome affects the metabolic activity of the community and consequently the host, as we are still hindered by our limited ability to infer organismal function from 16S rRNA gene sequences. Nonetheless, recently a computational approach to predict the functional composition of a metagenome using marker gene data and a database of reference genomes has been developed. PICRUSt (phylogenetic investigation of communities by reconstruction of unobserved states) uses an ancestral-state reconstruction algorithm to predict which gene families are present and then combines gene families to estimate the composite metagenome ${ }^{187}$. It has been validated by recapturing key findings from the Human Microbiome Project ${ }^{91}$ by accurately predicting the abundance of gene families, by using $16 \mathrm{~S}$ rRNA gene information. Their results demonstrate that phylogeny and function are sufficiently linked that this 'predictive metagenomic' approach could provide useful insights in the absence of comprehensive metagenome sequencing. So, 16S rRNA based approaches are especially useful for describing the microbial diversity in the human GI tract to find the potential links between microbes and a certain human health status, and recently also for inferring a synthetic metagenome. But to comprehensively determine its metabolic output and thus the potential impact on host metabolism requires the help of molecular 
tools that reveal the function of the GI microbiome, including metagenomics and other functional omics tools, which will be discussed below.

\section{Defining the genetic potential of the community: metagenomics}

Metagenomics is defined as the study of collective genomes from an ecosystem that can be used to study the phylogenetic, physiological and functional properties of microbial communities $^{188}$. Initial studies involved the generation of large-insert clone-libraries that can be used for sequencing of clones of interest as well as function-driven analysis ${ }^{188}$. Sequence-driven analyses are basically performed to obtain a snapshot of the genetic diversity of an ecosystem, while function-driven analyses are done to screen the library for novel enzymes, such as carbohydrate active enzymes, which are of particular interest in GI tract research since SCFA production through carbohydrate fermentation is a well know way of the microbiome to supply the host with additional calories ${ }^{189}$. Tyson et al. demonstrated that random shotgun sequencing of a small insert plasmid library prepared from environmental DNA can be used to reconstruct a simple microbial community, providing a model for the analysis of other communities that are more complex ${ }^{190}$. Using this approach Gill et al. started to define the gene content and encoded functional attributes of the fecal microbiome of two healthy human adults ${ }^{191}$. A year later, Kurokawa et al. published a comparative metagenomics study that involved 13 fecal samples from adults and unweaned infants ${ }^{192}$. This led to the realization that in fact humans can be viewed as a super-organism whose own metabolism is complemented by microbial attributes. Shotgun sequencing from metagenomic clone libraries has been largely replaced by cheaper, highthroughput shotgun sequencing on next generation technology machines, generally producing short reads. This strategy was employed by Qin et al., who in 2010 mapped the human gastrointestinal metagenome, establishing a human gut microbial gene catalogue that contained 3.3 million non-redundant genes and defined and described the minimal gut metagenome (genes involved in the homeostasis of the whole ecosystem, encoded across many species) and the minimal gut bacterial genome (the functions necessary for a bacterium to thrive in a gut context) ${ }^{6}$. At a sequencing depth of on average $4.5 \mathrm{~Gb}$ per sample, they found that almost $40 \%$ of the genes from each individual are shared with at least half of the individuals of the cohort, which comprised of 124 European subjects. The size of this core exceeded by several-fold that of the core metagenome reported previously ${ }^{36}$. However, using NGS to increase the amount of data to billions of bases per sample still shows limitations in generating high-quality de novo assemblies, as Qin et al. only managed to assemble $42.7 \%$ of the short reads into 6.58 million contigs of $>500 \mathrm{bp}$. The challenge and usefulness of metagenomic assembly is unlikely to be solely addressed by increasing the depth of coverage, and improvements will depend on both the nature and the quality of the sequence data used as input, as well as the quantity and quality of sequenced reference genomes. At the time Qin et al. were only able to align $48.8 \%$ of the 
reads to 194 publically available human gut bacterial genomes. However, this number is increasing rapidly with a reported 178 sequenced genomes in $2010^{193}$ to 800 in $2012^{91}$, which should allow for a more comprehensive view of gut microbial community functional capacity.

\section{Metagenomics and obesity and its associated metabolic disorders}

Turnbaugh et al. combined 16S rRNA based profiling and a metagenomics approach on a small subset of samples, to study the human microbiome in twins concordant for leanness or obesity. Despite discovering a very high level of functional similarity, (pairwise comparisons of metabolic profiles revealed an average $R^{2}$ value of 0.97 ), they identified non-core associated functions that were enriched or depleted in the microbiome of obese subjects $^{36}$. Furthermore, taxonomic classification of metagenome reads confirmed the observed 16S rRNA gene-based compositional differences with respect to lower relative abundance of Bacteroidetes and higher relative abundance of Firmicutes and Actinobacteria in obese subjects. For example, the obese human gut microbiome was enriched for genes encoding phosphotransferase systems (PTS) involved in microbial processing of carbohydrates, pointing again in the direction of increased energy harvest.

Shotgun sequencing of community DNA may capture functional differences in metabolic potential, yet comparative metagenomic analysis of the human microbiome has revealed high functional uniformity across samples ${ }^{36,91}$ and only identified a small set of microbial genes or pathways that appear to be associated with host obesity ${ }^{36,106}$. However, Greenblum et al. employed a metagenomic systems biology approach, generating community level metabolic networks from the fecal microbiome of 124 unrelated individuals and 6 monozygotic twin pairs ${ }^{194}$. By placing variations in gene abundance in the context of these networks, they showed that genes associated with an obese phenotype tended to be located on the outside edge of these metabolic networks suggesting that they encode metabolic steps that are relatively remote from the core of the network. The most remote enzymes represent either the microbiome's first metabolic steps (i.e. its substrate is not produced by any another enzyme in the microbiome) or the last (i.e. enzymes that produce metabolites that are not utilized by any other enzyme in the microbiome). These enzymes are most likely to use or produce metabolites that characterize the gut environment, representing an interface between host and microbe metabolism. Among the differentially identified genes were again those encoding PTSs, but also genes involved in xenobiotic metabolism (choline and p-cresol) and the production of $\mathrm{N}_{2}$. The authors further demonstrated that obese microbiomes are less modular (a specific network-level feature) than those of healthy adults, typically seen as an adaptation to low-diversity environments (i.e. an environment with lower microbial diversity as reported by ${ }^{36}$. Such a systems biology approach gives the opportunity to study higher order modes of deviation from a normal microbiome. Qin et al. performed a standard (meta) genome-wide association study, which usually is applied to the genome of the host. However, they applied it to our "other" genome in the GI tract, based 
on deep shotgun sequencing of the gut microbial DNA from 345 Chinese individuals and identified and validated approximately 50 highly relevant type-2-diabetes-associated (T2D) markers. Although T2D was a significant factor for explaining the variation in the examined gut microbial samples, again, only a small fraction (3.8\%) of the gut microbial genes (at the relative abundance level) were associated with T2D in an individual. In general T2D-enriched functional markers were typically involved in the Kyoto Encyclopedia of Genes and Genomes (KEGG) categories of membrane transport. At the module or pathway level, the gut microbiota of T2D patients showed enrichment of functions related to membrane transport of sugars, xenobiotics degradation and metabolism, and functions relating to gut oxidative stress responses, which are consistent with the findings by ${ }^{194}$. In contrast, genes associated with butyrate biosynthesis were decreased in relative abundance, which indicates a decline in butyrate-producing bacteria ${ }^{195}$. These data raises the possibility that there is a 'functional dysbiosis', rather than there being a specific microbial species that has a direct association with T2D pathophysiology.

Recently Le Chatelier et al. sequenced the metagenomes of 292 Danish individuals and reported a bimodal distribution of bacterial genes in the obese individuals ${ }^{196}$. The low and high gene count groups (LGC, HGC) contained on average 380.000 and 640.000 genes, respectively, in line with lower or higher microbial richness as measured by HITChip analysis. Based on reference genome mapping of the metagenome reads, at the phylum level, LGC individuals were characterized by a higher relative abundance of Proteobacteria and Bacteroidetes, whereas the fecal microbiome of HGC individuals was enriched with Verrucomicrobia, Actinobacteria and Euryarchaeota. At the species level, HGC individuals showed higher prevalence of presumed anti-inflammatory species, such as Faecalibacterium prausnitzii, whereas LGC subjects had higher relative abundance of potentially pro-inflammatory Bacteroides and Ruminococcus gnavus. Nevertheless the vast majority (90\%) of the 120,723 genes that were significantly differing in relative abundance between the LGC and HGC gene individuals could not be assigned to a known bacterial genome and these genes were clustered into species like groups using a gene abundancebased approach. The taxonomy based conclusions were in concordance with the function analysis. LGC individuals had a higher abundance of peroxidase, catalase and TCA modules, suggesting increased capacity to handle exposure to oxygen/oxidative stress and they showed a reduced hydrogen and methane production potential, combined with increased hydrogen sulfide formation potential. In contrast, HGC individuals were characterized by a potentially increased production of organic acids - including lactate, propionate and butyrate - combined with a higher hydrogen production potential. Overall, this suggests that LGC individuals harbor an inflammation-associated microbiome. The authors tested significance of correlations of HGC and LGC individuals with several obesity associated anthropometric and biochemical variables, such as, BMI, weight, whole body fat, insulin resistance, hyperinsulinaemia, hyperlipidaemia, decreased HDL- 
cholesterol and markers of low grade inflammation and interestingly, all but weight and BMI were significantly correlated with gene count. Additionally, when they compared metagenomes between lean $(\mathrm{BMI}<25)$ and obese $(\mathrm{BMI}>30)$ only $\sim 16.000$ significantly different genes were found, indicating that the GI tract of obese and lean individuals differs less than that of the LGC and HGC individuals ${ }^{196}$. This again indicates that BMI is probably not an appropriate indicator for obesity. An accompanying paper demonstrated the same bi-modal gene richness distribution within a group of 11 overweight and 38 obese individuals that underwent a diet-induced weight-loss and weight-stabilization intervention ${ }^{197}$. Gene richness increased significantly in the LGC individuals and even remained higher than at baseline after the weight stabilization phase. This increase in gene richness was associated with an improvement of the metabolic status. Interestingly, increased consumption of fruit and vegetables and the decreased consumption of fishery products were associated with high bacterial richness at baseline and, although gene richness was not fully restored after the short-term intervention, these findings support the concept of the reported link between long term dietary habits and the structure of the gut microbiome ${ }^{102,198}$. It also suggests that a permanent change of the microbiome may be achieved by appropriate diet. Under the assumption that LGC individuals are less healthy ${ }^{196}$, this implies that the dietary changes might improve markers for obesity related metabolic disorders, either, through improvement of host parameters such as weight and whole body fat, modulation of the microbiome, or quite plausible, a combination of both. Nevertheless, microbial richness might have predictive value in the efficacy of dietary treatments.

Ferrer et al. reported concordance with some features outlined in the preceding paragraphs, when groups are stratified by BMI, such as a high functional similarity of the obese and lean microbiome in two subjects ${ }^{106}$ and a skewed B:F ratio. However, they also performed a simultaneous metaproteomics analysis, which provided evidence that the actual functional contribution of members of the Bacteroidetes and Firmicutes phyla was similar in both the "lean" and "obese" subject. This suggests that only taxonomic profiling and metagenomics might lead to an incomplete picture, because presence does not equal activity. So, although the 'functional dysbiosis' hypothesis by Qin et al and the LGC and HGC groups reported by Le Chatelier et al. (which was supported by both composition and metagenomics data) are showing that we are unravelling the dynamics and mechanisms underlying the role of the microbiome in obesity and its related metabolic complications, the observations by Ferrer et al. highlight the importance of a multiple perspectives approach through integration of compositional, metagenomic, but also functional analyses to identify the actual players. These functional tools will be addressed in the following chapter. 


\section{Community activity: what are they doing and who's doing what?}

As metagenomics can be considered as cataloguing genes, other meta-omics approaches, such as metatranscriptomics and metaproteomics which, respectively, use RNA and proteins as targets, are better suited to gain insight into the activity and functionality of the microbes in an ecosystem.

\section{Metatranscriptomics}

Environmental metatranscriptomics retrieves environmental mRNAs from a microbial ecosystem to assess which genes are expressed in that community at a given point in space and time. In addition, phylogenetic profiling of the mRNA-derived sequences may reveal the actual mechanisms by which individual members impact on the intestinal ecosystem ${ }^{199}$. The diversity of the microbiome has been the subject of many metagenomic studies but only a few have focused on the in situ activity of the microbiome in the human intestine. Initially, whole genome cDNA microarrays were used to study the activity of specific species in different GI ecosystems. For example Mahowald et al. performed whole-genome transcriptional analysis of colonic RNA from a simplified microbiome model in germ free mice, consisting of two species from the Firmicutes (Eubacterium rectale) and Bacteroidetes (Bacteroides thetaiotaomicon) ${ }^{200}$. They found that B. thetaiotaomicron adapted to the presence of $E$. rectale by up-regulating expression of a variety of polysaccharide utilization loci encoding numerous glycoside hydrolases, and by signalling to the host to produce mucosal glycans that $B$. thetaiotaomicon, but not $E$. rectale, can access. In turn, E. rectale was shown to adapt to $B$. thetaiotaomicron by decreasing production of its own glycan-degrading enzymes, increasing expression of selected amino acid and sugar transporters and facilitating glycolysis by reducing levels of NADH, partly via generation of butyrate from acetate, which in turn is used by the gut epithelium. This experiments elegantly illustrated niche specialization and functional redundancy of different members of the GI tract microbiome.

In another study, a comparative metatranscriptome profiling study of the fecal microbiome using the fingerprinting method: complementary DNA amplified fragment length polymorphism (cDNA-AFLP) revealed highly divergent expression profiles between two healthy subjects with considerable fluctuations in time. Genes encoding proteins involved in carbohydrate metabolism were being dominantly expressed ${ }^{201}$. Recently, the fecal metatranscriptome of monozygotic twin pairs was analyzed by next generation sequencing of RNA (RNA-seq) and metagenomics, revealing a higher relative expression, compared to the metagenomics data, of genes from KEGG pathways involved in carbohydrate metabolism, and vitamin metabolism/biosynthesis. Taxonomic mapping at genus level also revealed a two to three-fold higher relative expression, by members of the genera Parabacteroides, Bacteroides, Alistipes, Blautia, Methanobrevibacter and Coprococcus 
and a lower relative expression of Bifidobacterium (10-fold), Eubacterium (nine-fold), Collinsella and Dorea (five-fold) ${ }^{185}$.

Although metatranscriptome analysis is a powerful tool to determine gene expression, studies are faced with technical challenges, including the low recovery rates of high quality mRNA from environmental samples, the need for quenching procedures due to the short half-life times of microbial mRNA, and separation of mRNA from other RNA species ${ }^{110}$, ${ }^{202}$. Nonetheless, the advent of RNA sequencing by ultrahigh throughput NGS technologies has mitigated some of the technical issues by the sheer number of reads generated. Unfortunately, mRNA transcripts are far less stable than the actual produced proteins, and hence they do not necessarily represent their microbial function. Nonetheless, instantaneous inventories of mRNA pools are highly informative about ongoing ecologically relevant processes and fluctuations. However, only replicated, manipulative experiments will fully leverage the value of metatranscriptomes. That is, to reveal the microbes that perceive a specific environmental change and the metabolic pathways they invoke to respond to $\mathrm{it}^{203}$.

Such studies in humans would be faced with several challenges such as impracticalities associated with temporal and/or spatial sampling or instantaneous quenching of freshly voided fecal samples. The use of effluent from ileostomists (subjects without a colon), greatly reduces the aforementioned problems and has been employed by Leimena $e t$ al. to validate an RNA-seq metatranscriptome analysis pipeline ${ }^{204}$ and Zoetendal et al. to deduce an ecological model of the microbiome composition and function in the small intestine by combining several complementary culture independent approaches. They identified functions, such as PTSs, that are overrepresented in the small intestine, compared to fecal metagenomes. Moreover, an RNA-seq based metatranscriptome analysis supported high level in-situ expression of genes encoding PTSs and carbohydrate active enzymes. Overall, these findings suggest that rapid uptake and fermentation of available carbohydrates contributes to maintaining the microbiome in the human small intestine.

Another highly suitable platform for replicate gastrointestinal metatranscriptome analysis include dynamic computer controlled models that mimic the human GI tract, as they are highly stable, lack host feedback loops that could interfere with replicates, facilitate accessibility and temporal and spatial sampling ${ }^{56}$, which might be crucial as mRNA levels are naturally fluctuating.

Although no studies have been performed targeting the metatranscriptome of lean and obese individuals, the high level expression of PTS-encoding genes in the small intestine, an environment where simple sugar uptake is paramount, the enrichment of the same genes in metagenomic datasets from the GI tract of obese individuals and/or type 2 diabetes patients ${ }^{194,195}$ might indicate a higher quantity of polysaccharides reaching the large intestine of obese patients through the diet. Nevertheless, validating this through an mRNA bases functional analysis would undoubtedly be challenging as fecal metatranscriptomics 
are still faced with a host of technical challenges. However, technologies to study the fecal metaproteome are maturing as outlined in the following chapter, and could provide a useful alternative to know what the microbes are doing.

\section{Metaproteomics}

Metaproteomics is defined as the large-scale characterization of the entire protein complement of an environment, at a given point in time ${ }^{205}$, and it holds several benefits over metatranscriptomic approaches. Proteins are the actual catalysts of biochemical reactions and, as the half-life a typical bacterial protein is about two orders of magnitude longer than that of mRNA, most proteins persist in a bacterial cell long after mRNAs that encoded them have been degraded. Therefore mRNA transcripts do not necessarily represent the microbial function which is ultimately mediated by proteins ${ }^{203}$. Hence, proteome-based analyses can be expected to eventually provide a better and more accurate view of the functionality of the intestinal microbiome, especially when fecal samples are used as proxy to describe microbiota activity.

Initial metaproteomics analysis of fecal samples employed two-dimensional gel electrophoresis to highlight the temporal development of gut microbial proteins during the first days of infant life $\mathrm{e}^{206}$. However, current standard non-gel-based metaproteomics experiments typically comprise of four basic steps: (1) sample preparation including protein extraction, purification, and concentration; (2) protein denaturation and reduction; (3) protein (or peptide) separation, enzymatic digestion, and mass spectrometry (MS) analysis; and (4) protein identification based on the obtained MS and/or liquid chromatographyMS/MS data ${ }^{207}$. Common high-throughput spectral interpretation algorithms use peptide spectrum matching to link the raw data obtained from a mass spectrometer to large listings of peptides that are possibly represented in the data. The generation of theoretical spectra from peptides present in the database and matching them with the obtained data forms the basis of these algorithms ${ }^{208}$. In order to properly identify the spectra, one needs to know very accurately which peptides one can expect. However, the complex gut microbial proteome is far from defined. One method to overcome this problem is to exploit the current developments in metagenomics. Verberkmoes et al. performed the first large-scale investigation of the human microbial metaproteome using this approach, revealing highly abundant proteins in0volved in translation and carbohydrate and energy metabolism ${ }^{209}$. Another interesting observation was a clear discrepancy in the distribution of clusters of orthologous groups (COG) categories between the metaproteome and the metagenome, emphasizing the advantages of using a metaproteomic, over a metagenomic approach, although it has to be noted that the metagenome and metaproteome were from different individuals ${ }^{209}$. Although metaproteomics still suffers from the limited coverage of the present databases and a potentially high false discovery rate, the catalogue of genes from intestinal origin is rapidly increasing, and recently an iterative workflow based on a synthetic metaproteome was developed. It improved the number of identified peptides by 
two fold, at a false discovery rate of only $1 \%{ }^{210}$. This highly dynamic workflow allows capitalizing on the developing metagenomic databases and was used to study the composition and temporal stability of the intestinal metaproteome from 3 individuals, revealing a stable common functional core that is mainly involved in carbohydrate transport and degradation ${ }^{211}$

The identified metaproteome can also be used to link phylogeny to function in situ, by focusing on the proteome of phylogenetically deeply rooted microbial taxa, as these stand out in the blast analyses. An example are Akkermansia muciniphila-like bacteria which are the only known intestinal representative of the deeply rooted Verrucomicrobia and capable of using mucin as carbon and nitrogen source $\mathrm{e}^{212}$. An analysis of proteins assigned to $A$. muciniphila from the metaproteome of a single subject revealed a specific COG distribution of the peptides. Apart from the obvious housekeeping functions, the largest COG groups included proteins predicted to be involved in carbohydrate transport and metabolism as well as amino acid transport and metabolism. As these proteins include various mucinases, it testifies for predicted activity and function of A. muciniphila-like bacteria in the intestinal tract.

Ferrer et al. reported the first and so far only comparative metagenomic and metaproteomic investigation of GI tract microbial communities in fecal samples taken from an obese and lean adolescent ${ }^{106}$. The "lean" and "obese" metagenomes were assigned to COG protein families and KEGG pathways, yet neither profile exhibited prominent overall differences. They analyzed the metaproteome and, although the identified proteins only captured a small portion of the predicted coding capacity of the intestinal microbiome, the majority of the spectra could be assigned to a taxonomic annotation at phylum level, based on highly similar homologues, allowing them to evaluate the differences between the contributions of particular groups of organisms and identify metabolically active members. This led to some remarkable observations. For example, proteins from the 'lean' sample were mainly assigned to Bacteroidetes (81\%), whereas this phylum represented only about $20 \%$ (based on 16S rRNA genes) of the total community, followed by proteins assigned to Firmicutes $(12 \%)$ and, to a minor extent, Actinobacteria and Proteobacteria (approximately $3 \%$ in total). In contrast, in the 'obese' sample the major taxon accounting for observed proteins was the Firmicutes phylum $(56 \%)$ in agreement with its dominance in the total bacteria ( $94 \%$ 16S rRNA gene). In turn, the Bacteroidetes accounted for only $3.2 \%$ of the total community, yet their contribution to the total protein pool was $42 \%$ in the obese subject. Based on this data, the authors suggested that minor bacterial taxa may play a significant active role in overall GI metabolism in 'lean' and 'obese' individuals, with Bacteroidetes members possibly playing a major active role in both 'lean' (rRNA gene/protein abundance ratio of 1:4) and 'obese' (rRNA gene/protein abundance ratio of 1:13) GI $\operatorname{tracts}^{106}$. Conversely, this high Bacteroidetes activity was not confirmed by Kolmeder et al. who reported that the majority of assigned proteins (60\%) belonged to the phylum Firmicutes, 
which had an average abundance of $86 \%$ as based on 16S rRNA gene targeted microarray analysis. In contrast, the high proportion (33\%) of peptides from Actinobacteria differed from the phylogenetic analysis (only $2 \%)^{211}$. The relative share of Bacteroidetes was lower in the peptide (6\%) than in $16 \mathrm{~S}$ rRNA data $(11 \%)$. About $0.2 \%$ of the peptides with at least a phylum assignment could be attributed to the Proteobacteria, which was approximately 10 -fold lower than their abundance (2\%). So, although the compositional data was obtained using very different approaches, (clone libraries and a phylogenetic microarray respectively), using different protein extraction protocols (which could offer potential explanations for the major differences found between both studies) and employing only a very small number of samples, these studies provide functional evidence that variable combinations of species, with a relatively stable community gene repertoire, could presumptively fulfil overlapping and/or complementary functional roles required by the host. In this scenario minor bacterial taxa can still be significantly active contributors.

Metaproteomics compared to other functional genomics approaches such as metatranscriptomics has the advantage that actual and stable gene products can be studied. Although metaproteomic approaches in the human gastrointestinal microbiome field are faced with several challenges and therefore, are still in a pioneering phase, they promise to be increasingly comprehensive because of the exponential progress in the generation of relevant genomic and metagenomic datasets joined with recent advances in the analysis protocol $^{211}$. This has been recently reviewed by Kolmeder et $a l^{213}$.

\section{Conclusions and future prospects}

Currently it is still unclear which bacterial groups play a role in the development of obesity, in humans. This might partly be explained by the heterogeneity in, genotype, lifestyle, diet and the complex etiology of Obesity and its Associated Metabolic Disorders (OAMD). Also, the use of different target-groups/organisms between qPCR and FISH based studies in the past, might have blurred the subject somewhat. Moreover, several studies have revealed the diet to be a major factor influencing GI microbial composition, which has been shown to skew the composition towards a hypothesized obesogenic microbiome (high Firmicutes, low Bacteroidetes), independent of obesity mainly in mice ${ }^{165-167}$, but also humans ${ }^{179}$. A recent study in rats actually implies bile acids, the secretion of which is enhanced to facilitate lipid digestion, as a factor that modulates the GI microbial composition towards the above-mentioned proposed obesogenic microbiome ${ }^{214}$. Although the debate on a correlation between the B:F ratio and BMI is still ongoing, it has been suggested that this correlation is particularly observed in severely obese individuals (BMI $>35^{174}$ ) as are the overweight subjects in the initial publication, by Ley et al. that proposed this phylum-wide shift, and whose subjects exhibited BMIs between 30 and $43^{104}$. 
Nevertheless, recent research on this matter has shown a conceptual shift, from stratifying lean and obese subjects based on BMI, which is actually a very poor marker for OAMD ${ }^{176}$, ${ }^{177}$, to selecting categories based on both anthropometric (BMI, weight, total body fat) and biochemical variables (insulin resistance, hyperinsulinaemia, hyperlipidaemia, decreased HDL-cholesterol and markers of low grade inflammation). The results have been promising and an OAMD associated microbiome now seems to be characterized by a lower microbial diversity $^{36,90,174}$ or gene/species richness ${ }^{194,196,197}$ (the latter being confirmed by both metagenomics and phylogenetic microarray analysis) and an increased abundance of potential pro-inflammatory species, such as members of the Proteobacteria phylum and the genus Bacteroides ${ }^{90,174,196}$. Overall, whereas comparative metagenomic analysis of OAMD and healthy individuals has revealed high functional uniformity between the two groups ${ }^{36}$. 106, 194-196, the OAMD associated microbiome has been shown to be enriched for genes encoding proteins involved in membrane transport of sugars ${ }^{36,194,195}$, xenobiotics degradation and metabolism ${ }^{194,195}$, and functions related to gut oxidative stress responses ${ }^{195}$, 196 and in contrast, decreased butyrate biosynthesis ${ }^{195}$. In turn, a potentially increased production of organic acids, including lactate, propionate and butyrate, was shown for the High Gene Count group (associated with a healthy phenotype), proposed by Le Chatelier et $a l .{ }^{196}$.

Now that correlative studies of the GI tract microbiome with OAMD are focusing on more homogenous subpopulations, and technological advances have allowed for very deep interrogation of the GI tract microbiome, with many more subjects than previously possible, a more consistent picture seems to emerge. All in all, the results from 16S rRNA gene targeted compositional analyses, metagenome-based profiling through reference genome mapping and function analysis of metagenomic data, suggest that an OAMD associated microbiome can be characterized by a potential pro-inflammatory composition, with less potential for the production of SCFAs and butyrate in particular. Although this link between SCFA producing species has been proposed in many compositional studies, coupling 16S rRNA based data to actual microbial function is difficult due to the functional overlap in metabolism between different groups of microorganisms. Together with the fact that a very large fraction of the reads in metagenomic datasets are still unassignable to a known genome, the issue of actual biomarker organisms is bound to remain controversial. So, for a more thorough understanding of the potential impact of these unknown species on host metabolism, databases with a better coverage are needed, which is currently being facilitated by the completion of ongoing reference genome sequencing projects for GIassociated bacterial species.

Although the aforementioned features of an OAMD associated microbiome illustrate the potential of a consensus on this paradigm, they remain correlative and not causative. This is highlighted by observations from the scarce functional analyses that investigated the actual metabolically active members of the fecal microbiome. These demonstrate that minor 
bacterial taxa can still be significantly active contributors and vice versa, creating awareness that compositional profiling and metagenomics studies might not provide the full picture and even though it remains outside the scope of this review, metabolomics comprises another complementary tool that could be used to validate metabolic pathwaybased predictions $\mathrm{s}^{36,106,211,215,216}$. Therefore, to gain more insight in the dynamics and mechanisms underlying the role of the microbiome in the maintenance or development of OAMD and to study causation as well as correlation, several factors are of paramount importance: 1) a multi-perspectives approach that targets both composition and function to reveal the actual active players, and 2) well defined human cohorts, followed longitudinally, to reduce or control for the impact of the various confounding factors that have been shown to affect GI microbial composition, such as diet. 



\section{Chapter 3}

Gut microbiota and body weight in healthy school-aged children: The KOALA Birth Cohort Study

Catherine A. Mbakwa*, Gerben D. A. Hermes*, John Penders, Paul H. M. Savelkoul, Carel Thijs, Pieter C. Dagnelie, Monique Mommers, Erwin G. Zoetendal,

Hauke Smidt and Ilja C.W. Arts

*These authors contributed equally to this work 


\begin{abstract}
Objective: To examine the association between intestinal microbiota composition and body weight in children at school age.

Methods: Within the KOALA Birth Cohort Study faecal samples were collected, and height and weight were measured at age 6-7 years. Faecal microbiota composition of 295 children was determined using the Human Intestinal Tract Chip. Elastic net was used to select genus-like bacterial groups (amongst a total of 130) related to anthropometric outcomes (age- and sex- standardized weight and BMI z-scores, overweight defined as $\mathrm{BMI} \geq 85$ th percentile). Multiple linear and logistic regression were used to associate selected bacterial groups with anthropometric outcomes while adjusting for confounders.
\end{abstract}

Results: Prevotella melaninogenica et rel., Prevotella oralis et rel., Dialister and uncultured Clostridiales II (UCII) accounted for $26.1 \%$ of the variation in microbiota composition. The following bacterial groups were inversely associated with anthropometric outcomes: Akkermansia ( $\mathrm{p}=0.030$ for BMI; $\mathrm{p}=0.009$ for weight); Sutterella wadsworthia et rel. $(\mathrm{p}=0.008$ for BMI; $\mathrm{p}=0.038$ for overweight $) ;$ Bryantella formatexigens et rel. $(\mathrm{p}=0.050$ for weight; $\mathrm{p}=0.027$ for overweight), and Burkholderia ( $\mathrm{p}=0.017$ for weight). Streptococcus bovis et rel. was positively associated with overweight $(\mathrm{p}=0.013)$. Among bimodal bacterial groups, high abundance of UCII was inversely associated with all three outcomes (adj $\beta$ 0.22 ; $95 \% \mathrm{CI}-0.42$ to -0.02 for BMI and weight; adjOR 0.28 ; $95 \% \mathrm{CI} 0.10$ to 0.79 for overweight). High abundances of Prevotella melanogenica et rel. and Prevotella oralis et rel. were inversely associated with overweight (adjOR $0.21 ; 95 \% \mathrm{CI} 0.07$ to 0.68 , and adjOR $0.20 ; 95 \% \mathrm{CI} 0.06$ to 0.64 , respectively). Microbial diversity and richness, and Bacteroidetes:Firmicutes ratio were not significantly associated with any of the outcomes.

Conclusions: In the largest population-based study on childhood gut microbiota and body weight so far, we found both new and previously identified bacterial groups to be either positively or negatively associated with overweight. Further research should elucidate their role in energy metabolism. 


$\begin{array}{ll}\text { Abbreviations } \\ \text { HITChip } & \text { Human Intestinal Tract Chip } \\ \text { BLAST } & \text { Basic Local Alignment Search Tool } \\ \text { BMI } & \text { Body Mass Index } \\ \text { CI } & \text { Confidence Interval } \\ \text { SD } & \text { Standard deviation } \\ \text { qPCR } & \text { quantitative Polymerase Chain Reaction } \\ \text { OR } & \text { Odds ratio } \\ \text { Adj } \beta & \text { Adjusted regression coefficient }\end{array}$

\section{Introduction}

The worldwide prevalence of childhood overweight has been growing at an alarming rate during the past decades. Overweight children are highly prone to become overweight adults and are at high risk of developing comorbidities such as cardiovascular diseases, type 2 diabetes, stroke, metabolic syndrome, several types of cancer, and osteoarthritis ${ }^{217}$. As such, the prevention and treatment of childhood overweight remains an important public health goal.

While excessive energy intake and insufficient physical activity are the main drivers of childhood overweight and obesity, recent research has suggested that other factors such as the gut microbiota may also be involved. The gut microbiota is highly diverse in composition and plays an important role in human physiology, metabolism, nutrition and immune function ${ }^{96,121}$. Evidence from human and animal studies suggests that the gut microbiota may contribute to the development of overweight via mechanisms involving increased energy harvest,${ }^{48}$ regulation of host metabolism, ${ }^{218}$ and the activation of innate immunity ${ }^{219,220}$. In humans, obesity has been associated with a reduced Bacteroidetes to Firmicutes (B:F) ratio in some studies, ${ }^{36,104,174,221}$ whereas others reported the opposite ${ }^{171}$, 222, 223 or no association at all ${ }^{170,17,224-226}$. Associations between specific bacteria (e.g. Bacteroides fragilis, ${ }^{227}$ Bifidobacterium $\mathrm{spp}$, Staphylococcus spp, ${ }^{228}$ Akkermansia muciniphila $^{229}$ and Faecalibacterium prausnitzii) ${ }^{230}$ or Archaea (e.g. Methanobrevibacter smithii) $)^{231}$ and obesity in humans have also been reported, although the identified microbial groups vary greatly between studies. Furthermore, a lower gut microbial species and gene richness and diversity in overweight as compared to normal weight subjects has been shown in several but not all studies ${ }^{36,164,196,229}$.

These inconsistent findings might be attributable to the use of miscellaneous methods to assess the gut microbiota, often enumerating specific taxa rather than using broad 16S rRNA gene surveys or metagenomics. Moreover, studies vary greatly in the populations 
considered, their designs, and the degree of control for potential confounding factors such as lifestyle and diet. In human observational studies, carefully controlled data analysis is essential $^{232}$. Perturbations in microbial diversity and community structure in adults with overweight and obesity may be partly due to long-term dietary habits or physiological changes in these subjects ${ }^{196}$. As such, exploring the association between the gut microbiota and variation in BMI and weight in early life, prior to or close to the onset of overweight, might provide additional insights. We therefore aimed to investigate the relation between gut microbiota composition and body weight in a group of 295 well-characterized schoolaged children.

\section{Material and methods}

\section{Subjects and study design}

The current study is conducted within the context of the KOALA Birth Cohort Study in the Netherlands. The design of the KOALA study has been described in detail elsewhere ${ }^{233}$. Briefly, a total of 2834 pregnant women were recruited, at 34 weeks of gestation, from October 2000 until December 2002. Healthy pregnant women with a conventional lifestyle $(\mathrm{N}=2343)$ were recruited from an on-going cohort study on the aetiology of pregnancyrelated pelvic girdle pain in the Netherlands ${ }^{234}$. To enhance the contrast in lifestyle characteristics, an additional 491 pregnant women with alternative lifestyles with regards to dietary habits (organic food choice), child rearing practices, vaccination schemes and/or use of antibiotics, were recruited through organic food shops, anthroposophist doctors and midwives, Steiner schools and dedicated magazines ${ }^{233}$.

A subgroup of 1,204 parents was asked for consent for a home visit for anthropometric measurements and to collect a single faecal sample from the child at the age of 6-7 years. This subgroup comprised of participants who had home visits for blood collection from the mother during pregnancy and/or the child at age 2 years, and who were still active participants (Supplementary Figure 1). Faecal samples were obtained for $\mathrm{n}=669$ children. Exclusion criteria for the current study were: prematurity (infants born before 37 weeks of gestation), twins, abnormalities linked to growth (such as Down's syndrome, Turner syndrome, Fallot's tetralogy, multiple disabilities, and cystic fibrosis), faecal samples with transport times exceeding 3 days, or lack of data on dietary intake. A total of 295 children were finally included in the present study; all being Caucasians. Written informed consent was given by all parents, and the study was approved by the Medical Ethics Committee of Maastricht University and the National Ethical Committee for Medical Research. 


\section{Data collection and outcome measures}

\section{Faecal collection}

Faecal samples of the children were collected by the parents at home upon receipt of a faeces tube with a spoon attached to the lid (Sarstedt, Nürmbrecht, Germany) together with instructions for collection. A faecal sample was collected and sent to the laboratory by mail. After arrival, samples were 10-fold diluted in peptone/water (Oxoid CM0009) containing $20 \%$ (vol/vol) glycerol (Merck, Darmstadt, Germany), and stored at $-80^{\circ} \mathrm{C}$ until further analysis.

\section{Faecal DNA isolation and microbiota profiling}

DNA isolation from faecal samples has been described in detail elsewhere ${ }^{109}$. Briefly, DNA was isolated using a combination of Repeated-Bead-Beating (RBB) and column purification. DNA concentration and purity were assessed with a Nanodrop 1000 spectrophotometer (Thermo Fisher Scientific, Wilmington, USA). DNA was then stored at $-20^{\circ} \mathrm{C}$ pending microbial analysis, which was performed using a previously described and benchmarked $^{155,159,235}$ custom made, phylogenetic microarray, the Human Intestinal Tract Chip (HITChip) ${ }^{159}$. After DNA extraction, the full-length 16S rRNA gene was amplified, followed by in vitro transcription and labelling of the resultant RNA with $\mathrm{Cy} 3$ and $\mathrm{Cy} 5$ before hybridization to the array ${ }^{159}$. Each sample was hybridized at least twice to ensure reproducibility, and raw signal intensities were normalised as previously described ${ }^{93}$. For the data analysis, hybridization signals were summarized to 130 genus-like phylogenetic groups ( $>90 \% 16 \mathrm{~S}$ rRNA gene sequence similarity) referred to as species and relatives ('et rel.' $)^{159}$. The $\log 10$-transformed signals were used as a proxy for bacterial abundance. B:F ratio was calculated by dividing total hybridisation signal intensities for Bacteroidetes by Firmicutes. Diversity of the microbiota was quantified using Shannon's diversity index based on non-logarithmic oligo-level signals as implemented by the $\mathrm{R}$ package vegan $^{236}$. Probes were counted in each sample to measure richness, by using an $80 \%$ quantile threshold for detection.

\section{Anthropometric outcomes}

Height and weight were measured during home visits by trained research assistants at the age of 6-7 years, with the children wearing only their underwear. Height (in millimetres) was measured using a portable stadiometer (Leicester height measure) and weight (in grams rounded off to $100 \mathrm{~g}$ ) using a digital scale (HE-5, CAS Corp., East Rutherford, NJ, USA). BMI was calculated as weight divided by height squared. The BMI, weight and height measurements were then converted into age- and gender-specific z-scores using the children from the Dutch National Growth Study as the reference population ${ }^{237}$. BMI z-scores were used both as continuous and dichotomous outcomes: without overweight vs. with overweight (BMI z-score $\geq 1.04$, corresponding to the $85^{\text {th }}$ percentile) ${ }^{238}$. 


\section{Potential confounders}

At 14 and 34 weeks of gestation, pregnant women received questionnaires regarding, amongst others, family size, pre-pregnancy height and weight, and weight gain during pregnancy. Two weeks after childbirth, data was collected from obstetric reports, and questionnaires were completed by the mothers to obtain information on gestational age, birth weight and gender of the child. Food frequency questionnaires (FFQs) were filled out by the parents to report the dietary habits of their children at the age of 5.0 0.6 years (mean \pm standard deviation (SD)). Included confounders are listed in Supplementary Table 1 .

\section{Statistical analysis}

Characteristics of the study population are presented as mean $\pm \mathrm{SD}$ for continuous variables, and proportions for categorical variables. The microbiota profile, summarized into 130 genus-like phylogenetic groups was obtained for the 295 subjects. Using the statistical software package Canoco $5^{239}$ redundancy analysis (RDA), a multivariate canonical ordination analysis method, was performed to determine how much variation in species composition was explained by the anthropometric outcomes and potential confounders.

\section{Selection of potentially relevant bacterial groups}

In order to prevent issues with multi-collinearity and multiple testing when performing regression analyses with large numbers of determinants, we first selected genus-like bacterial levels potentially associated with childhood (over)weight using the elastic net regularization proposed by Zou \& Hastie ${ }^{240}$. Elastic net is a method that utilises the ridge $(\alpha=0)$ and lasso $(\alpha=1)$ penalties to perform both shrinkage and automatic variable selection simultaneously. It also addresses the problem of multi-collinearity by encouraging a grouping effect, where strong correlated genus-like bacterial levels are kept in the model. The data was divided at random into two parts: a training set with 197 observations, and a test set with 98 observations (Figure 1). Model fitting and tuning parameters ( $\lambda$, and s) selection was done on the training set by performing a 10 -fold cross-validation. The first tuning parameter $(\lambda)$ plays a role in variable selection, whereas the second (s) captures correlated predictors at the same time. The chosen grid values were: $(0,0.01,0.1,1,10$, and 100$)$ for $\lambda$, and ( 0 to 1 dividing on a scale of 0.1 ) for $\mathrm{s}$ as previously suggested ${ }^{240}$. The most parsimonious model was obtained using the combination of the two tuning parameters corresponding to the smallest mean-squared prediction error based on the cross-validations performed on the training set. Validation of the model performance was then performed by comparing the computed prediction mean squared error (for continuous outcomes) on both the training and test set. In the case of the binary outcome (overweight (yes/no)) validation of the performance of model prediction was done using area under the curve.

In addition, we a priori planned to examine the association between bacterial groups that exhibited a strong bimodal abundance distribution (calculated using potential analysis ${ }^{241}$ 
with the early warnings $\mathrm{R}$ package $\mathrm{e}^{242}$ ) and anthropometric outcomes. These bimodal bacterial groups were previously described Lahti et al. as potential tipping elements in the ecosystem, ${ }^{93}$ and might serve as indicators of the community state and its link with (over)weight. We selected bacterial groups that showed consistent evidence for multimodality with bootstrap support $\geq 68 \%$.

\section{Regression analysis}

First we performed unadjusted regression models (further referred to as Model 1) to analyse the association of each individual selected genus-like bacterial group (as identified by elastic net), bimodal bacterial groups (tipping elements), microbial richness and diversity, and B:F ratio, with the outcomes (linear regression for BMI and weight z-scores, logistic regression for overweight yes/no).

Second, we performed the same analyses while adjusting for potential confounders considered a priori (Model 2). We investigated whether the association between the intestinal microbiota composition and outcomes differed between the two different recruitment channels (alternative and conventional) by including a recruitment groupexposure interaction term in the models. This interaction was significant only for B:F ratio with regards to BMI and weight z-scores (data not shown).

Finally, we performed multiple regression models including all selected bacterial groups, the bimodal bacterial groups, and Archaea (M. smithii (yes/no) and M. stadtmanae (yes/no)) while adjusting for the potential confounders (Model 3). Archaea were added because in our previous study, ${ }^{231}$ the presence of Archaea, specifically Methanobrevibacter smithii, was associated with childhood weight development.

Maternal weight gain during pregnancy was the only confounding variable with $\geq 5 \%$ missing values. Multiple imputations were done for this variable to assess whether results obtained from the imputed data deviated from the non-imputed. This was done using the Markov chain Monte Carlo (MCMC) method for multiple imputations. Results obtained from combined imputed datasets $(\mathrm{n}=10)$ were comparable with those of the original nonimputed data, hence final analyses were done without imputation. Analyses were performed in R V.3.1.3 using the following packages: elastic net (for continuous outcomes) and glmnet (for binary outcomes), and SAS version 9.3 (SAS Institute, Cary NC). Significance level was set at $<0.05$. 


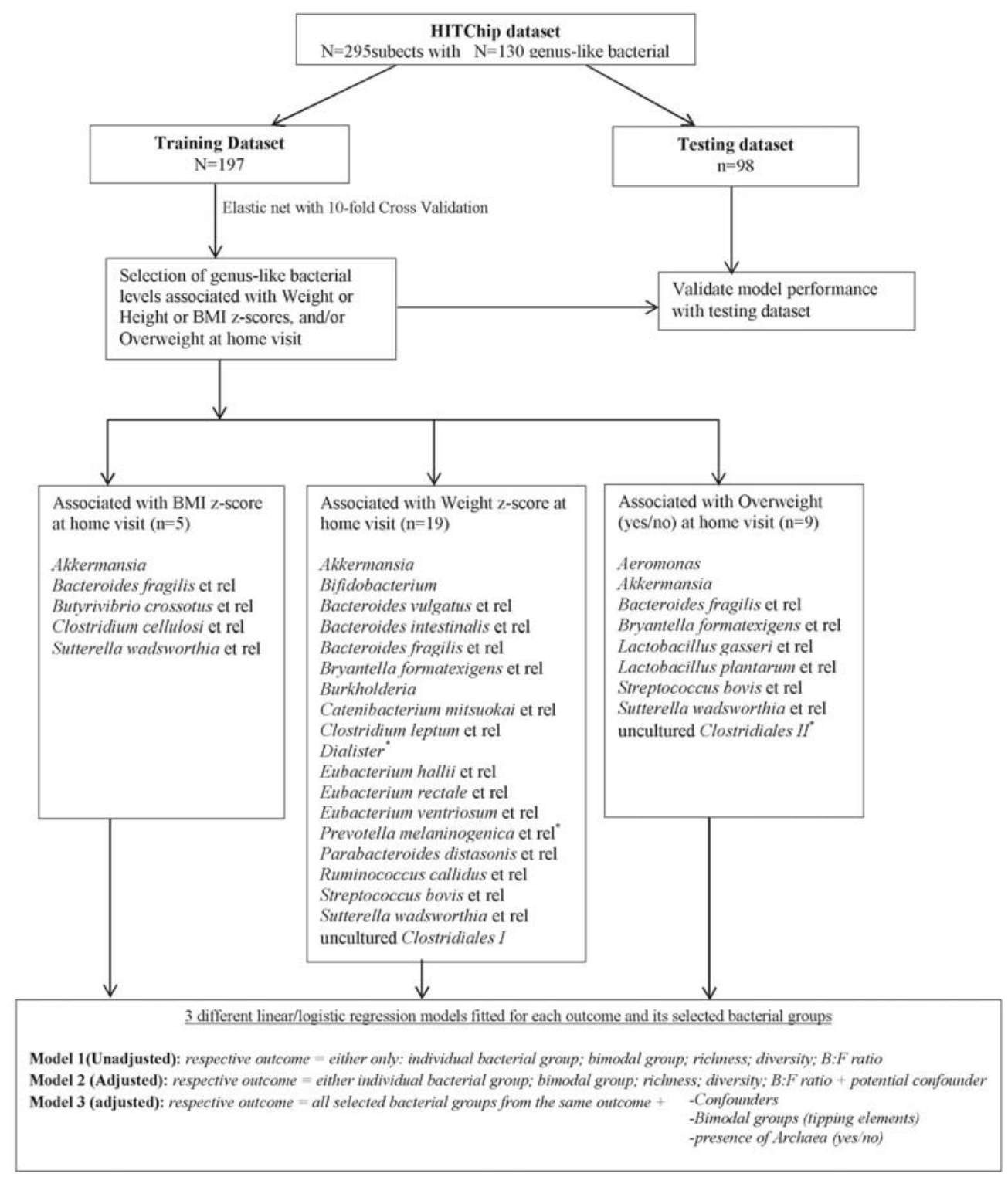

Figure 1: Schematic overview of the bacterial groups that were selected by elastic net for subsequent regression analysis.

*Genus-like bacterial groups identified as having a bimodal abundance distribution were analysed as dichotomous variables in the linear and logistic regression models 


\section{Results}

General characteristics of the total KOALA population and of the current study population are presented in Table 1. A total of 295 subjects (148 (50.2\%) boys and 147 (49.8\%) girls) with a mean age of 7.4 years (SD 0.8) were eligible for the present study (Supplementary Figure 1). There were 27 (9.8\%) overweight children.

The gut microbiota of the children was dominated by Prevotella melaninogenica et rel., Bacteroides vulgatus et rel., Ruminococcus obeum et rel., Faecalibacterium prausnitzii et rel., and Bifidobacterium (Figure 2). RDA was performed to explore what percentage of variability of gut microbiota composition was explained by the anthropometric measures. The percentage of variability explained by each anthropometric measure under consideration was very low: $0.5 \%(\mathrm{p}=0.15), 0.6 \%(\mathrm{p}=0.06)$, and $0.5 \%(\mathrm{p}=0.09)$ by BMI $z$ scores, weight z-scores, and overweight, respectively (data not shown). We observed strong or moderate bootstrap support for a bimodal distribution in the abundance of Prevotella melaninogenica et rel., Prevotella oralis et rel., Dialister, and uncultured Clostridiales II (UCII), but not for Bacteriodes fragilis et rel., and uncultured Clostridiales I (UCI). A distribution plot for each of the bacterial groups exhibiting bimodality (classified as low or high) and their cut-off or tipping points are illustrated in Supplementary Figure 3. The cumulative abundance of the four bimodal groups accounted for $26.1 \%$ of the variation, with largest variation explained by Dialister, Prevotella oralis et rel. and melaninogenica et rel., and their co-correlating groups (Figure 3).

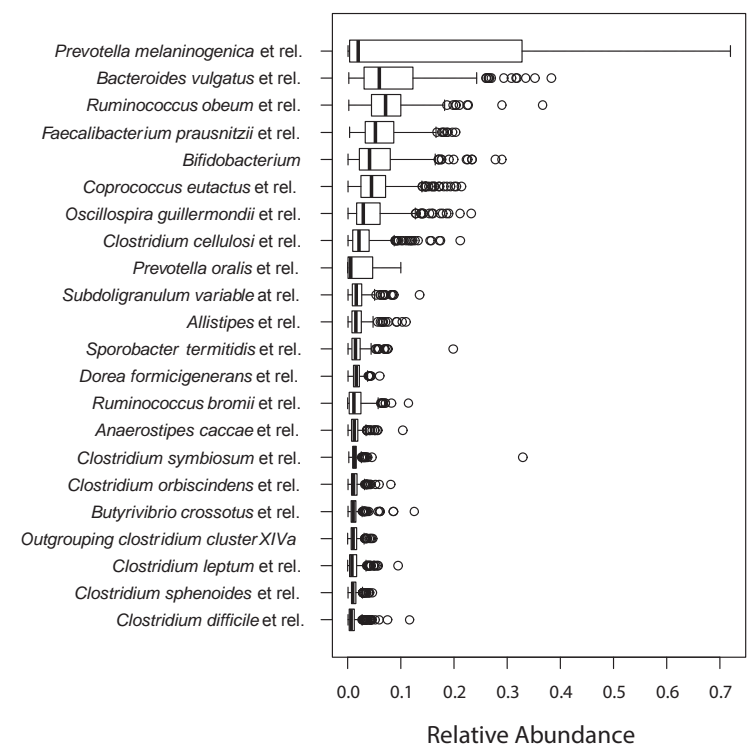

Figure 2: Boxplots representing the relative abundance of the 21 most abundant taxa (mean relative abundance $>1 \%$ ). 


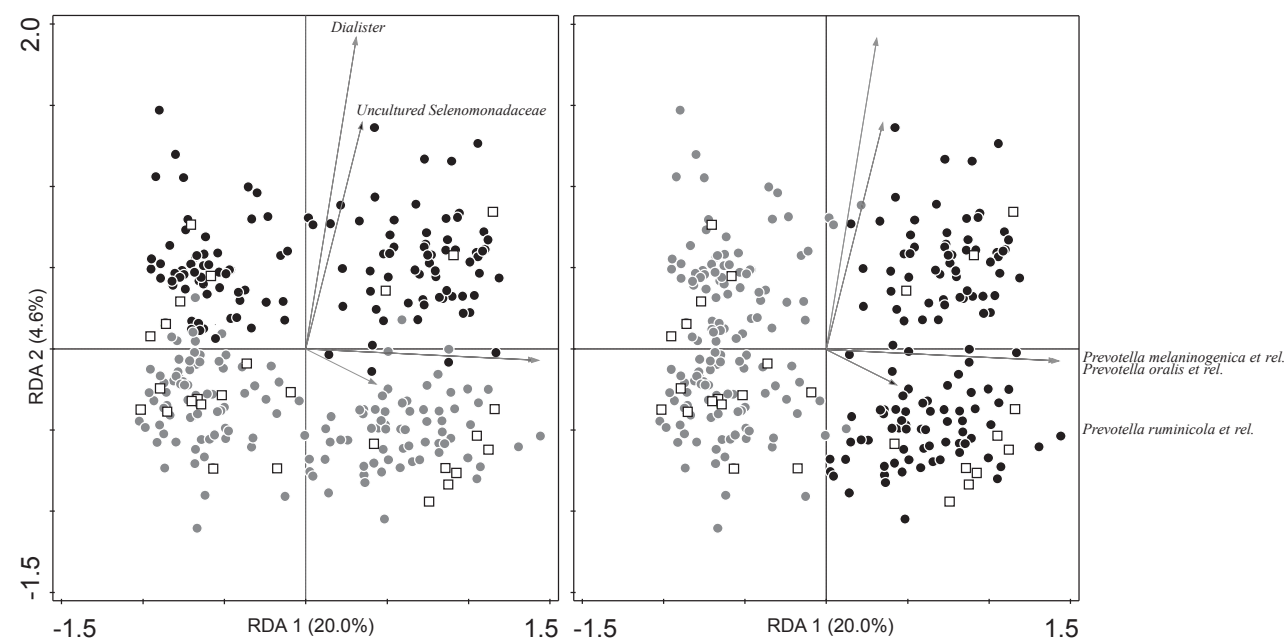

Figure 3. Abundance of bimodal taxa shape overall composition. RDA visualising microbiota composition of all faecal samples $(\mathrm{n}=295)$ coloured by high (black dots) and low (grey dots) abundance of Dialister (left panel), and Prevotella melaninogenica (right panel). Overweight individuals are represented as squares. The direction of the arrows depicts the abundance of the bimodal bacterial groups as well as their co-correlating groups. Length of the arrows is a measure of fit for the species.

Considering the bimodal bacterial groups, a high abundance of UCII was inversely associated with BMI- and weight z-score, $(\operatorname{adj} \beta-0.22 ; 95 \%$ CI -0.42 to -0.02 for both; Table 2 ) and overweight (adjOR 0.28 ; 95\% CI 0.10 to 0.79 ; Table 3 ) in the adjusted analysis. Higher abundances of Prevotella melanoginica et rel. (adjOR 0.21; 95\%CI 0.07 to 0.68 ) and Prevotella oralis et rel. (adjOR $0.20 ; 95 \% \mathrm{CI} 0.06$ to 0.64 ) were inversely associated with overweight (Table 3). Regarding microbial richness, diversity, and B:F ratio, we only found a borderline significant inverse association between microbial diversity and overweight in the unadjusted linear regression model. Overall, microbial richness and diversity and B:F ratio appeared not be associated with anthropometric outcomes in the childhood population studied.

We subsequently examined whether specific bacterial groups were associated with the anthropometric outcomes under study. Elastic net was applied to the 130 genus-like bacterial groups for each outcome measure separately. A total of 5, 19, and 9 weightassociated bacterial groups were identified as main effects with regards to BMI z-scores, weight z-scores, and overweight, respectively (Figure 1). The elastic net coefficient paths for these bacterial groups with respect to each outcome are presented in Supplementary Figures 2a-c. The selected bacterial groups were included as continuous variables in the regression models, except for Prevotella melanogenica et rel. and Dialister spp. (weight zscores), and UCII (overweight) as they were already identified as having a bimodal abundance distribution and as such were analysed as dichotomous variables.

After adjusting for confounders (Table 2, Model 2), BMI z-score was inversely associated with Akkermansia $(\mathrm{p}=0.030)$, and Sutterella wadsworthia et rel $(\mathrm{p}=0.008)$. Similar results 
were obtained from Model 3, which included all five pre-selected genus-like bacterial groups, other microbial groups (Archaea, bimodal bacterial groups), and confounders (Supplementary Table 2). Weight z-scores were inversely associated with Akkermansia $(\mathrm{p}=0.009)$, Burkholderia $(\mathrm{p}=0.017)$, and Bryantella formatexigens et rel. (borderline significant; $\mathrm{p}=0.050$ ) after adjusting for confounders (Table 2, Model 2). In the model with all 19 pre-selected bacteria adjusted for confounders plus other microbial groups (supplementary Table 2, Model 3); only Akkermansia, and Bryantella formatexigens remained inversely associated with weight z-scores. With regards to overweight, Sutterella wadsworthia et rel. $(\mathrm{p}=0.038)$, and Bryantella formatexigens et rel. $(\mathrm{p}=0.027)$, were inversely associated with overweight in the adjusted analyses. In contrast, Streptococcus bovis et rel. ( $\mathrm{p}=0.013)$ was positively associated with overweight. In the model with all nine pre-selected bacteria, other microbial groups, and confounders (Supplementary Table 2, Model 3), only Akkermansia remained significantly inversely associated with overweight. Overall, Akkermansia, Sutterella wadsworthia et rel., and Bryantella formatexigens et rel. were consistently associated with the three anthropometric outcomes, even after adjusting for the presence of other gut microbial groups and confounders. 
Table 1. General characteristics of the KOALA Birth Cohort and the study population

\begin{tabular}{|c|c|c|}
\hline & KOALA Birth Cohort Study & Study Population \\
\hline Age at home visit (years) & - & $7.4 \pm 0.8$ \\
\hline \multicolumn{3}{|l|}{ Anthropometric data measured at home visit } \\
\hline \multicolumn{3}{|l|}{ Overweight: n (\%) } \\
\hline yes & - & $27(9.8)$ \\
\hline no & - & $268(90.2)$ \\
\hline BMI z-scores & - & $-0.16 \pm 0.89$ \\
\hline Weight z-scores & - & $-0.20 \pm 0.96$ \\
\hline \multicolumn{3}{|c|}{ Time of last antibiotic course prior to home visit: $\mathbf{n}(\%)$} \\
\hline No antibiotic use in the previous year & - & $248(85.2)$ \\
\hline Greater than 4 weeks ago & - & $36(12.4)$ \\
\hline Less than 4 weeks ago & - & $7(2.4)$ \\
\hline Age at faecal sample collection (years) & - & $7.3 \pm 0.8$ \\
\hline Child's total physical activity (hrs/week) & - & $9.4 \pm 4.5$ \\
\hline \multicolumn{3}{|l|}{ Recruitment group } \\
\hline Conventional & $2343(82.7)$ & $218(73.9)$ \\
\hline Alternative & $491(11.3)$ & $77(26.1)$ \\
\hline \multicolumn{3}{|l|}{ Maternal educational level $(n, \%)^{d}$} \\
\hline Low & $289(10.7)$ & $21(7.2)$ \\
\hline Middle & $1060(39.4)$ & $113(38.8)$ \\
\hline High & $1341(49.9)$ & $157(54.0)$ \\
\hline Maternal pre-pregnancy weight (Kg) & $67.7 \pm 13.1$ & $68.3 \pm 11.5$ \\
\hline Maternal weight gain in pregnancy (Kg) & $14.3 \pm 5.1$ & $14.5 \pm 4.8$ \\
\hline \multicolumn{3}{|l|}{ Place and mode of delivery: $n(\%)^{c}$} \\
\hline Vaginal delivery at home & $1187(44.8)$ & $143(49.0)$ \\
\hline Vaginal delivery in the hospital & $1149(43.4)$ & $120(41.1)$ \\
\hline Caesarean section in the hospital & $311(11.8)$ & $29(9.9)$ \\
\hline \multicolumn{3}{|l|}{ Maternal smoking in late pregnancy: $\mathbf{n}(\%)$} \\
\hline Yes & $200(7.1)$ & $12(4.1)$ \\
\hline No & $2634(92.9)$ & $283(95.9)$ \\
\hline Total Household size & $4.3 \pm 0.8$ & $4.3 \pm 0.8$ \\
\hline Gestational age & $39.8 \pm 5.0$ & $39.8 \pm 3.7$ \\
\hline Birth weight (g) & $3503 \pm 512$ & $3605 \pm 466$ \\
\hline Breastfeeding duration (months) & $4.7 \pm 3.0$ & $6.0 \pm 4.4$ \\
\hline \multicolumn{3}{|l|}{ Gender: n (\%) } \\
\hline Male & $1451(51.2)$ & $148(50.2)$ \\
\hline Female & $1376(48.6)$ & $147(49.8)$ \\
\hline \multicolumn{3}{|l|}{ Child's dietary intake } \\
\hline Total energy intake (KJ) & $6173 \pm 1285$ & $6180 \pm 1217$ \\
\hline$\%$ energy intake from fats & $29.6 \pm 4.2$ & $29.7 \pm 4.2$ \\
\hline$\%$ energy intake from carbohydrates & $55.8 \pm 5.0$ & $55.7 \pm 4.8$ \\
\hline Total fibre intake $(\mathrm{g})$ & $15.3 \pm 4.0$ & $15.6 \pm 3.9$ \\
\hline
\end{tabular}

${ }^{\mathbf{a}, \mathbf{b}}$ Total may not sum up to 2834 and 295 respectively due to missings.

${ }^{c}$ Values are mean (standard deviation), unless indicated otherwise.

${ }^{\mathrm{d}}$ Low: primary school, preparatory vocational or lower general secondary school, Middle: vocational, higher general secondary and pre-university, High: higher vocational or academic. 


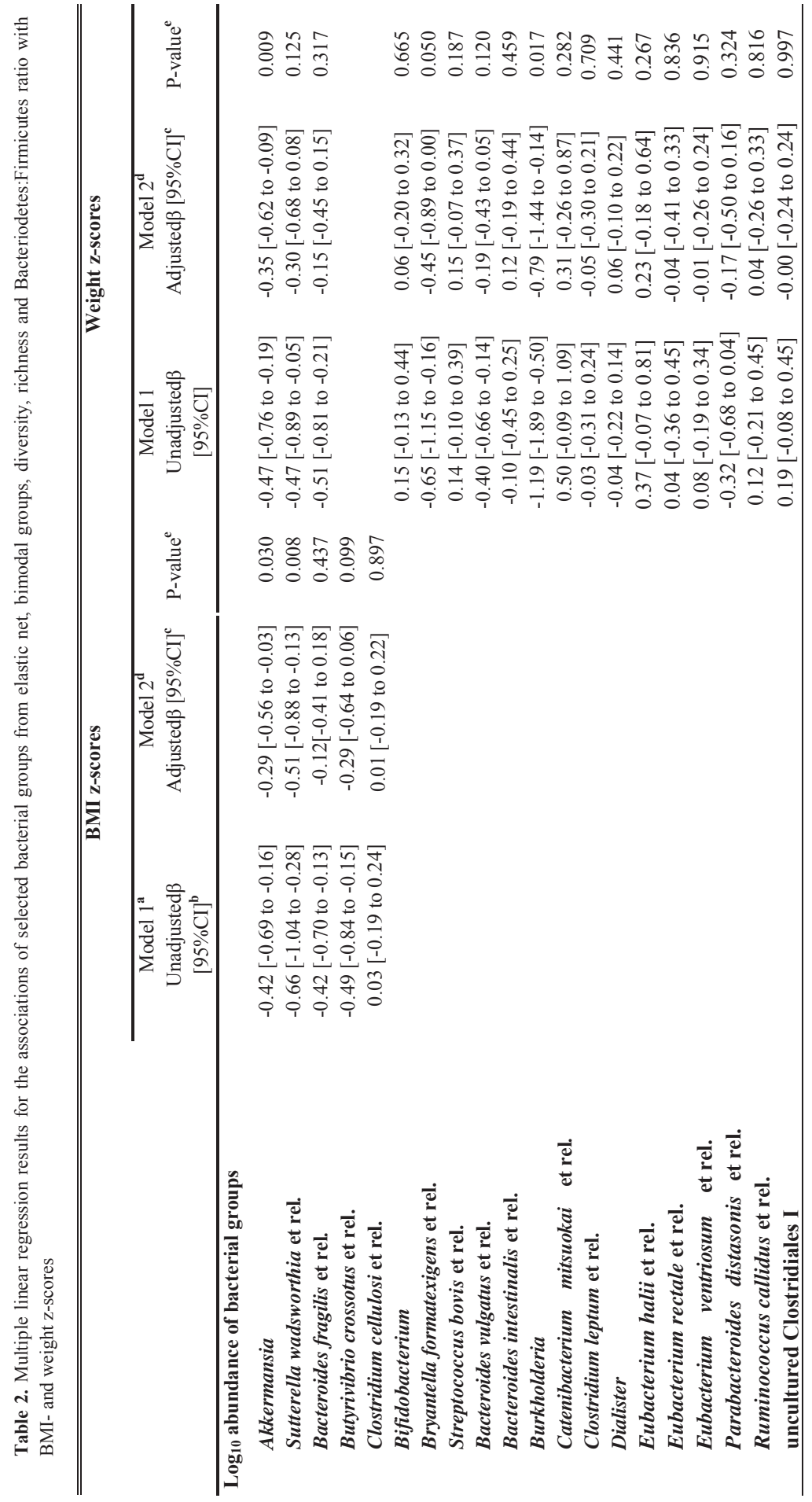




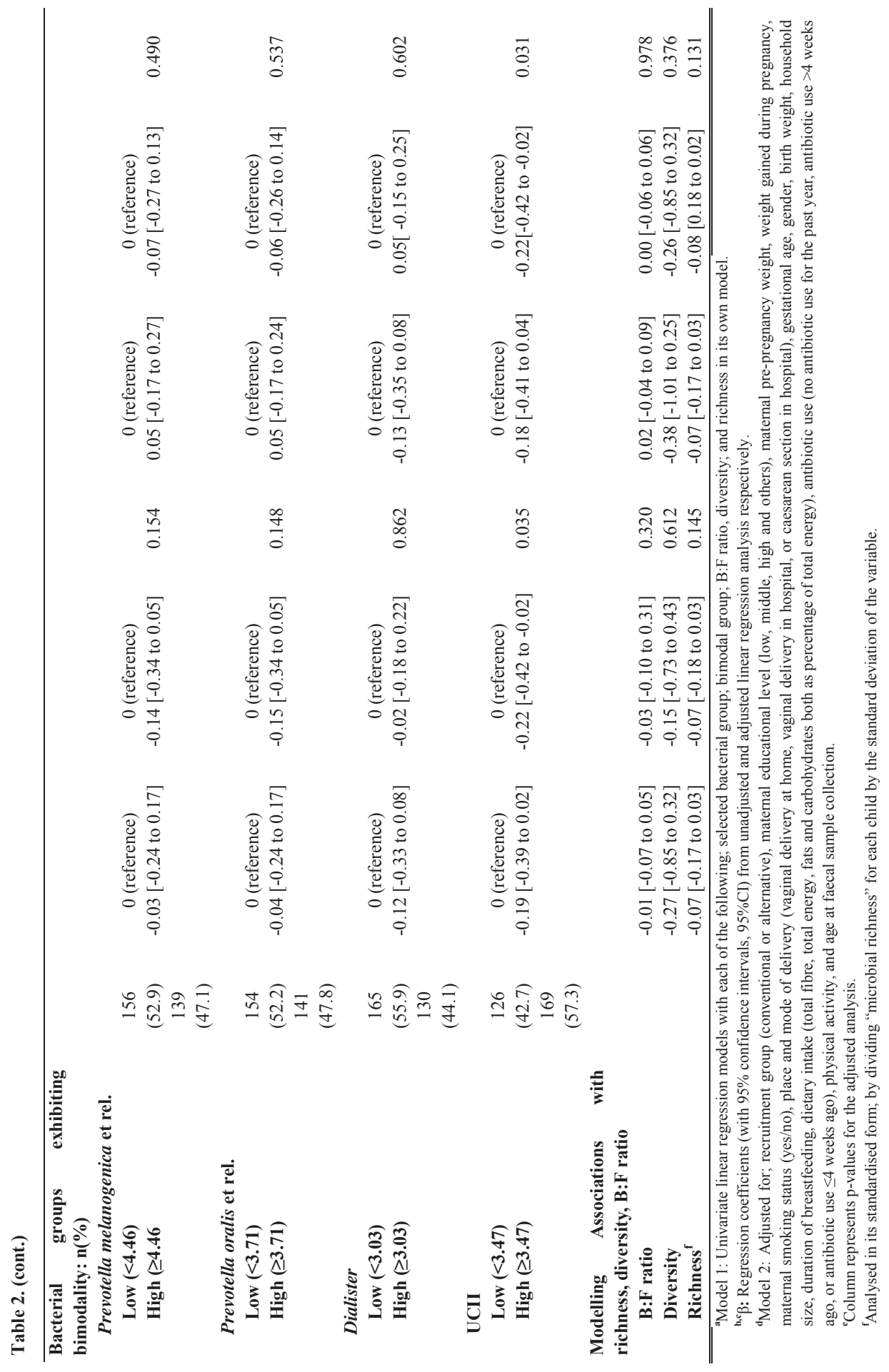




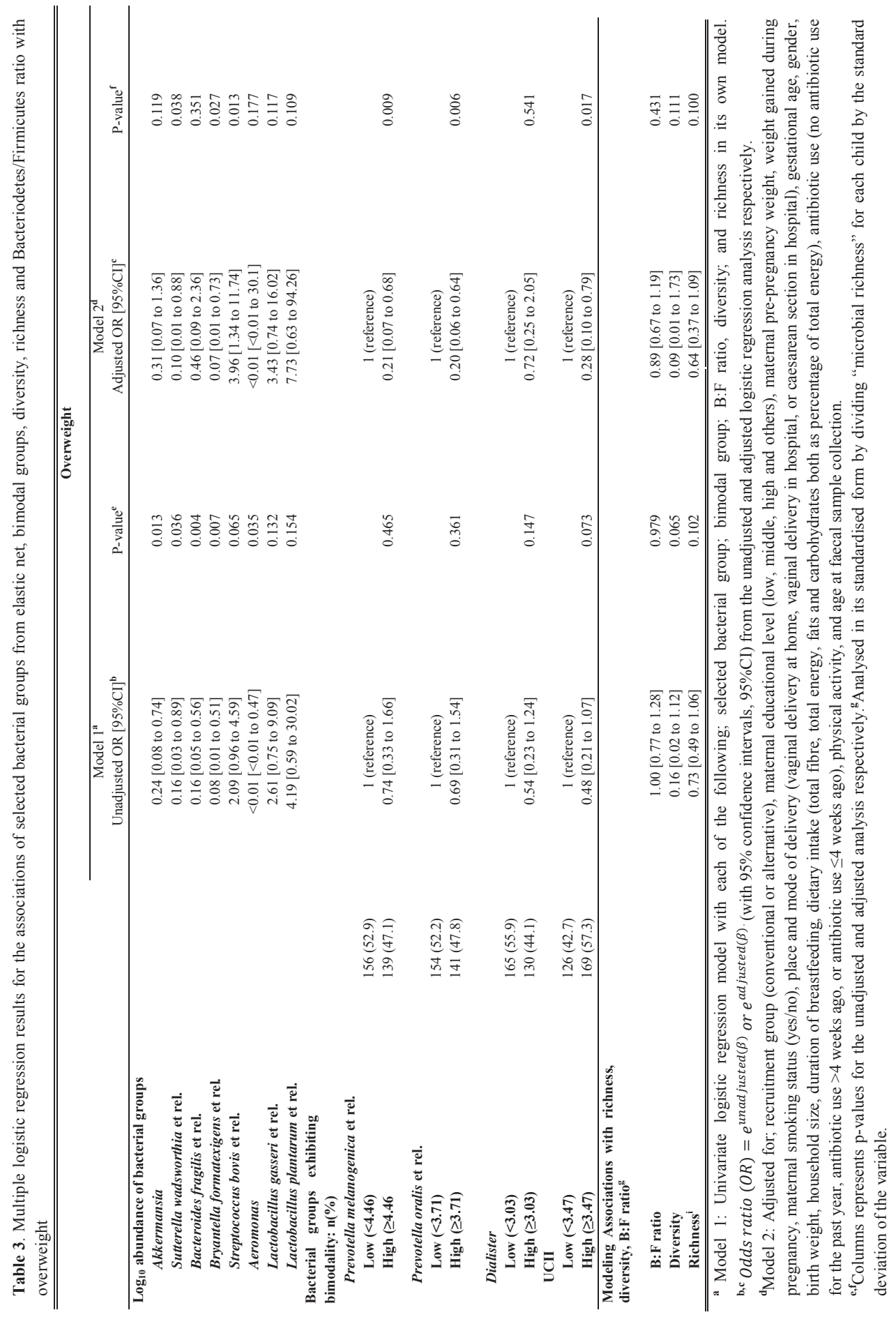




\section{Discussion}

Within a population of 295 school-aged children participating in the KOALA-study, we examined the association between microbiota composition and overweight. So far, this is the largest population-based study on childhood gut microbiota in relation to body weight. Moreover, the extensive data on lifestyle and diet allowed us to carefully control for potential confounding factors. The anthropometric outcomes and potential confounders explained very little variation in the overall microbiota composition; however, the abundances of several specific bacterial groups were consistently linked to BMI-, weight zscores, and overweight.

The gut microbiota of 6-7 year-old children is less well studied than that of adults, and it is still being debated whether the composition has reached a relatively stable, adult-like state soon after weaning ${ }^{29}$ or continues developing into teen years ${ }^{243}$. An in-depth comparison with adults is beyond the scope of this paper, however, it is noteworthy that we observed several bimodal distributed bacterial groups as well as their co-correlating species that were previously reported to be present in healthy adults, ${ }^{93}$ including UCII, Prevotella spp. (P. oralis et rel. and $P$. melaninogenica et rel.) co-correlating with $P$. ruminicola et rel., and Dialister with uncultured Selenomodaceae. Lack of support for bimodality of UCI in our study is also in concordance with Lahti et $a l,{ }^{93}$ who showed previously that UCI exhibits very clear shifting state probabilities associated with ageing, where the high abundant state was only observed above 40 years of age. Since the bootstrap support of bimodality for Bacteroides fragilis was only moderate in the study of Lahti et al., this bimodality might have been missed in our study due to the smaller sample size, age, health status, or other biological factors.

The abundance of the four bimodal groups explained $26.1 \%$ of the variation in species composition, compared to $<1 \%$ for each of the anthropometric outcomes. Therefore, we cannot confirm the often large-scale community shifts previously reported mainly from rodent studies ${ }^{48,164}$. In this group of healthy children within a relatively normal weight range, weight and associated parameters therefore did not seem to be major drivers of overall microbial composition or vice versa. This is in line with observations from Hollister et al., who also found that BMI failed to account for a significant proportion of the variation in gut microbial composition and function of American children at age 7-12 years $^{243}$. The B:F ratio was not associated with weight-related outcomes in the present study. Although previous studies have reported a lower B:F ratio in individuals with overweight or obesity as compared to normal weight individuals ${ }^{36,104,174,221,244,245}$ others studies found the opposite association, ${ }^{171,222,223}$ or analogous to our study, no association at all $^{170,173,224-226,230}$ 
A healthy gut microbial ecosystem is generally thought to be characterised by high microbial richness and diversity, presumed to indicate a more stable and resilient state of this ecosystem ${ }^{246}$. A lower richness/diversity of the gut bacterial communities has been reported in subjects with obesity, and overweight compared to normal weight individuals ${ }^{174}$, 196,247 . We also observed a tendency towards a lower gut microbial diversity in overweight compared to normal weight children. However, this association disappeared upon adjusting for confounders. This implies that differences in microbial diversity between obese and lean subjects, as observed in previous studies not comprehensively controlling for confounders may be overestimated. Altogether, our results confirm that the B:F ratio and diversity appear not to be a general feature distinguishing the normal and overweight human gut microbiota across populations ${ }^{47}$.

Although we did not find a significant association with the overall microbial community composition, we did observe associations between the anthropometric outcomes and the contrasting alternative stable states of specific bacterial groups that could potentially act as tipping elements of the whole gut microbial ecosystem. We could confirm the observation by Lahti et al. (2014) that UCII was inversely associated with BMI. We found an inverse association with BMI- and weight $\mathrm{z}$-scores, and with overweight. Likewise, higher abundances of $P$. oralis et rel. and $P$. melaninogenica et rel., were inversely associated overweight even after adjusting for other microbial groups that were also associated with overweight, but not with the other outcomes. In addition, using the elastic net statistical method of variable selection, ${ }^{240}$ we identified several bacterial groups, of which Akkermansia, Bryantella formatexigens et rel. and Sutterella wadsworthia et rel. were consistently associated with all three anthropometric outcomes. The inverse association between Akkermansia abundance and body weight is consistent with previous studies both in mice and humans ${ }^{169}, 229,248,249$. Although the precise mechanism through which Akkermansia influences host metabolism has not yet been fully elucidated, studies in mice have demonstrated that it is involved in the reduction of metabolic endotoxemia, which is characteristic of obesity and associated metabolic disorders, through the restoration of gut barrier function ${ }^{65}$. Bryantella formatexigens et rel. has been shown to ferment glucose to acetate in the presence of high formate concentrations ${ }^{250}$ and the production of acetate can result in appetite suppression, ${ }^{251}$ suggesting a mechanism by which these bacteria might be linked to lower weight. Sutterella wadsworthia et rel. has been suggested to a play a role in autism spectrum disorder in children, ${ }^{252}$ but its role in overweight development has not been reported before.

The strengths of this study compared to previous cross-sectional studies on the gut microbiota and childhood overweight are; its large sample size in combination with a broad and high resolution interrogation of the whole gut microbial community, and extensive adjustment for important confounding variables (especially diet and physical activity). This allowed us to select bacterial groups (out of a total of 130) that were associated with 
weight-related outcomes, without an a priori constraint on the target species. In addition, our study is one of the first to examine the association between microbiota composition and body weight in a population of mostly lean, healthy children. Previous studies focused on extreme categories of lean and obese individuals. As a consequence, differences in microbiota composition might be less prominent in our study as compared to these previous studies, but it provides insight in the role of the gut microbiota in the normal developing child.

A limitation of the present study was the transport time for faecal samples, which ranged from less than 1 to 3 days at ambient to room temperature. This might have affected the measurable diversity and structure of the bacterial communities. However, several previous studies have shown that the microbial diversity and composition of faecal samples is much more affected by inter-individual differences and biases in molecular techniques rather than differences in short-term storage conditions, including storage for up to 2 weeks at room temperature ${ }^{253,254}$. Furthermore, we could only assess the associations of the bacterial groups with weight outcomes at one time point, and as a result causality could not be established. However, only a few longitudinal studies have been published so far in this field ${ }^{227,255}$. Hence, large longitudinal cohort studies that characterise the gut microbiota at multiple time points and collect detailed data on important confounding variables (e.g. mode of delivery, diet, and physical activity) are needed to obtain an in-depth knowledge of the relation between gut microbiota dynamics and childhood weight development. The ongoing follow-up of the children in our study facilitates a future longitudinal investigation of our findings taking into account these important confounding factors.

In conclusion, weight-related outcomes failed to explain much of the observed variation in gut microbiota composition in our cohort of healthy children, suggesting that at least in this group of individuals, weight-related parameters (BMI and weight z-scores, and overweight) are not major drivers of microbial composition in the gut. Nonetheless, several specific bacterial taxa appeared to be consistently associated with weight-related outcomes. These include several bacteria that have previously been linked to weight-related outcomes (Akkermansia, UCII), as well as species that have not previously been linked to (over)weight such as Sutterella wadsworthia et rel. and Bryantella formatexigens et rel. In this regard, more detailed information on their functional role in energy metabolism will help to establish their importance for weight development. Our results provide new avenues with regards to bacteria in the gut of humans in relation to the increasing trend of overweight worldwide. 


\section{Supplemental material}

Supplementary Table 1. List of potential confounding variables

\begin{tabular}{|c|c|}
\hline Potential confounders & Variable description/label $^{\mathrm{a}}$ \\
\hline Recruitment group & $\begin{array}{l}\text { Categorical } \\
\qquad \quad 0 \text { - alternative } \\
\bullet \quad 1 \text { - conventional }\end{array}$ \\
\hline Maternal educational level & $\begin{array}{l}\text { Categorical } \\
\text { - } \quad 0 \text { - lower education } \\
\text { - } \quad 1 \text { - vocational education } \\
\text { - } \quad 2 \text { - higher general secondary/pre-university } \\
\text { - } \quad 3 \text { - higher vocational/academic education }\end{array}$ \\
\hline Maternal pre-pregnancy weight & Continuous \\
\hline Weight gained during pregnancy & Continuous \\
\hline Maternal smoking status & $\begin{array}{l}\text { Categorical } \\
\bullet \quad 0-\text { no } \\
\bullet \quad 1-\text { yes }\end{array}$ \\
\hline Place and mode of delivery & $\begin{array}{l}\text { Categorical } \\
\text { - } 0 \text { - vaginal delivery at home } \\
\text { - } 1 \text { - vaginal delivery in hospital } \\
\text { - } 2 \text { - caesarean section in hospital }\end{array}$ \\
\hline Gestational age & Continuous \\
\hline Gender & $\begin{array}{l}\text { Categorical } \\
\qquad \quad 0 \text { - male } \\
-\quad 1 \text { - female }\end{array}$ \\
\hline Birth weight & Continuous \\
\hline Household size & Continuous \\
\hline $\begin{array}{l}\text { Duration of breastfeeding } \\
\text { Child's dietary intake }\end{array}$ & Continuous \\
\hline Total energy intake (KJ) & Continuous \\
\hline$\%$ energy intake from fats & Continuous \\
\hline$\%$ energy intake from carbohydrates & Continuous \\
\hline Time of last antibiotic course prior to home visit & $\begin{array}{l}\text { Categorical } \\
\text { - } 0 \text { - no antibiotic use in the previous year } \\
\text { - } 1 \text { - greater than } 4 \text { weeks ago } \\
\text { - } 2 \text { - less than } 4 \text { weeks ago }\end{array}$ \\
\hline Physical activity & Continuous \\
\hline Age at faecal sample collection (years) & Continuous \\
\hline
\end{tabular}

${ }^{a}$ All zero values were treated as reference category for each categorical variable. 


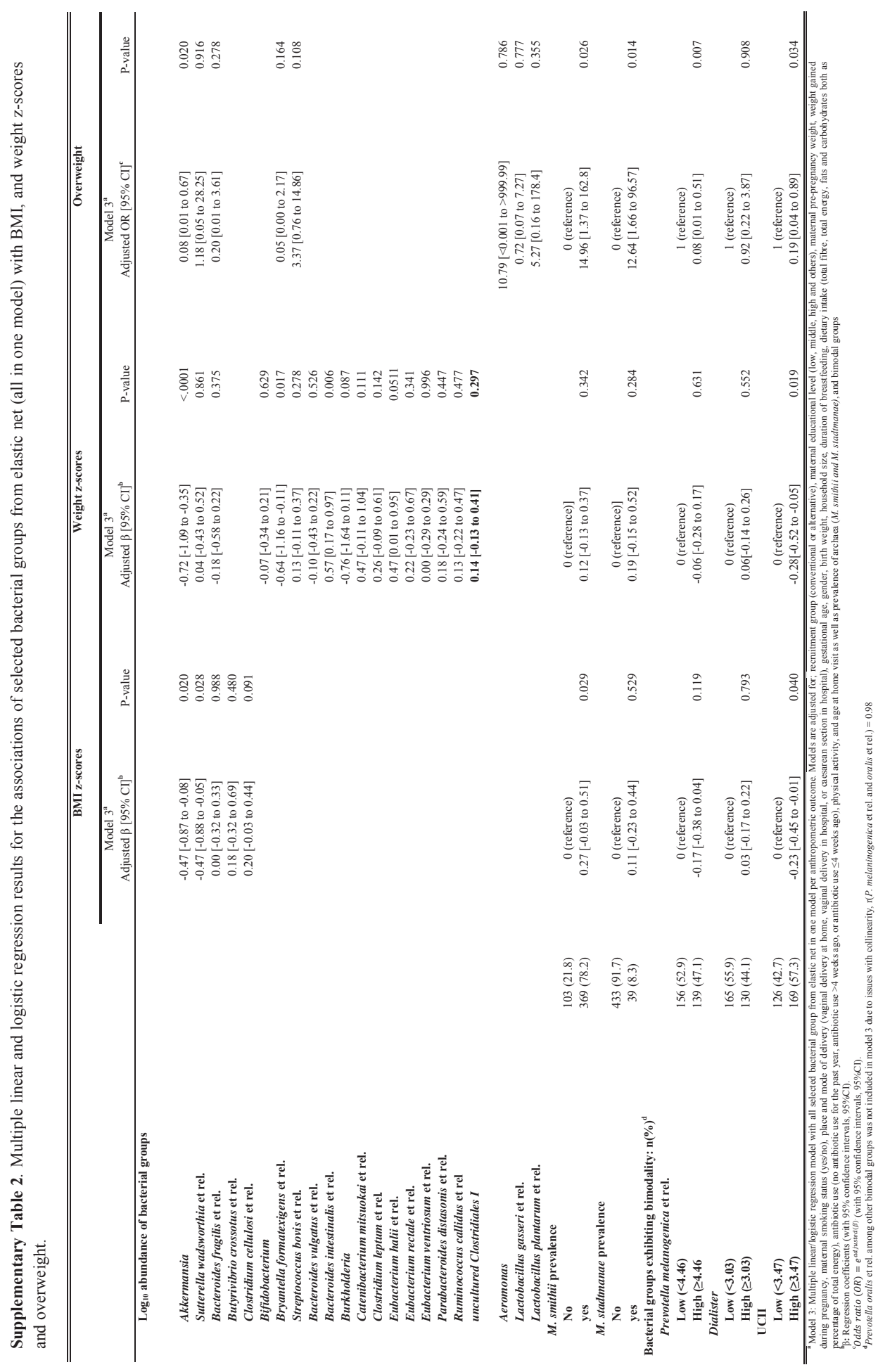




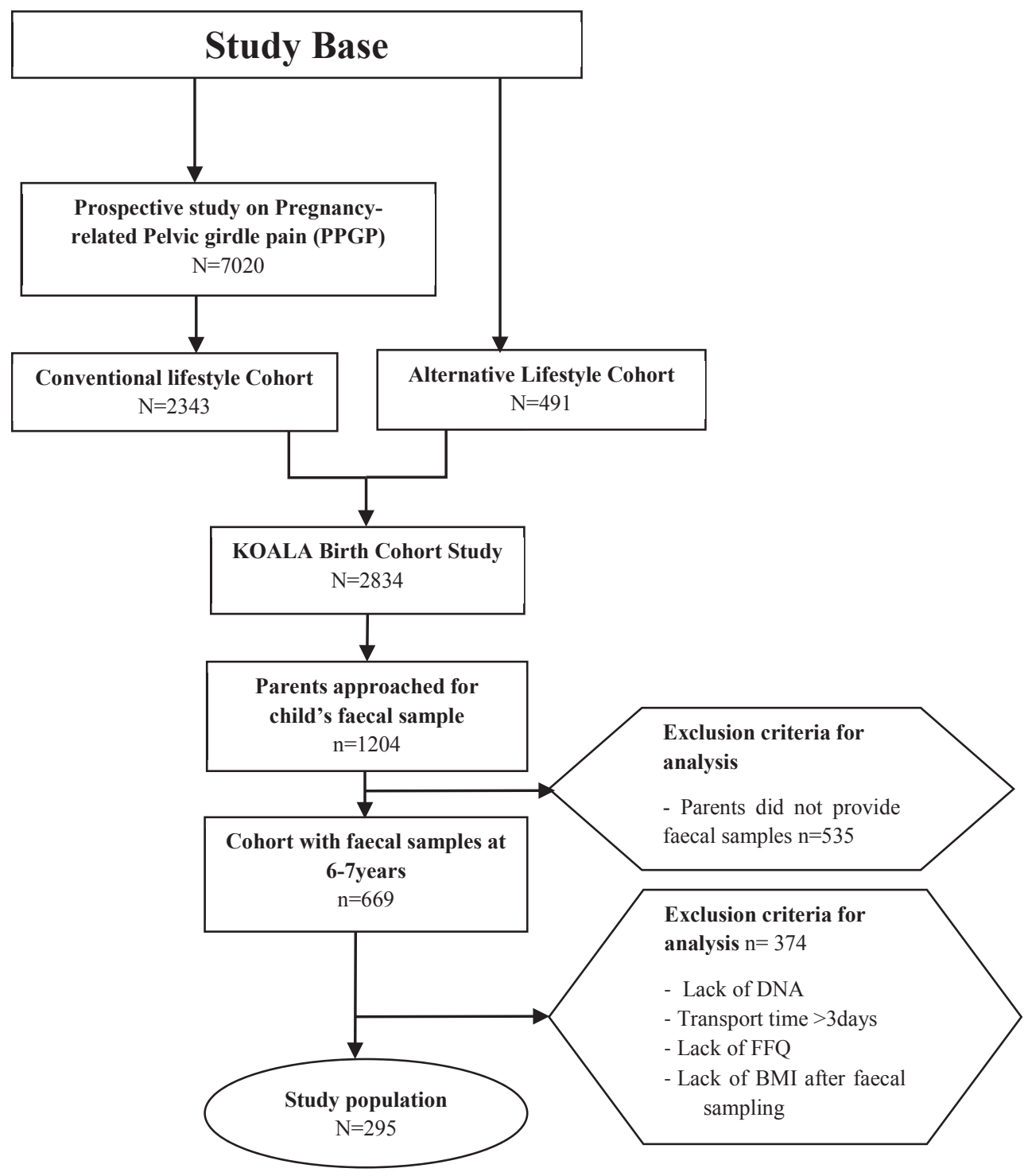

Supplementary Figure 1. Flow chart illustrating how the present study population of 295 children was obtained from the initial KOALA cohort of 2834 healthy pregnant women. 

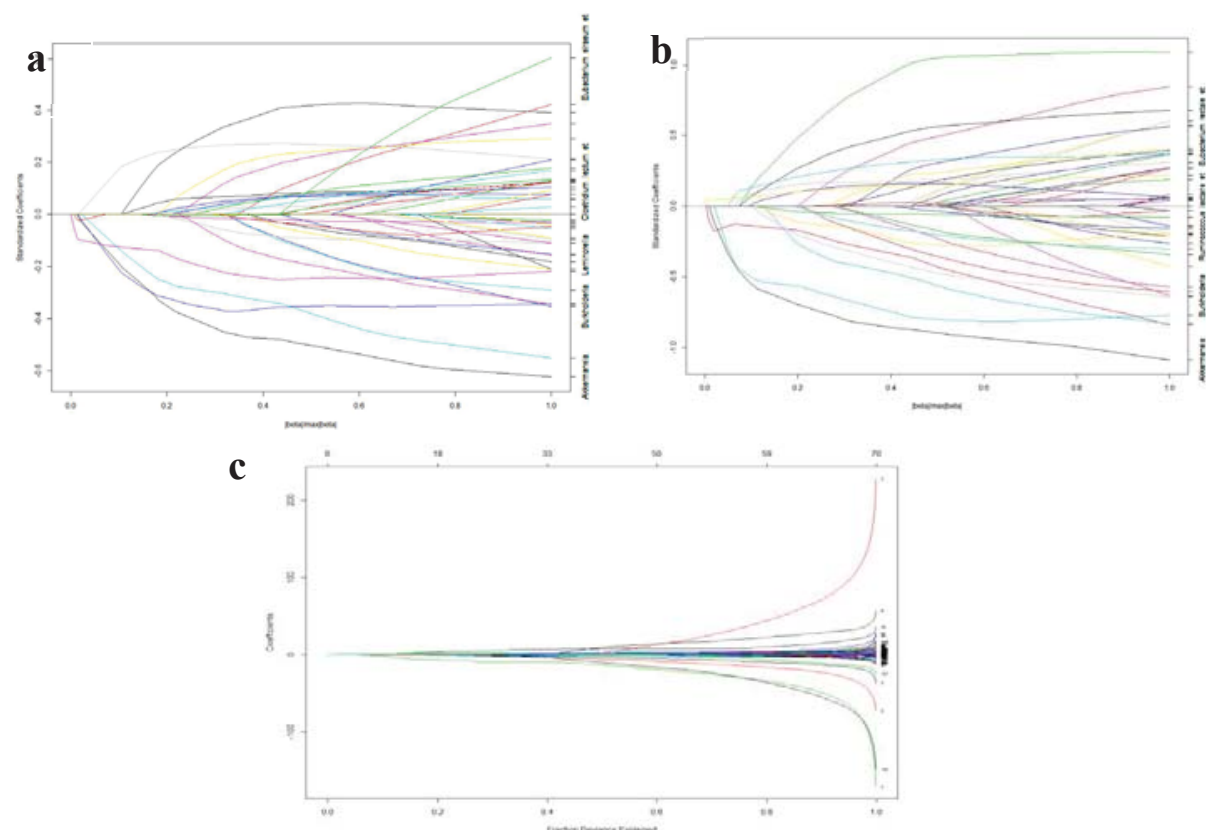

Supplementary Figure 2. Coefficient paths for the elastic net regression models applied to the gut microbiota data. (a) Elastic net regression estimates for BMI $z$-scores with the optimal lambda value $(\lambda=0.1)$ as obtained from ten-fold cross-validation parameters and $s=0.1$ on the horizontal axis. Selected bacterial groups with non-zero coefficients are the lines that fall below the cut-off value $\mathrm{s}=0.1 \quad(\mathrm{n}=5)$; (b) elastic net regression coefficients for weight $\mathrm{z}$-scores with tuning parameters $(\lambda=0.01$ and $\mathrm{s}=0.2)$ and non-zero bacterial group coefficients below $\mathrm{s}=0.2(\mathrm{n}=19)$; (c) elastic net regression coefficients for overweight with tuning parameters $(\lambda=0.01$ and $\mathrm{s}=0.1)$ with non-zero coefficients below $\mathrm{s}=0.1(\mathrm{n}=9)$
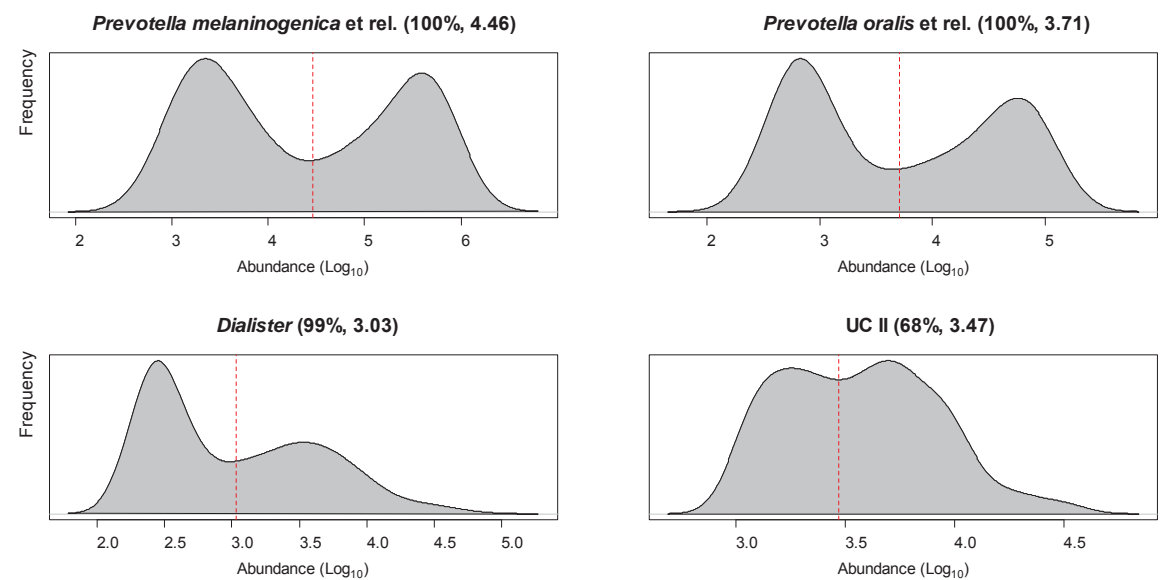

Supplementary Figure 3. Logarithmic abundance distributions of the four bacterial groups that exhibited a bimodal abundance across the gut microbiota of 295 children. High and low abundance states are separated by a dashed red line. Proportion of children with high levels of bimodal bacterial groups; $47.1 \%, 47.8 \%, 44.1 \%$ and $57.3 \%$ for Prevotella melanogenica et rel., Prevotella oralis et rel., Dialister and UCII respectively. UC II refers to the Uncultured Clostridiales II. 


\section{Chapter 4}

Effects of gut microbiota manipulation by antibiotics on host metabolism in obese humans: a randomized double-blind placebo-controlled trial

Dorien Reijnders*, Gijs H. Goossens*, Gerben D.A. Hermes, Evelien P.J.G. Neis, Christina M. van der Beek, Jasper Most, Jens J. Holst, Kaatje Lenaerts, Ruud S. Kootte, Max Nieuwdorp, Albert K. Groen, Steven W.M. Olde Damink, Mark V. Boekschoten, Hauke Smidt, Erwin G. Zoetendal, Cornelis H.C. Dejong and Ellen E. Blaak

*These authors contributed equally to this work 


\begin{abstract}
The gut microbiota has been implicated in obesity and cardiometabolic diseases, although evidence in humans is scarce. We investigated how gut microbiota manipulation by antibiotics (7-day administration of amoxicillin, vancomycin or placebo) affects host metabolism in 57 obese, prediabetic men. Vancomycin, but not amoxicillin, decreased bacterial diversity and reduced Firmicutes involved in short-chain fatty acid and bile acid metabolism, concomitant with altered plasma and/or fecal metabolite concentrations. Adipose tissue gene expression of oxidative pathways was upregulated by antibiotics, whereas immune-related pathways were downregulated by vancomycin. Antibiotics did not affect tissue-specific insulin sensitivity, energy/substrate metabolism, postprandial hormones and metabolites, systemic inflammation, gut permeability and adipocyte size. Importantly, energy harvest, adipocyte size and whole-body insulin sensitivity were not altered at 8-weeks follow-up, despite a still considerably altered microbial composition, indicating that interference with adult microbiota by 7-days antibiotic treatment has no clinically relevant impact on metabolic health in obese humans.
\end{abstract}




\section{Introduction}

Accumulating evidence indicates that the composition of the gut microbiota plays a prominent role in body weight regulation and the development of type 2 diabetes mellitus ${ }^{256,257}$. The gut microbiota regulates energy extraction from otherwise indigestible carbohydrates, determines the integrity of the intestinal epithelial layer, and influences the production and absorption of multiple signaling molecules involved in host metabolism. Several studies have demonstrated that germ-free mice are protected from diet-induced obesity, low-grade inflammation and glucose intolerance as compared to conventionally raised animals $^{48,103}$. Furthermore, it has been shown that transferring microbiota via fecal transplantation evoked alterations in body weight and insulin sensitivity in both rodents ${ }^{103}$ and humans ${ }^{258,259}$ Taken together, these data indicate that modulation of the gut microbiota may provide a promising avenue to target obesity-related metabolic disorders ${ }^{260}$.

The gut microbiota composition can be modulated by, amongst others, prebiotics, probiotics and antibiotics ${ }^{70}$, thereby altering the presence and expression of microbial genes and derived metabolites such as bile acids (BA) and short-chain fatty acids (SCFA) ${ }^{261,262}$. Particularly, the use of antibiotics has been associated with increased metabolic impairments, mainly when exposure occurs in early life ${ }^{263,264}$. Of note, these findings are primarily based on animal studies, in which the animals have mostly been exposed to a combination of antibiotics for periods varying from two to twenty weeks ${ }^{219,265-268}$. It has recently been shown that antibiotics may improve peripheral insulin sensitivity in a small number of obese subjects ${ }^{80}$. Nevertheless, the effects observed in the latter study were relatively minor and, importantly, the study was not placebo-controlled. Thus, wellcontrolled, large human studies examining the effects and underlying mechanisms of microbiota modulation on host metabolism are currently lacking.

Here, we report on a randomized, double-blind, placebo-controlled trial that was designed to investigate the effects of broad and narrow-spectrum antibiotic treatment for seven days on gut microbiota composition, tissue-specific insulin sensitivity, energy expenditure, substrate oxidation, fecal and plasma BA and SCFA concentrations, gut permeability, abdominal subcutaneous adipocyte size and systemic low-grade inflammation in obese men with impaired glucose homeostasis. Moreover, eight weeks after cessation of antibiotic treatment, we again determined microbiota composition, whole-body insulin sensitivity (HOMA-IR), fecal energy harvest and adipocyte size. 


\section{Methods}

\section{Study participants}

57 low active ( $<3 \mathrm{~h}$ organized sports activities per week), weight-stable $(<2 \mathrm{~kg}$ body weight change 3 months prior to inclusion) overweight/obese (BMI $25-35 \mathrm{~kg} / \mathrm{m}^{2}$ ), Caucasian men, between 35 and 70 years with impaired glucose metabolism (either fasting glucose $>6.1$ $\mathrm{mmol} / \mathrm{l}$, and/or $2 \mathrm{~h}$ glucose between $7.8-11 \mathrm{mmol} / \mathrm{l}$ ) and HOMA-IR $>2.2$ were included in this study (ClinicalTrials.gov, NCT02241421). Subjects were recruited via advertisements in local newspapers, and were all living in the area around Maastricht, The Netherlands. All subjects gave written informed consent for participation in this study, which was reviewed and approved by the local Medical Ethical Committee of Maastricht University Medical Center+. All procedures were according to the declaration of Helsinki (revised version, October 2008). Exclusion criteria were: the use of antibiotics for a period of three months before entering the study, known allergic reactions to any type of antibiotics, hearing disorders, cancer, liver malfunction, major illnesses with a life expectancy of less than five years and pulmonary, hepatic, cardiovascular, kidney, and gastrointestinal disease. Subjects did not use $\beta$-blockers, lipid and glucose lowering-drugs, anti-oxidants or chronic corticosteroids.

\section{Study design \& randomization}

This randomized, placebo-controlled, double-blind study had a three-armed parallel design. Participants were randomized to oral intake of amoxicillin (broad-spectrum antibiotic), vancomycin (directed against Gram-positive bacteria) or placebo (microcrystalline cellulose) for seven consecutive days $(1500 \mathrm{mg} / \mathrm{d})$. Antibiotics and placebo were equally capsulated to blind the content to subjects and investigators (BasicPharma, The Netherlands). The allocation sequence was established by computer-generated randomization (https://nl.tenalea.net). Blockrandomization with stratification for BMI, age and $2 \mathrm{~h}$-glucose values was used to increase the homogeneity of the treatment arms (block size, $n=6$ ). After completion of the study, returned capsules were counted to assess compliance. Participants were asked to maintain their habitual physical activity pattern and dietary habits (monitored by 3-day food diaries) throughout the study. The evening before an investigation day, a low fiber, low fat meal was consumed. Before and after intervention, study measurements were conducted following a 10-h overnight fast. To ensure complete systemic and gastrointestinal clearance of antibiotics, a 2-day wash-out period was taken into account before post-treatment measurements. Participants returned for a follow-up visit eight weeks after treatment cessation. 


\section{Hyperinsulinemic-euglycemic clamp}

A two-step hyperinsulinemic-euglycemic clamp combined with a $\left[6,6-{ }^{2} \mathrm{H}_{2}\right]$-glucose tracer (Cambridge Isotope Laboratories, Andover, MA, USA) was performed to measure Rd, $\mathrm{EGP}^{269}$ and the insulin-mediated suppression of $\mathrm{FFA}^{270,271}$. Blood samples were taken from a superficial dorsal hand vein, which was arterialized by using a hot-box $\left(\sim 50^{\circ} \mathrm{C}\right)$. After a bolus-injection $(2.4 \mathrm{mg} / \mathrm{kg})$, tracer-infusion was started at $0.04 \mathrm{mg} / \mathrm{kg} / \mathrm{min}$, which was continued throughout the measurement. After $2 \mathrm{~h}$, low-dose insulin was infused at 10 $\mathrm{mU} / \mathrm{m}^{2} / \mathrm{min}$ for $2 \mathrm{~h}^{272}$, followed by high-dose insulin at $40 \mathrm{mU} / \mathrm{m}^{2} / \mathrm{min}$ for $2 \mathrm{~h}^{273}$. By variable co-infusion of a $17.5 \%$-glucose solution, enriched by $1.1 \%$ tracer, plasma glucose concentrations were maintained at $5.0 \mathrm{mmol} / \mathrm{l}$. For calculation of steady-state-kinetics, additional blood samples were taken in the last $30 \mathrm{~min}$ of each step $(0,10$ and 40 $\mathrm{mU} / \mathrm{m}^{2} / \mathrm{min}$ insulin).

\section{Postprandial test}

Blood was sampled from a superficial dorsal hand vein, which was arterialized by placing the hand into a hot-box $\left(\sim 50^{\circ} \mathrm{C}\right)$. Blood samples were taken during the fasting state $(\mathrm{t}-30, \mathrm{t}-$ $15, \mathrm{t} 0 \mathrm{~min})$ and postprandial $(\mathrm{t}=30,60,90,120,180$ and $240 \mathrm{~min})$ after ingestion of the test meal. The liquid test meal, that was consumed within 5 min, provided $2.6 \mathrm{MJ}$ (61 E\% fat, $33 \mathrm{E} \%$ carbohydrate, $6 \mathrm{E} \%$ protein), which was consumed within 5 min at $\mathrm{t}=0^{274}$.

\section{Indirect calorimetry}

For indirect calorimetry during fasting $(30 \mathrm{~min}$ ) and the 4-h postprandial state, the opencircuit ventilated hood system was used (Omnical, Maastricht University, Maastricht, Netherlands $)^{275}$. Calculations of energy expenditure and substrate oxidation were performed according to the formulas of Weir ${ }^{276}$ and Frayn ${ }^{277}$. Nitrogen excretion was based on the assumption that protein oxidation represents $\sim 15 \%$ of total energy expenditure ${ }^{278}$.

\section{Gut Permeability test}

After baseline urine collection, subjects drank a $150 \mathrm{ml}$ multisaccharide test mix $[1 \mathrm{~g}$ sucrose (Van Gilse, Dinteloord, the Netherlands), $1 \mathrm{~g}$ lactulose (Centrafarm, Etten-Leur, the Netherlands), $1 \mathrm{~g}$ sucralose (Brenntag, Sittard, the Netherlands), $1 \mathrm{~g}$ erythritol (Danisco Sweeteners, Copenhagen, Denmark), $0.5 \mathrm{~g}$ of 1-rhamnose (Danisco) $]^{279}$. Urine was collected for determination of the urinary sucrose concentration in the 0-120 min urine collection, representing gastro-duodenal permeability, whereas in this collection small intestinal permeability is represented by the lactulose/rhamnose ratio. Proximal colon permeability is represented by the suclarose/erythritol ratio of the 120-300 $\mathrm{min}$ urine collection.

\section{Biochemical analyses for plasma variables}

Blood was collected into pre-chilled tubes, centrifuged at $1000 \mathrm{~g}$, and plasma was snapfrozen and stored at $-80^{\circ} \mathrm{C}$ until analyses. Isotopic enrichment of plasma glucose was determined by electron ionization gas chromatography-mass spectrometry and expressed as 
tracer-to-tracee ratio for steady-state calculations of Rd and EGP ${ }^{269}$. Plasma glucose, lactate, FFA and glycerol were determined with the Cobas Fara auto-analyzer (Roche, Switzerland). Plasma insulin was measured with a double antibody radioimmunoassay (Millipore, MA, USA). Plasma leptin concentrations were analyzed using commercially available radioimmunoassay kits (Human Leptin RIA, Millipore Corporation, Billerica, MA, USA). Plasma ANGPTL4 concentrations were measured by ELISA as described ${ }^{280}$. Plasma concentrations of IL- 6 , IL- 8 and TNF- $\alpha$ were determined using a multiplex enzyme-linked immuno-sorbent assay (Human ProInflammatory II 4-Plex Ultra-Sensitive Kit, Meso Scale Diagnositics, Rockville, MD, USA). Isocratic ion-exchange HPLC (Model PU-1980 pump, Jasco Easton, MD) with mass spectrometry (Model LTQ XL, Thermo Fisher Scientific, Waltham, MA) was used to determine sugar concentrations in plasma and urine for gastrointestinal permeability assessment ${ }^{279}$. LPS-binding protein was measured using non-commercial ELISA as described before ${ }^{281}$. Plasma concentrations of GLP-1 were measured by radioimmunoassays as previously described ${ }^{282}$. Plasma BA profile was measured using liquid chromatography tandem mass spectrometry (LC-MS/MS $)^{80}$. The total amount of primary (cholic acid and chenodeoxycholic acid and their taurine and glycine conjugated forms) and secondary BA (deoxycholic acid, lithocholic acid and their conjugated forms) was calculated as the sum of the individually quantified BA. Plasma SCFA were determined by LC-MS/MS as reported before ${ }^{283}$. The detection limits for acetate, propionate and butyrate were $0.1,0.05$ and $0.05 \mu \mathrm{mol} / \mathrm{L}$, respectively.

\section{Laboratory analysis of adipose tissue}

Abdominal subcutaneous AT biopsies were taken under local anesthesia under fasted conditions. One portion was embedded in paraffin. Sections were cut for staining, digital imaging and computerized morphometric measurement of individual adipocytes ${ }^{284}$. One portion $(\sim 500 \mathrm{mg})$ was snapfrozen in liquid nitrogen, from which RNA was extracted (Trizol chloroform extraction, Invitrogen, Cergy Pontoise, France) and used for microarray analysis. 100 ng total RNA was labeled by Whole-Transcript Sense Target Assay and hybridized to human whole-genome Affymetrix Gene 1.1 ST arrays, targeting 19793 unique genes (Affymetrix, Santa Clara, CA, USA). Quality control and data analysis pipeline have been described in detail previously ${ }^{285}$. Individual genes on the array were defined as changed when comparison of the normalized signal intensities showed a $\mathrm{FDRq}<0.05$ in a two-tailed paired t-test with Bayesan correction (Limma) ${ }^{286}$. Further functional data analysis was performed on the filtered data set with Gene Set Enrichment Analysis (GSEA, http://www.broad.mit.edu/gsea). Gene sets were selected based upon FDRq $<0.2$. Array data have been submitted to the Gene Expression Omnibus: number GSE76003.

\section{Laboratory analysis of feces}

Feces was collected at home for two consecutive days at baseline, seven days and eight weeks after intervention using the BMP commode specimen collection system, and divided 
over sterile tubes at home. Subjects were provided with a box of dry ice to freeze their stool samples immediately after defecation at approximately $-80^{\circ} \mathrm{C}$ and for transport to the university. Total fecal amount was weighed, and 24-h fecal samples were used to determine energy content using adiabatic bomb calorimetry (CBB 330, standard benzoic acid 6320 cal/g, BCS-CRMno90N). 24-h fecal BA composition was determined by using gas chromatography (GC) as described before ${ }^{269}$. Fecal SCFA were measured by gas chromatography-mass spectrometry (GC-MS, Medical laboratory 'Dr. Stein \& Colleague' Germany), according to the method described before ${ }^{287}$.

For microbiota profiling, DNA was isolated from 24-h fecal samples as described before ${ }^{80}$ and subsequently used for phylogenetic profiling using the HITchip phylogenetic microarray ${ }^{159}$. Standardized quality control was maintained through our library of a duplicated set of 3,631 probes targeting the 16S rRNA gene sequences of over 1,000 intestinal bacterial phylotypes. A more detailed description of microbiota profiling procedures can be found in the Supplemental Experimental Procedures.

\section{Statistics}

The calculated sample size ( $\mathrm{n}=19$ per treatment arm) was based on a $20 \%$ physiologically relevant change of insulin sensitivity $(\alpha=0.05, \beta=0.8)$. All data were evaluated for normality. Univariate analysis (ANOVA) was applied to compare group characteristics at baseline. Differences between treatments were analyzed using repeated-measures ANOVA with time and treatment as factors. ANCOVA analysis of the delta (post-pre value) was used for parameters when significantly different at baseline, taking the baseline value into account as covariate. The postprandial response (energy expenditure, substrate oxidation and GLP-1) is given as incremental area under the curve (iAUC/min), which was calculated by the trapezoid method. For HITchip analysis, $\log 10$-transformed signals were used as a proxy for bacterial logarithmic abundance. To determine which bacterial groups were significantly different in relative abundance before and after treatment within each group, a paired Wilcoxon test was used. Between-treatment group effects were assessed with linear mixed models using the lme4 package ${ }^{288}$. Benjamini-Hochberg correction was applied for multiple testing. We used Random Forests, a supervised machine-learning technique, and the pre and post treatment classes to confirm these results ${ }^{289}$. To determine whether individuals could be grouped into classes of specific metabolic responses to the interventions, we used the $1 \mathrm{cmm} \mathrm{R}$ package ${ }^{290}$ to perform Latent Class Analysis. Diversity of the microbiota was quantified based on non-logarithmized HITchip oligo-level signals by inverse Simpson's index using the Vegan package ${ }^{291}$. ANOVA with Tukey's Honest Significant post hoc analysis was applied to compare diversity between and within groups. Data are expressed as means \pm standard error of the mean (SEM), with a two-sided significance level of $\mathrm{P}<0.05$. Statistical analysis was performed using SPSS 20.0 for Macintosh and R 3.03. 


\section{Results and Discussion}

\section{Subject characteristics}

To study the role of the gut microbiota, we randomized 57 overweight and obese $35-70$ year old Caucasian men to oral administration of the broad-spectrum antibiotic amoxicillin (AMOX), narrow-spectrum antibiotic vancomycin (VANCO, directed against Grampositive bacteria), or placebo (PLA) for seven days. No significant differences in baseline characteristics were present between groups (Table 1). All subjects had impaired fasting glucose levels (plasma glucose $\geq 5.6 \mathrm{mmol} / \mathrm{l}$ ) and/or impaired glucose tolerance ( $2 \mathrm{~h}$ plasma glucose during a $75 \mathrm{~g}$ oral glucose tolerance test 7.8-11.1 mmol/1), and were insulin resistant (homeostasis model assessment for insulin resistance; HOMA-IR>2.2). One subject randomized to the AMOX intervention was considered a dropout due to use of other antibiotics during the study period. No serious adverse events and only a few cases of mild gastrointestinal discomfort were reported. There were no differences in daily energy and macronutrient intake, as monitored by a three-day food diary, between and within groups before and after intervention. Furthermore, body weight remained unchanged for all treatment groups throughout the study period and at follow-up (data not shown).

Table 2.1. Baseline characteristics of the study population

\begin{tabular}{|c|c|c|c|}
\hline Variable & PLA $(n=19)$ & $\operatorname{AMOX}(n=18)$ & VANCO $(n=19)$ \\
\hline Age (years) & $60.9 \pm 1.7$ & $55.7 \pm 1.5$ & $60.6 \pm 1.5$ \\
\hline Body weight (kg) & $96.7 \pm 2.3$ & $96.3 \pm 2.5$ & $97.6 \pm 1.9$ \\
\hline Body mass index $\left(\mathrm{kg} / \mathrm{m}^{2}\right)$ & $31.0 \pm 0.5$ & $31.1 \pm 0.8$ & $31.5 \pm 0.6$ \\
\hline Waist/Hip ratio & $1.04 \pm 0.01$ & $1.04 \pm 0.01$ & $1.07 \pm 0.01$ \\
\hline Waist circumference (cm) & $98.0 \pm 8.1$ & $101.1 \pm 6.4$ & $106.7 \pm 6.3$ \\
\hline Fasting glucose (mM) & $6.0 \pm 0.1$ & $6.1 \pm 0.1$ & $6.1 \pm 0.1$ \\
\hline 2h OGTT glucose (mmol/1) & $7.7 \pm 0.4$ & $7.0 \pm 0.5$ & $7.2 \pm 0.4$ \\
\hline Fasting insulin (mU/l) & $15.7 \pm 1.5$ & $17.9 \pm 1.6$ & $16.8 \pm 1.1$ \\
\hline HOMA-IR & $4.2 \pm 0.4$ & $4.9 \pm 0.5$ & $4.6 \pm 0.3$ \\
\hline $\mathrm{HbA}_{1 \mathrm{c}}(\%)$ & $5.5 \pm 0.1$ & $5.6 \pm 0.1$ & $5.6 \pm 0.1$ \\
\hline
\end{tabular}

Plus-minus values are means \pm SEM. HOMA-IR denotes homeostasis model assessment of insulin resistance, $\mathrm{HbA}_{1 \mathrm{c}}$ glycated haemoglobin

\section{Efficacy of microbiota manipulation by antibiotic treatment}

The fecal microbiota composition was determined by analyzing $16 \mathrm{~S}$ ribosomal RNA (rRNA) gene amplicons, using the Human Intestinal Tract Chip Microarray (HITChip) ${ }^{159}$, which showed that seven-day VANCO markedly decreased microbial diversity $(\mathrm{P}<0.001)$, whereas this was not affected by $\operatorname{AMOX}(\mathrm{P}=0.42)$ as compared to PLA (Figure 2.1). VANCO decreased the relative abundance of mainly Gram-positive bacteria of the 
Firmicutes phylum. Among the most strongly affected groups were genus-like groups that contain known butyrate-producing species from Clostridium clusters IV and XIVa, such as Coprococcus eutactus, Faecalibacterium prausnitzii and Anaerostipes caccae, as well as species involved in BA dehydroxylation such as Clostridium leptum. Conversely, Gramnegative Proteobacteria, members of Clostridium cluster IX and VANCO-resistant Grampositive bacilli such as Lactobacillus plantarum and Enterococcus, showed increased relative abundance after VANCO treatment (Figure 2.1 and Table S2.1), which is in line with previous studies ${ }^{80,292}$. This pattern was confirmed with a supervised machine-learning technique (Random Forests analysis, Table S2). Importantly, microbiota composition was still affected eight weeks after cessation of VANCO treatment. Microbial diversity was still lower $(\mathrm{q}=0.053)$, and overall similarity and composition were deviant from baseline (pretreatment) as compared to PLA. Although the bacterial groups that increased in abundance due to VANCO treatment had in general returned to baseline levels, several members of Clostridium clusters IV and XIVa were still decreased as compared to PLA. Furthermore, observed dynamics with respect to gut microbiota composition and diversity were individual-specific (Figure S2.1). In contrast, AMOX treatment did not affect microbiota composition after seven days treatment or at eight weeks follow-up compared to PLA, which is in accordance with a previous study in obese humans ${ }^{80}$. 


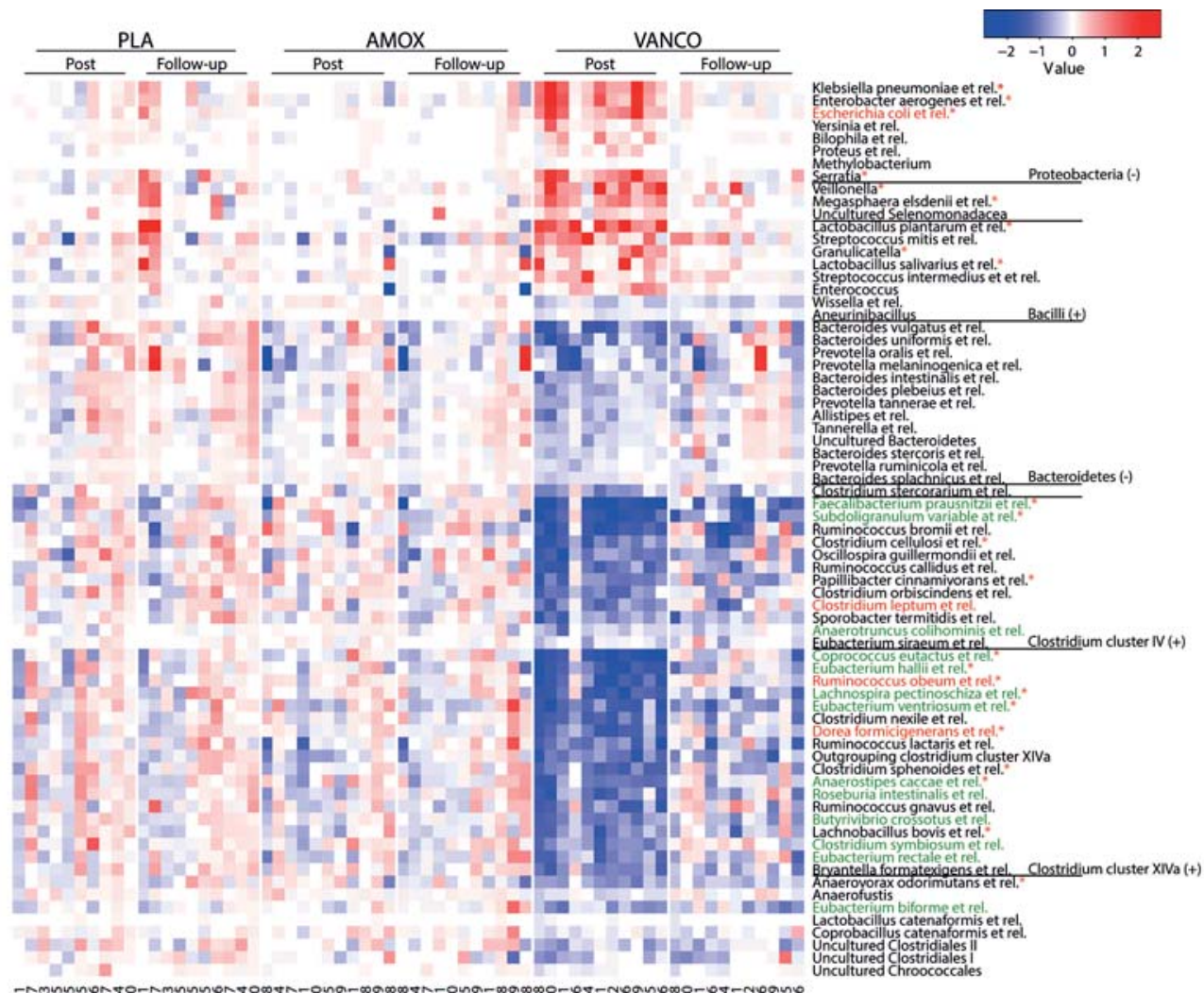

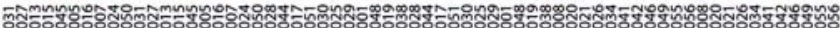

Figure 2.1. The effect of vancomycin and amoxicillin treatment on microbiota composition. Heatmap of bacterial groups (at genus and order like level with Gram staining between brackets) whose relative abundance was significantly different $(q<0.05)$ post-treatment within the VANCO group. Color value shows $\log 10$ fold changes compared to baseline. Genus like groups containing known butyrate producing and BA dehydroxylating species are depicted in green and red, respectively. *Groups that exhibited a significant difference between VANCO and PLA treatments.

\section{Antibiotic treatment does not affect tissue-specific insulin sensitivity}

The primary outcome of this study was peripheral insulin sensitivity (insulin-stimulated glucose rate of disappearance, Rd), as determined by a two-step hyperinsulinemiceuglycemic clamp with $\left[6,6{ }^{2}{ }^{2} \mathrm{H}_{2}\right]$-glucose tracer infusion. Antibiotic treatment did not significantly alter Rd as compared to PLA (Figure 2.2). Additionally, no effects were found on hepatic and adipose tissue (AT) insulin sensitivity, as determined by the insulinmediated suppression of endogenous glucose production (EGP) and plasma free fatty acid (FFA) concentrations, respectively. In accordance, antibiotic treatment did neither alter whole-body insulin sensitivity (HOMA-IR) immediately after cessation of treatment, nor at eight weeks follow-up (Figure S2). Our data are in contrast with several previous studies in rodents, which indicated that antibiotic treatment may improve glucose homeostasis and metabolic impairments ${ }^{265-268,}{ }^{293-295}$. Nevertheless, a more recent study showed that 
VANCO-treated mice had little weight change and no improvement in glycemic control ${ }^{296}$. Consistent with the present data, a four-day treatment with a broad-spectrum antibiotic cocktail did not affect postprandial glucose metabolism in lean healthy men ${ }^{297}$. Furthermore, it has recently been shown in a limited number of obese subjects with the metabolic syndrome that VANCO slightly but significantly reduced peripheral insulin sensitivity, despite comparable changes in microbial composition and BA metabolism as found in the present study ${ }^{80}$. Although the data of the latter study seems at odds with the present findings, it is important to emphasize that in the study by Vrieze and colleagues ${ }^{80}$ the modest $(\sim 4 \%)$ VANCO-induced decrease in peripheral insulin sensitivity was based on a within-group comparison (post-treatment versus pre-treatment), since a placebo group was not included in the study design. Additionally, in the present study, follow-up measurements that were performed eight weeks after treatment cessation also did not show an effect on whole-body insulin sensitivity, despite a still considerably altered microbial composition as compared to pre-treatment as well as placebo.

\section{Antibiotic treatment does not affect energy and substrate metabolism}

To examine the effect of gut microbiota modulation on postprandial metabolite concentrations, energy expenditure and substrate oxidation, we performed a high-fat mixedmeal test $(2.6 \mathrm{MJ}[61 \mathrm{E} \%$ fat, $33 \mathrm{E} \%$ carbohydrates, $6 \mathrm{E} \%$ protein]). We determined arterialized plasma metabolite concentrations and measured energy expenditure and substrate oxidation by whole-body indirect calorimetry. Neither VANCO nor AMOX significantly affected basal and postprandial plasma glucose, insulin, FFA, triacylglycerol (TAG) and lactate concentrations (Table 2.2 and Figure S2.3). Also, no significant effects on basal and postprandial energy expenditure, carbohydrate and fat oxidation were found (Figure 2.3). After adjustment for fecal weight, intestinal energy harvest, which is reflected by daily fecal energy content, was neither changed immediately after treatment cessation, nor after eight weeks follow-up (Figure 2.3). Although previous studies in rodents have shown a prominent role of the gut microbiota in energy harvest and body weight ${ }^{48,219}$, our findings suggest that antibiotics do not alter energy harvest in humans. Of note, in rodent studies, animals were exposed to antibiotics in their drinking water for two up to twenty weeks ${ }^{219}, 265-268,293-295$. Similarly, more prolonged treatment (four to six weeks) with a higher dosage or a combination of different antibiotics increased body weight in endocarditis patients ${ }^{298,299}$. These studies may indicate that a long-term dysbalance in microbiota composition has more pronounced effects as compared to short-term manipulation. However, it is hard to differentiate between the role of the gut microbiota and systemic effects of antibiotics in the latter studies. Noteworthy, we have applied a two-day wash-out period before post-treatment measurements were performed to exclude that effects may be mediated via direct systemic effects of antibiotics. Additionally, VANCO does not pass the gastrointestinal barrier and, therefore, does not reach the circulation ${ }^{300}$. 
A

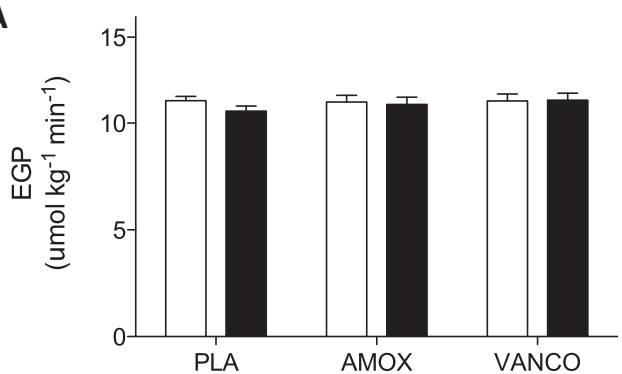

C

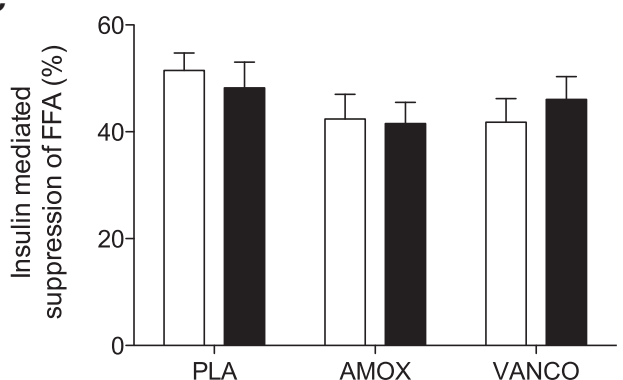

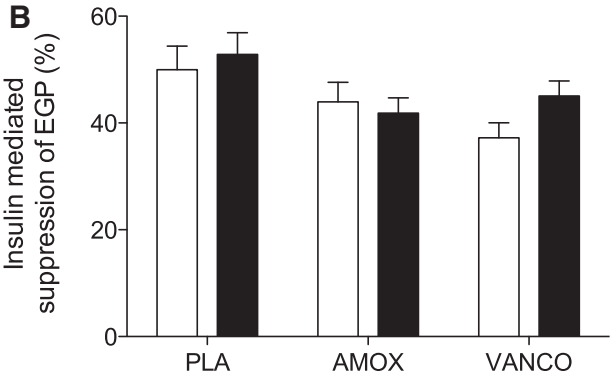

D

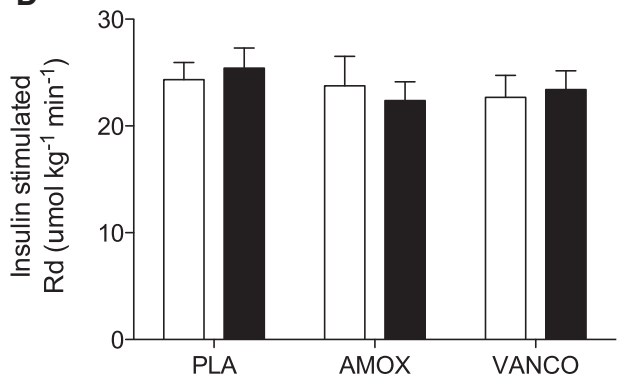

Figure 2.2. The effect of 7 days placebo, amoxicillin and vancomycin on hepatic, adipose tissue and peripheral insulin sensitivity. Bars represent means \pm SEM $(n=56)$. Tissue specific insulin sensitivity did not change by short-term antibiotics. (A) EGP: liver endogenous glucose production at baseline (B) steady state insulin-mediated EGP suppression (\%) upon $10 \mathrm{mU} / \mathrm{m} 2 / \mathrm{min}$ insulin infusion (C) steady state $10 \mathrm{mU} / \mathrm{m} 2 / \mathrm{min}$ insulin-mediated suppression (\%) of circulating free fatty acids (FFA) as measure for adipose tissue insulin sensitivity. (D) $40 \mathrm{mU} / \mathrm{m} 2 / \mathrm{min}$ insulin stimulated (oxidative) glucose disposal (Rd).

Antibiotic treatment does not alter gut permeability and systemic inflammatory markers

We investigated the effect of seven days of AMOX and VANCO treatment on gut permeability and the related translocation of bacterial lipopolysaccharide (LPS) from the intestinal lumen into the circulation. The pronounced VANCO-induced microbial alterations were not accompanied by changes in small intestine and proximal colon permeability (Figure S2.4), as assessed by a multi-saccharide test ${ }^{279}$. This is in accordance with unchanged LPS-binding protein (LBP) concentrations after VANCO and AMOX treatment as compared to PLA (Table 2.2). LPS, which is released by Gram-negative bacteria, may trigger the immune system by increasing inflammatory cytokine production in AT and is frequently used as an indicator of metabolic endotoxemia ${ }^{220}$. Therefore, we have additionally determined plasma interleukin (IL)-6, IL-8 and tumor necrosis factor (TNF)- $\alpha$ concentrations. In line with unchanged LBP concentrations, neither of these inflammatory factors was affected by seven-day VANCO or AMOX as compared to PLA. This was observed despite a substantial increase in relative abundance of potentially proinflammatory Gram-negative Proteobacteria. 

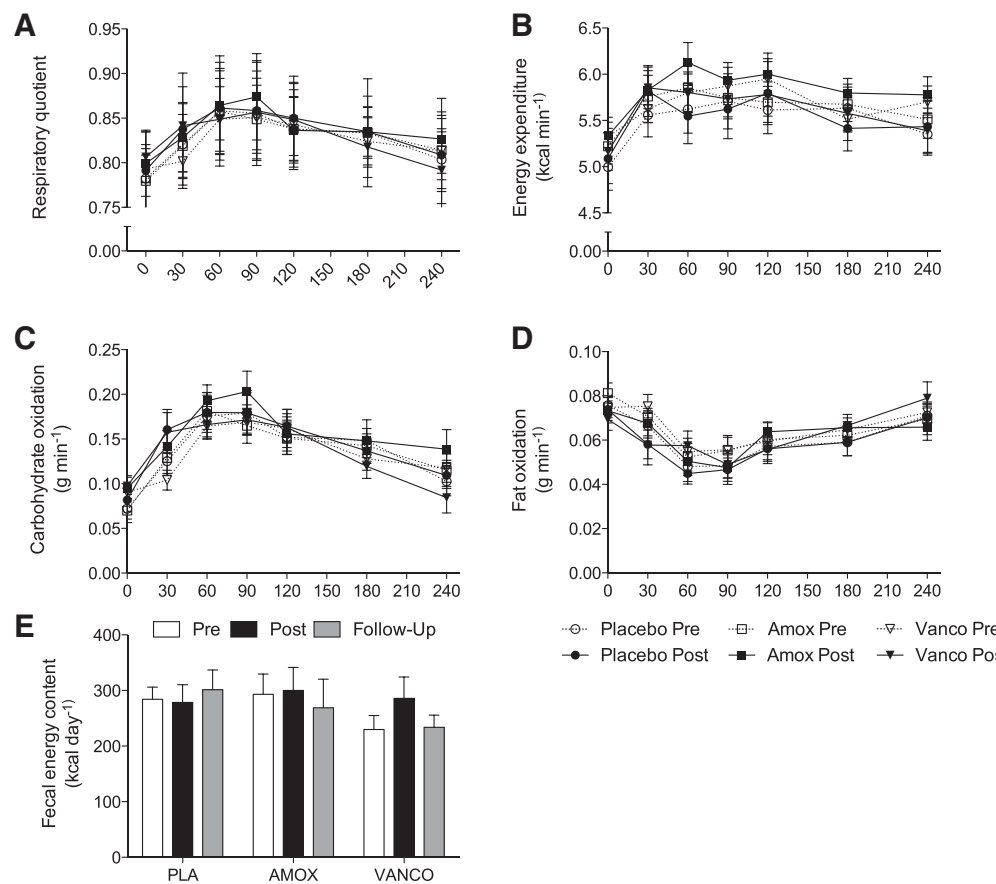

$\rightarrow$ Placebo Post $\rightarrow$ AmoxPost $\rightarrow$ Vanco Pos

Figure 2.3. The effect of 7 days placebo, amoxicillin and vancomycin on energy expenditure, substrate metabolism and fecal energy excretion. Data are given as means \pm SEM. Indirect calorimetry was performed during fasting conditions and for 4 hours after intake of a liquid high fat mixed meal (HFMM) in a subgroup of $n=37$. Mean O2-consumption and CO2production over 20 minutes were used for calculations. (A-D) Fasting respiratory quotient (RQ), energy expenditure (EE), carbohydrate oxidation and fat oxidation did not differ after intervention (time $\times$ treat $\mathrm{P}$ value $>0.05$ ). Incremental AUC's after ingestion of HFMM were also not affected by AMOX or VANCO. (E) Fecal Energy excretion (kcal/day) did not significantly change after VANCO or AMOX compared to PLA $(n=56)$.

\section{Vancomycin inhibits bile acid conversion and short-chain fatty acid production}

SCFA, notably butyrate, can be produced by several groups within the Firmicutes phylum (mainly Clostridium clusters XIVa and IV including Coprococcus eutactus and F. prausnitzii), some of which are also involved in BA dehydroxylation ${ }^{261,301}$. Indeed, we found a decreased relative abundance of these groups after VANCO, which was accompanied by a marked reduction in plasma $(\mathrm{P}=0.005)$ and fecal $(\mathrm{P}=0.001)$ concentrations of secondary BA as compared to PLA (Figure 2.4). This was accompanied by an increase of fecal primary $\mathrm{BA}(\mathrm{P}=0.013)$. In addition, fecal SCFA concentrations (acetate $(\mathrm{P}=0.001)$, butyrate $(\mathrm{P}<0.001)$, caproate $(\mathrm{P}<0.001)$ and valerate $(\mathrm{P}=0.009)$ were significantly decreased following $\mathrm{VANCO}$, whilst in plasma only butyrate tended to decrease after VANCO $(\mathrm{P}=0.078)$ but not following AMOX treatment (Figure 2.5). Although BA and SCFA may control incretin release $\mathrm{s}^{262,302}$ and affect energy metabolism in rodents ${ }^{303}$, no effects on postprandial energy and substrate metabolism, and fasting and postprandial glucagon-like peptide 1 (GLP-1) concentrations were found in the present study (Table 2.2). 
Table 2.2. Effect of 7 days placebo, amoxicillin and vancomycin on metabolic, inflammatory, and hormonal parameters

\begin{tabular}{|c|c|c|c|c|c|}
\hline Variable & & $\operatorname{PLA}(\mathrm{N}=14)$ & $\operatorname{AMOX}(\mathrm{N}=12)$ & $\operatorname{VANCO}(\mathrm{N}=12)$ & P value ${ }^{a}$ \\
\hline \multicolumn{6}{|c|}{ Fasting plasma metabolite and ANGPTL4 concentrations } \\
\hline \multirow[t]{2}{*}{ Glucose (mM) } & Pre & $6.31 \pm 1.12$ & $6.48 \pm 0.25$ & $6.25 \pm 0.19$ & $0.177^{\mathrm{b}}$ \\
\hline & Post & $6.29 \pm 0.14$ & $6.39 \pm 0.20$ & $5.99 \pm 0.13$ & \\
\hline \multirow[t]{2}{*}{ TAG $(\mathrm{mM})$} & Pre & $1.40 \pm 0.17$ & $1.08 \pm 0.15$ & $1.03 \pm 0.09$ & 0.511 \\
\hline & Post & $1.47 \pm 0.21$ & $1.04 \pm 0.15$ & $1.06 \pm 0.10$ & \\
\hline \multirow[t]{2}{*}{ FFA (uM) } & Pre & $699 \pm 34$ & $683 \pm 48$ & $679 \pm 38$ & $0.423^{\mathrm{b}}$ \\
\hline & Post & $661 \pm 34$ & $579 \pm 58$ & $626 \pm 54$ & \\
\hline \multirow[t]{2}{*}{ Lactate (mM) } & Pre & $0.80 \pm 0.07$ & $0.93 \pm 0.04$ & $0.88 \pm 0.11$ & 0.238 \\
\hline & Post & $0.91 \pm 0.11$ & $0.90 \pm 0.05$ & $0.79 \pm 0.06$ & \\
\hline \multirow[t]{2}{*}{ ANGPTL4 (ng/ml) } & Pre & $5.1 \pm 0.7$ & $4.3 \pm 0.5$ & $4.9 \pm 0.5$ & 0.137 \\
\hline & Post & $5.5 \pm 0.7$ & $3.8 \pm 0.5$ & $4.3 \pm 0.3$ & \\
\hline \multicolumn{6}{|c|}{ Postprandial (0-4h) plasma metabolite concentrations } \\
\hline \multirow[t]{2}{*}{ Glucose (iAUC/min) } & Pre & $0.60 \pm 0.10$ & $0.50 \pm 0.12$ & $0.41 \pm 0.19$ & 0.633 \\
\hline & Post & $0.54 \pm 0.13$ & $0.55 \pm 0.10$ & $0.45 \pm 0.09$ & \\
\hline \multirow[t]{2}{*}{ TAG (iAUC/min) } & Pre & $0.84 \pm 0.07$ & $0.83 \pm 0.10$ & $0.75 \pm 0.10$ & $0.945^{\mathrm{b}}$ \\
\hline & Post & $0.92 \pm 0.07$ & $0.94 \pm 0.01$ & $0.81 \pm 0.07$ & \\
\hline \multirow[t]{2}{*}{ FFA } & Pre & $-364 \pm 27$ & $-341 \pm 36$ & $-339 \pm 31$ & $0.547^{\mathrm{b}}$ \\
\hline & Post & $-332 \pm 28$ & $-245 \pm 37$ & $-300 \pm 40$ & \\
\hline \multirow{2}{*}{$\begin{array}{l}\text { Lactate } \\
(\mathrm{iAUC} / \mathrm{i})\end{array}$} & Pre & $0.65 \pm 0.05$ & $0.45 \pm 0.07$ & $0.40 \pm 0.08$ & $0.154^{\mathrm{b}}$ \\
\hline & Post & $0.50 \pm 0.05$ & $0.41 \pm 0.06$ & $0.41 \pm 0.06$ & \\
\hline \multicolumn{6}{|c|}{ Fasting plasma hormone concentrations } \\
\hline \multirow[t]{2}{*}{ GLP-1 (pmol/1) } & Pre & $8.7 \pm 0.7$ & $8.5 \pm 0.7$ & $9.7 \pm 1.1$ & 0.670 \\
\hline & Post & $9.3 \pm 1.1$ & $8.7 \pm 0.8$ & $10.2 \pm 1.2$ & \\
\hline \multirow[t]{2}{*}{ Insulin $(\mathrm{mU} / \mathrm{l})$} & Pre & $11.5 \pm 1.3$ & $12.6 \pm 1.3$ & $14.3 \pm 1.8$ & 0.504 \\
\hline & Post & $12.7 \pm 1.6$ & $13.4 \pm 1.8$ & $13.9 \pm 1.5$ & \\
\hline \multirow[t]{2}{*}{ Leptin (ng/ml) } & Pre & $11.4 \pm 1.6$ & $10.1 \pm 2.1$ & $9.7 \pm 0.8$ & $0.106^{\mathrm{b}}$ \\
\hline & Post & $12.9 \pm 2.3$ & $10.0 \pm 1.8$ & $8.8 \pm 0.8$ & \\
\hline \multicolumn{6}{|c|}{ Postprandial $(0-4 \mathrm{~h})$ plasma hormone concentrations } \\
\hline \multirow[t]{2}{*}{ GLP-1 (iAUC/min) } & Pre & $5.0 \pm 0.7$ & $4.3 \pm 0.7$ & $3.4 \pm 0.6$ & 0.451 \\
\hline & Post & $4.5 \pm 0.8$ & $4.3 \pm 0.8$ & $4.3 \pm 0.9$ & \\
\hline \multirow{2}{*}{$\begin{array}{l}\text { Insulin } \\
(\mathrm{iAUC} / \mathrm{i})\end{array}$} & Pre & $21.1 \pm 2.1$ & $20.0 \pm 3.0$ & $25.0 \pm 4.5$ & 0.294 \\
\hline & Post & $23.1 \pm 3.3$ & $21.7 \pm 3.0$ & $22.5 \pm 3.0$ & \\
\hline \multicolumn{6}{|c|}{ Fasting inflammatory marker concentrations } \\
\hline \multirow[t]{2}{*}{ LBP (pg/ml) } & Pre & $19.6 \pm 1.8$ & $17.5 \pm 1.8$ & $25.7 \pm 4.3$ & 0.456 \\
\hline & Post & $18.4 \pm 3.3$ & $20.4 \pm 2.9$ & $23.6 \pm 3.6$ & \\
\hline \multirow[t]{2}{*}{ IL-6 (pg/ml) } & Pre & $0.8 \pm 0.1$ & $0.8 \pm 0.1$ & $1.0 \pm 0.1$ & 0.775 \\
\hline & Post & $1.0 \pm 0.1$ & $0.8 \pm 0.1$ & $1.1 \pm 0.1$ & \\
\hline \multirow[t]{2}{*}{ IL-8 (pg/ml) } & Pre & $6.2 \pm 0.5$ & $4.3 \pm 0.4^{\mathrm{c}}$ & $5.2 \pm 0.4$ & 0.444 \\
\hline & Post & $5.9 \pm 0.5$ & $4.8 \pm 0.4$ & $5.9 \pm 0.4$ & \\
\hline \multirow[t]{2}{*}{ TNF- $\alpha(\mathrm{pg} / \mathrm{ml})$} & Pre & $2.6 \pm 0.1$ & $2.3 \pm 0.1^{\mathrm{c}}$ & $2.7 \pm 0.1$ & 0.424 \\
\hline & Post & $2.7 \pm 0.1$ & $2.5 \pm 0.1$ & $2.8 \pm 0.1$ & \\
\hline
\end{tabular}

Data are mean \pm SEM. For determination of plasma hormones and metabolites, only a subgroup of $n=38$ was analyzed. There were no significant differences between the groups after intervention (Post) compared to baseline (Pre). Triacylglycerol (TAG), free fatty acids (FFA), glucagon-like peptide (GLP), angiopoietin-like 4 (ANGPTL4), lipopolysaccharide-binding protein (LBP), interleukin (IL), tumor necrosis factor (TNF). ${ }^{\text {a }} \mathrm{P}$ value represents the overall intervention effect between groups assessed by repeated measures ANOVA (time $\times$ treat $\mathrm{P}$ value) or ANCOVA when baseline concentrations were different between groups. ${ }^{\mathrm{b}}$ time effect $(\mathrm{P}<0.05)$. ${ }^{\mathrm{c}}$ baseline group difference $(\mathrm{P}<0.05)$ 


\section{Antibiotic treatment alters adipose tissue gene expression but not adipocyte morphology.}

To determine the effect of an altered gut microbiota composition on AT, we collected abdominal subcutaneous AT biopsies to examine adipocyte size and gene expression profiles using Affymetrix microarray transcriptomic analysis. Antibiotic treatment had no significant effect on abdominal subcutaneous adipocyte size and the proportion of small and large adipocytes, neither directly after treatment cessation nor at eight weeks follow-up (Figure S2.5). Remarkably, when comparing the gene expression data with the Kyoto Encyclopedia of Genes and Genomes (KEGG), we found that VANCO and, to a lesser extent, AMOX increased AT expression of genes involved in pathways related to peroxisome-proliferator activated receptor(PPAR)-signaling and of genes encoding proteins involved in the mitochondrial Krebs cycle, fatty acid degradation and other components of the oxidative machinery, suggestive of increased oxidative metabolism in AT (Figure S2.6). In addition, VANCO decreased the expression of histone clustering genes. Although we found no differences in adipocyte morphology and circulating FFA, TAG, leptin and angiopoietin-like 4 (ANGPTL4) concentrations (Table 2.2), these alterations in the AT transcriptome may translate into changes in AT function over longer periods of time.

Finally, VANCO decreased the expression of gene sets involved in apoptosis and nuclear factor NFKB signaling as well as adaptive and innate immune responses, including genes of major histocompatibility complex-I, T-cell, B-cell and Natural Killer cell signaling. In contrast, genes related to lysosomal breakdown were upregulated as compared to PLA (Table S2.3). Lower NFKB-dependent gene expression and diminished NK and CD8+ T cell function in macrophages have been observed in germ-free and antibiotic-treated mice ${ }^{304}$. In the latter study, the effects were ascribed to a reduced activation of Farnesoid $\mathrm{X}$ receptors by a reduction of unconjugated and secondary $\mathrm{BA}^{261}$, which seems in line with the present findings. In addition, although the exact role of SCFA in the systemic and AT immune cell responses is unknown, SCFA may be involved in the regulation of T-cells in the gut and peripheral tissues via the G-protein coupled receptor $43^{262,305-307}$. Despite the effects of antibiotic treatment on the KEGG-pathways described above, no significant associations $(\mathrm{FDR}<0.25)$ were found between individual bacterial groups and AT gene expression (data not shown). 


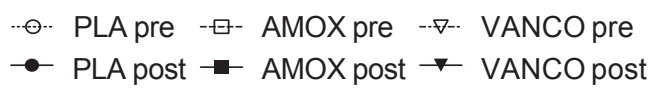

A

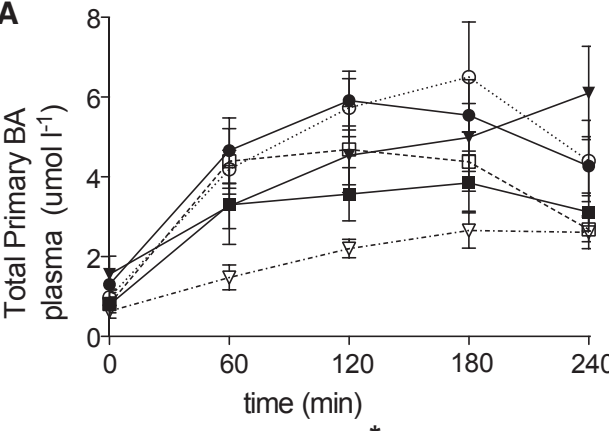

C

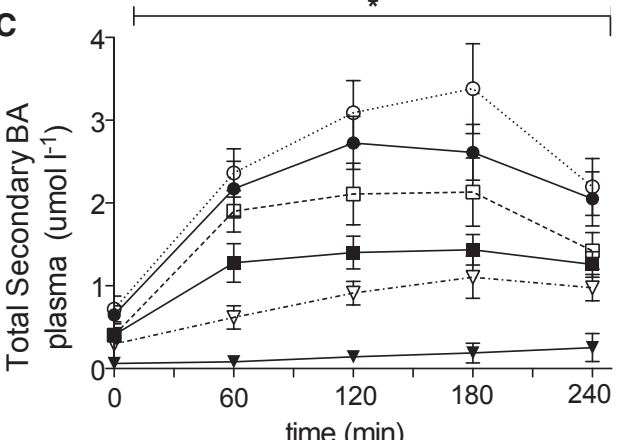

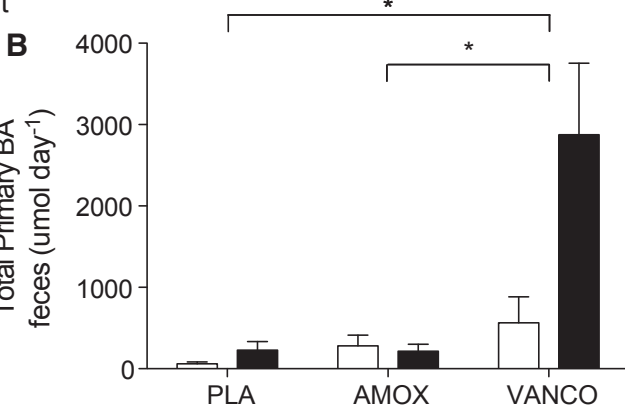
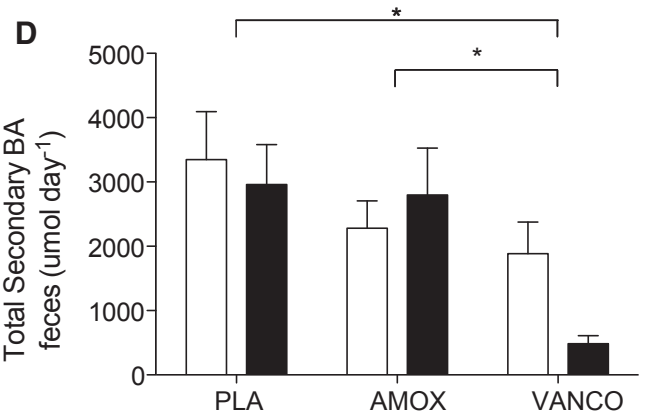

Figure 2.4. The effect of 7 days placebo, amoxicillin and vancomycin on bile acid concentrations in plasma and feces. Means \pm SEM. In a subgroup of $n=37$, (A) plasma postprandial primary BA did not change significantly, (B) VANCO increased fecal primary BA, (C) decreased plasma secondary BA and (D) fecal secondary BA compared to PLA and AMOX. * time $\times$ treat P-value $<0.05$ for VANCO vs. PLA.

\section{Microbial groups are not associated with host metabolic parameters}

Although overall host metabolism did not change significantly following antibiotic treatment, we used univariate and multivariate statistics (redundancy analysis) to assess possible associations between specific characteristics of gut microbial profiles and host metabolic parameters. However, we did not find any significant and consistent associations when we evaluated the abundance and dynamics of individual bacterial taxa, combinations of taxa, the complete microbiota and bacterial diversity at baseline, as well as seven days and eight weeks post-intervention.

Furthermore, we investigated whether we could identify and connect patterns of specific metabolic and/or microbiological perturbations with the response to the intervention. First, we evaluated the stratification of subjects based on the extent of the microbial shift in diversity, as well as microbial composition. Secondly, based on the extent and direction of the metabolic response to the intervention, we used univariate and cluster analysis to discover microbial patterns. Lastly, we used latent class analysis ${ }^{308}$, to define groups of subjects with certain metabolic patterns before and after treatment. Neither of these 
analyses showed groups of individuals with specific associations of the microbiota with host metabolic parameters (data not shown).

Pre

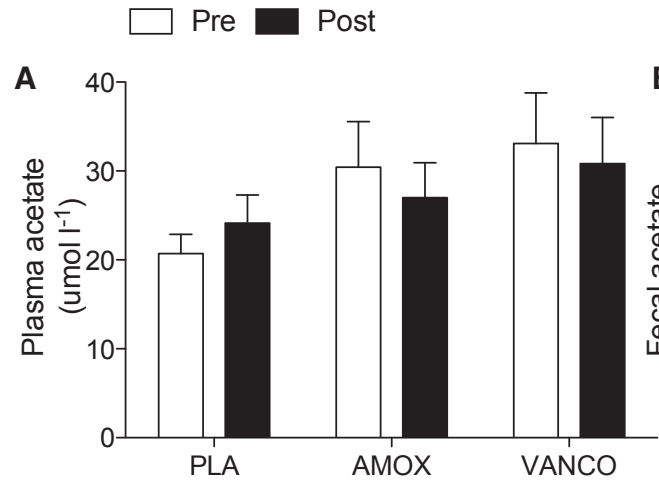

$\square$ Pre $\square$ Post $\square$ Follow-up

B

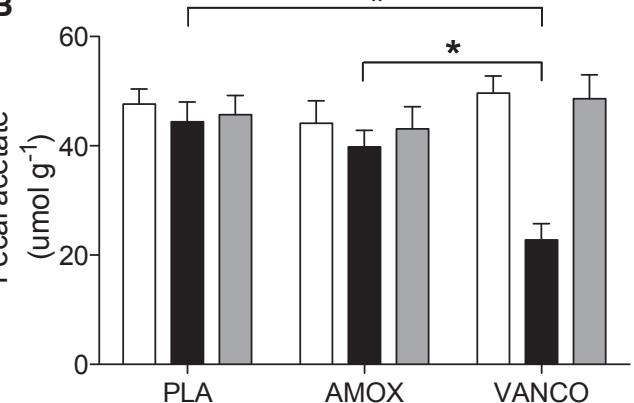

D

C
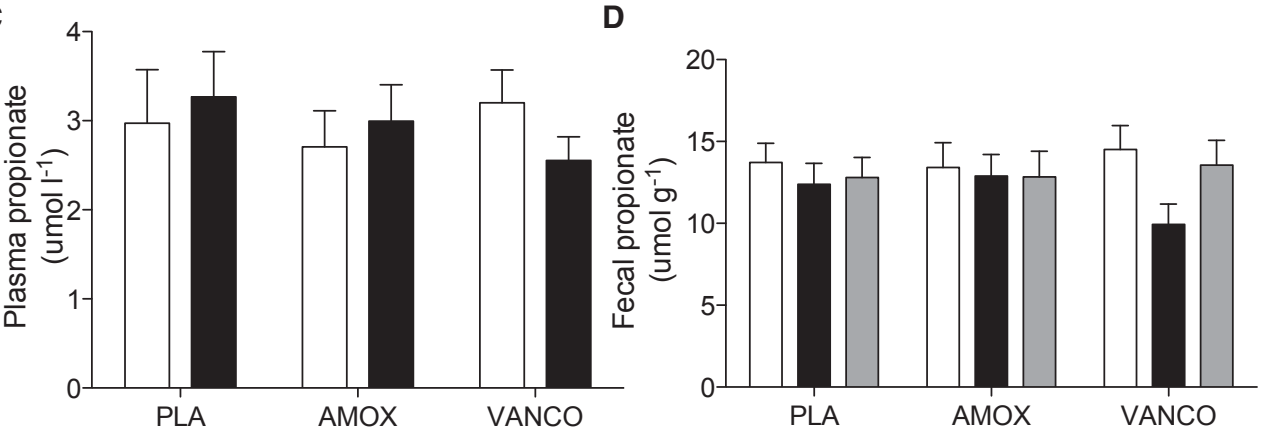
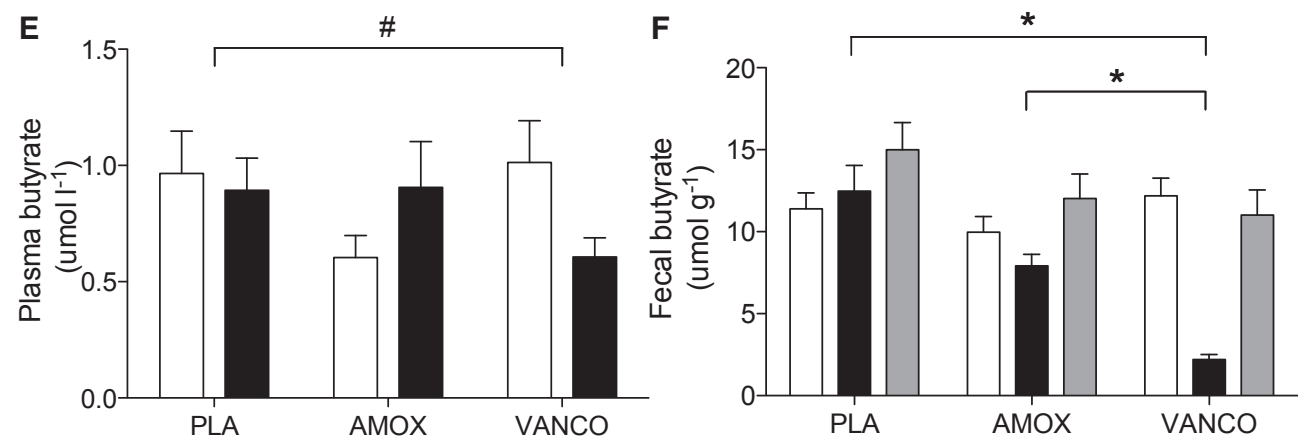

Figure 2.5: The effect of 7 days placebo, amoxicillin and vancomycin on plasma and fecal short chain fatty acid concentrations. Values are given as mean \pm SEM $(n=56)$. No significant effect was found for (A) plasma acetate, (C) plasma propionate, $(\mathrm{D})$ fecal propionate, and a trend $(\# \mathrm{P}=0.07)$ for $(\mathrm{E})$ plasma butyrate. Fecal acetate and butyrate $(\mathrm{B}, \mathrm{F})$ decreased after VANCO treatment but not after AMOX. * time $\times$ treat P-value $<0.05$ for VANCO vs. PLA and vs. AMOX 


\section{Perspectives}

In the present study, we demonstrated that seven days VANCO treatment markedly affected microbial diversity and composition, which was accompanied by a reduced conversion of primary to secondary BA and a lower production of SCFA in the gut. Importantly, these alterations did not translate into significant effects on peripheral, hepatic and AT insulin sensitivity, energy and substrate metabolism and systemic low-grade inflammation immediately after treatment cessation. Moreover, no clinically relevant effects on energy harvest, abdominal subcutaneous adipocyte size and whole-body insulin sensitivity (HOMA-IR) were found at eight weeks follow-up. In contrast to VANCO, no effects of AMOX treatment on gut microbial composition, metabolic and inflammatory parameters were found. Taken together, the present study implies that interference with a resilient adult microbiota by antibiotics has no clinically relevant short-term (seven days) and long-term (eight weeks) effects on the metabolic parameters measured in this study. This contradicts many previous rodent studies and again highlights that rodent data cannot always be extrapolated to humans.

Noteworthy, several nuances have to be made with respect to the conclusions of the present study. First, since we studied obese, insulin resistant men with impaired glucose metabolism, we cannot exclude that microbiota manipulation by antibiotics may have more pronounced effects in women or less metabolically compromised individuals. Secondly, the duration of the intervention was relatively short, compared to rodent studies. Furthermore, it has been demonstrated that the risk of developing type 2 diabetes was increased when subjects were exposed to $>5$ antibiotic treatments ${ }^{309}$, and that the number of prescriptions may accelerate the ageing-related decline of intestinal integrity $^{310}$. Of note, the participants that were included in the present study had received on average 1.7 antibiotic treatments over the past 10 years, without any antibiotic use 3 months prior to the start of the study. As mentioned above, several studies have indicated that a long-term or more frequent perturbation in microbiota composition may have more pronounced effects on metabolic health than short-term manipulation. For this reason, it is important to emphasize that the present study does not exclude an important role for the gut microbiota manipulations in changes of host metabolism. This should be further investigated in future prospective and long-term (dietary, prebiotic and/ or probiotic) intervention studies in humans. 


\section{Supplementary figures and tables}
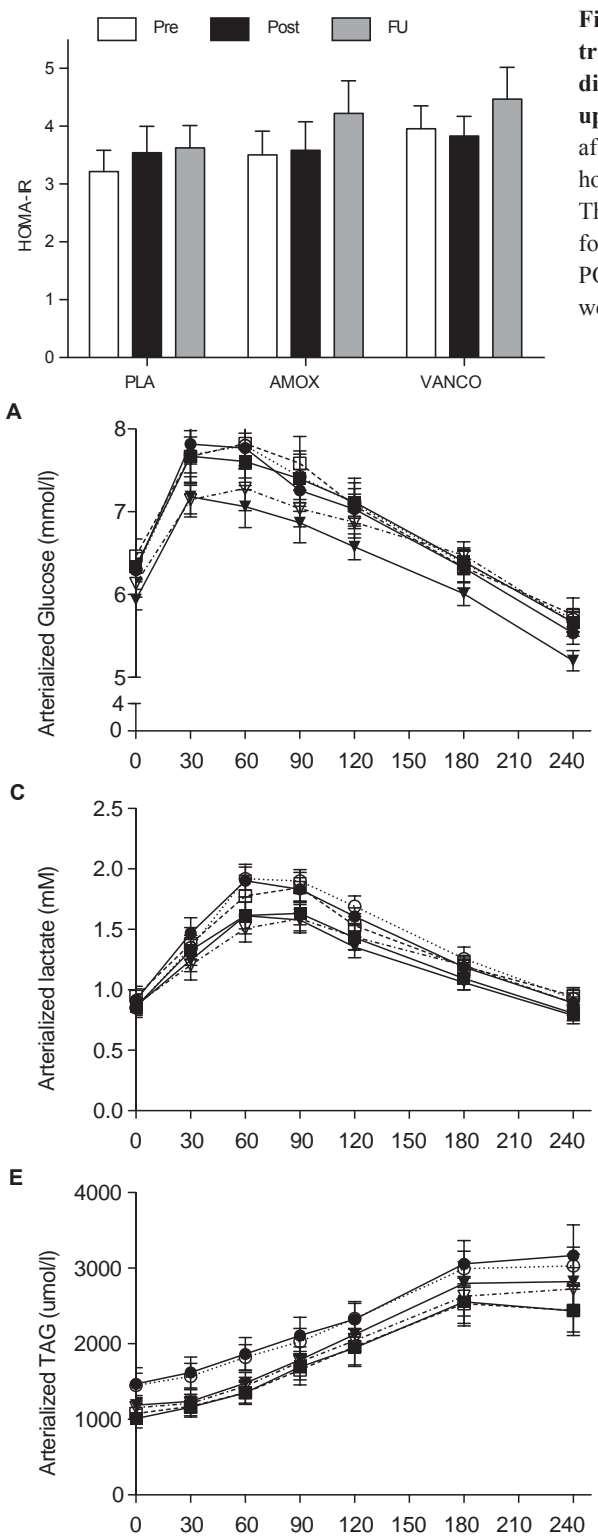

Figure S2.2. The effect of vancomycin, amoxicillin and placebo treatment for 7 days on whole-body insulin sensitivity directly after cessation of treatment and at 8-weeks followup. Data are mean \pm SEM $(n=37)$. AMOX and VANCO did not affect whole-body insulin sensitivity, reflected by the homeostasis model assessment of insulin resistance (HOMA-IR). There was a trend for a time-effect, but no timextreatment effect for FU compared to baseline ( $\mathrm{P}=0.078)$. PRE, baseline values; POST, values directly after treatment cessation; FU, values at 8weeks follow-up.

\section{$B$}

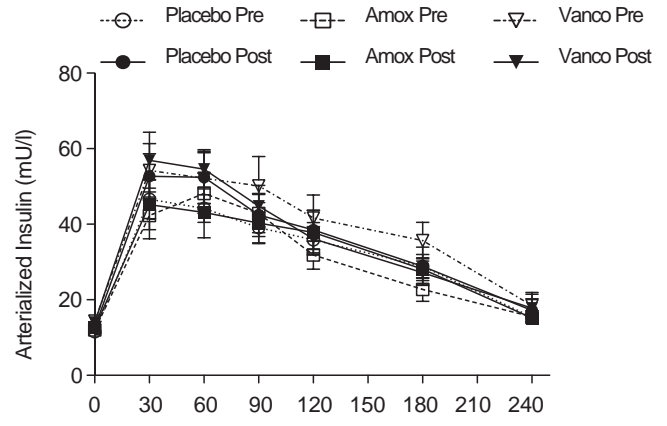

D
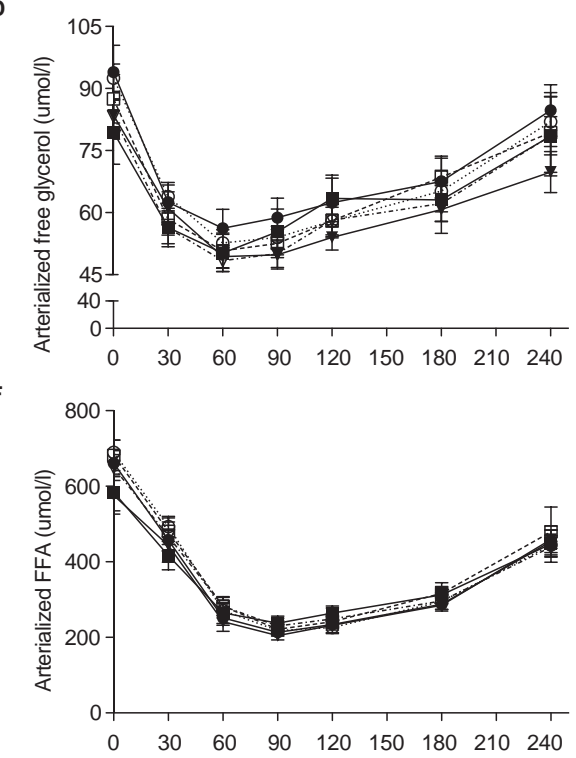

Figure S2.3. The effect of vancomycin, amoxicillin and placebo treatment for 7 days on plasma metabolite concentrations. Data are mean \pm SEM $(n=37)$. Fasting $(t=0)$ and postprandial concentrations of $(A)$ arterialized glucose, (B) lactate, $(C)$ free glycerol, (D) triacylglycerol (TAG) and (E) free fatty acid (FFA) concentrations were not affected by AMOX or VANCO as assessed by repeated measures ANOVA. 


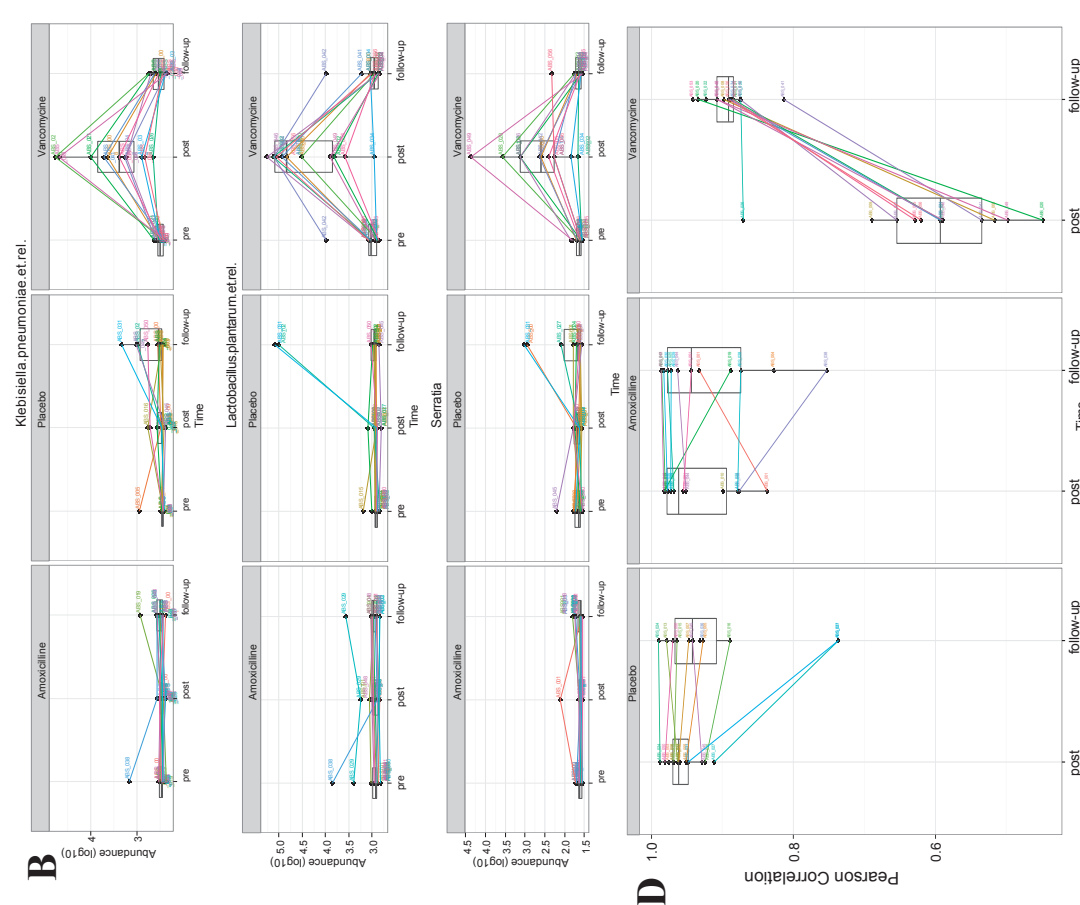

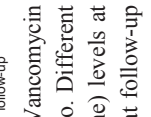

$>$ 웡

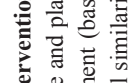

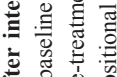

츨

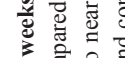

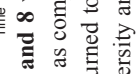

氖的

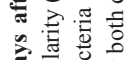

焉票总

今े

잉

请

商 仓

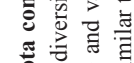

额

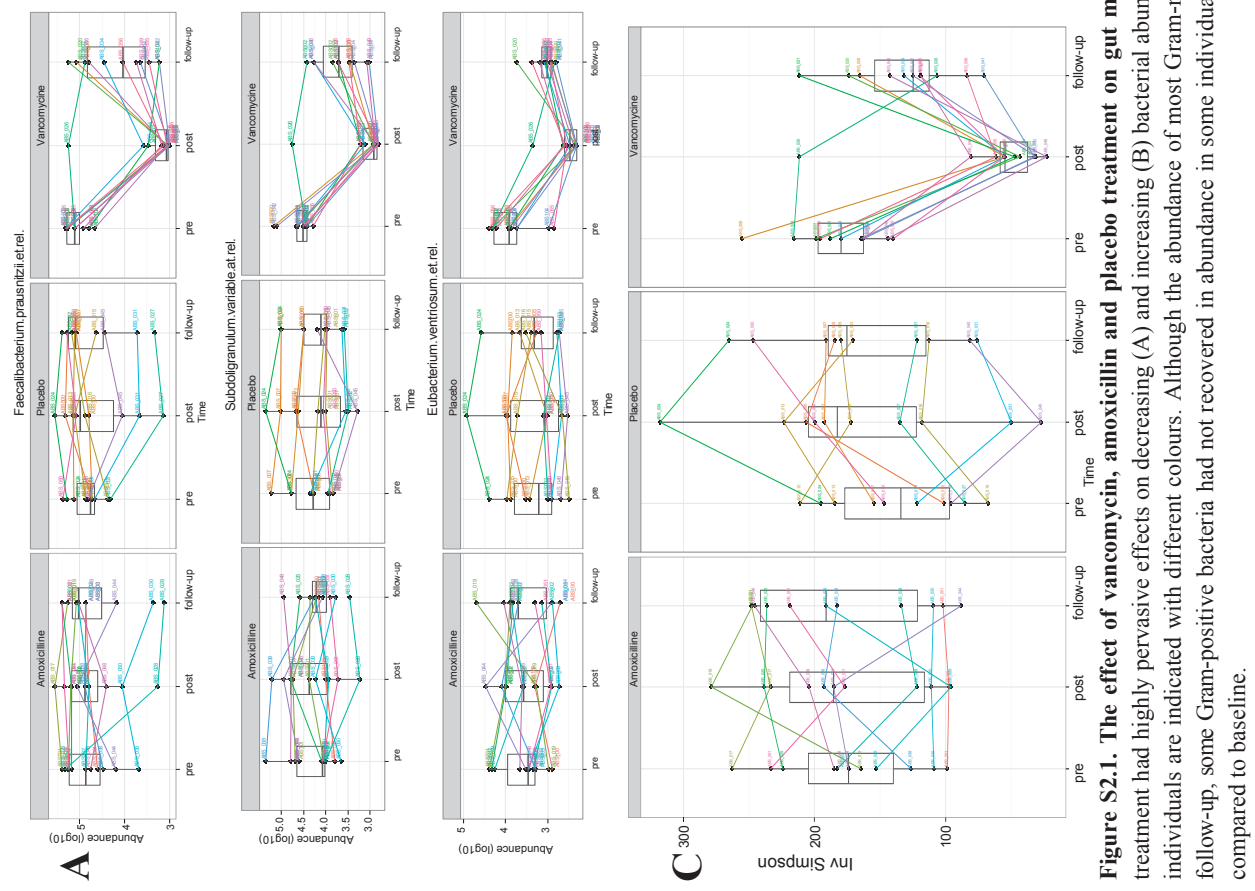



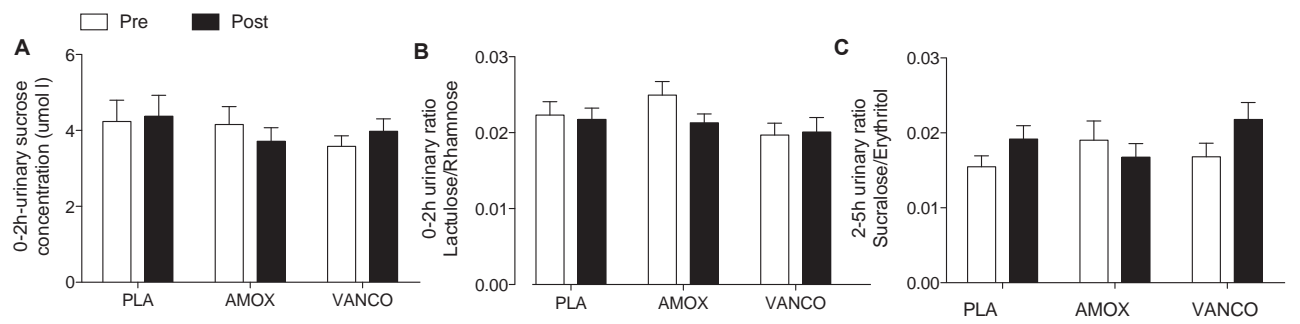

Figure S2.4. The effect of vancomycin, amoxicillin and placebo treatment for 7 days on gut permeability. Bars represent means \pm SEM $(n=56)$. To determine gut permability, urine was collected immediately before drinking a multisaccharide-mix $(\mathrm{t} 0)$, at $\mathrm{t}=120$ and $\mathrm{t}=300$ minutes. AMOX and VANCO did not affect $(\mathrm{A})$ gastroduodenal permeability as expressed by the urinary sucrose concentration at $\mathrm{t}=120 \mathrm{~min}$, (B) small interstine permeability expressed by the lactulose/rhamnose ratio (urine collection 0$120 \mathrm{~min}$ ), (C) proximal colon permeability: suclarose/erythritol ratio (urine collection 120-300 min).

A

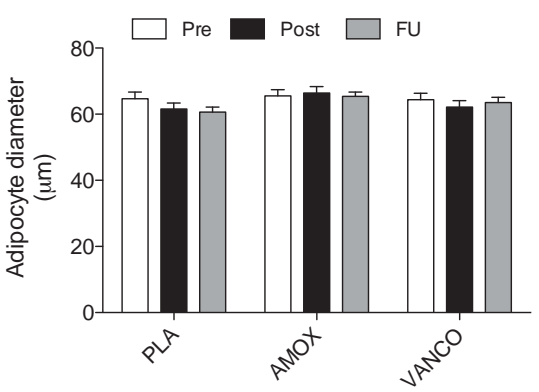

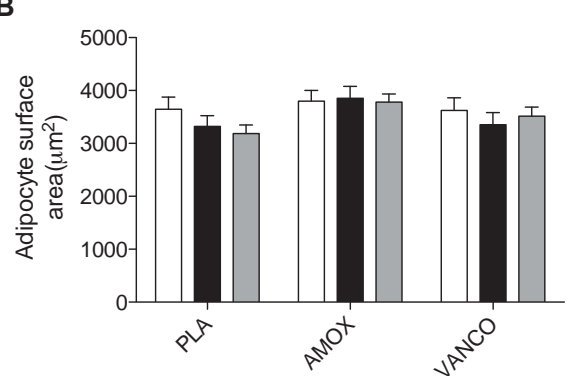

C

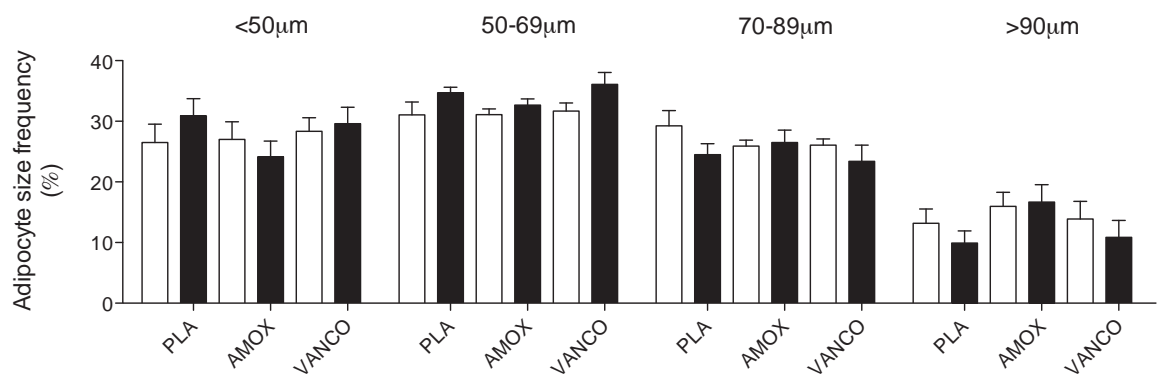

Figure S2.5. Abdominal subcutaneous adipocyte morphology before and immediately after placebo, amoxicillin and vancomycin treatment, as well as after 8 weeks follow-up. Representative sections of stained adipose tissue were used for adipocyte size determination in a subgroup of subjects $(n=18)$. AMOX and VANCO did not affect (A) mean adipocyte diameter, (B) mean adipocyte surface area and (C) adipocyte size distribution. 

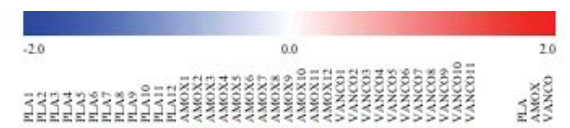

CITRATE.CYCLE.TCA.CYCLE

A

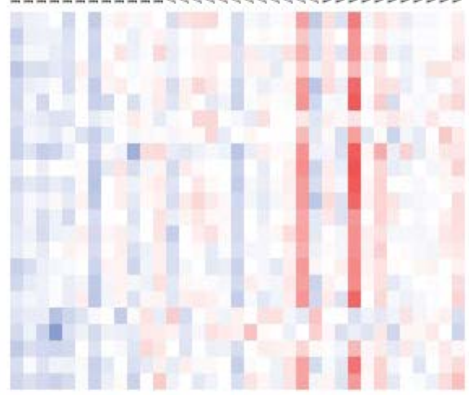

B

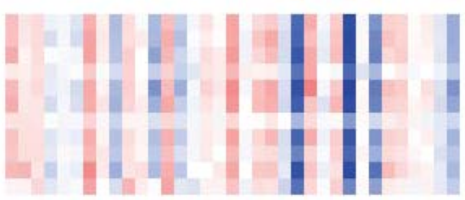

C Upregulated pathways after vancomycin treatment PPAR signaling - FA degradation AA degradation

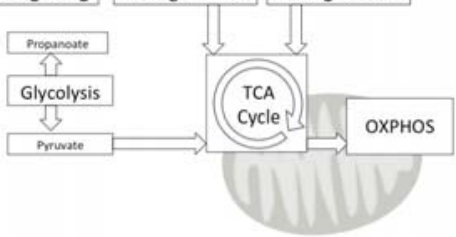

GLYCOLYSIS GLUCONEOGENESI
OXIDATIVE.PHOSPHORYLATION

GALACTOSEMETABOLISM

PYRUVATE.METABOLISM

RETINOL.METABOLIS
STARCH.SUCROSE

ARACHIDONIC.ACID.METABOLISM

ETHER.LIPID METABOLISM

PPAR

PROPANOATE.METABOLISM

CYSTEINE.AND.METHIONINE.METABOLISM

TYCINE. SERINE.AND.THREONINE. METABOLISM

PROTEIN.DIGESTION.AND.ABSORPTION

VYROSINE.METABOLISM

ANTIGEN.PROCESSING.AND.PRESENTATION
INTESTINAL.IMMUNE.NETWORK.FOR.IGA.PRODUCTION

LYSOSOME

DRUG.METABOLISM.CYTOCHROME.P450

MINERAL.ABSORPTION

NATURAL.KILLER.CELL.MEDIATED.CYTOTOXICTTY

B.CELL.RECEPTOR.SIGNALING.PATHWAY
TCELL.RECEPTOR.SIGNALING.PATHWAY

T.CELL RECEPTOR.SIGNALI
ALLOGRAFT REEECTION

PRIMARY.IMMUNODDEFICIENCY

HEMATOPOETIC.CELL.LINEAGE

APOPTOS

TNF

NOD

Downregulated pathways after vancomycin treatment

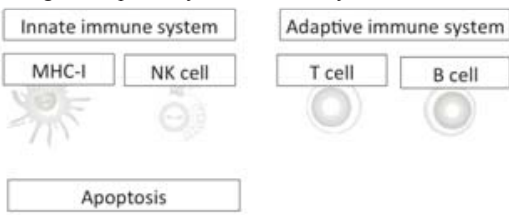

Figure S2.6. Heatmap of 7 days placebo, amoxicillin and vancomycin-induced effects on abdominal subcutaneous adipose tissue gene expression. (A) Upregulated genes, (B) downregulated genes determined in adipose tissue biopsies $(n=30)$. The heatmap shows pathways related to metabolic function (Kyoto Encyclopedia of Genes and Genomes (KEGG) database) derived from Gene Set Enrichment Analysis. Signal log-ratio's (SLR) of genes in the heatmap represent genes that significantly contribute to the described pathways (FDR q-value $<0.2$ ). Color in the heatmap reflects the SLR per subject and group, with blue color being downregulated and red color being upregulated genes following treatment. (C) Schematic illustration of pathways that are upregulated and downregulated after vancomycin treatment as compared to placebo. 
Table S2.1 (related to Figure 1). Significantly different microbial taxa after 7 days intervention with vancomycin and placebo in feces using linear mixed models

\begin{tabular}{|c|c|c|c|c|}
\hline & $\begin{array}{r}\mathrm{BH}_{-} \\
\text {adjusted }\end{array}$ & $P$ value & $\begin{array}{l}\text { log fold change } \\
\text { within vancomycine }\end{array}$ & $\begin{array}{l}\text { log fold change } \\
\text { within placebo }\end{array}$ \\
\hline Coprococcus eutactus et rel. & $1.46 \mathrm{E}-02$ & $1.91 \mathrm{E}-03$ & 1.62262964 & -0.036098988 \\
\hline Ruminococcus bromii et rel. & $4.57 \mathrm{E}-02$ & $1.09 \mathrm{E}-02$ & 1.52679587 & -0.338380905 \\
\hline Faecalibacterium prausnitzii et rel. & $1.52 \mathrm{E}-02$ & $2.35 \mathrm{E}-03$ & 1.4508797 & 0.075849958 \\
\hline Lactobacillus plantarum et rel. & $6.98 \mathrm{E}-05$ & $5.37 \mathrm{E}-07$ & -1.39338187 & -0.026993041 \\
\hline Eubacterium hallii et rel. & $2.96 \mathrm{E}-03$ & $1.25 \mathrm{E}-04$ & 1.33243286 & -0.114103865 \\
\hline Ruminococcus obeum et rel. & $1.20 \mathrm{E}-02$ & $1.29 \mathrm{E}-03$ & 1.25172537 & -0.051553 \\
\hline Lachnospira pectinoschiza et rel. & $1.07 \mathrm{E}-02$ & $1.07 \mathrm{E}-03$ & 1.19017372 & -0.144882415 \\
\hline Clostridium cellulosi et rel. & $9.21 \mathrm{E}-03$ & $8.50 \mathrm{E}-04$ & 1.14016546 & -0.267960043 \\
\hline Clostridium nexile et rel. & $1.51 \mathrm{E}-02$ & $2.21 \mathrm{E}-03$ & 1.103251 & -0.158269081 \\
\hline Anaerostipes caccae et rel. & $2.73 \mathrm{E}-02$ & $4.83 \mathrm{E}-03$ & 1.08875593 & -0.225845465 \\
\hline Dorea formicigenerans et rel. & $4.03 \mathrm{E}-02$ & $9.31 \mathrm{E}-03$ & 1.05973413 & -0.123118158 \\
\hline Veillonella & $9.45 \mathrm{E}-04$ & 2.91E-05 & -0.99977749 & 0.008042942 \\
\hline Clostridium sphenoides et rel. & $1.44 \mathrm{E}-02$ & 1.77E-03 & 0.91030989 & -0.036075241 \\
\hline Serratia & $3.30 \mathrm{E}-02$ & $7.11 \mathrm{E}-03$ & -0.85859596 & -0.132395735 \\
\hline Papillibacter cinnamivorans et rel. & $3.21 \mathrm{E}-02$ & $6.66 \mathrm{E}-03$ & 0.82835965 & -0.156871014 \\
\hline Klebisiella pneumoniae et rel. & $1.92 \mathrm{E}-02$ & $3.15 \mathrm{E}-03$ & -0.8215981 & -0.144113787 \\
\hline Megasphaera elsdenii et rel. & $7.84 \mathrm{E}-04$ & $1.81 \mathrm{E}-05$ & -0.8001288 & -0.016009582 \\
\hline Escherichia coli et rel. & $6.70 \mathrm{E}-03$ & $4.64 \mathrm{E}-04$ & -0.79077723 & -0.086406762 \\
\hline Lachnobacillus bovis et rel. & $3.21 \mathrm{E}-02$ & $6.43 \mathrm{E}-03$ & 0.78609501 & -0.130157333 \\
\hline Enterobacter aerogenes et rel. & $3.21 \mathrm{E}-02$ & $6.48 \mathrm{E}-03$ & -0.76953625 & -0.145197198 \\
\hline Anaerovorax odorimutans et rel. & $1.47 \mathrm{E}-02$ & $2.04 \mathrm{E}-03$ & 0.66699492 & -0.054689642 \\
\hline Sutterella wadsworthia et rel. & $3.21 \mathrm{E}-02$ & $6.35 \mathrm{E}-03$ & -0.54038979 & -0.096457386 \\
\hline Granulicatella & $2.96 \mathrm{E}-03$ & $1.37 \mathrm{E}-04$ & -0.49661041 & -0.135840177 \\
\hline Lactobacillus salivarius et rel. & $4.99 \mathrm{E}-02$ & $1.23 \mathrm{E}-02$ & -0.3206781 & 0.003000548 \\
\hline Collinsella & $7.84 \mathrm{E}-04$ & $1.72 \mathrm{E}-05$ & 0.30211314 & -0.045063251 \\
\hline Eubacterium cylindroides et rel. & $3.56 \mathrm{E}-02$ & 7.93E-03 & -0.28758184 & -0.020381399 \\
\hline Clostridium difficile et rel. & $5.00 \mathrm{E}-03$ & 3.07E-04 & -0.26460033 & -0.082852343 \\
\hline Bifidobacterium & $1.27 \mathrm{E}-02$ & $1.47 \mathrm{E}-03$ & 0.18320543 & -0.092371693 \\
\hline Eggerthella lenta et rel. & $7.26 \mathrm{E}-03$ & $6.14 \mathrm{E}-04$ & 0.15800988 & -0.079697689 \\
\hline Aerococcus & $1.92 \mathrm{E}-02$ & $3.25 \mathrm{E}-03$ & -0.06422419 & -0.034350517 \\
\hline Atopobium & $3.79 \mathrm{E}-03$ & $2.04 \mathrm{E}-04$ & 0.02943863 & 0.005546336 \\
\hline
\end{tabular}

This table shows bacteria taxa that were significantly different after placebo or vancomycin treatment using linear mixed models in addition to between-group analysis as determined by paired Wilxocon test. BH-adjustment: Benjamini-Hochberg corrected 
Table S2.2. The effect of 7 days vancomycin treatment confirmed by Random Forests analysis.

\begin{tabular}{|c|c|c|c|}
\hline Bacteria & MDA & Bacteria & MDA \\
\hline Klebsiella pneumoniae et rel. & $2,15 E-02$ & Bacteroides stercoris et rel. & $1,94 E-03$ \\
\hline Clostridium leptum et rel. & $1,33 E-02$ & Outgrouping Clostridium cluster XIVa & $1,89 E-03$ \\
\hline Clostridium nexile et rel. & $1,05 E-02$ & Streptococcus mitis et rel. & $1,68 E-03$ \\
\hline Lachnobacillus bovis et rel. & $9,86 E-03$ & Yersinia et rel. & $1,62 E-03$ \\
\hline Megasphaera elsdenii et rel. & $9,51 E-03$ & Bryantella formatexigens et rel. & $1,58 E-03$ \\
\hline Serratia & $9,40 E-03$ & Subdoligranulum variable at rel. & $1,49 E-03$ \\
\hline Veillonella & $9,20 E-03$ & Clostridium sphenoides et rel. & $1,48 E-03$ \\
\hline Eubacterium hallii et rel. & $8,77 E-03$ & Papillibacter cinnamivorans et rel. & $1,33 E-03$ \\
\hline Clostridium symbiosum et rel. & $8,58 E-03$ & Granulicatella & $1,21 E-03$ \\
\hline Prevotella tannerae et rel. & $8,40 E-03$ & Streptococcus intermedius et rel. & $1,15 E-03$ \\
\hline Uncultured Clostridiales I & $8,34 E-03$ & Eubacterium biforme et rel. & $1,13 E-03$ \\
\hline Lachnospira pectinoschiza et rel. & $7,44 E-03$ & Clostridium stercorarium et rel. & $1,11 E-03$ \\
\hline Ruminococcus gnavus et rel. & $7,22 E-03$ & Coprococcus eutactus et rel. & $8,78 E-04$ \\
\hline Ruminococcus lactaris et rel. & $6,77 E-03$ & Faecalibacterium prausnitzii et rel. & $6,84 E-04$ \\
\hline Enterobacter aerogenes et rel. & $5,39 E-03$ & Eubacterium rectale et rel. & $6,58 E-04$ \\
\hline Dorea formicigenerans et rel. & $5,34 E-03$ & Actinomycetaceae & $6,53 E-04$ \\
\hline Uncultured Clostridiales II & $4,61 E-03$ & Streptococcus bovis et rel. & $6,21 E-04$ \\
\hline Sporobacter termitidis et rel. & $4,45 E-03$ & Coprobacillus catenaformis et rel. & $6,10 E-04$ \\
\hline Lactobacillus plantarum et rel. & $4,37 E-03$ & Bacteroides splachnicus et rel. & $5,73 E-04$ \\
\hline Bacteroides vulgatus et rel. & $3,98 E-03$ & Uncultured Mollicutes & $5,58 E-04$ \\
\hline Eubacterium ventriosum et rel. & $3,74 E-03$ & Anaerofustis & $5,53 E-04$ \\
\hline Bacteroides plebeius et rel. & $3,64 E-03$ & Eubacterium cylindroides et rel. & $5,34 E-04$ \\
\hline Butyrivibrio crossotus et rel. & $3,46 E-03$ & Aneurinibacillus & $5,03 E-04$ \\
\hline Anaerostipes caccae et rel. & $3,41 E-03$ & Bacteroides ovatus et rel. & $4,95 E-04$ \\
\hline Anaerovorax odorimutans et rel. & $3,08 E-03$ & Methylobacterium & $3,98 E-04$ \\
\hline Clostridium orbiscindens et rel. & $3,02 E-03$ & Eubacterium siraeum et rel. & $3,58 E-04$ \\
\hline Escherichia coli et rel. & $3,01 E-03$ & Peptococcus niger et rel. & $2,72 E-04$ \\
\hline Anaerotruncus colihominis et rel. & $2,98 E-03$ & Collinsella & $2,48 E-04$ \\
\hline Clostridium cellulosi et rel. & $2,87 E-03$ & Prevotella melaninogenica et rel. & $2,38 E-04$ \\
\hline Bacteroides uniformis et rel. & $2,71 E-03$ & Eggerthella lenta et rel. & $2,37 E-04$ \\
\hline Ruminococcus obeum et rel. & $2,68 E-03$ & Catenibacterium mitsuokai et rel. & $2,27 E-04$ \\
\hline Ruminococcus bromii et rel. & $2,47 E-03$ & Burkholderia & $1,87 E-04$ \\
\hline Oscillospira guillermondii et rel. & $2,40 E-03$ & Vibrio & $1,74 E-04$ \\
\hline Roseburia intestinalis et rel. & $2,39 E-03$ & Uncultured Chroococcales & $1,38 E-04$ \\
\hline Bacteroides intestinalis et rel. & $2,12 E-03$ & Brachyspira & $1,35 E-04$ \\
\hline Ruminococcus callidus et rel. & $1,99 E-03$ & & \\
\hline
\end{tabular}

This table shows the mean decrease accuracy of bacterial taxa that were also found to be altered after 7 days vancomycin treatment as determined by paired Wilcoxon test. MDA (mean decrease accuracy) represents the impact of bacterial taxa on the accuracy of the model. 
Table S2.3. Effects of vancomycin and amoxicillin vs. placebo on gene set enrichment of the adipose tissue

\begin{tabular}{|c|c|c|c|c|c|}
\hline \multirow{2}{*}{$\begin{array}{l}\text { CLASS } \\
\text { KEGG BRITE }\end{array}$} & \multirow{2}{*}{$\begin{array}{l}\text { UPREGULATED GENE SETS } \\
\text { KEGG PATHWAYS }\end{array}$} & \multicolumn{2}{|c|}{ VANCO-PLA } & \multicolumn{2}{|c|}{ AMOX-PLA } \\
\hline & & NES & FDR-q & NES & FDR-q \\
\hline $\begin{array}{l}\text { Energy } \\
\text { metabolism }\end{array}$ & OXIDATIVE.PHOSPHORYLATION & $2.24 *$ & 0.000 & $1.75^{*}$ & 0.081 \\
\hline \multirow{6}{*}{$\begin{array}{l}\text { Carbohydrate } \\
\text { metabolism }\end{array}$} & GLYCOLYSIS.GLUCONEOGENESIS & $1.95^{*}$ & 0.006 & $1.81^{*}$ & 0.062 \\
\hline & GALACTOSE.METABOLISM & $1.76^{*}$ & 0.023 & $2.02 *$ & 0.029 \\
\hline & PYRUVATE.METABOLISM & $1.99 *$ & 0.006 & 1.38 & 0.263 \\
\hline & CITRATE.CYCLE.TCA.CYCLE. & $1.83 *$ & 0.016 & $1.86^{*}$ & 0.049 \\
\hline & PROPANOATE.METABOLISM & $1.89 *$ & 0.010 & 1.39 & 0.258 \\
\hline & STARCH.AND.SUCROSE.METABOLISM & 1.51 & 0.113 & $1.74^{*}$ & 0.079 \\
\hline \multirow{4}{*}{$\begin{array}{l}\text { Lipid } \\
\text { metabolism }\end{array}$} & ARACHIDONIC.ACID.METABOLISM & 0.97 & 0.719 & $1.74^{*}$ & 0.081 \\
\hline & FATTY.ACID.DEGRADATION & $1.86^{*}$ & 0.014 & 1.14 & 0.522 \\
\hline & ETHER.LIPID.METABOLISM & $1.84 *$ & 0.015 & 0.94 & 0.804 \\
\hline & PPAR.SIGNALING.PATHWAY & $1.89 *$ & 0.010 & 0.85 & 0.889 \\
\hline \multirow{7}{*}{$\begin{array}{l}\text { Amino acid } \\
\text { metabolism }\end{array}$} & LYSINE.DEGRADATION & $1.87^{*}$ & 0.012 & 0.89 & 0.852 \\
\hline & CYSTEINE.AND.METHIONINE.METABOLISM & $1.89 *$ & 0.010 & $1.76^{*}$ & 0.078 \\
\hline & TYROSINE.METABOLISM & $1.83 *$ & 0.016 & $1.72 *$ & 0.083 \\
\hline & $\begin{array}{l}\text { VALINE.LEUCINE.AND.ISOLEUCINE.DEGRADATI } \\
\text { ON }\end{array}$ & $1.79 *$ & 0.018 & 1.59 & 0.144 \\
\hline & $\begin{array}{l}\text { GLYCINE.SERINE.AND.THREONINE.METABOLIS } \\
\text { M }\end{array}$ & $1.73^{*}$ & 0.030 & 1.6 & 0.137 \\
\hline & LYSOSOME & $1.99 *$ & 0.006 & $2.22 *$ & 0.000 \\
\hline & COMPLEMENT.AND.COAGULATION.CASCADES & $2.21 *$ & 0.001 & 1.6 & 0.133 \\
\hline \multirow{2}{*}{$\begin{array}{l}\text { Digestive } \\
\text { system }\end{array}$} & PROTEIN.DIGESTION.AND.ABSORPTION & $2.01 *$ & 0.006 & n.a. & n.a. \\
\hline & MINERAL.ABSORPTION & $1.86^{*}$ & 0.013 & $1.79 *$ & 0.068 \\
\hline Immune system & $\begin{array}{l}\text { INTESTINAL.IMMUNE.NETWORK.FOR.IGA.PROD } \\
\text { UCTION }\end{array}$ & n.a. & n.a. & $2 *$ & 0.027 \\
\hline (MHC-II) & ANTIGEN.PROCESSING.AND.PRESENTATION & n.a. & n.a. & $1.8^{*}$ & 0.059 \\
\hline \multirow{3}{*}{$\begin{array}{l}\text { Xenobiotics } \\
\text { metabolism }\end{array}$} & DRUG.METABOLISM.CYTOCHROME.P450 & $2.09 *$ & 0.003 & 1.61 & 0.131 \\
\hline & $\begin{array}{l}\text { METABOLISM.OF.XENOBIOTICS.BY.CYTOCHRO } \\
\text { ME.P450 }\end{array}$ & $1.98 *$ & 0.006 & 1.42 & 0.237 \\
\hline & DRUG.METABOLISM.OTHER.ENZYMES & 1.07 & 0.621 & $1.68^{*}$ & 0.100 \\
\hline $\begin{array}{l}\text { Cofactor and } \\
\text { vitamin }\end{array}$ & RETINOL.METABOLISM & $1.99 *$ & 0.006 & 1.12 & 0.560 \\
\hline $\begin{array}{l}\text { Signaling } \\
\text { molecules }\end{array}$ & ECM.RECEPTOR.INTERACTION & $1.77 *$ & 0.021 & 0.82 & 0.920 \\
\hline
\end{tabular}




\begin{tabular}{|c|c|c|c|c|c|}
\hline \multirow{2}{*}{$\begin{array}{l}\text { CLASS } \\
\text { KEGG BRITE }\end{array}$} & \multirow{2}{*}{$\begin{array}{l}\text { DOWNREGULATED GENE SETS } \\
\text { KEGG PATHWAYS }\end{array}$} & \multicolumn{2}{|c|}{ VANCO-PLA } & \multicolumn{2}{|c|}{ AMOX-PLA } \\
\hline & & NES & FDR- & NES & FDR-q \\
\hline \multirow[t]{5}{*}{ Immune system } & $\begin{array}{l}\text { NATURAL.KILLER.CELL.MEDIATED.CYTOTOXIC } \\
\text { ITY }\end{array}$ & $-2.4^{*}$ & 0.001 & -0.74 & 1.000 \\
\hline & B.CELL.RECEPTOR.SIGNALING.PATHWAY & $-1.8^{*}$ & 0.026 & -0.76 & 1.000 \\
\hline & T.CELL.RECEPTOR.SIGNALING.PATHWAY & $-2.23 *$ & 0.002 & -0.77 & 1.000 \\
\hline & NOD.LIKE.RECEPTOR.SIGNALING.PATHWAY & $-2.24 *$ & 0.003 & -1 & 0.831 \\
\hline & HEMATOPOIETIC.CELL.LINEAGE & $-1.72 *$ & 0.039 & n.a. & n.a. \\
\hline Immune diseases & ALLOGRAFT.REJECTION & $-1.55^{*}$ & 0.078 & n.a. & n.a. \\
\hline \multirow[t]{4}{*}{ (MHC-I) } & GRAFT.VERSUS.HOST.DISEASE & $-1.66^{*}$ & 0.053 & n.a. & n.a. \\
\hline & PRIMARY.IMMUNODEFICIENCY & $-2.08 *$ & 0.006 & n.a. & n.a. \\
\hline & INFLAMMATORY.BOWEL.DISEASE.IBD. & $-1.55^{*}$ & 0.079 & n.a. & n.a. \\
\hline & $\begin{array}{l}\text { SYST.L.ERYTHEM. (GENES } \\
\text { CLUSTER) }\end{array}$ & $-1.71 *$ & 0.040 & -1.35 & 0.489 \\
\hline $\begin{array}{l}\text { Substance } \\
\text { dependence }\end{array}$ & ALCOHOLISM (GENES OF HISTONE CLUSTER) & $-1.85^{*}$ & 0.023 & $-1.88^{*}$ & 0.085 \\
\hline $\begin{array}{l}\text { Replication and } \\
\text { repair }\end{array}$ & DNA.REPLICATION & $-1.57 *$ & 0.072 & n.a. & n.a. \\
\hline $\begin{array}{l}\text { Cell growth and } \\
\text { death }\end{array}$ & APOPTOSIS & $-1.99 *$ & 0.010 & n.a. & n.a. \\
\hline \multirow{2}{*}{$\begin{array}{l}\text { Signal } \\
\text { transduction }\end{array}$} & NF.KAPPA.B.SIGNALING.PATHWAY & $-1.56^{*}$ & 0.076 & n.a. & n.a. \\
\hline & TNF.SIGNALING.PATHWAY & $-1.63 *$ & 0.061 & n.a. & n.a. \\
\hline
\end{tabular}

Gene set enrichment analysis found enriched pathways of the KEGG (Kyoto Encyclopedia of Genes and Genomes) database. KEGG BRITE denotes clustering of KEGG-pathways in functional hierarchies, NES normalized enrichment score, FDR-q false discovery rate adjusted p-value, not appliccable means no significant upregulation, respectively downregulation after vancomycin or amoxicillin of the selected pathway. Data of $n=10$ per group was used for microarray analysis. * FDR-q $<0.1$, significant enrichment of the selected pathway. 


\section{Chapter 5}

Limited, individual- and cohort-specific patterns of gut microbiota composition associated with tissue-specific insulin sensitivity in overweight and obese males

Gerben D.A. Hermes*, Dorien Reijnders*, Ruud S. Kootte, Gijs H. Goossens, Hauke Smidt, Max Nieuwdorp, Ellen E. Blaak, Erwin G. Zoetendal

*These authors contributed equally to this work 


\begin{abstract}
Background: A growing body of evidence suggests that the human gut microbiota plays a role in the development of obesity and related metabolic diseases. However, there is little consensus between studies, which could be due to biological as well as technical variation. In addition, no human data are available to investigate whether tissue-specific insulin sensitivity is related to a specific microbial pattern. Aim: To examine the relation between microbiota composition at the genus level, and tissue-specific insulin sensitivity in two independent cohorts of overweight and obese pre-diabetic men. Methods: Fecal microbiota composition was characterized using the HITChip, a 16S ribosomal RNA gene targeted microarray. In all subjects, hepatic, peripheral and adipose tissue insulin sensitivity were determined by a two-step hyperinsulinemic-euglycemic clamp with $\left[6,6-{ }^{2} \mathrm{H}_{2}\right]$-glucose tracer infusion. Random forest classification and linear and logistic regression analysis were used to investigate the relation between bacterial abundance at the genus level, and tissuespecific insulin sensitivity. Results: Despite the strongly subject-specific microbiota composition, we found some associations of microbial groups with host parameters. However, these associations were cohort-specific due to pronounced variation in microbiota composition between cohorts, suggesting the existence of alternative states for dysbiosis in metabolic syndrome patients. Remarkably, in neither of the cohorts there was any relationship between microbial composition and tissue-specific insulin sensitivity. Conclusion: Our findings do not predict a physiologically significant role of the gut microbiota in host insulin sensitivity when the overweight and/or obese state has already developed and argue that care should be taken when significant correlations from single cohorts are extrapolated into generalized biological relevance.
\end{abstract}




\section{Introduction}

There is increasing evidence to suggest that our gut microbiome is associated with the development of obesity, insulin resistance and diabetes mellitus type 2 (T2DM). This concept was first described in studies, in which germ-free mice showed less adiposity, improved insulin sensitivity and glucose tolerance as compared to conventionalized mice $e^{103,}$ 311-313. Later studies showed that the microbiota composition of lean, obese, diabetic vs. healthy mice and humans differ ${ }^{48,62,182,314}$. Microbial analyses of large genome-wide association studies showed that patients with T2DM are characterized by a decrease in the abundance of universal butyrate-producers and an increase in various opportunistic pathogens ${ }^{182,195,314}$. Nevertheless, between studies there is an overall lack of consistency regarding the identified microbial biomarkers and putative mechanisms underlying the observations. Discrepancies between studies could be a result of the heterogeneity of groups, genetic background, habitual lifestyle, diet and the methodology used by different researchers ${ }^{232}$. Meta-analyses have shown that individuals could be classified based on their microbiota as lean or obese with statistically significant accuracy within a study, consistent with the observation that this phenotype can be experimentally transferred in mice by microbiota transplantation ${ }^{48}$. However, microbial signatures were not consistent between studies even when the data were analysed in the same way ${ }^{47}$. Furthermore, the comparison of discriminant metagenomic markers for T2DM in European women ${ }^{314}$ and Chinese individuals $^{195}$ revealed cohort specific differences, and the authors concluded that metagenomic predictive tools for T2DM should be specific for the age and geographical location of the populations studied ${ }^{314}$. Correspondingly, another putative marker for obesity based on the gut microbiome, the Bacteroidetes to Firmicutes ratio (B:F) proposed by Ley et $a l^{62}$, remains controversial as it has been confirmed ${ }^{36}$, refuted ${ }^{170}$ and even contradicted $^{173}$.

Nevertheless, transplantation of the microbiota in rodents and humans has provided evidence for the causal role of the microbiota in adiposity and metabolic health. Fecal transplantation from obese into germ-free mice significantly increased adiposity ${ }^{48,315}$, and fecal transplantation from lean donors into metabolic syndrome patients altered the recipients' microbiota composition with a concomitant, minor improvement in peripheral insulin sensitivity based on responders and non-responders ${ }^{90}$. In contrast, we have recently demonstrated that interference with adult microbiota by 7-day antibiotic treatment has no clinically relevant impact on host metabolism in obese humans, despite deviant microbiota $^{69}$. Importantly, the relationship between gut microbiota and tissue-specific insulin action has never been established in obese humans. Here, we investigated the relationship between the gut microbiota composition and adipose tissue, muscle and liver insulin sensitivity by means of the gold-standard two-step hyperinsulinemic-euglycemic clamp with $\left[6,6-{ }^{2} \mathrm{H}_{2}\right]$-glucose tracer infusion in two independent cohorts of men suffering from metabolic syndrome. 


\section{Methods}

\section{Study population}

We investigated baseline microbiota composition in relation to tissue-specific insulin sensitivity and other indicators of glucose metabolism in two independent cohorts of overweight and obese (BMI 25-45 kg/m²) Caucasian men between 35-70 years old (ClinicalTrials.gov NCT02241421 and Dutch Trial Register NTR2705). The cohort from Maastricht (MAA) consisted of 56 subjects with impaired fasting glucose levels (glucose concentration $\geq 5.6 \mathrm{mmol} / \mathrm{l}$ ) and/or impaired glucose tolerance (IGT, 2h plasma glucose during a $75 \mathrm{~g}$ oral glucose tolerance test 7.8-11.1 mmol/1. The cohort from Amsterdam (AMS) consisted of 42 subjects diagnosed with the metabolic syndrome (according to the NCEP criteria) $)^{316}$. Subjects were not allowed to have used antibiotics three months prior to participation. All subjects gave written informed consent before participation after reading the study protocol, which was reviewed and approved by the Local Medical Ethics Committees. All procedures were performed according to the declaration of Helsinki (revised version, 2008, Seoul, South Korea).

\section{Study Design}

Study measurements were conducted following a 10h overnight fast. The primary outcome of this study was tissue-specific insulin sensitivity (insulin-mediated glucose disposal $(\mathrm{Rd})$ ), hepatic insulin sensitivity (insulin-mediated suppression of endogenous glucose production (\% suppression EGP)), adipose tissue (AT) insulin sensitivity (insulin-mediated suppression of plasma free fatty acids (\% suppression FFA)) as determined by a two-step hyperinsulinemic-euglycemic clamp with $\left[6,6-{ }^{2} \mathrm{H}_{2}\right]$-glucose infusion. To this end, one cannula was inserted into the antecubital vein, whereas a second Teflon cannula was inserted into a superficial dorsal hand vein for blood sampling, which was arterialized by placing the hand into a hot-box, blowing warm air $\left(\sim 50^{\circ} \mathrm{C}\right)$. In the Maastricht cohort, after a bolus-injection of $2.4 \mathrm{mg} \mathrm{kg}^{-1}$ was infused, continuous tracer-infusion was started at 0.04 $\mathrm{mg} \mathrm{kg}^{-1} \mathrm{~min}^{-1}$ and continued throughout the measurement. After $2 \mathrm{~h}$, low-dose insulin was infused at $10 \mathrm{mU} \mathrm{m}^{-2} \mathrm{~min}^{-1}$ for $2 \mathrm{~h}^{272}$, followed by high-dose insulin at $40 \mathrm{mU} \mathrm{m}^{-2} \mathrm{~min}^{-1}$ for $2 \mathrm{~h}$. By variable co-infusion of a $17.5 \%$-glucose solution, enriched by $1.1 \%\left[6,6-{ }^{2} \mathrm{H}_{2}\right]-$ glucose-tracer ${ }^{273}$, plasma concentrations were maintained at $5.0 \mathrm{mmol} / \mathrm{l}$. In the cohort in Amsterdam, insulin was infused at $20 \mathrm{mU} \mathrm{m}^{-2} \mathrm{~min}^{-1}$ for $2 \mathrm{~h}$, followed by $60 \mathrm{mU} \mathrm{m} \mathrm{min}^{-1}$ for $2 \mathrm{~h}^{317}$. For calculation of steady-state-kinetics, the last 30 minutes of each step $(0,10$ and $40 \mathrm{mU} \mathrm{m}^{-2} \mathrm{~min}^{-1}$ insulin) and the last 20 minutes of each step $\left(0,20\right.$ and $60 \mathrm{mU} \mathrm{m}^{-2} \mathrm{~min}$ 1 insulin), respectively, were used for the two cohorts, during which additional blood samples were taken.

In addition, we collected fasting plasma samples to determine insulin and glucose concentrations for the calculation of the homeostasis model assessment for insulin resistance $(\mathrm{HOMA}-\mathrm{IR},[($ fasting insulin $(\mu \mathrm{IU} / \mathrm{ml}) \times$ fasting glucose $(\mathrm{mmol} / \mathrm{l})) / 22.5)]$ and $\beta$ - 
cell function (HOMA-B\%, [20 $\times$ (fasting insulin)/(fasting glucose $\quad-\quad 3.5)]$. Anthropometrical measurements were performed for the calculation of the body mass index (BMI, [weight $(\mathrm{kg}) /$ height $\left.(\mathrm{m})^{2}\right]$ and waist/hip ratio.

\section{Biochemical analysis}

Blood was collected into pre-chilled tubes, centrifuged at $1000 \mathrm{~g}$, and plasma was snapfrozen and stored at $-80^{\circ} \mathrm{C}$ until analyses. Isotopic enrichment of plasma glucose was determined by electron ionization gas chromatography-mass spectrometry and expressed as tracer-to-tracer ratio for steady-state calculations of rate of disappearance (Rd) and endogenous glucose production (EGP). Plasma glucose and glycerol were determined with the Cobas Fara auto-analyzer, Roche, Switzerland). Plasma insulin was measured with a double antibody radioimunoassay (Millipore, MA, USA), and plasma FFA concentrations were analyzed using standard enzymatic techniques automated on a Cobas Fara autoanalyzer (Roche).

\section{Fecal microbiota characterization}

The fecal microbiota composition was determined by analyzing 16S rRNA gene amplicons using the Human Intestinal Tract Chip Microarray (HITChip), a phylogenetic microarray based on 16S rRNA gene sequences of over 1000 intestinal bacterial phylotypes ${ }^{318}$. DNA was isolated from faeces using the repeated bead beating method as previously described, ${ }^{109}$ and subsequently used for microbiota profiling. In short, 16S rRNA genes were amplified by PCR, followed by in vitro transcription, $\mathrm{Cy} 3 / \mathrm{Cy} 5$ labelling and fragmentation of RNA, and hybridization. Duplicate hybridizations with a Pearson correlation $>98 \%$ were considered for further analysis, and microbiota profiles were generated by pre-processing of probe-level measurements with min-max normalization and the frozen-RPA probe summarization $^{319}$ into three phylogenetic levels: order-like, genus-like ( $>90 \%$ sequence similarity), and phylotype-like ( $>98 \%$ sequence similarity $)^{318}$. In the present study the analysis focused on the genus-level variation, referred to as species and relatives ('et rel.'). $\log _{10}$-transformed signals were used as a proxy for bacterial abundance.

\section{Statistical analysis}

To determine the bacterial groups whose relative abundance significantly differed between the two cohorts, a non-paired Wilcoxon test was used. We performed linear regression models (unadjusted and adjusting for age) to determine the association of specific genuslike bacterial groups with the variables under investigation in both cohorts separately. All outcome variables were sorted into quartiles. The highest and lowest range were used for logistic regression and Random Forest classification using the Random Forest and ROCR R packages $^{289,320}$. For all analyses Benjamini-Hochberg $(\mathrm{BH})$ correction was applied for multiple testing. All analyses were performed in R, v3.1.3 $3^{321}$. Principal component analysis was performed in Canoco v $5^{239}$ with $\log 10$ transformed signals summarized to genus like groups. 


\section{Results}

\section{Subject characteristics}

The subjects' characteristics, including insulin resistance measurements are shown in Table 1. Subjects in both the MAA and AMS cohorts were insulin resistant (HOMA-IR: $4.5 \pm 0.2$ and $5.1 \pm 0.3$ respectively, ns). MAA presented with higher fasting glucose concentrations $(6.1 \pm 0.01$ vs. $5.8 \pm 0.09 \mathrm{mmol} / \mathrm{l}, \mathrm{p}<0.05)$, whereas insulin concentrations were lower than in the AMS cohort $(16.8 \pm 0.8$ vs. $20.0 \pm 1.2 \mathrm{mU} / \mathrm{l}, \mathrm{p}<0.05)$. Homeostatic model assessment for ß-cell function (HOMA-B) was lower in MAA than in AMS (136.3 \pm 7.5 vs. $192.0 \pm 15.3$ $\%, \mathrm{p}=0.001)$.

Notably, clamp procedures were slightly different between centers (see Methods), and thus, statistical analysis of potential differences in peripheral, hepatic and adipose tissue insulin sensitivity values between cohorts was not possible.

\section{Fecal microbiota composition}

Remarkably, the average microbiota composition in both cohorts showed pronounced differences (Figure 1). While Actinobacteria (Bifidobacterium and Propionibacterium) were more abundant in the MAA cohort, mainly genus-like groups of Clostridium clusters IV and XIVa were more abundant in the AMS cohort. More specifically, Sporobacter termitidis et rel. (IV), Papillibacter cinnamivorans et rel. (IV), Subdoligranulum variable et rel. (IV), Anaerotruncus colihominis et rel. (IV), Butyrivibrio crossotus et rel. (XIVa) and Clostridium symbiosum et rel. (XIVa), of which the latter four are known to contain butyrate-producing species (Figure 1). In addition to, Uncultered Clostriales I and II.

PCA analysis of the microbiota composition demonstrated that the variation between subjects from MAA $(n=45)$ was higher than those from AMS $(n=40)$ (Figure 2). Although the majority of subjects from MAA overlapped in composition with those of AMS, it is remarkable that approximately $1 / 3$ of MAA showed a distinct composition from this group along the first principal component. This explains the remarkable difference average microbiota composition between the two cohorts. Moreover, it suggests that some metabolic syndrome patients from MAA exhibit an alternative state of microbiota composition compared to the overlapping AMS and MAA individuals. 
Table 1. Subjects' characteristics

\begin{tabular}{llc}
\hline & $\begin{array}{l}\text { MAA (n=56) } \\
\text { Maastricht }\end{array}$ & $\begin{array}{c}\text { AMS (n=42) } \\
\text { Amsterdam }\end{array}$ \\
\hline Age & $59.1 \pm 1.0$ & $54.9 \pm 1.1$ \\
Weight (kg) & $96.5 \pm 1.3$ & $116.3 \pm 2.0$ \\
BMI (kg/m²) & $31.2 \pm 0.4$ & $34.8 \pm 0.5$ \\
Waist/hip ratio & $0.95 \pm 0.01$ & $1.05 \pm 0.01$ \\
Fasting insulin (mU/ml) & $16.80 \pm 0.79$ & $20.02 \pm 1.24$ \\
Fasting glucose (mmol/l) & $6.06 \pm 0.07$ & $5.76 \pm 0.09$ \\
HOMA-IR & $4.5 \pm 0.2$ & $5.1 \pm 0.3$ \\
HOMA-B\% & $136.3 \pm 7.5$ & $192.0 \pm 15.3$ \\
HbA1c (\%) & $5.58 \pm 0.05$ & $5.74 \pm 0.05$ \\
Fasting TAG & $1.30 \pm 0.10$ & $1.50 \pm 0.11$ \\
Rd (umol/kg/min)* & $23.34(10.7-51.4)$ & $26.1(10.1-40.0)$ \\
Suppression EGP (\%)* & $44.1(17.4-79.1)$ & $55.6(30.8-85.0)$ \\
Suppression FFA (\%)* & $45.3(-6,1-84.1)$ & $74.4(53.9-92.1)$ \\
\hline
\end{tabular}

HOMA-IR: homeostasis model assessment for insulin resistance, HOMA- $\beta \%$ : homeostasis model assessment for $\beta$-cell function, HbA1c: glycated haemoglobin, TAG: triacylglycerol, Rd: rate of disappearance, EGP: endogenous glucose production by the liver, FFA: free fatty acids. Data are expressed as mean \pm SEM. Clamp-results are expressed as mean (range).

*due to differences in clamp procedures (see Methods) between centers, no statistical comparison was made between cohorts.

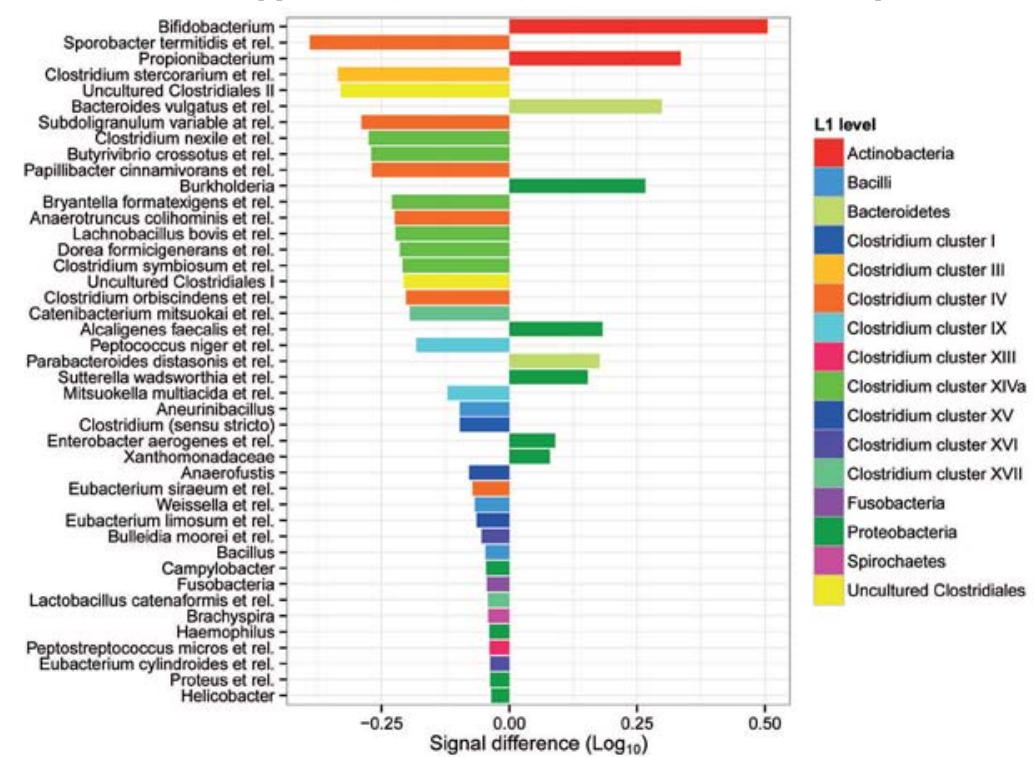

Figure 1. Enrichment of bacterial taxa in two separate cohorts of obese men. Genus like bacterial groups which showed significantly different abundance ( $\log _{10}$ signal intensity) between the two cohorts. The left side shows taxa enriched in AMS right side taxa enriched in MAA. Colors indicate to which Phylum or Class these groups belong. 


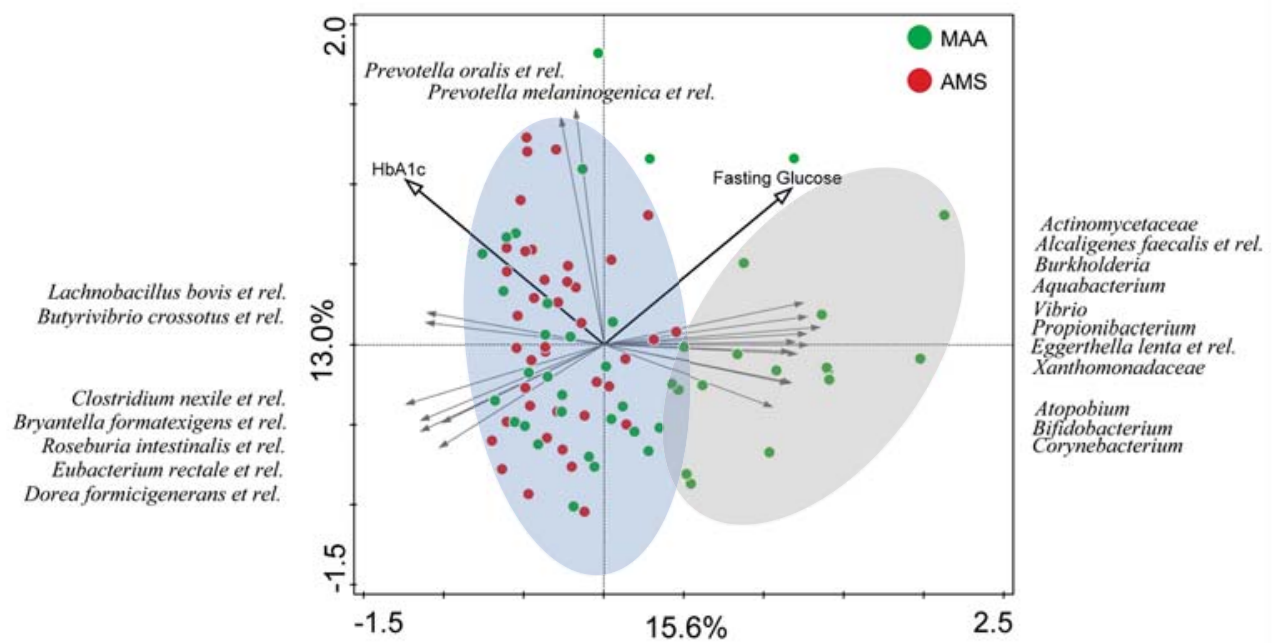

Figure 2. Principle component analysis of the fecal microbiota composition of 85 overweight insulin resistant overweight males from Mastricht (MAA) and Amsterdam (AMS). Individuals from AMS and a subset from MAA overlap and a second group of individuals in MAA was observed as indicated by the light blue shadows. These also show associations with the two metabolic parameters associated with microbiota composition in MAA through Random Forests analysis. The direction of the species arrows depicts the abundance of microbial groups. Length of the arrows is a measure of fit. The environmental variable arrows approximates the correlation between species and an environmental variable. The further a sample falls in the direction indicated by the arrow, the higher the correlation. Samples near the coordinate origin (zero point) suggest near zero correlation.

\section{Correlations between microbiota composition and host metabolic parameters}

\section{Tissue-specific insulin sensitivity}

After correction for multiple testing, peripheral, hepatic and adipose tissue insulin sensitivity ( $\mathrm{Rd}, \%$ suppression of EGP and \% suppression of FFA respectively), did not significantly correlate $(q<0.25)$ with the abundance of bacterial groups at the genus like level in either cohort. In addition, we determined non-linear multivariate relationships between the microbiota composition and tissue-specific insulin sensitivity. To this end, we ordered each dataset in quartiles of insulin sensitivity (Rd, \%EGP and \%FFA) and used random forest classifiers to determine whether the bacterial composition was related to these markers of tissue-specific insulin sensitivity, by using the highest and lowest quartiles as classes. Both cohorts showed random classification for peripheral, liver and adipose tissue insulin sensitivity, indicating no significant relationship between microbial profiles and tissue-specific insulin sensitivity (data not shown).

\section{Other measures of glucose homeostasis and insulin sensitivity}

Except for the correlation of Peptococcus niger et rel. with HbA1c ( Spearman $\rho=0.57, p=$ $5.52 \mathrm{E}-05, \mathrm{q}=0.06)$ in the MAA cohort, no significant correlation $(\mathrm{q}<0.25)$ was found 
between the abundance of specific microbial groups and HOMA-IR, HOMA-B\%, fasting glucose or insulin concentrations in either of the two cohorts (Figure 3). Correspondingly, we did not find a significant correlation $(\mathrm{q}<0.25)$ between BMI, waist circumference and abundance of genus-level bacterial groups in this study. Although almost none of the correlations were significant, it is worthy to note that the pattern of correlation of specific taxa with host variables was distinctly different in both cohorts (Figure 3).

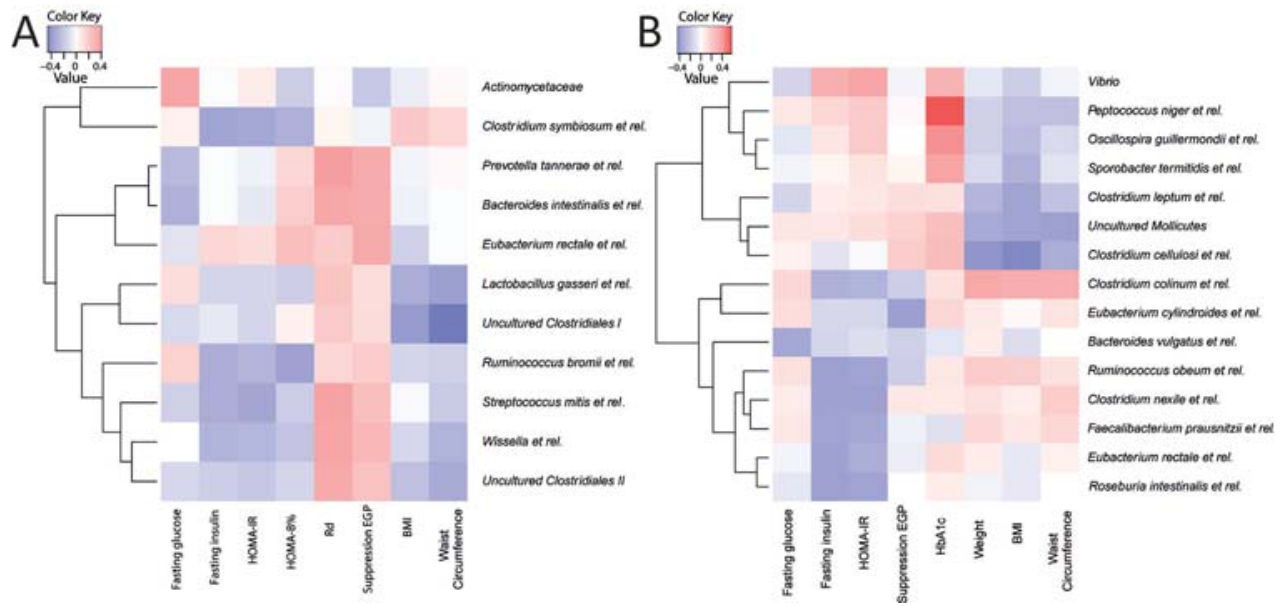

Figure 3. Correlation-heatmaps of host metabolic parameters and microbiota abundance. Heatmaps of Spearman correlations of host metabolic parameters with individual genus like bacterial groups for AMS (A) and MAA (B). Blue show negative correlations and red positive. Only the positive correlation of Peptococcus niger et rel. with HbA1c within the MAA (A) cohort remained significant after correction for multiple testing. Only correlations with Spearman $\rho>0.35$ and $p<0.25$ (uncorrected) are shown.

Furthermore, we ordered each dataset in quartiles of fasting glucose and insulin, HOMA-IR and HOMA-B $\%$ and used random forest classifiers to determine whether the bacterial composition was related to these markers, by using the highest and lowest quartiles as classes. Only MAA showed a moderate improvement over random classification for fasting glucose concentrations and HbAlc (AUC $=0.65$ ) with taxa from Clostridium clusters IV (Faecalibacterium prausnitzii et rel. and Butyrivibrio crossatus et rel.) and XIVa (Roseburia intestinalis, Clostridium nexile and Eubacterium rectale and related species) and several Proteobacteria (Enterobacter aerogenes et rel. and Vibrio) as the most important microbiota prediction features for classification of individuals with high or low fasting glucose concentrations. For HbAlc similar bacterial orders were among the most important taxa. However, different members of these higher phylogenetic groups were identified, such as for Clostridium clusters IV (Oscillospira guillermondii et rel., Sporobacter termitidis et rel., Faecalibacterium prausnitzii et rel.) and IX (Peptococcus niger et rel.) as well as Proteobacteria (Novosphingobium, Vibrio and Aeromonas). 
A

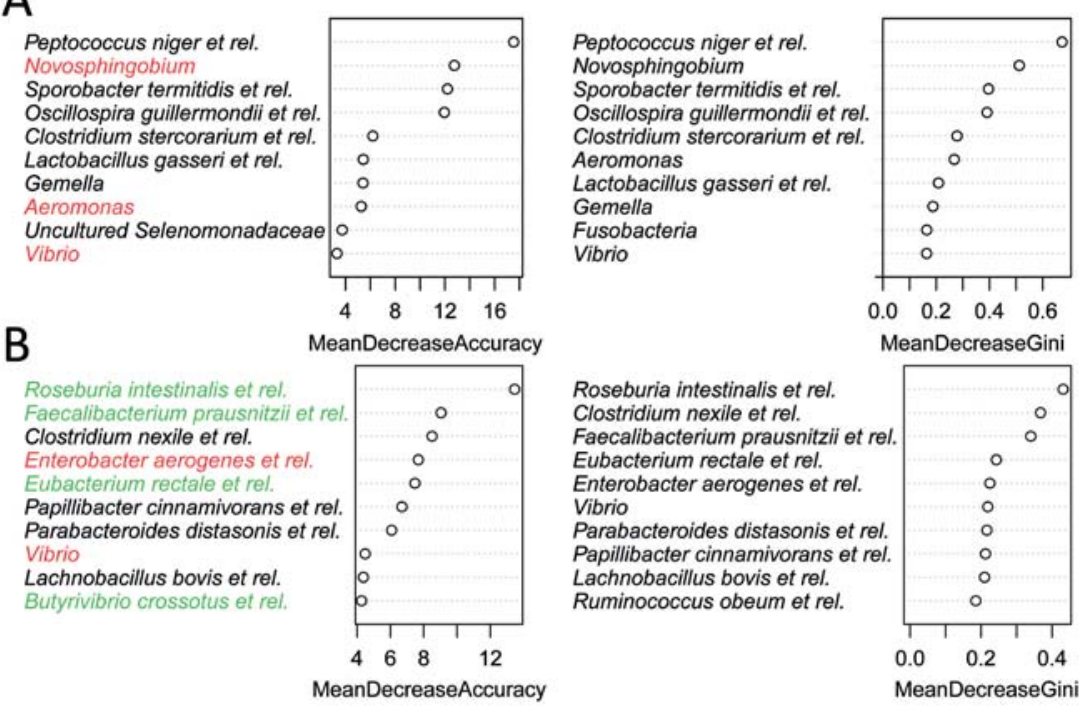

Figure 4. Top ten genus level groups with predictive power in classifying patients from MAA into the lowest and highest quartile of HbA1c (A) and fasting glucose (B). The higher the group the more the prediction power will be reduced when the specific group is removed from the Random Forests model. Taxa in red belong to the phylum Proteobacteria and green are butyrate producing Firmicutes.

\section{Discussion}

The aim of this cross-sectional study was to determine the relation between microbiota composition and tissue-specific insulin-sensitivity in two independent cohorts of obese males with ranging levels of insulin resistance. To our knowledge, this is the first observational study that takes into account hyperinsulinemic-euglycemic clamp-derived data. In two independent cohorts of obese and overweight subjects, we assessed peripheral, hepatic and adipose tissue insulin sensitivity using a two-step hyperinsulinemic-euglycemic clamp in combination with a $\left[6,6-{ }^{2} \mathrm{H}_{2}\right]$-glucose tracer infusion. In neither cohort we found a clear association between a specific fecal microbial pattern and adipose tissue, liver or skeletal muscle insulin sensitivity, indicating that predicting the role of microbiota based on baseline compositional data in host insulin sensitivity is challenging when the obese state has already developed. In line with this, almost none of the correlations between surrogate measures of insulin sensitivity and glycemic control and gut microbiota composition were significant in either of the two cohorts. In MAA, we found a relationship between Peptococcus niger et rel. with HbA1c (Spearman $\rho=0.57, p=5.52 \mathrm{E}-05, \mathrm{q}=0.06$ ). Because of this single significant correlation, we analyzed the underlying correlation matrix to see whether we could identify a similar pattern and observe conserved associations between bacterial abundance and markers of tissue-specific insulin sensitivity (e.g. consistent identical direction of a correlation between a group with a metabolic parameter in both 
cohorts), however, both cohorts showed highly divergent patterns (Figure 3). For instance Eubacterium rectale et rel. -a well-known butyrate producer-, showed conflicting correlations with fasting insulin and HOMA-IR in both cohorts, again highlighting the challenges associated with baseline observations in a single cohort.

To gain more insight into these differing patterns we applied random forest classification of quartiled host parameters. Random Forests is a supervised machine learning technique which can utilize nonlinear relationships and complex dependencies between genus-like groups to identify bacterial taxa that differentiate the faecal community composition of individuals that are in the highest or lowest range of each host parameter, respectively. The measure of the success of the method is its ability to classify samples correctly. Random classification (always choosing 1 class would yield a classification error of 0.5 , therefore, the performance should be higher if the input values or predictors (relative abundance of bacterial groups) assist in classification. Overall, in both cohorts the microbiota composition at genus level showed performance that was close to random classification, with differences in predictors as well as classification accuracy between these cohorts. To this end, only MAA showed a moderate improvement over random classification for individuals with the highest and lowest $25 \%$ of fasting glucose concentrations $(\mathrm{AUC}=0.66)$ or $\mathrm{HbAc1} \quad(\mathrm{AUC}=0.65)$. Among the matching microbial features important for classification of both parameters- were different Proteobacteria such as Vibrio (Figure 4). Other taxa that helped in the prediction of distinct fasting glucose levels were Faecalibacterium prausnitzii, Roseburia intestinalis, Clostridium nexile and Eubacterium rectale and related species. For HbAc1 these taxa were Oscillospira guillermondii, Sporobacter termitidis and Lactobacillus gasseri and related species. The most important microbiological feature for the prediction of $\mathrm{HbAc1}$ in MAA was the abundance of Peptococcus niger et rel. whose association was also identified using linear regression, underscoring the need for complementary analytical approaches. Among the above-listed identified taxa were several that are known to produce butyrate ${ }^{46}$.

This is in line with previous studies that showed an increased abundance of some universal butyrate-producing bacteria and an increase in Lactobacillus and various opportunistic pathogens, associated with T2DM in a cohort of European women ${ }^{182,314}$ and Chinese individuals ${ }^{195}$. More importantly these authors also showed that the discriminant metagenomic markers for T2D differed between the cohorts and concluded that, metagenomic predictive tools for T2D should be specific for the age and geographical location of the populations studied ${ }^{314}$. The obvious differences in observations between the cohorts and the fact that the identified associations of fasting glucose concentrations or $\mathrm{HbAcl}$ with specific gut microbiota members were only observed in one of the two cohorts illustrate that findings may differ from one cohort to another and confirm the observations by Karlsson and co-authors ${ }^{314}$. 
Remarkably, in both cohorts no relationship between microbial composition and tissuespecific insulin sensitivity, as determined by state-of-the-art clamp techniques, was detected although previous studies have indicated a differential microbial composition in T2DM or impaired glucose tolerant subjects ${ }^{182,195,314,322}$.

On top of these observations, there were striking differences between the microbiota compositions of both cohorts. Compared to AMS, the abundance of bifidobacteria was higher MAA, which has been linked to a healthier phenotype, a reduction in inflammatory markers and an improvement in glucose homeostasis and lipid metabolism ${ }^{323,}$, 324. Another difference between both cohorts was a lower abundance of specific taxa belonging to Clostridium cluster XIVa in MAA, which included known butyrate producers (e.g. Butyrivibrio crossotus and Clostridium symbiosum and related species) ${ }^{46}$. Also, there were several slight differences in metabolic profiles between both cohorts, with lower TAG and glucose concentrations as well as a reduced HOMA-B\% in MAA. Although lower abundances of butyrate-producing bacteria have previously been reported in T2DM subjects in various states of insulin resistance ${ }^{314}$, these compositional and metabolic differences were not linked in a significant way.

The strikingly cohort-specific observations suggest that the relation between microbiota composition and T2DM as well as other characteristics of the metabolic syndrome is very dependent on the selected cohort of patients and their respective baseline microbiota composition. Similar observation have been made by other researchers as well ${ }^{47,314}$. In addition, it could be that differences in microbiota composition are not associated with the insulin resistance phenotype when the overweight and/or obese state of the patient is already established, as is the case for our metabolic syndrome patients. In the latter case we cannot exclude that the composition of the fecal microbiota may play a role in the worsening of insulin sensitivity in an early stage in the development from a lean towards an overweight/obese phenotype.

With regards to the difference in composition between the cohorts, the human microbiota is highly individual, which we also clearly observed in our PCA plot (Figure 2). Although we know its composition is impacted by numerous external as well as host-specific factors, including diet, age, antibiotics use, BMI, gender, and genotype ${ }^{31}$, the interplay of variables that give rise to the variation of the microbiota is not yet fully understood because of its complexity and the influence of numerous stochastic variables, such as common exposures over a timeframe of years ${ }^{31}$. Nevertheless, the multitude of these variations and their combinations may explain why individual-specific (dysbiotic) microbiota profiles are continuously observed.

This individuality of the microbiota composition may also explain why fecal transplantation from lean donors to recipients with the metabolic syndrome slightly improved peripheral insulin sensitivity only in a subgroup of subjects (responders), whereas other individuals 
did not show any effect on these parameters upon the intervention ${ }^{90}$. It is evident that further studies are required to investigate the relation between fecal microbiota composition and insulin sensitivity in phenotypes varying in adiposity and insulin sensitivity. Such studies should also address microbial function to reveal if a microbial- or host component, or both can be found with predictive power.

Currently, we have no clear explanation of the remarkable difference between the two cohorts with respect to microbiota composition as well as the associations between microbial profiles and host metabolic parameters. Both cohorts included subjects with an increased risk for type 2 diabetes mellitus and/or metabolic syndrome in geographically closely located areas in the Netherlands ( $\sim 150 \mathrm{~km}$ distance). It is striking that the general microbiota compositional variation of MAA only partly overlapped with AMS and that a subgroup-specific microbiota was only observed in MAA. We hypothesize that a subset of the metabolic syndrome patients of this cohort exhibit an alternative state of microbiota composition that is driven by a yet unknown force which is only present in this cohort. Nevertheless, this study clearly demonstrates that cohort-specific microbiota differences hamper finding a consensus biological interpretation between studies based on single baseline cohort observations. This, combined with the complexity of individual disease pathogenesis, as well as the individual-specific differences in microbiota composition, may explain the inconsistency in observations between different studies concerning the identification of signature microbes for obesity, as well as other disorders and diseases including inflammatory bowel disease and irritable bowel syndrome ${ }^{325-327}$.

\section{Conclusion}

In the present study, tissue-specific insulin sensitivity, at the level of the adipose tissue, liver and skeletal muscle did not correlate with the abundance of microbial genus-level groups in two cohorts of obese, insulin resistant males at baseline. With respect to the surrogate measures for insulin sensitivity and measures of glycemic control HBAlc and fasting glucose, the microbiota composition showed predictive potential, but only in one of the cohorts. The latter findings stress the importance of taking metabolic profiles, environmental, genetic and microbial variables into account in future studies. Overall, our data combining detailed information on microbial composition and the insulin sensitivity phenotype in two cohorts indicated that predictions regarding the role for gut microbiota in host tissue-specific insulin sensitivity after development of the obese, insulin resistant state are difficult to extrapolate based on baseline microbiota composition alone. 



\section{Chapter 6}

NG-Tax, a highly accurate and validated pipeline for the analysis of $16 \mathrm{~S}$ rRNA amplicons from complex biomes

Javier Ramiro-Garcia*, Gerben D. A. Hermes*, Christos Giatsis, Detmer Sipkema, Erwin G. Zoetendal, Peter J Schaap, Hauke Smidt

*These authors contributed equally to this work 


\begin{abstract}
Background:Massive high-throughput sequencing of short, hypervariable segments of the $16 \mathrm{~S}$ ribosomal RNA (rRNA) gene has transformed the methodological landscape describing microbial diversity within and across complex biomes. However, several studies have shown that the methodology rather than the biological variation is responsible for the observed sample composition and distribution. This compromises true meta-analyses, although this fact is often disregarded. Results: To facilitate true meta-analysis of microbiome studies, we developed NG-Tax, a pipeline for 16S rRNA gene amplicon sequence analysis that was validated with different mock communities and benchmarked against QIIME as the currently most frequently used pipeline. The microbial composition of 49 independently amplified mock samples was characterized by sequencing two variable 16S rRNA gene regions, V4 and V5-V6, in three separate sequencing runs on Illumina's HiSeq2000 platform. This allowed evaluating important factors of technical bias in taxonomic classification: 1) run-to-run sequencing variation, 2) PCR-error, and 3) region/ primer specific amplification bias. Despite the short read length $(\sim 140 \mathrm{nt})$ and all technical biases, the average specificity of the taxonomic assignment for the phylotypes included in the mock communities was $96 \%$. On average $99.94 \%$ and $92.02 \%$ of the reads could be assigned to at least family or genus level, respectively, while assignment to 'spurious genera' represented on average only $0.02 \%$ of the reads per sample. Analysis of $\alpha$ - and $\beta$ diversity confirmed conclusions guided by biology rather than the aforementioned methodological aspects, which was not the case when samples were analysed using QIIME.

Conclusions: Different biological outcomes are commonly observed due to 16S rRNA region-specific performance. NG-Tax demonstrated high robustness against choice of region and other technical biases associated with 16S rRNA gene amplicon sequencing studies, diminishing their impact and providing accurate qualitative and quantitative representation of the true sample composition. This will improve comparability between studies and facilitate efforts towards standardization.
\end{abstract}




\section{Background}

Recent advances in massive high-throughput, short-amplicon sequencing are revolutionizing efforts to describe microbial diversity within and across complex biomes ${ }^{91}$. Cultivation-independent whole metagenome sequencing has received increasing attention in the functional characterization of individual communities. These efforts, however, remain relatively expensive on a per sample basis, and the richer but much more unstructured information content requires complex data modelling and analysis procedures $^{6}$. Therefore targeted surveys for specific taxonomic marker genes, such as the $16 \mathrm{~S}$ ribosomal RNA (rRNA) gene ${ }^{328,329}$, remain essential in many microbial ecological studies. These surveys rely on sequencing of short, PCR amplified, hypervariable subregions rather than of the full-length gene, mostly for reasons of throughput, sequence depth and cost-efficiency.

There have been great efforts to address the accuracy and reproducibility of findings from 16S rRNA gene amplicon sequencing studies through increased levels of standardization, and software pipelines provide comprehensive protocols to analyze microbial ecology datasets. However, these efforts have arguably enhanced replicability rather than reproducibility, by providing widely adopted defaults ${ }^{330}$. To this end, Drummond ${ }^{331}$ suggested that exact replication of an experiment (i.e., replicability) is less informative (although a necessary pre-requisite for any scientific endeavour) than the corroboration of findings by reproduction in different independent setups (i.e., reproducibility) ${ }^{332}$, because biological findings that are robust to independent methodologies are arguably more dependable than any single-track analysis ${ }^{330}$. This distinction is highly relevant for the field of microbial ecology, where replicability is often confused with reproducibility, which is apparent from many often non-interchangeable methodologies.

Accuracy can typically be evaluated by the addition of positive controls. Generally these are synthetic or mock communities (MCs) consisting of phylotypes that, ideally, are representative of the ecosystem of interest. MCs allow researchers to answer two essential questions concerning accuracy. 1) Do I retrieve the number of species I put in, and if so are they correctly assigned? 2) How well does the sequencing and data analysis procedure reproduce species relative abundances?

Reproducibility can be evaluated by comparing separate sequencing runs and different primer pairs that cover distinct $16 \mathrm{~S}$ rRNA gene regions. Although replicability is often achieved, accuracy has been shown to be challenging especially at higher taxonomic resolution such as at genus level ${ }^{128,333}$.

Central to all 16S rRNA gene amplicon studies are Operational Taxonomic Units (OTUs). These are often regarded as the synthetic proxy for microbial species and are typically clustered at $97 \%$ sequence similarity. However, the prokaryotic species definition remains a 
hotly debated topic without any satisfying solution so $\operatorname{far}^{116,117,139}$. Moreover, the $97 \%$ sequence similarity threshold is based on the complete 16S rRNA gene $(\sim 1500 \mathrm{nt})$, and although sequence variability is not evenly distributed it is routinely applied to short reads of 100-500 nt. Different regions would therefore require their own species level cut-off. This combination of an ambiguous prokaryotic species definition and its application to short reads, is the foundation for many complications regarding 'correct' OTU clustering. Hence there is little consensus on key experimental choices such as primers, targeted variable regions and OTU picking/clustering algorithms. Each of these technical aspects generate biases, and different methods produce clearly distinct results, leading to a situation where results of current studies cannot be easily compared or extrapolated to other study designs. Therefore there is a strong need for standardization.

Historically, 16S rRNA gene sequences generated in a project were initially clustered de novo into OTUs at $>97 \%$ sequence similarity using various clustering algorithms, mostly because available 16S rRNA gene reference databases were thought to provide insufficient coverage $^{134,334-336}$. Although new clustering algorithms that reduce the influence of clustering parameters, such as a hard cutoff for cluster similarity, have been specifically developed for amplicons ${ }^{337}$, cluster generation is context-dependent, i.e. different datasets generate different clusters, and different algorithms may produce different end-results ${ }^{140,330}$. Therefore, even though the same analysis framework is used, independent studies remain incomparable at OTU level. Consequently, reference-based OTU clustering has received increasing attention, due to the need for standardization, and because de-novo OTU clustering for very large datasets, such as those generated by Hiseq and Miseq sequencers has become computationally very intensive, unless greedy heuristics are employed which suffer from the problems described above. With reference-based OTU clustering, sequences are mapped to pre-clustered reference sets of curated 16S rRNA gene sequences, provided by dedicated databases such as the Ribosomal Database Project (RDP), Greengenes and SILVA $^{111,338,339}$. The consequence of this approach is that the 'quality' of the clustering of the reference set propagates to reference-picked OTUs. Clustering has limited robustness ${ }^{140}$, 330,340 , and unbalances in databases due to over- or under-representation of certain species as well as error hotspots that are not necessarily matched to the variable regions ${ }^{151}$, can potentially lead to a biased cluster formation, driven by non-biological factors. These effects have been previously ignored or underestimated in reference OTU picking protocols $^{330}$.

Another essential experimental choice concerns the selection of targeted variable region, because it should represent the sequence variability encountered with the full-length gene. Despite several studies comparing the performance of diverse regions, sequence lengths, sequencing platforms and taxon assignment methodologies, both within and across laboratories ${ }^{122,127,143,151,341-343}$, there still is no fully consensus of best choices for variable regions even if some initiatives like the Earth Microbiome Project ${ }^{344}$ are setting some 
standards. There are several factors that can lead to the commonly observed highly regionspecific behaviour across datasets: 1) PCR bias of varying degrees ${ }^{127,151,345}, 2$ ) different regions are associated with different error profiles and different rates of chimera formation ${ }^{151,346}$, and 3) the actual variation contained in the sequence is dissimilar (e.g. some regions are not variable enough to differentiate between genera, while others are), which in turn can affect clustering ${ }^{330}$.

Apart from the use of a diverse range of primers and OTU picking protocols that can cause differences in results between studies and/or laboratories, sequencing error is a third important factor that defines data quality. Massive high throughput, short read length sequencing platforms have not been developed for amplicon sequencing but rather for whole genome sequencing, where sequence errors in individual reads is less important. However, in $16 \mathrm{~S}$ rRNA gene amplicon sequencing every sequencing error could potentially lead to the false discovery of a new biomarker. To avoid overestimation of microbial diversity, stringent quality filtering is therefore considered essential ${ }^{333}$.

Methodology rather than biology has often been shown to be the largest driver of variation in microbiome studies ${ }^{127,140,151,330,343,347-349}$, and this aspect of amplicon sequencing is increasingly addressed in literature. Nevertheless, a satisfactory solution has not been found. To address the aforementioned challenges we have applied several recommendations from literature to validate high throughput, high-resolution microbiota profiling, using Illumina Hiseq2000 101nt paired end sequencing data as a test case. We implemented redundancy by sequencing two tandem variable 16S rRNA gene regions in parallel (V4 and V5-V6). To find optimal filtering settings and to empirically determine the noise floor, multiple standardized mock communities specifically designed to tackle issues associated with filtering parameter optimization were added to each sequencing run. To our knowledge a similar setup with multiple MCs and different variable regions has not been applied to datasets generated by the Illumina platform. This set-up has enabled us to develop NG-Tax, a pipeline that accounts for error associated with a range of technical aspects of 16S rRNA gene amplicon sequencing. NG-Tax will improve comparability by removing technical bias and facilitate efforts towards standardization, by focusing on reproducibility as well as accuracy. To assess the performance we benchmarked the results obtained with NG-Tax with results obtained with QIIME ${ }^{350}$, a common pipeline used for the analysis of this type of data. 


\section{Results and discussion}

\section{NG-Tax layout}

NG-Tax consists of three core elements, namely barcode-primer filtering, OTU-picking and taxonomic assignment (Figure 1). User examples and details of each step of the pipeline can be found in the user manual in Dataset 1.

\section{Barcode-Primer filtering}

In a first step, paired end libraries are combined, and only read pairs with perfectly matching primers and barcodes are retained. To this end, both primers are barcoded to facilitate identification of chimeras produced during library generation after pooling of individual PCR products.

\section{OTU picking}

For each sample an OTU table is created with the most abundant sequences, using a minimum user defined relative abundance threshold. In this particular study we employed a threshold of $0.1 \%$ minimum relative abundance. Lowering the threshold will lead to the acceptance of low abundant OTUs, with an increased probability of these OTUs being artifacts due to sequencing and PCR errors. Abundance thresholds are commonly used to remove spurious OTUs generated by sequencing and PCR errors ${ }^{128,351}$, but previous studies applied a fraction threshold defined by the complete dataset under study, thereby ignoring sample size heterogeneity which may lead to under-representation of asymmetrically distributed OTUs.

Commonly employed quality filtering parameters based on Phred score, such as minimum average Phred score, maximum number of ambiguous positions, maximum bad run length, trimming and minimum read length after quality trimming, are not utilized in NG-Tax because quality scores from the Illumina base caller have been shown to be of limited use for the identification of actual sequence errors for $16 \mathrm{~S}$ rRNA gene amplicon studies ${ }^{333,352}$. Additionally, these quality scores only check for errors that occurred during sequencing, but do not account for other sources of error, such as PCR amplification, whereas quality filtering by abundance is sensitive to any source of error. Moreover, the application of global parameters (e.g. average Phred score) ignores that error is sequence-specific, and hence some sequences could be affected more than others. If a species specific amplicon is more prone to PCR or sequencing errors, the relative abundance of that particular species will be underestimated. To compensate for this potential bias, discarded reads are clustered to the OTUs with one mismatch.

Finally, all OTUs are subjected to non-reference based chimera checking according to the following principle: given three OTUs named A, B and C, C will be considered a chimera 
when the following conditions are satisfied: $\mathrm{C}$ and $\mathrm{A} 5^{\prime}$ reads are identical, $\mathrm{C}$ and $\mathrm{B}$ 3' reads are identical and both OTUs, A and B, are at least twice as abundant as OTU C.

\section{Taxonomic assignment}

In the current version of NG-Tax, taxonomy is assigned to OTUs utilizing the usearch algorithm $^{336}$ and the Silva_111_SSU Ref database, containing 731,863 unique full length $16 \mathrm{~S}$ rRNA gene sequences. To ensure maximum resolution and avoid the risk of errors due to clustering-associated flaws (e.g. reference sequence error hotspots, overrepresentation of certain species and lack of robustness in cluster formation by clustering algorithms), we use the non-clustered database. To speed up the procedure by several orders of magnitude, $16 \mathrm{~S}$ rRNA gene sequences from the reference database are trimmed to contain only the region amplified by the primers. For each OTU, a taxonomic assignment is retrieved at six different identity thresholds levels $(100 \%, 98 \%, 97 \%, 95 \%, 92 \%$ and $90 \%)$ and at two taxonomic levels (genus and family). The final taxonomic label is determined by the assignments that show concordance at the highest taxonomic resolution.

NG-Tax layout

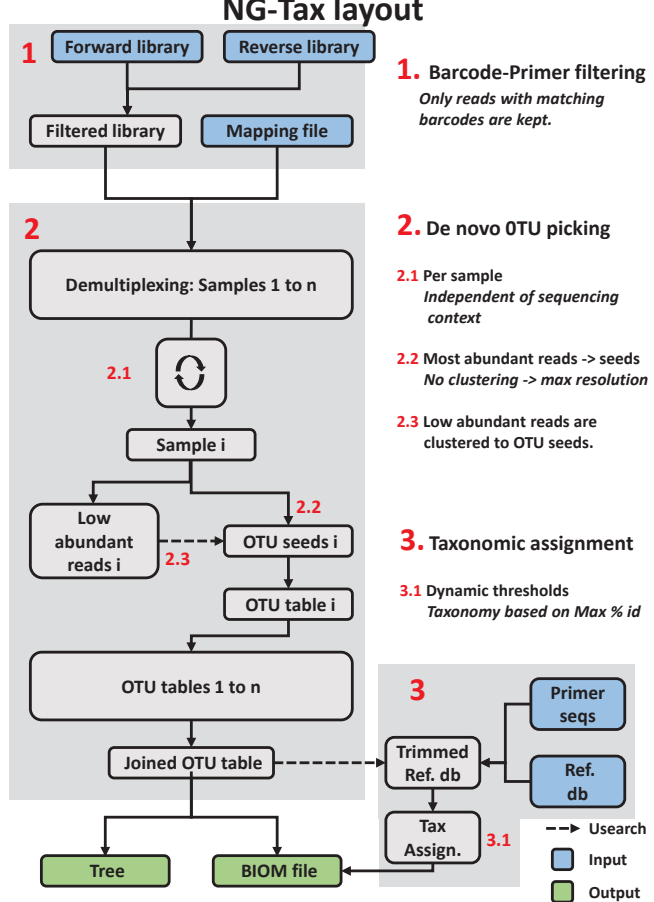

Figure 1. NG-Tax layout. Input files are depicted in blue, output files are depicted in green and clustering processes using usearch are indicated with dashed lines. Details for some steps of the pipeline are marked with red numbers. 


\section{Validation}

\section{Datasets}

Our main objective was to develop a pipeline that accurately reproduces the synthetic MCs and also reduces the impact of experimental choices on the results. To achieve this goal, four synthetic communities of varying complexity were created, consisting of 16S rRNA gene amplicons of phylotypes (PTs) associated with the human GI-tract (Table 1). This specific setup limited the likelihood of overfitting to a particular OTU composition or distribution and allowed us to assess (1) the quantification potential, (2) noise floor and (3) the effect of richness and diversity on quality filtering parameters, thus ensuring a higher fidelity with biological samples than by using a single MC. As a reference, to assess the quality of the taxonomic classifications, full length sequences for all PTs were obtained through Sanger sequencing. Expected MCs were created by trimming the full length sequences to the sequenced region. $\mathrm{MC} 1$ and MC2 consisted of equimolar amounts of 17 and 55 PTs, respectively. MC3 contained 55 PTs in staggered concentrations typical for the human GI-tract, and MC4 included 50 PTs with relative abundances ranging between 0.001 and $2.49 \%$. To account for pipetting errors, each of the four MCs was produced in triplicate. To design a pipeline that puts more focus on biology, these $12 \mathrm{MC}$ templates were used to sequence the MCs with different conditions that cover most of the technical bias associated with 16S rRNA gene amplicon studies reported in literature. To this end, we 1) targeted either region V4 or region V5-V6, 2) used four PCR protocols differing in the number of PCR cycles and reaction volumes 3) PCR products were analysed in three different sequencing runs and in seven different libraries, and 4) two different library preparation protocols (with and without an extra amplification of 10 cycles) were applied (Table 2). In addition the sequencing depth ranged from 2363 to 335822 reads per sample. 


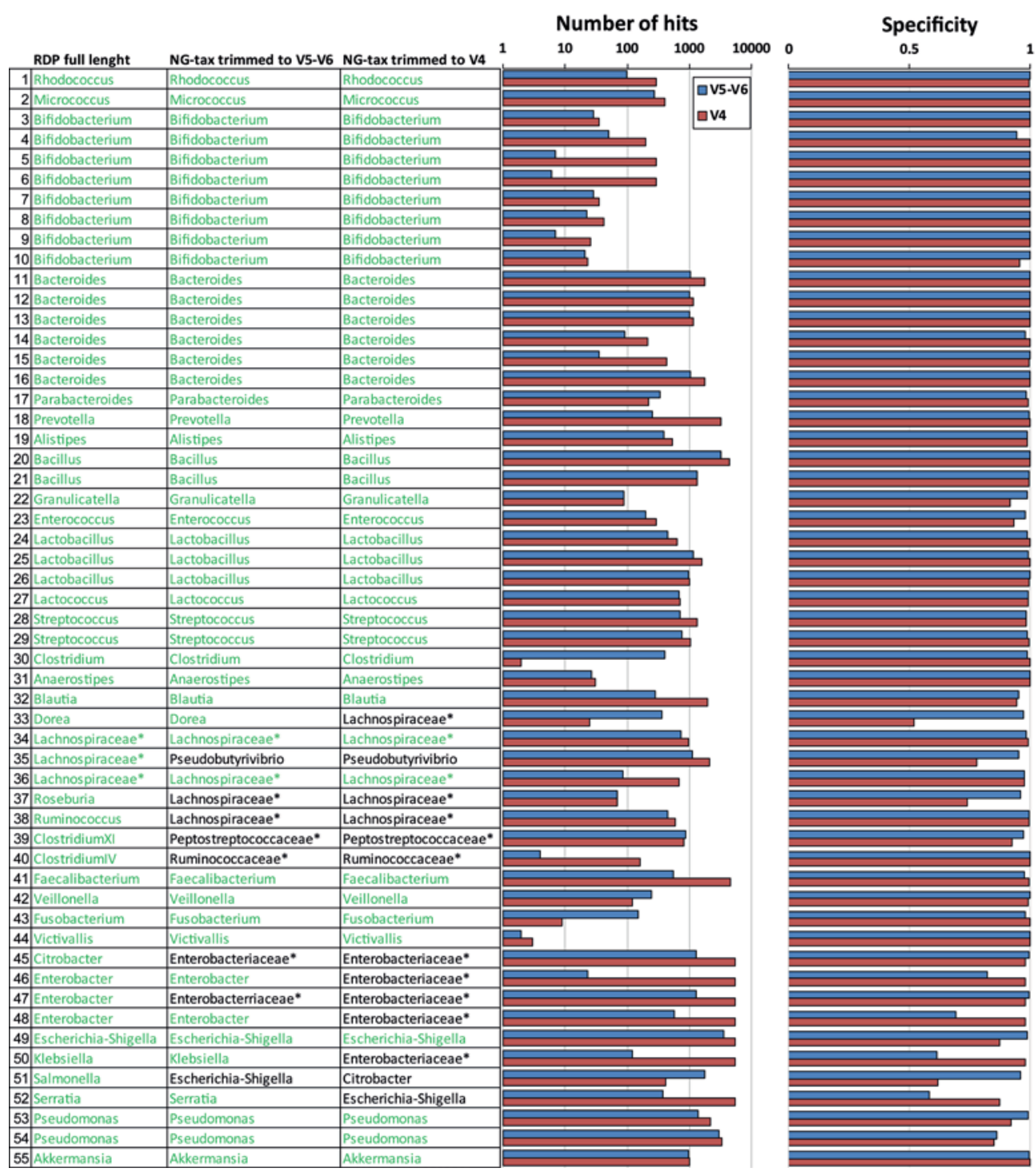

Figure 2. NG-Tax Assignment quality of the 55 MC phylotypes. Three taxonomic assignments are shown: RDP full length, NGTax V5-V6 trimmed, and NG-Tax V4 trimmed. If NG-Tax assignments are in agreement with RDP full length assignment, that classification is shown in green. Assignment specificity (the fraction of hits with an identical label) and the total number of hits supporting this taxonomic label are shown in blue (V5-V6) and red (V4) 
Table 1. Composition of the four MCs. Relative abundance and taxonomy of 55 reference sequences used in this study. The taxonomic label was based on classification of the full length reference sequences according to the RDP classifier.

\begin{tabular}{|c|c|c|c|c|c|c|c|c|c|c|}
\hline \multicolumn{7}{|c|}{ RDP Naive Bayesian rRNA Classifier Version 2.10, October 2014} & \multirow[t]{2}{*}{ MC } & \multirow{2}{*}{$\begin{array}{l}\text { MC } \\
1.82\end{array}$} & \multirow{2}{*}{$\begin{array}{l}\text { MC } \\
1.1\end{array}$} & \multirow{2}{*}{$\begin{array}{c}\text { MC } \\
0.001\end{array}$} \\
\hline 1 & Bacte & Actinobacteria & Actinobacteria & Actinomycetales & Nocardiaceae & Rhodococcus & & & & \\
\hline 2 & Bacte & Actinobacteria & Actinobacteria & Actinomycetales & Micrococcaceae & Micrococcus & & 1.82 & 1.1 & 0.01 \\
\hline 3 & Bacte & Actinobacteria & Actinobacteria & Bifidobacteriales & Bifidobacteriaceae & Bifidobacterium & 5.8 & 1.82 & 1.1 & 0.10 \\
\hline 4 & Bacte & Actinobacteria & Actinobacteria & Bifidobacteriales & Bifidobacteriaceae & Bifidobacterium & & 1.82 & 1.1 & 2.49 \\
\hline 5 & Bacte & Actinobacteria & Actinobacteria & Bifidobacteriales & Bifidobacteriaceae & Bifidobacterium & & 1.82 & 1.1 & \\
\hline 6 & Bacte & Actinobacteria & Actinobacteria & Bifidobacteriales & Bifidobacteriaceae & Bifidobacterium & & 1.82 & 1.1 & \\
\hline 7 & Bacte & Actinobacteria & Actinobacteria & Bifidobacteriales & Bifidobacteriaceae & Bifidobacterium & & 1.82 & 1.1 & \\
\hline 8 & Bacte & Actinobacteria & Actinobacteria & Bifidobacteriales & Bifidobacteriaceae & Bifidobacterium & & 1.82 & 1.1 & \\
\hline 9 & Bacte & Actinobacteria & Actinobacteria & Bifidobacteriales & Bifidobacteriaceae & Bifidobacterium & & 1.82 & 1.1 & \\
\hline 1 & Bacte & Actinobacteria & Actinobacteria & Bifidobacteriales & Bifidobacteriaceae & Bifidobacterium & & 1.82 & 1.1 & \\
\hline 1 & Bacte & Bacteroidetes & Bacteroidia & Bacteroidales & Bacteroidaceae & Bacteroides & & 1.82 & 1.1 & 2.49 \\
\hline 1 & Bacte & Bacteroidetes & Bacteroidia & Bacteroidales & Bacteroidaceae & Bacteroides & & 1.82 & 1.1 & 2.49 \\
\hline 1 & Bacte & Bacteroidetes & Bacteroidia & Bacteroidales & Bacteroidaceae & Bacteroides & & 1.82 & 1.1 & 2.49 \\
\hline 1 & Bacte & Bacteroidetes & Bacteroidia & Bacteroidales & Bacteroidaceae & Bacteroides & & 1.82 & 1.1 & 2.49 \\
\hline 1 & Bacte & Bacteroidetes & Bacteroidia & Bacteroidales & Bacteroidaceae & Bacteroides & & 1.82 & 4.0 & 2.49 \\
\hline 1 & Bacte & Bacteroidetes & Bacteroidia & Bacteroidales & Bacteroidaceae & Bacteroides & 5.8 & 1.82 & 1.1 & 2.49 \\
\hline 1 & Bacte & Bacteroidetes & Bacteroidia & Bacteroidales & Porphyromonadacea & Parabacteroides & 5.8 & 1.82 & 4.6 & 0.001 \\
\hline 1 & Bacte & Bacteroidetes & Bacteroidia & Bacteroidales & Prevotellaceae & Prevotella & 5.8 & 1.82 & $i .1$ & 0.10 \\
\hline 1 & Bacte & Bacteroidetes & Bacteroidia & Bacteroidales & Rikenellaceae & Alistipes & 5.8 & 1.82 & 8.1 & 0.01 \\
\hline 2 & Bacte & Firmicutes & Bacilli & Bacillales & Bacillaceae 1 & Bacillus & & 1.82 & 1.1 & 2.49 \\
\hline 2 & Bacte & Firmicutes & Bacilli & Bacillales & Bacillaceae 1 & Bacillus & & 1.82 & 1.1 & 2.49 \\
\hline 2 & Bacte & Firmicutes & Bacilli & Lactobacillales & Carnobacteriaceae & Granulicatella & & 1.82 & 1.1 & 2.49 \\
\hline 2 & Bacte & Firmicutes & Bacilli & Lactobacillales & Enterococcaceae & Enterococcus & & 1.82 & 1.1 & 2.49 \\
\hline 2 & Bacte & Firmicutes & Bacilli & Lactobacillales & Lactobacillaceae & Lactobacillus & & 1.82 & 1.1 & 2.49 \\
\hline 2 & Bacte & Firmicutes & Bacilli & Lactobacillales & Lactobacillaceae & Lactobacillus & & 1.82 & 1.1 & 2.49 \\
\hline 2 & Bacte & Firmicutes & Bacilli & Lactobacillales & Lactobacillaceae & Lactobacillus & & 1.82 & 1.1 & 2.49 \\
\hline 2 & Bacte & Firmicutes & Bacilli & Lactobacillales & Streptococcaceae & Lactococcus & & 1.82 & 1.1 & 2.49 \\
\hline 2 & Bacte & Firmicutes & Bacilli & Lactobacillales & Streptococcaceae & Streptococcus & 5.8 & 1.82 & 1.1 & 2.49 \\
\hline 2 & Bacte & Firmicutes & Bacilli & Lactobacillales & Streptococcaceae & Streptococcus & & 1.82 & 1.1 & 2.49 \\
\hline 3 & Bacte & Firmicutes & Clostridia & Clostridiales & Clostridiaceae 1 & Clostridium sensu stricto & 5.8 & 1.82 & 1.1 & 2.49 \\
\hline 3 & Bacte & Firmicutes & Clostridia & Clostridiales & Lachnospiraceae & Anaerostipes & 5.8 & 1.82 & 1.1 & 2.49 \\
\hline 3 & Bacte & Firmicutes & Clostridia & Clostridiales & Lachnospiraceae & Blautia & 5.8 & 1.82 & 5.8 & $\overline{0.001}$ \\
\hline 3 & Bacte & Firmicutes & Clostridia & Clostridiales & Lachnospiraceae & Dorea & 5.8 & 1.82 & 1.1 & 2.49 \\
\hline 3 & Bacte & Firmicutes & Clostridia & Clostridiales & Lachnospiraceae & Lachnospiracea incertae $s$ & 5.8 & 1.82 & 1.1 & 2.49 \\
\hline 3 & Bacte & Firmicutes & Clostridia & Clostridiales & Lachnospiraceae & Lachnospiracea_incertae_s & 5.8 & 1.82 & 1.1 & 2.49 \\
\hline 3 & Bacte & Firmicutes & Clostridia & Clostridiales & Lachnospiraceae & Lachnospiracea_incertae_s & & 1.82 & 1.1 & 2.49 \\
\hline 3 & Bacte & Firmicutes & Clostridia & Clostridiales & Lachnospiraceae & Roseburia & 5.8 & 1.82 & 8.7 & 0.01 \\
\hline 3 & Bacte & Firmicutes & Clostridia & Clostridiales & Lachnospiraceae & Ruminococcus2 & & 1.82 & 1.1 & 2.49 \\
\hline 3 & Bacte & Firmicutes & Clostridia & Clostridiales & Peptostreptococcace & Clostridium XI & 5.8 & 1.82 & 1.1 & 2.49 \\
\hline 4 & Bacte & Firmicutes & Clostridia & Clostridiales & Ruminococcaceae & Clostridium IV & & 1.82 & 1.1 & 2.49 \\
\hline 4 & Bacte & Firmicutes & Clostridia & Clostridiales & Ruminococcaceae & Faecalibacterium & 5.8 & 1.82 & 6.9 & 0.10 \\
\hline 4 & Bacte & Firmicutes & Negativicutes & Selenomonadales & Veillonellaceae & Veillonella & & 1.82 & 1.1 & 2.49 \\
\hline 4 & Bacte & Fusobacteria & Fusobacteriia & Fusobacteriales & Fusobacteriaceae & Fusobacterium & & 1.82 & 1.7 & 2.49 \\
\hline 4 & Bacte & Lentisphaerae & Lentisphaeria & Victivallales & Victivallaceae & Victivallis & & 1.82 & 0.5 & 2.49 \\
\hline 4 & Bacte & Proteobacteria & Gammaproteobacter & Enterobacteriales & Enterobacteriaceae & Citrobacter & & 1.82 & 1.1 & 2.49 \\
\hline 4 & Bacte & Proteobacteria & Gammaproteobacter & Enterobacteriales & Enterobacteriaceae & Enterobacter & & 1.82 & 1.1 & 2.49 \\
\hline 4 & Bacte & Proteobacteria & Gammaproteobacter & Enterobacteriales & Enterobacteriaceae & Enterobacter & & 1.82 & 1.1 & 2.49 \\
\hline 4 & Bacte & Proteobacteria & Gammaproteobacter & Enterobacteriales & Enterobacteriaceae & Enterobacter & & 1.82 & 1.1 & 2.49 \\
\hline 4 & Bacte & Proteobacteria & Gammaproteobacter & Enterobacteriales & Enterobacteriaceae & Escherichia/Shigella & 5.8 & 1.82 & 4.0 & 2.49 \\
\hline 5 & Bacte & Proteobacteria & Gammaproteobacter & Enterobacteriales & Enterobacteriaceae & Klebsiella & & 1.82 & 1.1 & 2.49 \\
\hline 5 & Bacte & Proteobacteria & Gammaproteobacter & Enterobacteriales & Enterobacteriaceae & Salmonella & & 1.82 & 1.1 & 2.49 \\
\hline 5 & Bacte & Proteobacteria & Gammaproteobacter & Enterobacteriales & Enterobacteriaceae & Serratia & & 1.82 & 1.1 & 2.49 \\
\hline 5 & Bacte & Proteobacteria & Gammaproteobacter & Pseudomonadale & Pseudomonadaceae & Pseudomonas & & 1.82 & 1.1 & 2.49 \\
\hline 5 & Bacte & Proteobacteria & Gammaproteobacter & Pseudomonadale & Pseudomonadaceae & Pseudomonas & & 1.82 & 1.1 & 2.49 \\
\hline 5 & Bacte & Verrucomicro & Verrucomicrobiae & Verrucomicrobia & Verrucomicrobiacea & Akkermansia & 5.8 & 1.82 & 2.9 & 2.49 \\
\hline
\end{tabular}


Table 2. The 49 sequenced MC samples.

\begin{tabular}{|c|c|c|c|c|c|c|c|c|c|}
\hline & Name & MC & Template & Region & Run & Library & Extra & PCR & PCR \\
\hline 1 & Mc.1.1.101 & 1 & 1.1 & V5-V6 & 1 & 1 & Yes & 25 & $3 \times 40 \mu 1$ \\
\hline 2 & Mc.1.2.101 & 1 & 1.2 & V5-V6 & 1 & 1 & Yes & 25 & $3 \times 40 \mu 1$ \\
\hline 3 & Mc.1.3.101 & 1 & 1.3 & V5-V6 & 1 & 1 & Yes & 25 & $3 \times 40 \mu 1$ \\
\hline 4 & Mc.2.1.101 & 2 & 2.1 & V5-V6 & 1 & 1 & Yes & 25 & $3 \times 40 \mu 1$ \\
\hline 5 & Mc.2.2.101 & 2 & 2.2 & V5-V6 & 1 & 1 & Yes & 25 & $3 \times 40 \mu 1$ \\
\hline 6 & Mc.2.3.101 & 2 & 2.3 & V5-V6 & 1 & 1 & Yes & 25 & $3 \times 40 \mu 1$ \\
\hline 7 & Mc.3.1.101 & 3 & 3.1 & V5-V6 & 1 & 1 & Yes & 25 & $3 \times 40 \mu 1$ \\
\hline 8 & Mc.3.2.101 & 3 & 3.2 & V5-V6 & 1 & 1 & Yes & 25 & $3 \times 40 \mu 1$ \\
\hline 9 & Mc.3.3.101 & 3 & 3.3 & V5-V6 & 1 & 1 & Yes & 25 & $3 \times 40 \mu 1$ \\
\hline 10 & Mc.4.1.101 & 4 & 4.1 & V5-V6 & 1 & 1 & Yes & 25 & $3 \times 40 \mu 1$ \\
\hline 11 & Mc.4.2.101 & 4 & 4.2 & V5-V6 & 1 & 1 & Yes & 25 & $3 \times 40 \mu 1$ \\
\hline 12 & Mc.4.3.101 & 4 & 4.3 & V5-V6 & 1 & 1 & Yes & 25 & $3 \times 40 \mu 1$ \\
\hline 13 & Mc.1.1.102 & 1 & 1.1 & V4 & 2 & 2 & No & 25 & $3 \times 40 \mu 1$ \\
\hline 14 & Mc.1.2.102 & 1 & 1.2 & V4 & 2 & 2 & No & 25 & $3 \times 40 \mu 1$ \\
\hline 15 & Mc.1.3.102 & 1 & 1.3 & V4 & 2 & 2 & No & 25 & $3 \times 40 \mu 1$ \\
\hline 16 & Mc.2.1.102 & 2 & 2.1 & V4 & 2 & 2 & No & 25 & $3 \times 40 \mu 1$ \\
\hline 17 & Mc.2.2.102 & 2 & 2.2 & V4 & 2 & 2 & No & 25 & $3 \times 40 \mu 1$ \\
\hline 18 & Mc.2.2.dup.102 & 2 & 2.2 & V4 & 2 & 2 & No & 25 & $3 \times 40 \mu 1$ \\
\hline 19 & Mc. 2.2 .30 .102 & 2 & 2.2 & V4 & 2 & 2 & No & 30 & $3 \times 40 \mu 1$ \\
\hline 20 & Mc. 2.2.35.102 & 2 & 2.2 & V4 & 2 & 2 & No & 35 & $3 \times 40 \mu 1$ \\
\hline 21 & Mc. $2.2 .1 x .102$ & 2 & 2.2 & V4 & 2 & 2 & No & 25 & $1 \times 100$ \\
\hline 22 & Mc.2.2.1x.102 & 2 & 2.2 & V4 & 2 & 2 & No & 25 & $1 \times 100$ \\
\hline 23 & Mc.2.3.102 & 2 & 2.3 & V4 & 2 & 2 & No & 25 & $3 \times 40 \mu 1$ \\
\hline 24 & Mc.3.1.102 & 3 & 3.1 & V4 & 2 & 2 & No & 25 & $3 \times 40 \mu 1$ \\
\hline 25 & Mc.3.2.102 & 3 & 3.2 & V4 & 2 & 2 & No & 25 & $3 \times 40 \mu 1$ \\
\hline 26 & Mc.3.3.102 & 3 & 3.3 & V4 & 2 & 2 & No & 25 & $3 \times 40 \mu 1$ \\
\hline 27 & Mc.4.1.102 & 4 & 4.1 & V4 & 2 & 2 & No & 25 & $3 \times 40 \mu 1$ \\
\hline 28 & Mc.4.2.102 & 4 & 4.12 & V4 & $\frac{2}{2}$ & $\frac{2}{2}$ & No & 25 & $\frac{J \lambda+0 \mu 1}{3 \times 40 \mu 1}$ \\
\hline 29 & Mc.4.3.102 & 4 & 4.3 & V4 & 2 & 2 & No & 25 & $3 \times 40 \mu 1$ \\
\hline 30 & Mc.2.1.103 & 2 & 2.1 & V4 & 3 & 3 & No & 25 & $3 \times 40 \mu 1$ \\
\hline 31 & Mc.2.2.103 & 2 & 2.2 & V4 & 3 & 3 & No & 25 & $3 \times 40 \mu 1$ \\
\hline 32 & Mc.4.1.103 & 4 & 4.1 & V4 & 3 & 3 & No & 25 & $3 \times 40 \mu 1$ \\
\hline 33 & Mc.4.2.103 & 4 & 4.2 & V4 & 3 & 3 & No & 25 & $3 \times 40 \mu 1$ \\
\hline 34 & Mc.2.1.104 & 2 & 2.1 & V4 & 3 & 4 & No & 25 & $3 \times 40 \mu 1$ \\
\hline 35 & Mc.2.2.104 & 2 & 2.2 & V4 & 3 & 4 & No & 25 & $3 \times 40 \mu 1$ \\
\hline 36 & Mc.4.1.104 & 4 & 4.1 & V4 & 3 & 4 & No & 25 & $3 \times 40 \mu 1$ \\
\hline 37 & Mc.4.2.104 & 4 & 4.2 & V4 & 3 & 4 & No & 25 & $3 \times 40 \mu 1$ \\
\hline$\frac{38}{38}$ & Mc.2.1.105 & 2 & 2.1 & V4 & 3 & $\frac{4}{5}$ & No & $\frac{25}{25}$ & $\frac{J \lambda+0 \mu 1}{3 \times 40 \mu 1}$ \\
\hline 39 & Mc.2.2.105 & 2 & 2.2 & V4 & 3 & 5 & No & 25 & $3 \times 40 \mu 1$ \\
\hline 40 & Mc.4.1.105 & 4 & 4.1 & V4 & 3 & 5 & No & 25 & $3 \times 40 \mu 1$ \\
\hline 41 & Mc.4.2.105 & 4 & 4.2 & V4 & 3 & 5 & No & 25 & $3 \times 40 \mu 1$ \\
\hline 42 & Mc.2.1.106 & 2 & 2.1 & V4 & 3 & 6 & No & 25 & $3 \times 40 \mu 1$ \\
\hline 43 & Mc.2.2.106 & 2 & 2.2 & V4 & 3 & 6 & No & 25 & $3 \times 40 \mu 1$ \\
\hline 44 & Mc.4.1.106 & 4 & 4.1 & V4 & 3 & 6 & No & 25 & $3 \times 40 \mu 1$ \\
\hline 45 & Mc.4.2.106 & 4 & 4.2 & V4 & 3 & 6 & No & 25 & $3 \times 40 \mu 1$ \\
\hline 46 & Mc.2.1.107 & 2 & 2.1 & V4 & 3 & 7 & No & 25 & $3 \times 40 \mu 1$ \\
\hline 47 & Mc.2.2.107 & 2 & 2.2 & V4 & 3 & 7 & No & 25 & $3 \times 40 \mu 1$ \\
\hline 48 & Mc.4.1.107 & 4 & 4.1 & V4 & 3 & $\frac{1}{7}$ & No & $\frac{25}{25}$ & $3 \times 40 \mu 1$ \\
\hline 49 & Mc.4.2.107 & 4 & 4.2 & V4 & 3 & 7 & No & 25 & $3 \times 40 \mu 1$ \\
\hline
\end{tabular}




\section{NG-Tax classification of short reads versus full length classification}

To evaluate the accuracy and reproducibility of taxonomic classification using a low information content of $\sim 140 \mathrm{nt}$ compared to a maximal information content of $\sim 1500 \mathrm{nt}$, we compared the NG-Tax classification of all 55 reference sequences used in this study trimmed to V4 and V5-V6, with a classification of the corresponding full length reference sequences using the RDP classifier (RDPc) $)^{122}$ (Figure 2). At family level, all three classifications (i.e. full length, V4 and V5-V6) were in complete concordance for all phylotypes. Correspondingly, the consistency at genus level was very high. Only a few phylotypes (nine and five for V4 and V5-V6 amplicons, respectively), that belong to poorly classified families such as Peptostreptococcaceae, Ruminococceae and Enterobacteriaceae, attained higher resolution using the full length sequences and RDPc. In turn, for Pseudobutyrivibrio (PT35), a higher resolution was attained with short reads due to the high specificity of the hypervariable regions, which can be overshadowed when using the full length sequence. Lastly, only two (PT51, PT52, V4) and one (PT51, V5-V6) assignments at genus level (both members of the Enterobacteriaceae) were incongruent between classification of the short and full length sequences. Overall, the V5-V6 amplicons outperformed the V4 amplicons because this region allowed for differentiation between members of the Enterobacteriaceae. The average taxonomic specificity (percentage of hits with an identical taxonomic label) for all reference phylotypes was $96 \%$ for both regions with an average of 1485 and 635 hits for regions V4 and V5-V6, respectively. The high specificity and high number of hits at very high identity thresholds, combined with the fact that the vast majority of V4 and V5-V6 based assignments matched, testifies for the reliability and quality of the assignments.

\section{Observed versus expected microbial profiles}

To assess the ability to reproduce the expected composition of the MCs we benchmarked NG-Tax with QIIME, a commonly employed 16S rRNA gene amplicon analysis pipeline. The reproduction of $\mathrm{MC}$ compositional profiles generated by amplicon sequencing on Illumina platforms commonly suffers from a high fraction of poorly classified and spurious OTUs $^{333}$. Using QIIME, up to $20 \%$ of OTUs per sample could not be assigned beyond the class level (Figure 3). In contrast, with NG-Tax we observed excellent reproduction of the expected profiles (Figure 4). An average of $92.02 \%$ of the reads could be assigned to genus level and $99.94 \%$ to at least family level. Spurious genera (Robinsoniella, Subdoligranulum, Cupriavidus, Ralstonia, Kluyvera and Pantoea) represented on average only $0.02 \%$ of the reads per sample compared to an average of $23 \%$ misclassified reads using QIIME ${ }^{9}$. 
One template, PT17 (Parabacteroides), attracted so much sequencing error in the V4 region that it was rendered undetectable although it was amplified by the primers (Supplementary Figure 1). Therefore, to test both pipelines without this sequencing anomaly, it was removed from the analysis.

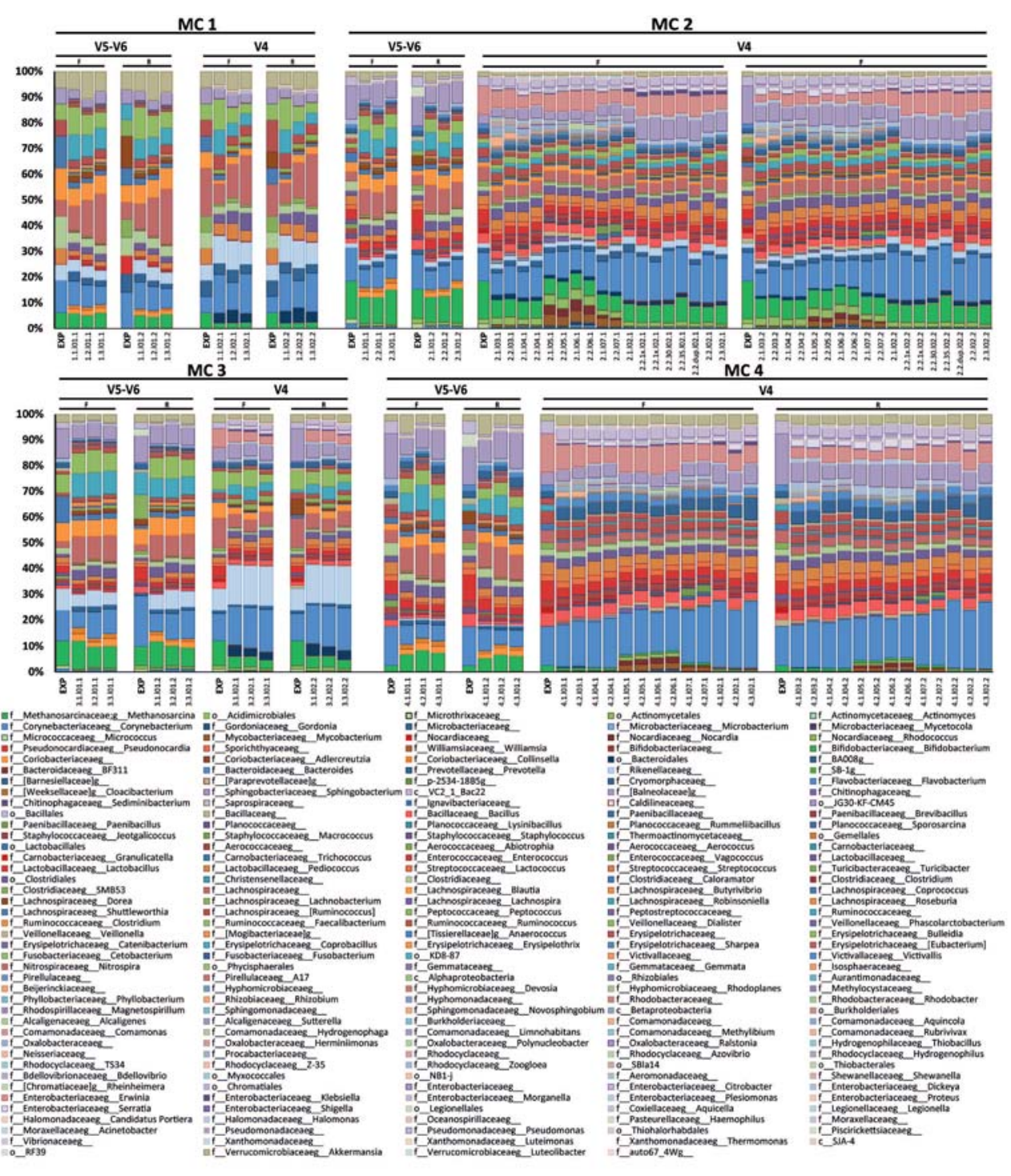

Figure 3. Observed composition of all MCs compared with the expected ones (EXP) for both regions and each read separately obtained with QIIME. 


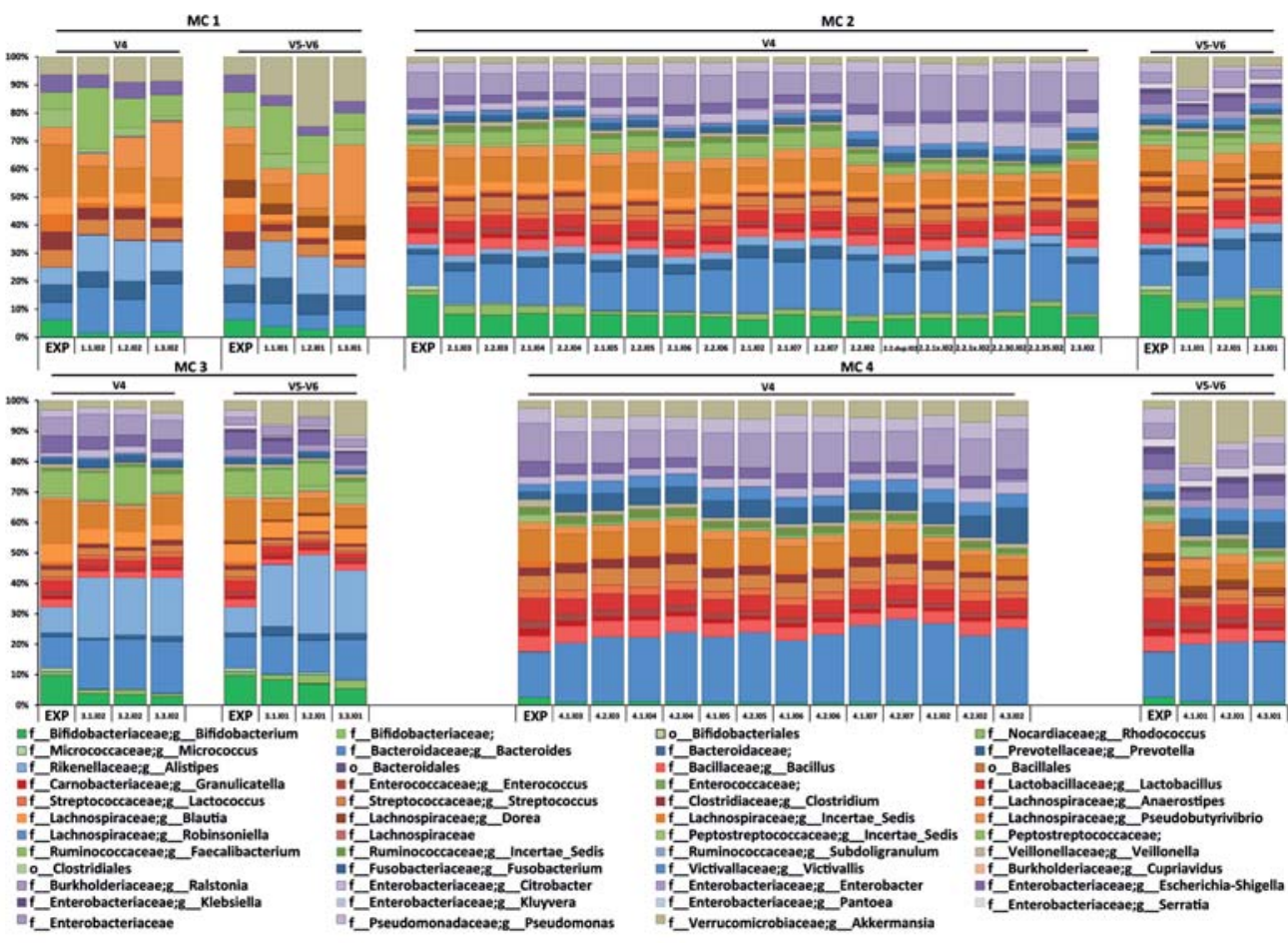

Figure 4. Observed composition of all MCs compared with the expected ones (EXP) for both regions obtained with NG-tax.

\section{Observed versus expected diversity}

Richness and diversity measures are important for understanding community complexity and dynamics. Among these measures, $\alpha$-diversity is defined as the diversity within a sample, which is often estimated based on the abundance distribution (evenness) and number (richness) of species, whereas $\beta$-diversity is defined as the partitioning of diversity among communities. The ability of researchers to quantify richness and diversity hinges on an accurate assessment of the composition of these communities ${ }^{353}$. For microbial communities, this has been particularly challenging, as none of the existing molecular microbial ecology methods normally capture more than a small proportion of the estimated total richness in most microbial communities ${ }^{354}$. For deep sequencing based approaches, filtering strategies that remove low-abundance reads make it impossible to apply richness estimation metrics such as the Chaol index and the ACE coverage estimator, because lowabundance read counts are included in their calculations. Conversely, richness estimates based on unfiltered datasets are unlikely to be accurate, if many of the reads actually represent PCR and/or sequencing errors ${ }^{333}$. In contrast to purely OTU-based methods, divergence-based methods account for the fact that not all species within a sample are equally related to each other, considering two communities to be similar if they harbour the 
same phylogenetic lineages, even if the species representing those lineages in each of the communities are different. Consequently, these methods are more powerful than purely OTU-based methods, because similarity in $16 \mathrm{~S}$ rRNA gene sequence often correlates with phenotypic similarity in key features such as metabolic capabilities. An added benefit is that small errors that are likely due to unfiltered sequencing errors, are punished less severely because OTUs that are only a few nt distant from each other due to error are still closely related using divergence based indices. Therefore, these indices probably provide a better estimate of the true diversity for data generated by high throughput next generation technology sequencers.

Because the focus of NG-Tax was to retain as much biological signal as possible while minimizing the impact of any technical choice, divergence-based $\alpha$-diversity (Phylogenetic Diversity (PD) ${ }^{355}$ ) and $\beta$-diversity (Unifrac ${ }^{353}$ ) metrics were used to visualize the diversity within and between MCs (Figure 5). The results obtained with QIIME suffered from all of the previously described technological artifacts. The MCs clustered by primer pair instead of MC, and within each cluster the structure, i.e. the position of MCs relative to each other, was different. More importantly, the true biological variation depicted by the expected composition was reproduced by neither primer pair (Figure 5C). Based on these results not only the Principle Coordinates Analysis ( $\mathrm{PCoA}$ ) based conclusions would have been different for both primer pairs, but also the differences in taxonomic classification could lead to significant changes in identified biomarkers, in line with what has previously been observed by $\mathrm{He}$ and co-workers ${ }^{342}$. Here we show that replicability within a variable region was attained. The more important reproducibility, however, i.e. the corroboration of findings by reproduction in different independent setups that use e.g. different primers, was not. This is an important observation because biological findings should be insensitive to independent methodologies ${ }^{330}$. In line with the above, also the observed $\alpha$-diversity (PD) was found highly inflated and the biological order was not reproduced (Figure 5D). In contrast, NG-Tax provided a clear separation of samples by MC type and their representative expected samples regardless of variable region, PCR protocol, sequencing run, library and sequencing depth. These results are remarkable, given the biases associated with each of these categories and the difference in resolution between the two regions (Figure 5A). Moreover, MC2, MC3 and MC4 were very similar, sharing the same genera and largely the same phylotypes, only differing in relative distribution (Table 1). Correspondingly, rarefaction curves for $\alpha$-diversity (Figure 5B) showed excellent reproduction of the true diversity. A perfect overlap cannot be achieved since the expected MCs do not account for sequencing or PCR errors, and these factors cannot be completely removed from real sequencing data. 


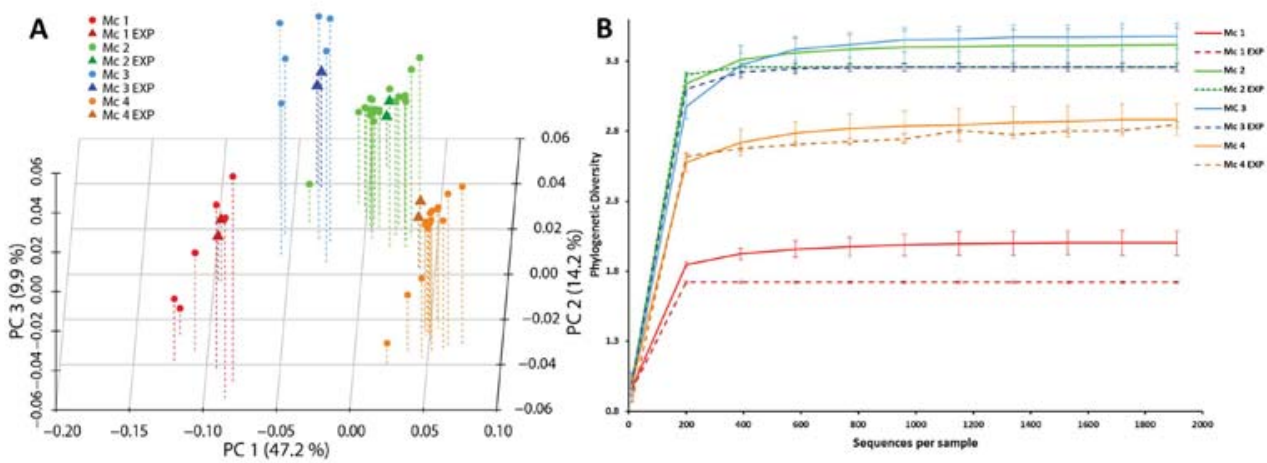

C

4

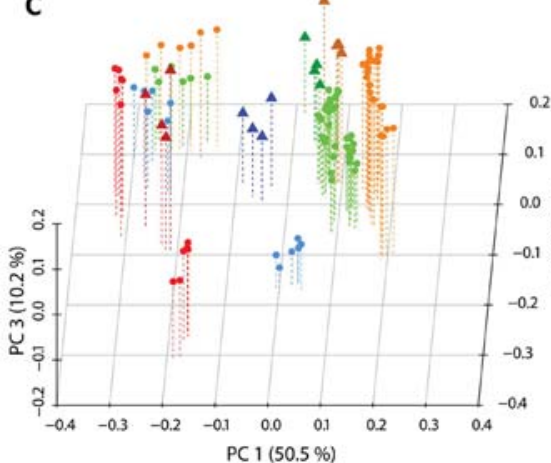

D

Figure 5. PCoA using Weighted Unifrac of all sequenced and expected MCs from NG-Tax (A) and QIIME (C), darker colored triangles represent the expected composition while lighter colored circles represent sequenced samples. Rarefaction curves of PD for all MCs and their expected counterparts from NG-Tax (B) and QIIME (C). These plots illustrate the excellent representation of the true diversity by the sequenced data using NG-Tax.

Small distances to expected MCs show the accuracy of NG-Tax, reproducibility on the other hand can be evaluated by the within MC distances and the dispersion of the between MCs distances (Figure 6). Distances to the expected MCs, within MC distances and dispersion of the between MCs distances were significantly lower $(\mathrm{p}<1 \mathrm{e}-10)$ for NG-Tax compared to QIIME. K-means cluster prediction using within groups sum of squares predicted 2 clusters for QIIME (Supplementary Figure 2) and the correct 4 clusters for NGTax (Supplementary figure 3). 


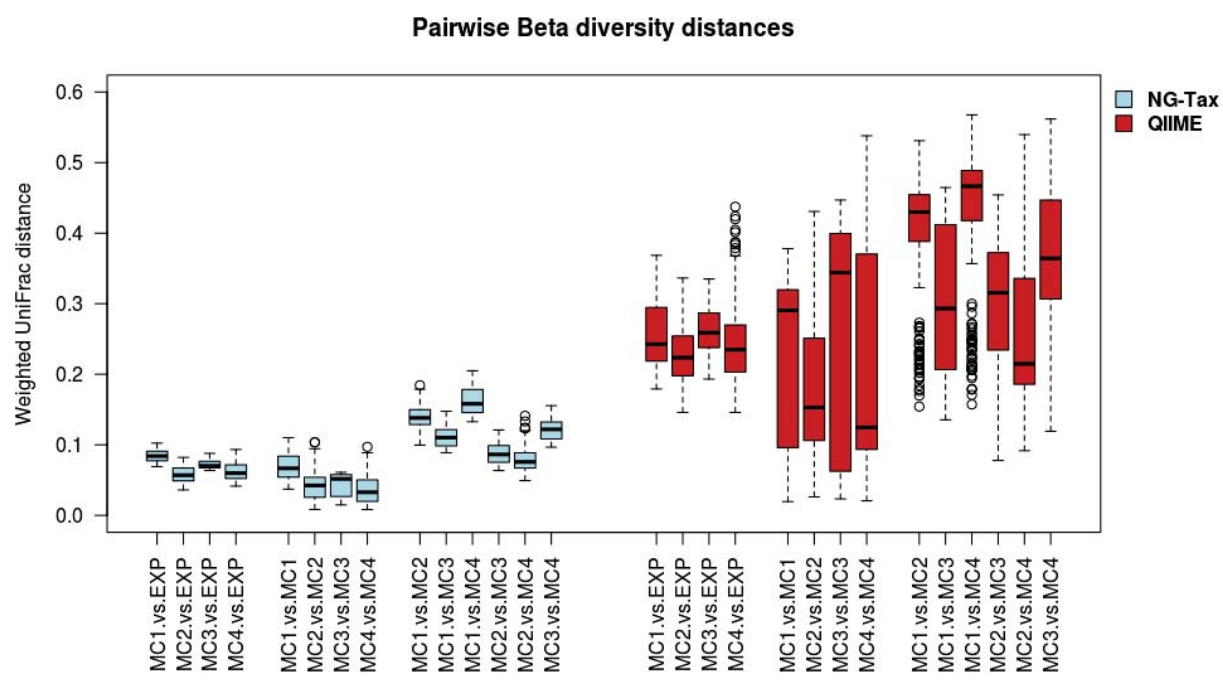

Figure 6. Pairwise Weighted UniFrac distances. Distances to the expected MCs, within MC distances and dispersion of the between MCs distances were significantly lower $(\mathrm{p}<1 \mathrm{e}-10)$ for NG-Tax compared to QIIME. NG-Tax results are depicted in blue and QIIME in red.

\section{Conclusions}

An increasing number of studies have shown that the chosen methodology rather than the

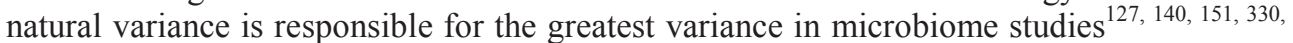
347-349. Some authors raised their concern with comparing data generated using different strategies $^{343}$, which basically suggests that true reproducibility (i.e. using different approaches and drawing the same biological conclusions) cannot be attained. This is an alarming observation since studies are often used to identity biomarker organisms, associated with certain host phenotypes (often comparing a diseased state to a healthy state), yet the use of different primers might show different biomarkers ${ }^{127,128,151,340,342,345}$. So far, neither currently available pipelines nor taxonomic classifiers have been able to efficiently reduce the noise in this type of data. Nevertheless, in properly de-noised datasets, taxonomical profiles, richness and diversity should be close to the expected values and the abundance of unassigned and poorly assigned reads should be low except when dealing with largely unexplored environments that are not sufficiently covered yet by the reference databases. At lower noise levels different variable regions should yield similar conclusions with small variations due to region specific resolution, and minor changes in the experiment should still deliver the same biological conclusions. Here we presented NGTax, an improved pipeline for 16S rRNA gene amplicon sequencing data, which continues to be a backbone in the analysis of microbial ecosystems. Several novel steps ensure much 
needed improved robustness against errors associated with technical aspects of these studies, such as PCR protocols, choice of 16S rRNA gene variable region and variable rates of sequencing error ${ }^{127,343,347}$. The commonly reported problems such as many un- or poorly classified OTUs, inflated richness and diversity, taxonomic profiles that do not match the expected ones, region dependent taxonomic classification and results being highly dependent on minor changes in the experimental setup have been tackled with NG-Tax. Despite the short read length $(\sim 140 \mathrm{nt})$ and all technical biases, the average taxonomic assignment specificity for the phylotypes included in the MCs was $96 \%$. In addition, $92.02 \%$ of the reads could be assigned to at genus level and $99.94 \%$ to at least family. Spurious genera represented only $0.02 \%$ of the reads per sample. More importantly, rarefaction curves and PCoA plots confirmed improved performance of NG-Tax with respect to clustering based on biology rather than technical aspects, such as sequencing run, library or choice of $16 \mathrm{~S}$ rRNA gene region. Therefore NG-Tax represents a method for $16 \mathrm{~S}$ rRNA gene amplicon analysis with improved qualitative and quantitative representation of the true sample composition. Additionally, the high robustness against technical bias associated with 16S rRNA gene amplicon studies will improve comparability between studies and facilitate efforts towards standardization.

\section{Methods}

\section{Primers}

Primer pairs 515F (5'-GTGCCAGCMGCCGCGGTAA) - 806R (5'GGACTACHVGGGTWTCTAAT) and BSF784F (5'-RGGATTAGATACCC) - 1064R (5'-CGACRRCCATGCANCACCT) have been previously reported for amplification of the $\mathrm{V} 4{ }^{128}$ and $\mathrm{V} 5-\mathrm{V}^{127}$ regions of the bacterial $16 \mathrm{~S}$ rRNA gene, respectively. They were selected based on 1) experimental validation, 2) taxonomic coverage of the relevant ecosystem (Supplementary Figure 4) adherence to specific rules associated with the sequencing platform, such as a maximum amplicon size of $<500 \mathrm{nt}$. Unless noted otherwise all primers were ordered at Biolegio (Nijmegen, Netherlands).

\section{Barcoding strategy}

At the time of sequencing Illumina's Hiseq2000 allowed for multiplexing of up to 12 samples per lane using an index or barcode read provided by Illumina. To achieve optimal sample throughput and phylogenetic depth, 70 primers containing a custom designed 8nt barcode were developed to combine with the Illumina barcodes to reach a maximum throughput of $12 \times 70$ samples per lane. Each set of 70 barcoded samples are referred to as "library". Low diversity samples, such as 16S rRNA gene amplicons, can lead to problems with base calling due to overexposure of fluorescent labels. Therefore, the set of 70 barcodes was specifically designed to possess an equal base distribution over their complete 
length. Additionally, to avoid differential amplification, a two-base "linker" sequence that is not complementary to any $16 \mathrm{~S}$ rRNA sequence at the corresponding position, from a database that contains 1132 phylotypes associated with the Human GI tract ${ }^{159}$, was inserted between the primer and barcode. The resulting set of 70 barcoded primers was checked for avoidance of secondary structure formation within or between primers (i.e., primer-dimers) or between barcodes and primers, using PrimerProspector ${ }^{148}$.

\section{Mock communities}

All MCs were mixed in triplicate to account for pipetting error. These MCs ranged from 17-55 species in both equimolar and staggered compositions. One MC contained members at very low abundances of $0.1,0.01$ and $0.001 \%$ (Table 2). Amplicons were generated either from cloned 16S rRNA gene amplicons, isolates available in the local culture collection of the Laboratory of Microbiology, Wageningen University, or strains ordered from DSMZ and cultured according to DSMZ recommendations, after which genomic DNA was isolated using the Genejet genomic DNA isolation kit (Thermo fisher scientific AG, Reinach, Zwitserland). A 16S rRNA gene specific PCR was performed using the universal primers 27F (5'-GTTTGATCCTGGCTCAG) - 1492R (5'GGTTACCTTGTTACGACTT) to obtain full length amplicons of which size and concentration were checked on a $1 \%$ agarose gel and which were column purified and quantified with the Qubit 2.0 fluorometer, and dsDNA BR assay kit (Invitrogen, Eugene, USA). Amplicons were mixed in the MCs to obtain the specified relative abundances. High quality full length reference sequences of all MC members were obtained by Sanger sequencing at GATC Biotech AG (Constance, Germany) with sequencing primers 27F $1492 \mathrm{R}$ in order to confirm their identity. The MCs were diluted $10^{3}$-fold and subsequently used as templates in PCRs for the generation of barcoded PCR products.

\section{Barcoded PCR}

Unless noted otherwise, each sample was amplified in triplicate using Phusion hot start II high fidelity polymerase (Thermo fisher scientific AG), checked for correct size and concentration on a $1 \%$ agarose gel and subsequently combined and column-purified with the High pure PCR cleanup micro kit (Roche diagnostics, Mannheim, Germany). Forty $\mu$ l PCR reactions contained $28.4 \mu \mathrm{L}$ nucleotide free water (Promega, Madison, USA), $0.4 \mu \mathrm{L}$ of $2 \mathrm{U} / \mu \mathrm{l}$ polymerase, $8 \mu \mathrm{L}$ of $5 \times \mathrm{HF}$ buffer, $0.8 \mu \mathrm{l}$ of $10 \mu \mathrm{M}$ stock solutions of each of the forward (515F) and reverse (806R) primers, $0.8 \mu \mathrm{L} \mathrm{10mM} \mathrm{dNTPs} \mathrm{(Promega)} \mathrm{and} 0.8 \mu \mathrm{L}$ template DNA $\left(10^{3} \times\right.$ diluted $200 \mathrm{ng} / \mu \mathrm{l}$ stock $)$. Reactions were held at $98{ }^{\circ} \mathrm{C}$ for $30 \mathrm{~s}$ and amplification proceeding for 25 cycles at $98{ }^{\circ} \mathrm{C}$ for $10 \mathrm{~s}, 50^{\circ} \mathrm{C}$ for $10 \mathrm{~s}, 72^{\circ} \mathrm{C}$ for $10 \mathrm{~s}$ and a final extension of $7 \mathrm{~min}$ at $72{ }^{\circ} \mathrm{C}$. Purified amplicons were quantified using Qubit. For primer pair BSF784F-1064R the thermal cycling conditions were identical to those detailed above except that the annealing temperature was $42^{\circ} \mathrm{C}$. To quantify noise generated by the PCR protocol, several reactions were performed with 30 or 35 cycles and $1 \times 100 \mu 1$ reaction 
instead of pooling $40 \mu \mathrm{l}$ in triplicate (Table 2). A composite sample for sequencing was created by combining equimolar amounts of amplicons from the individual samples, followed by gel purification and ethanol precipitation to remove any remaining contaminants. The resulting libraries were sent to GATC Biotech AG for sequencing on an Illumina Hiseq2000 instrument.

\section{Sequence analysis with QIIME}

We have used QIIME to benchmark NG-Tax. Illumina fastq files were de-multiplexed, quality filtered and analyzed using QIIME (v. 1.9) ${ }^{350}$ with closed reference OTU picking, using default settings and quality parameters as previously reported ${ }^{333}$. An additional analysis using a $0.1 \%$ abundance threshold can be found in Dataset 1 .

\section{NG-tax pipeline and user manual}

The NG-tax pipeline, user manual and files and code to reproduce the presented results, are available for download at http://www.systemsbiology.nl/NG-Tax/.

\section{Abbreviations}

rRNA: ribosomal RNA; MC: Mock Community; OTU: Operational Taxonomic Unit; PT: Phylotype; RDP: Ribosomal Database Project; RDPc: RDP classifier; PD: Phylogenetic Diversity; PCoA: Principle Coordinates Analysis

\section{Description of additional data files}

Dataset 1. Raw data of NG-Tax pipeline for analysis of 16S rRNA amplicons from complex biomes is available through the digital version of the manuscript at: http://f1000research.com/articles/5-1791/v1\#DS0 


\section{Supplementary figures}

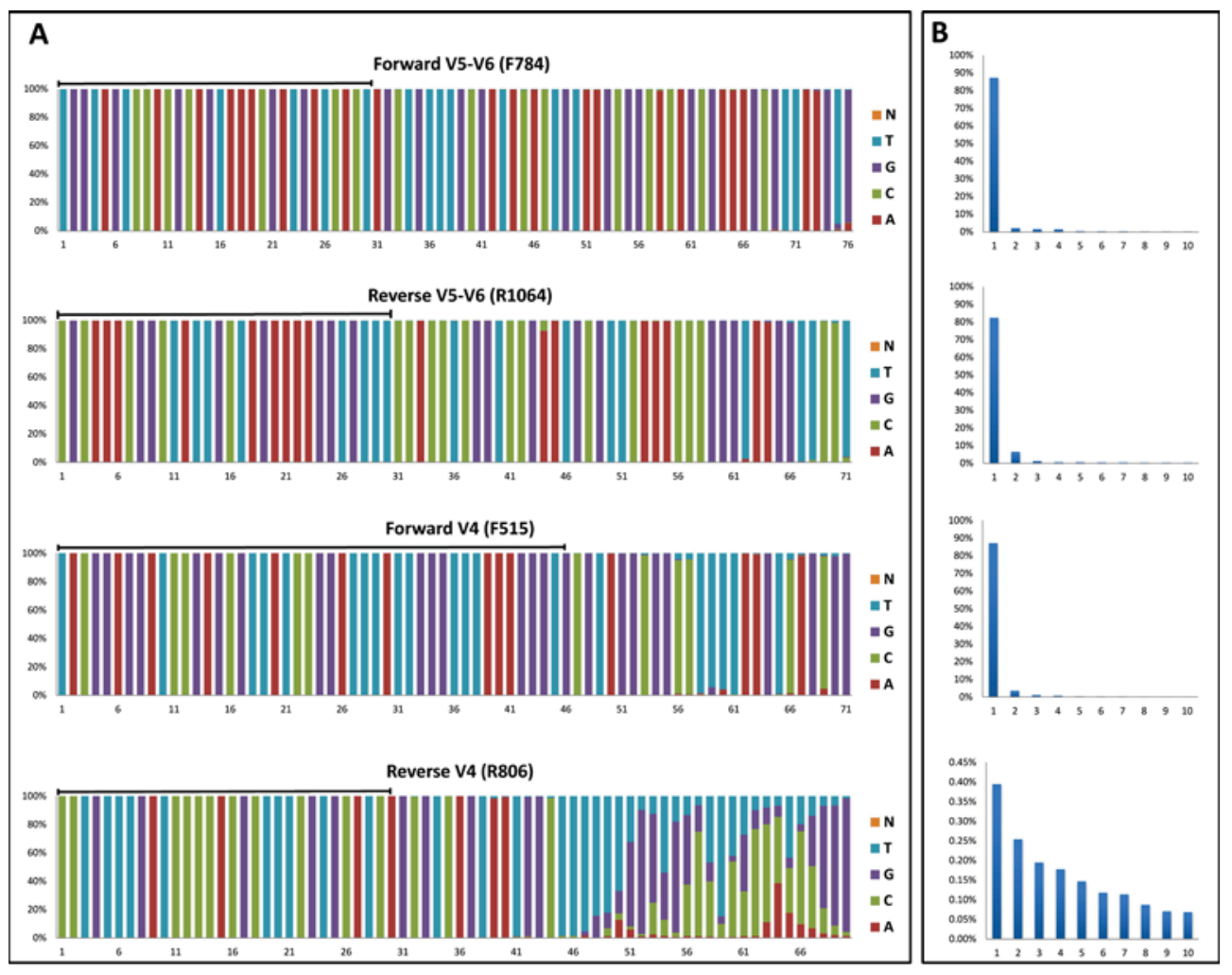

Supplementary figure 1. A) Nucleotide distribution of PT17 (Parabacteroides) for each of the four primers. Positions under the black segment are fixed and specific for PT17 preventing the inclusion of sequences belonging to a different PT. B) Percentage of 10 most abundant sequences for PT17 obtained with each of the primers.

PT17 (Parabacteroides) presented a sequencing anomaly in the reverse V4 region (primer R806) (Supplementary figure $1 \mathrm{~A}$ ). From positions 50 to 67 this region had higher error rate than the other three regions. The noise generated from this anomaly masked the biological signal rendering PT17 undetectable. In fact the most abundant sequence represented less than $0.45 \%$ of the total reads, while for the other three regions the most abundant sequence represented more than $80 \%$ (Supplementary figure 1B). We decided to remove the sequences belonging to PT17 from V5-V6 samples to avoid region clustering due to the presence of PT17. Our intention in this study was to test region performance under conditions in which sequencing anomalies like the one showed in Supplementary figure 1 are not present. 

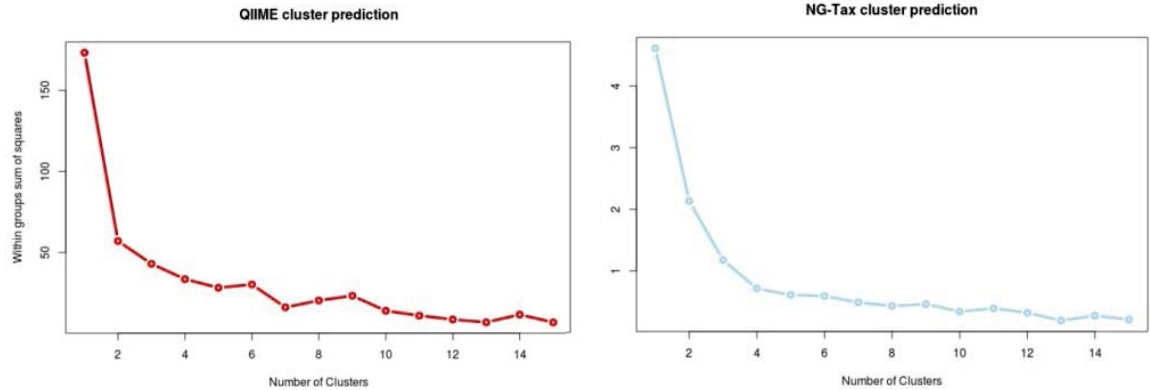

Supplementary Figure 2 \& 3. K-means cluster prediction for QIIME results and NG-Tax. The number of clusters was selected using the "elbow criterion". This looks at the percentage of variance explained as a function of the number of clusters. When the marginal gain of the explained variance drops it gives the line an angle, thus indicating the number of clusters.

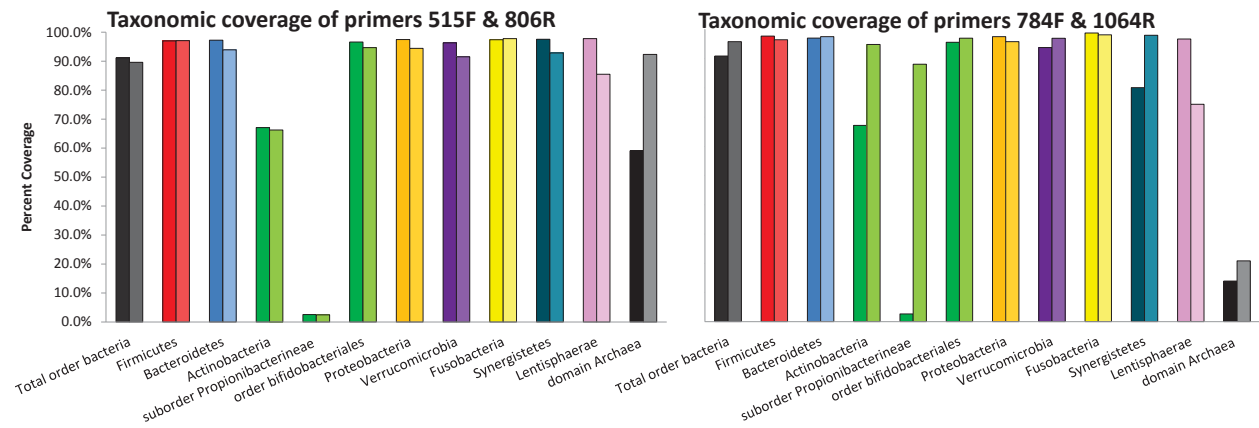

Supplementary Figure 4. Taxonomic coverage of primers. Forward (left bars) and reverse (right bars) primer coverage of the major bacterial phyla associated with the human GI tract using RDP's probematch program with one mismatch allowed. 


\section{Chapter 7}

\section{Dynamics of microbial community composition and activity in an in vitro gut model fed different diets}

Gerben D.A. Hermes*, Marisol Aguirre*,Javier Ramiro-García*, Dina Ripken,

Dominic Kaiser, Hauke Smidt, Erwin G. Zoetendal, Marjorie E. Koenen, Koen Venema

* These authors have contributed equally to this work 


\begin{abstract}
The aim of this study was to characterize the dynamics of microbial community composition and activity in an in vitro gut model (TIM-2) fed with either a normal diet, or modified versions from which the carbohydrate (MPLC) or protein fraction was diluted (LPMC) for $72 \mathrm{~h}$. We also assesed the robustness and reproducibility of the microbial responses. The production of short chain fatty acids and branched chain fatty acids reflected the quantity of carbohydrate and protein in the system. We monitored the developing microbial communities through $16 \mathrm{~S}$ ribosomal RNA gene sequencing of two variable regions, and overall, each diet produced distinct microbial communities and temporal patterns. The microbiota in reactors fed diets containing normal carbohydrate levels were enriched in members of the genera Prevotella, Subdoligranulum, Blautia and Bifidobacterium, all associated with carbohydrate fermentation. In turn, the microbiota in the reactors fed the MPLC diet, containing tenfold less carbohydrates, was enriched in the genus Bacteroides, which is associated with diets rich in protein and animal fat. These observations of distinct communities were corroborated at a global functional level by analysis of the metatranscriptome. The current setup will allow us to study the (trophic) interactions and task division within a community and how they are impacted by diet-related factors under controlled conditions. This may assist in defining causal links between specific microbial groups and their activities and diet-derived parameters.
\end{abstract}




\section{Introduction}

The human gut microbiota consists of a complex community with a higher genetic coding capacity than the host ${ }^{7}$. This densely inhabited ecosystem performs different essential functions for the host but has also been associated with a plethora of human pathologies. Therefore, many studies have investigated the potential to modulate microbial composition and metabolic activity with the aim of improving host health ${ }^{356}$. In vivo and in vitro models have served as valuable tools to investigate fermentation in the gastro-intestinal tract and to explore the complexity of microbe-microbe interactions ${ }^{56,57}$. In the particular case of in vitro models, many setups have been developed in order to study the impact of environmental variables. For instance, different applications have been used to assess the bioavailability of environmental contaminants $59,357,358$, the impact of fermentation of prebiotics ${ }^{359}, 360$, and mechanistic studies using stable isotopes have been performed to identify microorganisms involved in the degradation of specific dietary components ${ }^{361,362}$. In vitro gut fermentation models overcome many limitations of in vivo studies, which include issues of costs, human volunteer compliance, ethical approval, difficulties associated with sampling from different regions in the gut, and their inability to determine effects based solely on microbiota activity $^{56,57}$. However, different criteria need to be fulfilled by in vitro gut models before they can be considered valid for monitoring the effects of specific interventions/treatments on the microbiota. It is important to ensure repeatability, robustness and reproducibility of the model, and they must be capable to maintain the characteristic high diversity and functional complexity of the human gut microbiota $^{363}$.

The aim of this study was to compositionally and functionally characterize the microbial communities that develop in an in vitro model simulating the proximal colon: the TIM-2 system ${ }^{364}$ when fed with different diets for $72 \mathrm{~h}$. These diets were the standard simulated ileal efflux medium (SIEM) $)^{365}$, which simulates the average protein and non-digestible carbohydrate (CHO) consumed in a normal Western $\operatorname{diet}^{364}$. Variations thereof were made by diluting the protein or CHO fraction. These diets were termed Low Protein Moderate Carbohydrate (LPMC) and Moderate Protein Low Carbohydrate (MPLC), respectively. Simultaneously, we evaluated the ability of the TIM-2 system to reproduce the temporal response of the microbiota at the level of composition by $16 \mathrm{~S}$ ribosomal RNA (rRNA) gene amplicon sequencing, activity (meta-transcriptomics) and overall metabolic response by quantifying the short chain fatty acids (SCFA) acetate, propionate and butyrate, the branched chain fatty acids (BCFA) iso-butyrate and iso-valerate, and ammonia. Some of these metabolites, such as acetate, are intermediates in complex microbial foodwebs ${ }^{34}$. Therefore, in the context of this chapter the term production will signify the net effect of total microbial metabolism. 


\section{Materials and methods}

\section{TIM-2 in vitro model operation}

\section{Inoculum}

The inoculum used for the TIM-2 experiments consisted of an active, pooled fecal microbiota prepared from seven healthy volunteers (male: $n=3$, female: $n=4$, average age $=$ $42 \pm 13 \mathrm{y}, \mathrm{BMI}=24 \pm 1.9 \mathrm{~kg} / \mathrm{m} 2$ ). We have previously shown that pooling does not result in an aberrant microbiota composition or activity ${ }^{366}$. Fecal samples were collected using a container kit which was maintained under anoxic conditions by using anaerobic packs (AnaeroGenTM, Oxoid, Cambridge, UK). Samples were homogenized in an anaerobic cabinet $(80 \% \mathrm{~N} 2,10 \% \mathrm{CO} 2,10 \% \mathrm{H} 2)$ and used to inoculate a fed-batch fermentor simulating the human 'cecum' conditions as described earlier ${ }^{364}$. In short, $670 \mathrm{ml}$ food was inoculated with $80 \mathrm{~g}$ of pooled stools. The incubation was done at $37^{\circ} \mathrm{C}$ for $44 \mathrm{~h}$, and the fermentor was constantly flushed with gaseous nitrogen in order to guarantee anaerobic conditions. In addition, $1250 \mathrm{ml}$ of food was supplied in fed-batch mode during the incubation time. The resulting slurry was aliquoted, snap-frozen in liquid nitrogen and stored at $-80^{\circ} \mathrm{C}$ before inoculation in TIM-2.

\section{TIM-2 fermentation experiments}

The study was performed in two different experimental weeks in which six units were run in parallel each week. The distribution of the diets in each unit is shown in Table 1 . The TIM-2 system was flushed with $\mathrm{N}_{2}$ at $37^{\circ} \mathrm{C}$ for $96 \mathrm{~h}$ prior to introduction of the inoculum. The $\mathrm{pH}$ was kept at or above 5.8 by automatic titration with $\mathrm{NaOH}(2 \mathrm{M})$. A $30 \mathrm{ml}$ portion of fecal homogenate was used to inoculate the separate units for each experiment. Each unit was then filled to $120 \mathrm{ml}$ with $90 \mathrm{ml}$ of dialysate ${ }^{365}$. Immediately after inoculation, the microbiota was left to adapt to the new environment for $16 \mathrm{~h}$. During this period, the basal medium preparation (SIEM) was gradually introduced into the system in a total volume of $40 \mathrm{ml}$. After adaptation, the community was deprived from any medium for $2 \mathrm{~h}$ (starvation) in order to maximize the utilization of the tested diets by the microbiota ${ }^{367}$. After this starvation period, the test diets were added at $\mathrm{t} 0$ and at a rate of $2.5 \mathrm{ml} / \mathrm{h}$. A total of $180 \mathrm{ml}$ of the different diets was administrated over the $72 \mathrm{~h}$ of the test period.

Throughout the experiment, the luminal content was maintained at a level of approximately $120 \mathrm{ml}$ in each unit by a level sensor (liquiphant FTL20-0025, Endress+Hauser). In order to remove fermentation products and water from the lumen, a dialysis system consisting of a semi-permeable hollow membrane ran through the lumen ${ }^{368}$. For all experiments, the speed of the dialysis was set at $1.5 \mathrm{ml} / \mathrm{min}$.

At $\mathrm{t} 24$ and $\mathrm{t} 48,25 \mathrm{ml}$ of lumen sample was removed from the system to mimic the transit of material coming from the proximal and reaching the distal colon ${ }^{365}$. Additionally, a sample of the inoculum before adaptation was taken $(\mathrm{t}-16 \mathrm{~h})$. 
Luminal and dialysate sampling was performed at $0,24,48$ and $72 \mathrm{~h}$. Samples were stored at $-80^{\circ} \mathrm{C}$ until analysis.

Fermentation media

During the $16 \mathrm{~h}$ adaptation period, all TIM-2 units were fed with simulated ileal efflux medium (SIEM) as described by earlier ${ }^{365}$. After this adaptation period the units were fed with the normal, MPLC and LPMC diets.

The normal (control) diet consisted of the normal preparation of SIEM, containing TBCO (Tween 80, bacto-peptone, caseine and ox-bile) and the following carbohydrates: pectin, xylan, arabinogalactan, amylopectin, and starch. The LPMC diet (10:1 CHO: protein) consisted of SIEM that was modified by diluting the concentration of TBCO ten times while retaining the proportions of the rest of the ingredients. For the MPLC diet $(1: 10$ CHO: protein), the $\mathrm{CHO}$ fraction of the basal medium was diluted 10 times, while retaining the proportions of the rest of the ingredients.

\section{Microbiota profiling}

\section{DNA extraction}

For the extraction of genomic DNA, $250 \mu 1$ of luminal sample was mixed with $250 \mu \mathrm{l}$ of TE buffer (Tris-HCl pH 7.6, EDTA pH 8.0), $50 \mu 1$ 10\% (v/v) SDS (Ambion, Austin, TX, USA) and $500 \mu \mathrm{l}$ acid-phenol (Phenol:Water $(3.75: 1 \mathrm{v} / \mathrm{v}) ; \mathrm{pH}=4.45-5.68$; Invitrogen, Carlsbad, CA, USA). Samples were treated 3 times in a FastPrep (Precellys 24, Bertin Technologies, USA) at speed 5.5 for $45 \mathrm{~s}$ and later centrifuged at $13.400 \mathrm{~g}$ for $15 \mathrm{~min}$ at 4 ${ }^{\circ} \mathrm{C}$. DNA was subsequently purified by extraction with a Maxwell MDx (Promega, Madison, USA) following the "16 Tissue LEV Total RNA" protocol according to manufacturer's instruction.

Multiplex $16 \mathrm{~S}$ rRNA gene amplicon sequencing 16S rRNA gene amplicon sequencing was performed as described previously ${ }^{369}$. Shortly, Primer pairs 515F (5'-GTGCCAGCMGCCGCGGTAA) - 806R (5'GGACTACHVGGGTWTCTAAT) and BSF784F (5'-RGGATTAGATACCC) - 1064R (5'-CGACRRCCATGCANCACCT) were used to amplify the $\mathrm{V} 4{ }^{128}$ and $\mathrm{V} 5-\mathrm{V} 66^{127}$ variable regions of the bacterial 16S rRNA gene, respectively. A composite sample of all barcoded PCR reactions was created for sequencing by combining equimolar amounts of amplicons from the individual samples, followed by purification and concentration using magnetic beads to remove any remaining contaminants. The resulting libraries were sent to GATC Biotech AG (Konstanz, Germany) for sequencing on an Illumina Hiseq2000 instrument.

Microbiota composition profiling

Demultiplexing, OTU picking and taxonomic assignments using the Silva_111_SSU Ref database were done with NG-tax ${ }^{369}$ using default settings. Three datasets were created 
based on the V4, V5-V6 data and a combination of the two, by sequence prediction. In short, both datasets generate different OTUs and therefore cannot be directly compared, however, by predicting the missing regions (region V4 for V5-V6 and vice versa) based on the most repeated sequence in the reference database we could combine the information of the two datasets and improve robustness against amplification bias and primer coverage. Statistical analysis and visualizations were done with QIIME v 1.9 $9^{350}$ and $\mathrm{R}$ version 3.1.3. Statistical differences of the relative abundance of specific taxa between diets were evaluated using a Kruskal Wallis test with false discovery rate correction (FDR). An FDR $<0.05$ was considered significant.

\section{Functional microbiota profiling: meta-transcriptome}

$R N A$ extraction and removal of $r R N A$

Total RNA was extracted from the luminal samples following a Macaloid-based RNA isolation protocol ${ }^{370}$, which incorporated the use of a phase lock gel ${ }^{\mathrm{TM}}$ (GLPTM) in order to effectively isolate the nucleic acid-containing phase from the sample as previously described $^{371}$. The RNeasy mini kit (QIAGEN, CA, USA) was used for RNA purification and included the use of an on-column DNAse I (Roche, Germany) treatment ${ }^{370}$ to remove DNA from the samples. RNA quantity and quality were assessed using a NanoDrop 2000/2000C spectrophotometer (Thermo Scientific, USA), Experion RNA Stdsens analysis kit (Biorad Laboratories Inc., USA) and plotting 23S/16S rRNA ratios (Experion ${ }^{\mathrm{TM}}$ Software V. 3.0, Bio-Rad, USA).

Extracted RNA was enriched for non-ribosomal RNA using the Ribo-Zero ${ }^{\mathrm{TM}}$ rRNA removal kit (Epicentre, Madison, USA) according to the manufacturer's instructions. The rRNA-depleted sample was purified by precipitation with ethanol as follows. The volume of each RNA sample was adjusted to $180 \mu \mathrm{l}$ using RNase-Free water and subsequently mixed with $18 \mu \mathrm{l}$ of $3 \mathrm{M}$ sodium acetate, $2 \mu \mathrm{l}$ of glycogen $(10 \mathrm{mg} / \mathrm{ml})$ and 3 volumes of icecold $100 \%$ ethanol. Tubes were stored overnight at $-20{ }^{\circ} \mathrm{C}$ and were subsequently centrifuged at $>10.000 \mathrm{~g}$ at $4{ }^{\circ} \mathrm{C}$ for $30 \mathrm{~min}$. The supernatant was carefully removed and the pellet was washed twice with $600 \mu \mathrm{l}$ ice-cold $70 \%$ ethanol. The pellet was allowed to airdry at room temperature for $5 \mathrm{~min}$. Finally, the pellet was dissolved in $10 \mu \mathrm{l}$ of RNase-Free water. The quality of the treated RNA was assessed using a NanoDrop 2000/2000C spectrophotometer (Thermo Scientific, USA) and Experion RNA Stdsens analysis kit (Biorad Laboratories Inc., USA). The Ribo-Zero ${ }^{\mathrm{TM}}$ treated RNA was used for library preparation using ScriptSeq ${ }^{\mathrm{TM}}$ (Epicentre, Madison, USA) according to the manufacturer's instructions. For the purification steps we used the HighPrep PCR kit (MagBio Genomics, Inc., US/Canada) according to the manufacturer's instructions. The samples were sent for sequencing at GATC-Biotech (Konstanz, Germany). 
Metatranscriptome analysis

Despite the enrichment of mRNA, rRNA depletion is generally incomplete. Therefore, remaining the rRNA sequences were removed with SortMeRNA ${ }^{372}$ as described previously ${ }^{204}$. Assembly of the unfiltered reads into contigs, prediction of ORFs (Open Reading Frame) and annotation with KEGG functions was done with the pipeline described by Davids et $a l^{61}$. Differential expression analysis of KEGG functions was performed with the $\mathrm{R}$ package edge $\mathrm{R}^{373}$.

\section{Functional microbiota profiling: metabolites}

SCFA (acetate, propionate and butyrate) and BCFA (iso-butyrate and iso-valerate) were quantified as described previously ${ }^{368}$. In brief, samples were centrifuged $(\sim 12.000 \mathrm{~g}, 5$ $\mathrm{min})$, and to the clear supernatant a mixture of formic acid (20\%), methanol and 2-ethyl butyric acid (internal standard, $2 \mathrm{mg} / \mathrm{ml}$ in methanol) was added. A $3 \mu \mathrm{l}$ sample with a split ratio of 75.0 was injected on a GC-column (ZB-5HT inferno, ID $0.52 \mathrm{~mm}$, film thickness 0.10 um; Zebron; phenomenex, USA) in a Shimadzu GC-2014 gas chromatograph. Ammonia was quantified by Bio-aNAlytiX (Mook, The Netherlands) ${ }^{374}$.

\section{Statistical analysis of metabolite data}

The experiments were performed in series of four replicates per diet (Table 1). Results are presented as mean \pm SD. Statistical analysis was performed using the SAS statistical software package (SAS version 9: SAS Institute, Cary, NC). Proc Gplots were used to test outcome variables for normality of distribution. The different metabolites were $\log$ transformed and compared in a mixed model. No outliers were detected. The ANOVA model considered diet and time (t0, t24, t48, t72) as fixed factors. The factor replicate, which is the number of performed experiments, was included in the model as a random factor. The Tukey-Kramer post hoc test was used to correct for multiple comparisons. The concentration of all metabolites was artificially set to zero at t0. Data were considered significant at $p<0.05$. 
Table 1. Experimental setup

\begin{tabular}{|c|c|c|c|}
\hline Diet & Date & Unit & Run \\
\hline Norm & week 1 & $\mathrm{Ma}$ & 1 \\
\hline Norm & week 1 & $\mathrm{Ma}$ & 1 \\
\hline LPMC & week 1 & $\mathrm{Mb}$ & 1 \\
\hline LPMC & week 1 & $\mathrm{Mb}$ & 1 \\
\hline MPLC & week 1 & $\mathrm{Mc}$ & 1 \\
\hline MPLC & week 1 & $\mathrm{Mc}$ & 1 \\
\hline Norm & week 1 & $\mathrm{Oa}$ & 2 \\
\hline Norm & week 1 & $\mathrm{Oa}$ & 2 \\
\hline LPMC & week 1 & $\overline{\mathrm{Ob}}$ & 2 \\
\hline LPMC & week 1 & $\mathrm{Ob}$ & 2 \\
\hline MPLC & week 1 & $\mathrm{Oc}$ & 2 \\
\hline MPLC & week 1 & $\mathrm{Oc}$ & 2 \\
\hline Norm & week 2 & $\mathrm{Ma}$ & 3 \\
\hline Norm & week 2 & $\mathrm{Ma}$ & 3 \\
\hline LPMC & week 2 & $\mathrm{Mb}$ & 3 \\
\hline LPMC & week 2 & $\mathrm{Mb}$ & 3 \\
\hline MPLC & week 2 & $\mathrm{Mc}$ & 3 \\
\hline MPLC & week 2 & $\overline{\mathrm{Mc}}$ & 3 \\
\hline Norm & week 2 & $\overline{\mathrm{Aa}}$ & 4 \\
\hline Norm & week 2 & $\mathrm{Aa}$ & 4 \\
\hline LPMC & week 2 & Ac & 4 \\
\hline LPMC & week 2 & Ac & 4 \\
\hline MPLC & week 2 & $\overline{A d}$ & 4 \\
\hline MPLC & week 2 & $\overline{A d}$ & 4 \\
\hline
\end{tabular}

\section{Results}

\section{Microbiota profiling}

The average number of reads per sample was $166311 \pm 131574$. To ensure robust and reproducible compositional data based on sequencing of $16 \mathrm{~S}$ rRNA gene amplicons, we amplified and sequenced two variable regions. To determine the resolution at which we could discern biological differences, several samples were processed in duplicate (PCR and sequencing). Furthermore, sequencing was performed in two independent runs. Weighted Unifrac distances ${ }^{375}$ (WU, a measure of community similarity) between technical replicates were significantly smaller than between samples assigned to different biologically relevant categories (e.g. different diets, different time points during fermentor runs, etc.) and between random samples ( $\mathrm{p}<2.2 \mathrm{e}-16)$, indicating a small technical error (Figure 1). 


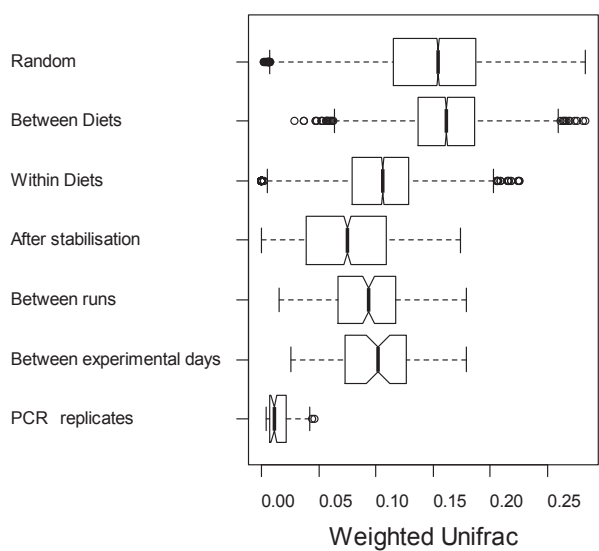

Figure 1. Boxplots showing weighted Unifrac distances for different categories. Within replicate distances are significantly smaller than other distances $(\mathrm{p}<2.2 \mathrm{e}-16)$ and within diet distances are significantly smaller than between diet distances $(\mathrm{p}<1 \mathrm{e}-10)$

\section{Community dynamics}

After inoculation and during the stabilization phase, i.e. comparing samples taken at $\mathrm{t}-16 \mathrm{~h}$ and t0, the diversity (Faiths phylogenetic diversity ${ }^{355}$ ) dropped significantly. Consequently, the composition at t0 was characterized by high relative abundances of mainly facultative anaerobes, such as Streptococcus, and Lactococcus (Figure 3), although not representative of the colon, this is a normal observation when using this system with this setup. Nevertheless, this drop in diversity and the resultant community was reproducible as no significant difference in diversity and relative abundance at genus level between units and replicates was found (FDR $>0.05)$. Therefore, all experiments started with similar initial communities, which were very different from the rest of the communities at other time points during the intervention. The largest shift in community composition occurred in the first $24 \mathrm{~h}$ for all diets $(p<0.0001)$ and the smallest change was in the MPLC diet $(p<0.0001)$. After $t 24$ the rate of change stays at the same level for the LPMC and normal diet, but for the MPLC diet the change slowed down, with a smaller distance between t24-48 than between $\mathrm{t} 48-\mathrm{t} 72(\mathrm{p}<0.0001)$ (Figure 2A). This is corroborated by a trend in diversity, which shows a similar pattern of increasing diversity throughout the experiment except for the MPLC diet, which shows a slight drop, indicative that an equilibrium has been reached (Figure 2B). After the addition of the different diets distinct communities formed (Figure 2C). To this end, unweighted unifrac (UU) based Principle Coordinate Analysis (PCoA) showed more pronounced separation of t0 samples from those taken during the fermentation in the presence of the different diets than the corresponding WU-PCoA, indicating that the developing communities were not only distinct based on differential abundance of taxa, but also regarding their presence. 

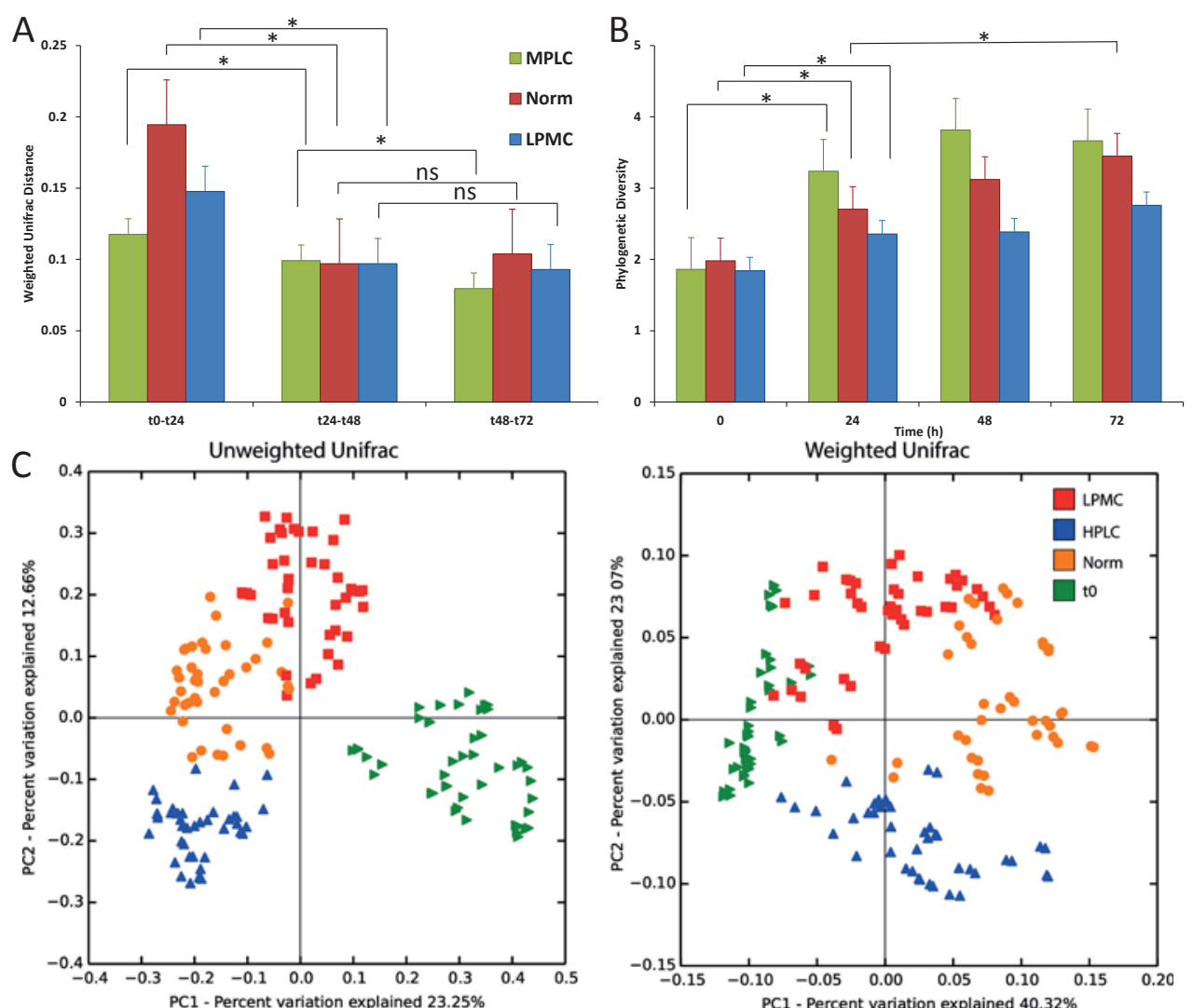

Figure 2. Community dynamics. (A) Weighted unifrac distances between consecutive timepoints and (B) phylogenetic diversity show a deceleration of community change for the MPLC diet but not for the normal and LPMC diet. (C) PCoA plots based on unweighted and weighted unifrac distances show formation of distinct communities after addition of the diets.

\section{Succession patterns}

After an initial large compositional shift from the Bacilli dominated composition at t0, all diets deviated towards a distinct composition (Figure 2C). The microbial communities developing in the TIM-2 model in the presence of the MPLC diet were most distinct regarding composition as well their temporal dynamics. Compared to the other diets, MLPC-fed fermenters showed signs of increased stability after 48h based on diversity, WU distances at consecutive time points and patterns of succession (Figures 2,3). Despite the minor differences in composition between the TIM-2 units and in different replicate runs (Figure 3), the diet effect was clearly visible (Figure 2C), and within diet WU distances were significantly smaller than between diet distances (Figure 1; $<<0.001$ ). Furthermore, the distances between experimental days and different runs were significantly smaller than between random samples, indicating a robust system. 
Although all diets resulted in distinct communities (Figure 2C), some patterns of succession were visible within all diets and units, except for one unit $(\mathrm{Ob})$, which showed a slightly deviant pattern compared to the other units with the same diet (Figure 3). Overall, members of Clostridium, Enterococcus and Streptococcus decreased while Prevotella increased.

Along with Prevotella, also Subdoligranulum, Blautia and Bifidobacterium increased in relative abundance in the normal and LPMC diets up to 448 . However, at 148 differences emerged between the normal and LPMC diets in the relative abundance of Dorea, Ruminococcus and to a lesser degree Lachnospiraceae;g_Incertae_Sedis, which increased in the normal diet but not in the LPMC diet.

Microbial communities that developed in the presence of the MPLC diet showed a different succession pattern compared to the other two diets. At $\mathrm{t}=24$, relative abundance of Prevotella, Bacteroides and Lachnospiraceae;g_Incertae_Sedis increased, while in contrast, the taxa that increased in the other the diets with ample CHO (Subdoligranulum, Blautia and Bifidobacterium) showed a slight decrease. At $48 \mathrm{~h}$ Prevotella decreased in relative abundance in the MPLC diet-fed units, while Bacteroides and Lachnospiraceae;g_Incertae_Sedis continued to increase in relative abundance. Simultaneously, a member of the Lachnospiraceae family increased as well as Dorea and Ruminococcus, resembling the pattern in the normal diet. At $72 \mathrm{~h}$ only slight changes could be observed in the composition, but the overall pattern remained similar to that of samples taken at $48 \mathrm{~h}$.

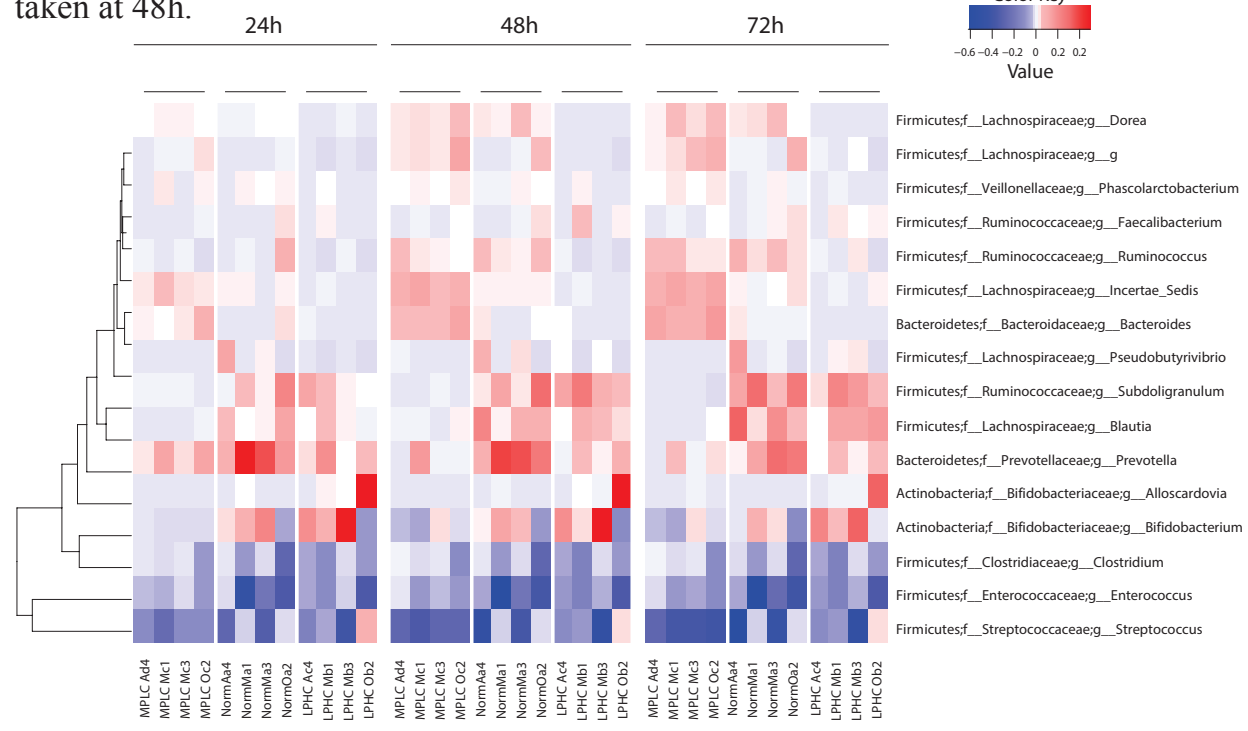

Figure 3. Succession patterns on each diet. Heatmap of genus level phylotypes whose relative abundance significantly different between the diets (FDR $<0.05$, Kruskal wallis test). Labels on the $\mathrm{x}$-axis show the diets in separate units. Colors depict change in relative abundance compared to t0 of the same unit. Blue depicts a decrease, red an increase. 


\section{Functional profiling: Metatranscriptome}

Unsupervised clustering of KEGG functionality revealed similar functionality at $\mathrm{t}=0$ and clustering by diet at $\mathrm{t}=24$. (Figure 4 ). At $\mathrm{t}=0$ none out of the 114573 identified functions were differentially expressed between the LPMC and the low CHO. After 24h the three diets displayed different functionality, with the MPLC diet being the most deviant. At this point 10040 (8.7\%) functions were differentially expressed between the MPLC and LPMC diets (data not shown).

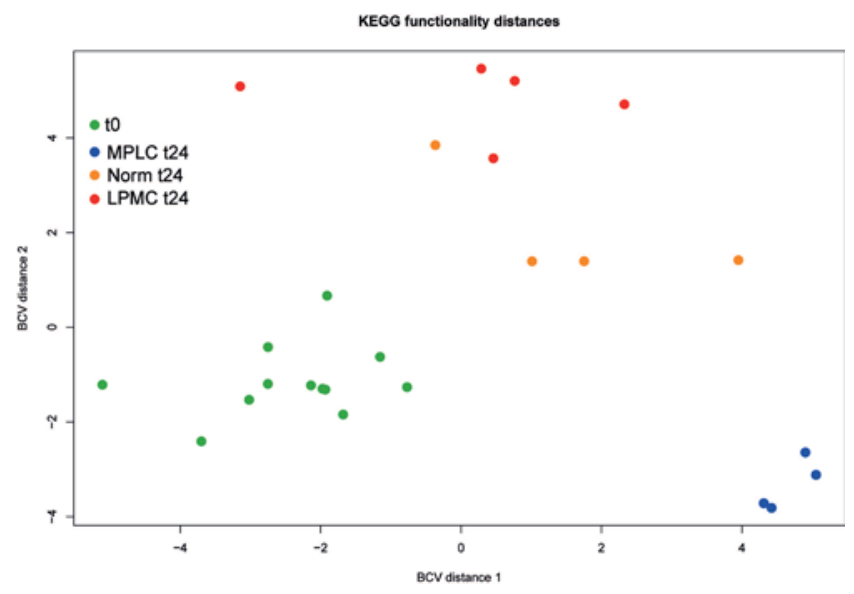

Figure 4. KEGG functionality distance plot of timepoint $\mathrm{t} 0$ and $\mathrm{t} 24$. Biological coefficient of variation $(\mathrm{BCV})$ is the distance based on biological abundance, independent of sequencing depth.

\section{Metabolites}

$p H$

The luminal $\mathrm{pH}$ in the TIM-2 system was maintained at 5.8. Therefore, $\mathrm{NaOH}$ consumption was used as a proxy of acid production in the lumen. The production of acid was significantly higher on the LPMC diet $(p<0.01$ at t72), and significant lower on the MPLC diet ( $p<0.01$ for t24, 48 and 72) compared to the normal diet ( Figure 5, Table S1). In addition, both LPMC and MPLC diets were significantly different from each other ( $p<$ 0.01 from t24 to t72). There was no statistically significant difference in $\mathrm{NaOH}$ consumed after the adaptation period between the three diets. 


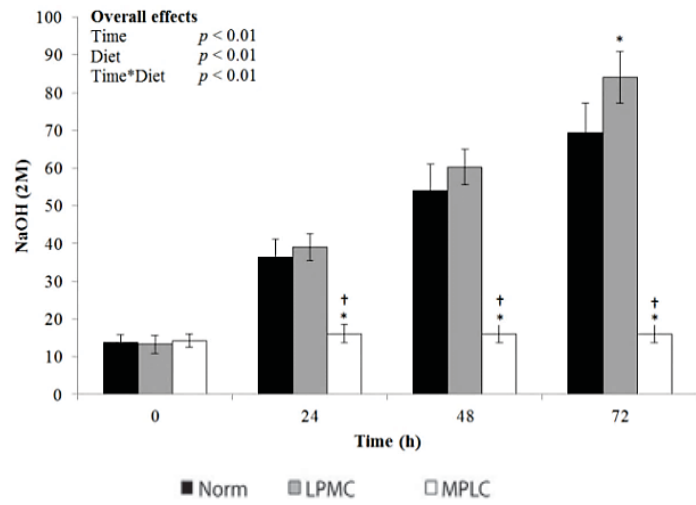

Figure 5. Cumulative consumption of $\mathrm{NaOH}(2 \mathrm{M})$ during the $72 \mathrm{~h}$ fermentations. Key: ${ }^{*} p<0.01$ specific time point is significantly different compared to the control diet; $\dagger p<0.01$ specific time point is significantly different compared to the LPMC diet.

\section{Total SCFA}

Both alterations of the normal diet resulted in lower total cumulative SCFA production (sum of acetate, propionate and $n$-butyrate) during the $72 \mathrm{~h}$ fermentation period (Table 2). The MPLC diet showed this trend from $\mathrm{t}=24$ onward, whereas the LPMC diet showed less SCFA production after $24 \mathrm{~h}$. After $72 \mathrm{~h}$ cumulative SCFA production was Normal $>$ LPMC $>$ MPLC, which is a reflection of the total calories from $\mathrm{CHO}$ in the respective diets.

Table 2. Total SCFA production. Total average (+/- SD) amount of SCFA (sum of acetate, propionate and n-butyrate; mmol) produced in the TIM- 2 system during the $72 \mathrm{~h}$ fermentation experiments with the three experimental diets. Values were artificially set to zero at the start of feeding the different test diets ( $\mathrm{t} 0)$.

\begin{tabular}{|l|c|c|c|c|}
\hline \multirow{2}{*}{ Diet } & \multicolumn{4}{|c|}{ Total SCFA (mmol) } \\
\cline { 2 - 5 } & $\mathrm{t} 0$ & $\mathrm{t} 24$ & $\mathrm{t} 48$ & $\mathrm{t} 72$ \\
\hline Norm & 0 & $41.8 \pm 3.12$ & $95.0 \pm 2.10$ & $150.4 \pm 4.09$ \\
\hline LPMC & 0 & $38.3 \pm 4.88$ & $72.3 \pm 8.37^{*}$ & $109.9 \pm 11.5^{*}$ \\
\hline MPLC & 0 & $22.9 \pm 2.29^{* \dagger}$ & $46.8 \pm 3.44^{* \dagger}$ & $72.3 \pm 3.85^{* \dagger}$ \\
\hline
\end{tabular}

Key: $* \mathrm{p}<0.01$ specific time point is significantly different compared to the normal diet; $\uparrow \mathrm{p}<0.01$ specific time point is significantly different compared to the LPMC diet.

\section{Acetate, propionate and n-butyrate}

A separate analysis of the production of acetate, propionate and $n$-butyrate revealed a similar pattern as the total SCFA. Compared to the normal diet, the cumulative production of each separate SCFA was significantly lower in the MPLC and LPMC diets at 72h, and at almost all time points (except for $n$-butyrate at $t=24$ ), the production of all SCFA was significantly lower in the MPLC diet. Between the two altered diets, only propionate showed no difference in cumulative production (Figure 6). 


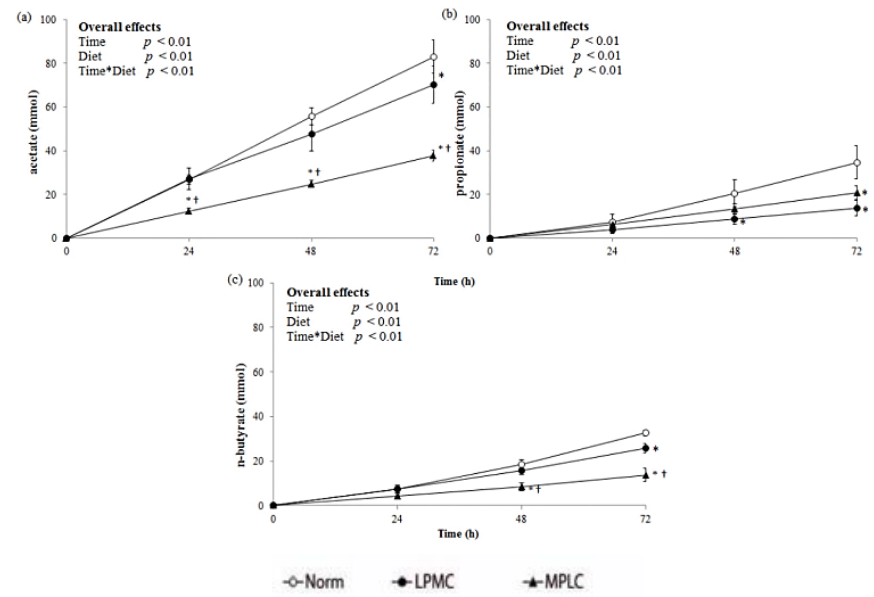

Figure 6. Kinetics of separate SCFA production. Average (+/- SD) kinetics of cumulative SCFA production (mmol) of (a) acetate, (b) propionate (c) and $n$-butyrate. Values were artificially set to zero at (t0). Key: ${ }^{*} p<0.01$ specific time point is significantly different compared to the normal diet; $\uparrow p<0.01$ specific time point is significantly different compared to the LPMC diet.

\section{$B C F A$ and ammonia}

After $72 \mathrm{~h}$ the MPLC diet showed the highest production of ammonia, total BCFA and both iso-butyrate and iso-valerate. In contrast the LPMC diet that contained ten times less protein showed almost no production of these metabolites. After $72 \mathrm{~h}$ cumulative BCFA, ammonia and iso-butyrate and iso-valerate production was Normal $>$ LPMC > MPLC, which is a reflection of the total calories from protein in the respective diets (Table 3, Figure 7,8).

Table 3. Total BCFA production. Average (+/- SD) total BCFA (sum of iso-butyrate and iso-valerate; mmol) produced in the TIM-2 system during the $72 \mathrm{~h}$ fermentation experiments with the three diets

\begin{tabular}{|l|c|c|c|c|}
\hline \multirow{2}{*}{ Diet } & \multicolumn{4}{|c|}{ Total BCFA (mmol ) } \\
\cline { 2 - 5 } & t0 & $\mathrm{t} 24$ & $\mathrm{t} 48$ & $\mathrm{t} 72$ \\
\hline Norm & 0 & $0.45 \pm 0.32$ & $1.32 \pm 0.54$ & $2.87 \pm 0.57$ \\
\hline LPMC & 0 & $0.00 \pm 0$ & $0.02 \pm 0.02^{*}$ & $0.23 \pm 0.29^{*}$ \\
\hline MPLC & 0 & $0.89 \pm 0.33^{\dagger}$ & $2.66 \pm 0.29^{* \dagger}$ & $5.33 \pm 0.36^{* \dagger}$ \\
\hline
\end{tabular}

Key: Values were artificially set to zero at the start (t0) $* p<0.01$ specific time point is significantly different compared to the normal diet; $\dagger p<0.01$ specific time point is significantly different compared to the LPMC diet. 


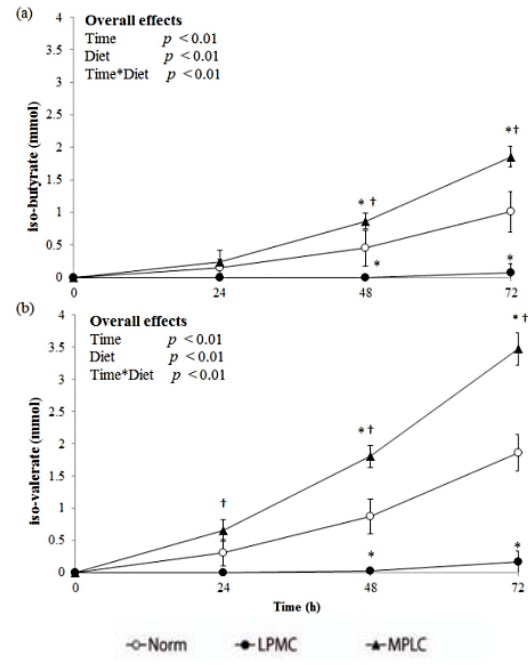

Figure 7. Production of separate BCFA.

Average (+/- SD) kinetics of cumulative BCFA production ( $\mathrm{mmol})$ in the experiments. (a) iso-butyrate, and (b) isovalerate. Values were artificially set to zero at ( $\mathrm{t} 0)$. Key: $* p<0.01$ specific time point is significantly different compared to the normal diet; $\uparrow p<0.01$ specific time point is significantly different compared to the LPMC diet.

Figure 8. Ammonia production. Average (+/- kinetics of cumulative ammonia production ( $\mathrm{mmol})$. Values were artificially set to zero at the (t0). Key: ${ }^{*} p<0.01$ specific time point is significantly different compared to the normal $\uparrow p<0.01$ specific time point is significantly different compared to the high $\mathrm{CHO}$ diet.

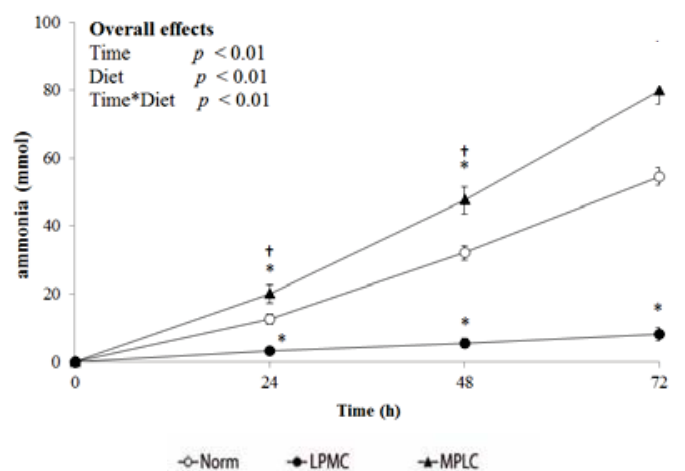

\section{Discussion}

Multiple versions of in vitro gut models are currently available, all of them presenting different levels of complexity in their design. Some in vitro models consist of a simple batch culture, some others are sophisticated continuous multistage (semi) continuous cultures $^{361,363,376}$. Both are characterized by the use of fecal microbiota as inoculum, and their operation is strictly controlled. The more sophisticated models are mostly performed at physiological temperature, $\mathrm{pH}$, food intake and anaerobic conditions ${ }^{363,377}$. Despite the efforts in closely simulating the human gut, in vitro gut models cannot be considered as an attempt to provide an exact replicate of the human intestine ${ }^{361,363}$. The complexity of host physiology is not fully reproduced in these models ${ }^{361}$. Rather, they are a potential tool to gain mechanistic insight in the process of fermentation and trophic interactions that occur in the human intestine. Therefore, efforts should be focused on their adequate conditioning in 
order to ensure their repeatability, robustness and reproducibility for experimental studies $^{363}$.

The TNO dynamic in vitro model of the proximal colon (TIM-2) is one example of a widely used model to study compositional and functional changes in the gut microbiota caused by different fermentable substrates. The operational conditions in TIM-2 are rigorously controlled and standardized. Furthermore, as described by Minekus et al., ${ }^{378}$, the model was validated comparing i) the composition, ii) the enzymatic activity and iii) production and concentration of SCFA from the microbiota with data from sudden death individuals. The system has been used for a wide range of analyses, including the study of metabolism of phenolic compounds ${ }^{379}$, the effect of prebiotics on the growth of bacteria ${ }^{364}$. ${ }^{380}$ and the use of different diets and intestinal microbiota ${ }^{381,382}$. We applied 16S rRNA gene amplicon sequencing and RNA-seq to characterize the development of microbial communities in TIM-2 fermenting a normal diet (SIEM) and two variations, including the LPMC diet from which the protein fraction was diluted 1:10 and the MPLC diet from which the $\mathrm{CHO}$ fraction was diluted 1:10, and studied the reproducibility and robustness of the system with four replicates per diet.

\section{Dietary impact on metabolite production}

In this study, we observed that the ratio of the dietary components were important determinants for the metabolic output and activity of the microbiota (Figure 5,6 and 7). The MPLC diet lead to an increased production of the toxic metabolites iso-valerate and iso-butyrate, which are known to result from the bacterial fermentation of valine and leucine ${ }^{383}$. In contrast, the normal and LPMC diets yielded a higher production of healthpromoting SCFA. The total cumulative SCFA production did not completely reflect the quantity of fermentable $\mathrm{CHO}$ in each of the diets. The MPLC diet yielded significantly lower quantities of SCFA (except for propionate) than the other diets. Although the total quantity of $\mathrm{CHO}$ was the same, the total yield of SCFA in the normal diet was significantly higher than the LPMC diet (Table 1). This could indicate less efficient substrate utilization due to the lower microbial diversity of the resultant communities (Figure 2B). However, the diets were not isocaloric so this might also be a reflection of less total substrate in the system. Nevertheless, we could corroborate the observation that moderate amounts of $\mathrm{CHO}$ could counteract some of the adverse metabolic consequences of high protein low carbohydrate diets ${ }^{384}$, as the production of BCFA remained significantly lower on the normal diet compared to the MPLC diet, even after $72 \mathrm{~h}$. Nevertheless, it is possible that carbohydrate fermentation was ongoing and therefore limited protein utilization as the gut microbiota preferably ferments $\mathrm{CHO}$ but switches to protein fermentation in case of $\mathrm{CHO}$ depletion $^{385,386}$. 


\section{Impact of the adaptation period on microbial communities}

The observed decrease in the diversity index found between the t-16 and t0 samples, indicates that the microbiota was affected during the adaptation period, which may be have been caused by parameters such as anaerobiosis, $\mathrm{pH}$, redox potential and substrate limitation, which may limit the optimal propagation of the inoculum in an in vitro system $^{387,388}$. This initial drop in microbial diversity and the subsequent recovery of the community has previously been observed ${ }^{389}$. Nevertheless, despite the compositional changes during the adaptation period, the composition of samples from all 12 experiments at t0 showed highly similar communities (Figure 2C), suggesting that the composition of the community was similarly affected in all experiments during the adaptation. The facultative anaerobes Streptococcus and Lactococcus were highly abundant after the adaptation period at t0. This has been previously observed in TIM2 and is possibly due to oxygen entering the system during inoculation ${ }^{390}$.

\section{Dietary impact on the development of microbial communities}

The microbial composition associated with the MPLC diet was the most distinct mainly due to the increased relative abundance of the genus Bacteroides and Lachnospiraceae; Incertae Sedis after $48 \mathrm{~h}$ and a lack of bifidobacteria during the fermentation (Figure 3). At 24h Prevotella were highly abundant but at the later time points it decreased in abundance concomitant with a simultaneous increase in relative abundance of Bacteroides. In literature the abundance of these genera has been associated with long term dietary habits. Prevotella has been associated with higher fibre consumption and that of Bacteroides with a higher intake of protein and fat ${ }^{391,392}$ probably mediated through the effect of bile acids that exert a strong selective influence upon gut microbial composition ${ }^{214}$. It should be noted that the normal diet contains just as much bile, yet the genus Bacteroides is absent. However, Prevotella might have been highly abundant due to the higher amount of $\mathrm{CHO}$ in this diet. It seems that the contrasting abundances of Prevotella and Bacteroides, which are the most distinct drivers for two of the microbiota types termed enterotypes ${ }^{92}$, was replicated in this experiment and seem to be linked therefore, presumably diets ${ }^{198}$.

The distinctive features of the LPMC diet compared to the MPLC diet were the lower diversity (Figure 2B) and abundance of Dorea, Lachnospiraceae; Incertae Sedis and Ruminococcus, which all appeared after $48 \mathrm{~h}$ in the other diets (Figure 3). Ruminococcus species as well as Roseburia are misclassified as Lachnospiraceae;_Incertae_Sedis due to ambiguity in their classification in 16S rRNA gene databases (results not shown, these were members of the synthetic communities described in chapter 6). All the members of these groups have been associated with the (primary) degradation of $\mathrm{CHO}^{362,393,394}$. It is possible that these taxa need either the protein, or the byproducts of protein synthesis to proliferate. This could explain the higher amounts of SCFA in the normal diet, as these species also produce SCFA. It is striking however, that these taxa do expand on a diet low in $\mathrm{CHO}$ and 
high in protein (MPLC) but not in a diet high in CHO that still contains some protein, albeit $10 \mathrm{x}$ less.

Microbial communities developing in the models fed with either of the two normal carbohydrate diets shared the growth of several groups that have been associated with carbohydrate metabolism in the human GI tract. Subdoligranulum has been reported to be highly abundant in vegetarians ${ }^{395}$ and enriched in pigs on a low protein $\operatorname{diet}^{396}$, demonstrating their adaptation to a low protein environment. Both genera Blautia ${ }^{397}$ and Bifidobacterium $^{362,398}$ have been associated with the fermentation of resistant starch, and the latter have been shown to primarily consume the intermediates produced by Ruminococcus related species during the primary degradation of $\operatorname{starch}^{362}$. Another striking observation is the mutual exclusion of high abundances of Bifidobacterium and Prevotella after $24 \mathrm{~h}$ of fermentation in some of the replicates of the LPMC diet. This could indicate that high abundances of Bifidobacterium take over the niche of Prevotella.

Most of the microbial development on the specific diets is in concordance with previous research. However, the high phylogenetic diversity observed in the MPLC is in contrast with the observation by Le Chatelier and colleagues who discerned a bimodal distribution of high gene count (HGC) and low gene count (LGC) individuals within the general population. The LGC group tended to be phylogenetically less diverse and dominated by Bacteroides, and these individuals also consumed less fibre ${ }^{196}$. Nevertheless, David et al. found no impact on diversity of a high protein high fat, low carbohydrate diet ${ }^{391}$. Although it seems we start to elucidate the effect of diet on the abundance of bacterial groups, and their impact on the production of beneficial as well as harmful metabolites, not all data is in full concordance indicating more research is needed in order to elucidate the impact different diets have on the microbiota and consequently gut health.

\section{Dietary impact on microbial activity}

Community composition based on 16S rRNA gene amplicons and microbial activity have been shown to be discordant ${ }^{399}$. Therefore, we determined microbial activity trough RNAseq at $\mathrm{t}=0$ and $\mathrm{t}=24$ and compared it with community composition. We observed a consistent robust and reproducible response in all diets (Figure 4). The fact that we did not find any differentially expressed functions at to confirmed the observation that the changes in microbiota after the adaptation period were similar and reproducible at the functional level. The vast majority of the expressed genes are household genes that are continuously expressed, thus revealing a high degree of similarity ${ }^{204}$. This was reflected by the fact that only $8.7 \%$ of the 114573 identified functions was significantly under- or overexpressed in the two altered diets (MPLC and LPMC). Unfortunately, $60 \%$ of all genes and all differentially expressed functions lacked a KEGG annotation, which subsequently hamper a detailed interpretation of the observations. 


\section{Conclusion}

The effects of different diets on microbiota composition and activity were repeatable, robust and reproducible in the different replicates through all experimentation weeks. Our findings not only showed the gut microbiota to be a highly metabolically adaptable organ ${ }^{180,391}$ but also highlighted the potential of the TIM-2 system as a tool for investigating the role of the microbiota on human health or disease through investigating reproducible changes in community structure and activity. This allows us to study the (trophic) interactions and task division within a community and how they are impacted by host- or diet-related factors under controlled conditions. This may assist in defining causal links between specific microbial groups and their activities, and host- or diet-derived parameters.

\section{Supplemental material}

Table S1. Fermentation metabolites produced during $72 \mathrm{~h}$ fermentation experiments. Key: ${ }^{*} p<0.01$ specific time point is significantly different compared to the normal diet; $\uparrow p<0.01$ specific time point is significantly different compared to the LPMC diet. The different diets correspond to: normal: 1:1 (control or basal SIEM medium); LPMC: 10:1 and HPLC 1:10 (CHO: protein) diets.

\begin{tabular}{|c|c|c|c|c|c|}
\hline \multicolumn{2}{|c|}{ Diet } & & & \multicolumn{2}{|c|}{ mmol } \\
\hline \multirow{10}{*}{ Norm } & & to & t24 & t48 & t72 \\
\hline & acetate & 0 & $26.8 \pm 2.10$ & $55.9 \pm 3.94$ & $83.1 \pm 7.54$ \\
\hline & propionate & 0 & $7.49 \pm 3.39$ & $20.5 \pm 6.16$ & $34.7 \pm 7.51$ \\
\hline & $n$-butyrate & 0 & $7.51 \pm 0.70$ & $18.7 \pm 1.91$ & $32.5 \pm 1.27$ \\
\hline & Total SCFA & 0 & $41.8 \pm 3.12$ & $95.0 \pm 2.10$ & $150.4 \pm 4.09$ \\
\hline & ammonia & 0 & $12.6 \pm 1.56$ & $32.1 \pm 2.08$ & $54.5 \pm 2.62$ \\
\hline & iso-butyrate & 0 & $0.15 \pm 0.13$ & $0.45 \pm 0.27$ & $1.01 \pm 0.31$ \\
\hline & iso-valerate & 0 & $0.31 \pm 0.20$ & $0.87 \pm 0.27$ & $1.86 \pm 0.28$ \\
\hline & Total BCFA & 0 & $0.45 \pm 0.32$ & $1.32 \pm 0.54$ & $2.87 \pm 0.57$ \\
\hline & Alkali & $13.88 \pm 1.91$ & $36.3 \pm 4.85$ & $53.9 \pm 7.13$ & $69.4 \pm 7.86$ \\
\hline \multirow[t]{9}{*}{ LPMC } & acetate & 0 & $27.2 \pm 4.89$ & $47.6 \pm 7.71$ & $70.4 \pm 8.54^{*}$ \\
\hline & propionate & 0 & $3.73 \pm 1.49$ & $8.77 \pm 2.56^{*}$ & $13.7 \pm 3.59^{*}$ \\
\hline & $n$-butyrate & 0 & $7.38 \pm 1.76$ & $15.8 \pm 1.97$ & $25.8 \pm 2.02^{*}$ \\
\hline & Total SCFA & 0 & $38.3 \pm 4.88$ & $72.3 \pm 8.37^{*}$ & $109.9 \pm 11.5^{*}$ \\
\hline & ammonia & 0 & $3.17 \pm 0.83^{*}$ & $5.48 \pm 1.38^{*}$ & $8.12 \pm 1.95^{*}$ \\
\hline & iso-butyrate & 0 & $0.00 \pm 0$ & $0.00 \pm 0^{*}$ & $0.07 \pm 0.14^{*}$ \\
\hline & iso-valerate & 0 & $0.00 \pm 0$ & $0.02 \pm 0.02^{*}$ & $0.16 \pm 0.17^{*}$ \\
\hline & Total BCFA & 0 & $0.00 \pm 0$ & $0.02 \pm 0.02^{*}$ & $0.23 \pm 0.29^{*}$ \\
\hline & Alkali & $13.33 \pm 2.38$ & $39.1 \pm 3.54$ & $60.2 \pm 4.66$ & $84.0 \pm 6.76^{*}$ \\
\hline \multirow[t]{9}{*}{ MPLC } & acetate & 0 & $12.4 \pm 1.35^{* \dagger}$ & $24.7 \pm 1.57^{* \dagger}$ & $37.8 \pm 2.47^{* \dagger}$ \\
\hline & propionate & 0 & $6.24 \pm 1.08$ & $13.4 \pm 2.52$ & $20.7 \pm 3.12^{*}$ \\
\hline & $n$-butyrate & 0 & $4.24 \pm 0.76$ & $8.50 \pm 1.82^{* \dagger}$ & $13.8 \pm 3.00^{* \dagger}$ \\
\hline & Total SCFA & 0 & $22.9 \pm 2.29^{* \dagger}$ & $46.8 \pm 3.44^{* \dagger}$ & $72.3 \pm 3.85^{* \dagger}$ \\
\hline & ammonia & 0 & $19.9 \pm 2.74^{* \dagger}$ & $47.5 \pm 4.05^{* \dagger}$ & $79.7 \pm 3.78^{* \dagger}$ \\
\hline & iso-butyrate & 0 & $0.24 \pm 0.17$ & $0.86 \pm 0.12^{* \dagger}$ & $1.86 \pm 0.16^{* \dagger}$ \\
\hline & iso-valerate & 0 & $0.65 \pm 0.17^{\dagger}$ & $1.80 \pm 0.18^{* \dagger}$ & $3.47 \pm 0.25^{* \dagger}$ \\
\hline & Total BCFA & 0 & $0.89 \pm 0.33^{\dagger}$ & $2.66 \pm 0.29^{* \dagger}$ & $5.33 \pm 0.36^{* \dagger}$ \\
\hline & Alkali & $14.28 \pm 1.80$ & $16.1 \pm 2.40^{* \dagger}$ & $16.1 \pm 2.40^{* \dagger}$ & $16.1 \pm 2.40^{* \dagger}$ \\
\hline
\end{tabular}





\section{Chapter 8}

General Discussion \& Summary 
Recent evidence indicates that the GI microbiota and its products may contribute to the development of obesity and related diseases, and that differences in GI tract microbial ecology between individuals might be an important factor in this complex etiology. This thesis aims to provide and apply tools to investigate the potential of the gut microbiota as a potential biomarker or therapeutic target and generate leads for mechanisms through which the microbiota mediates these effects.

Chapter 2 provides an overview of the different molecular approaches that are available to study the role of the microbiome in health and disease, highlights their respective strengths and creates awareness for the specific limitations of these complementary omics technologies such as 16S ribosomal RNA (rRNA) gene-targeted composition profiling, metagenomics, metatranscriptomics and metaproteomics. Although their specific application has contributed strongly to our understanding of the role of the GI microbiota in human energy homeostasis, it is currently still unclear which bacterial groups play a role in the development of obesity in humans, which might partly be explained by the heterogeneity in genotype, lifestyle and diet and the complex etiology of Obesity and its Associated Metabolic Disorders (OAMD). Nevertheless, recent research has shown a conceptual shift by focusing on more homogenous subpopulations, by using anthropometric (weight, total body fat) as well as biochemical variables (insulin resistance, hyperlipidaemia) for the stratification of subjects. Combined with technological advances in the molecular techniques, recent data suggests that an OAMD associated microbiota can be characterized by a potential pro-inflammatory composition (consisting of Proteobacteria), with lower potential for the production of SCFAs and butyrate in particular.

Chapter 3 describes the largest population-based study on childhood gut microbiota and body weight so far, the KOALA Birth Cohort, profiling the microbiota of 295 fecal samples from 6-7 year old children living in the south of the Netherlands. Using state of the art statistical methodology both new and previously described bacterial groups were identified to be either positively or negatively associated with weight related anthropometric outcomes (age- and sex- standardized weight and BMI Z-scores, overweight defined as $\mathrm{BMI} \geq 85$ th percentile). This age range is often overlooked in such type of studies. Therefore, we briefly investigated the structure of the microbiota with regards to well validated ecosystem properties such as the existence of bacterial tipping elements ${ }^{93}$. Although Lahti and co-workers investigated healthy adults we could confirm the bimodal distribution pattern of several bacterial groups as well as their co-correlating groups that were reported previously, including UCII, Prevotella spp. (P. oralis et rel. and $P$. melaninogenica et rel.), co-correlating with $P$. ruminicola et rel., and Dialister with uncultured Selenomodaceae. Lack of support for bimodality of UCI in our study is also in concordance with Lahti et al., who showed that UCI exhibits very clear shifting state probabilities associated with ageing, where the high abundance state was mainly observed above 40 years of age. Since the bootstrap support of bimodality for Bacteroides fragilis 
was only moderate in the study of Lahti et al., this group might have been missed in our study due to the smaller sample size, age, health status, or other biological factors.

Multivariate analysis revealed that weight-related outcomes failed to explain much of the observed variation in GI microbiota composition in the KOALA cohort. The abundance of the four bimodal groups explained $26.1 \%$ of the variation in species composition, compared to $<1 \%$ for each of the anthropometric outcomes. Therefore, we cannot confirm the often large-scale community shifts previously reported to be associated with obesity mainly in rodent studies ${ }^{48,164}$. These data suggests that in this group of healthy children within a relatively normal weight range, weight and associated parameters were not major drivers of overall microbial composition or vice versa. To reduce the dimensionality of the dataset to bacterial groups associated with weight related parameters and to avoid issues regarding correction for multiple testing we applied the Elastic net machine learning algorithm. The selected genus-like groups, bimodal taxa (linear as well as the high and low abundance states as a factor) and other features of the microbiota previously associated with (over)weight, such as the Bacteroidetes to Firmicutes (B:F) ratio, and richness and diversity ${ }^{36}$, were used in multiple linear and logistic regression models with adjustment for confounders to investigate their associations with weight related anthropometric outcomes in this cohort. The abundance of several specific bacterial taxa, including the dichotomous abundance state of the bimodally distributed UCII was consistently associated with weight-related outcomes. These include several bacteria that have previously been linked to weight-related outcomes (Akkermansia, UCII) ${ }^{65}$, as well as genus-like groups that have not previously been linked to (over)weight such as Sutterella wadsworthia et rel. and Bryantella formatexigens et rel. In this regard, more detailed information on their functional role in energy metabolism will help to establish their importance for weight development. Our results provide new avenues with regards to bacteria in the gut of humans in relation to the increasing trend of overweight worldwide. The parameters previously selected based on their merits from literature, i.e. diversity, richness and B:F ratio, were not significantly associated with any of the outcomes.

Chapter 4 investigated the effects of gut microbiota manipulation by antibiotics $(7 \mathrm{~d}$ administration of amoxicillin, vancomycin or a placebo) on tissue-specific insulin sensitivity, energy metabolism, gut permeability and inflammation in 57 obese, prediabetic men. Amoxicillin did not significantly affect microbial diversity and composition, whilst vancomycin decreased bacterial diversity and reduced Firmicutes. Among the most strongly affected groups were genus-like groups that contain known butyrate-producing species from Clostridium clusters IV and XIVa, such as Coprococcus eutactus, Faecalibacterium prausnitzii and Anaerostipes caccae, as well groups involved in BA dehydroxylation such as Clostridium leptum. These changes occurred concomitantly with altered plasma and fecal concentrations of these metabolites. In adipose tissue, gene expression of oxidative pathways was upregulated by antibiotics, whereas immune-related pathways were downregulated by vancomycin. However, antibiotic treatment had no significant effects on tissue-specific insulin sensitivity, energy/substrate metabolism, 
postprandial hormones and metabolites, systemic inflammation, gut permeability and adipocyte size. Importantly, despite a still considerably altered microbial composition at eight weeks follow-up, energy harvesting, adipocyte size and whole-body insulin sensitivity (HOMA-IR) remained unaltered. Overall these data indicate that interference with adult microbiota by antibiotic treatment for 7 days had no clinically relevant impact on metabolic health in obese humans.

These data are in contrast with several previous studies in rodents that indicated that antibiotic treatment may improve glucose homeostasis and metabolic impairments ${ }^{265-267}$, 293-295, 400 . Nevertheless, a more recent study showed that vancomycin-treated mice had little weight change and no improvement in glycemic control ${ }^{296}$, and consistent with the presented data, a 4-day treatment with a broad-spectrum antibiotic cocktail did not affect postprandial glucose metabolism in lean healthy $\operatorname{men}^{297}$. Furthermore, it has recently been shown in a limited number of obese subjects with the metabolic syndrome that vancomycin slightly but significantly reduced peripheral insulin sensitivity, despite comparable changes in microbial composition and bile acid metabolism as observed in the present study ${ }^{80}$. Although the data of the latter study seems at odds with the present findings, it is important to emphasize that the study by Vrieze and colleagues ${ }^{80}$ lacked a placebo group and therefore the modest ( 4\%) vancomycin-induced decrease in peripheral insulin sensitivity was based on a within group comparison (post-treatment versus pre-treatment). Additionally, follow-up measurements that were performed eight weeks after treatment cessation in the present study also did not show an effect on wholebody insulin sensitivity, despite a still considerably altered microbial composition as compared to pre-treatment as well as placebo. The present study, which was well-powered and placebo-controlled, indicates that the previously reported vancomycin-induced effects on peripheral insulin sensitivity are probably of minor physiological significance.

Chapter 5 examined the association of the microbiota and tissue specific insulin sensitivity in two independent cohorts of overweight and obese pre-diabetic male subjects, from the Dutch regions of Maastricht and Amsterdam. Although a growing body of evidence suggests that human gut microbiota plays a role in the development of obesity and related metabolic diseases, there is little consensus on biomarker organisms between human studies, which could be due to biological as well as technical reasons or a combination thereof ${ }^{47,} 314$. In addition, no human data are available that investigated whether tissue specific insulin sensitivity, as measured by the golden standard hyperinsulinemic-euglycemic clamp technique, is related to a specific microbial pattern. In all subjects, hepatic, peripheral and adipose tissue insulin sensitivity were determined by a two-step hyperinsulinemic-euglycemic clamp with $\left[6,6-{ }^{2} \mathrm{H}_{2}\right]$-glucose tracer infusion. Remarkably, despite the fact that both cohorts were constructed on comparable recruitment strategies, the average microbiota composition in both cohorts showed pronounced differences.

This study, initially set up to merge two very well defined cohorts and to mine the data for host-microbe associations, revealed important yet distinct observations, both related to 
the conclusions from chapters 2 to 4 . Firstly, we found no consistent and significant association between liver, adipose tissue or skeletal muscle insulin sensitivity and the microbiota composition of insulin resistant, yet non diabetic, obese males in either of the cohorts, except for a very strong Spearman correlation of 0.57 between Peptococcus niger et rel. Nevertheless, Random Forests classifiers using microbiota composition as predictors revealed taxa associated with fasting glucose concentrations and HbAc1 but only in one cohort. The top microbial features distinguishing classes were different Proteobacteria and Faecalibacterium prausnitzii, Roseburia intestinalis, Clostridium nexile and Eubacterium rectale and related species for fasting glucose levels. For $\mathrm{HbAc1}$ these taxa were Oscillospira guillermondii, Sporobacter termitidis, Lactobacillus gasseri and Peptococcus niger and related species. The striking cohort-specific observations suggest that the relation between microbiota composition and T2DM as well as other characteristics of the metabolic syndrome is very dependent on the selected cohort of patients and their respective baseline microbiota composition. Similar observations have been made by other researchers as well.

Nevertheless, it is possible that differences in microbiota composition are not associated with the insulin resistance phenotype when the overweight and/or obese state of the patient is already established, as is the case for our metabolic syndrome patients. In the latter case we cannot exclude that the composition of the fecal microbiota may play a role in the worsening of insulin sensitivity in an early stage in the development from a lean towards an overweight/obese phenotype. Furthermore, the observation of a subgroupspecific microbiota only observed in one of the cohorts might indicate an alternative state of microbiota composition driven by yet unknown forces. Nevertheless, this study clearly demonstrated that cohort-specific microbiota differences hamper finding a consensus biological interpretation between cross-sectional studies. This, combined with the complexity of individual disease pathogenesis, as well as the individual-specific differences in microbiota composition, may explain the inconsistency in observations between different studies concerning the identification of signature microbes for obesity, inflammatory bowel disease, irritable bowel syndrome and other diseases ${ }^{325-327}$.

Chapter 6 describes a bioinformatics pipeline for the data analysis of the currently most widely used format to profile microbial ecosystems. Massive high-throughput sequencing of short, hypervariable segments of the 16S ribosomal RNA (rRNA) gene has transformed the methodological landscape describing microbial diversity within and across complex biomes. However, several studies have shown that methodology rather than the biological variation is responsible for observed sample composition and distribution ${ }^{343}$. The impacts of some of these technical aspects are so substantial that they compromise true metaanalyses. Currently there is a lack of consensus regarding key features of the healthy microbiome, such as richness, diversity and composition, while the link with specific human pathologies often reveals study-specific sets biomarker organisms ${ }^{47}$. Large metaanalyses would aid in elucidating whether the basis for these differences is biological, technical or maybe a combination of both. To facilitate these meta-analyses of microbiota 
studies we developed NG-Tax, a pipeline for 16S rRNA gene amplicon sequence analysis that was validated with different mock communities. We used a setup that allowed for the evaluation of important factors of technical bias in taxonomic classification: 1) run-to-run sequencing variation, 2) PCR - error, and 3) region/primer specific amplification bias. Despite the short read length $(\sim 140 \mathrm{nt})$ and all technical biases, the average specificity of the taxonomic assignment for the phylotypes included in the mock communities was $96 \%$. On average $99.94 \%$ of the reads could be assigned to at least family level, while assignment to 'spurious genera' represented on average only $0.02 \%$ of the reads per sample. The analysis of $\alpha$ - and $\beta$-diversity confirmed conclusions guided by biology rather than the several methodological aspects. In conclusion, NG-Tax demonstrated high robustness against choice of region and other technical biases associated with 16S rRNA gene amplicon sequencing studies, diminishing their impact and providing accurate qualitative and quantitative representation of the true sample composition, which will improve comparability between studies and facilitate efforts towards standardization.

Chapter 7 illustrates the application of the pipeline and recommendations from chapter 6 to biological samples with the aim to characterize dynamics of microbial community composition and global activity in an in vitro gut model (TIM-2) fed either with a normal diet, or modified versions from which the carbohydrate (MPLC) or protein fraction was diluted (LPMC) for $72 \mathrm{~h}$. The production of short chain fatty acids and branched chain fatty acids reflected the quantity of carbohydrate and protein in the system. The developing communities were monitored through 16S ribosomal RNA gene sequencing of two variable regions, and overall, each diet produced distinct microbial communities and temporal patterns. The microbiota in reactors fed diets containing normal carbohydrate levels were enriched in members of the genera Prevotella, Subdoligranulum, Blautia and Bifidobacterium, all associated with carbohydrate fermentation. In turn, the microbiota in the reactors fed the MPLC diet, containing ten-fold less carbohydrates, was enriched in the genus Bacteroides, which is associated with diets rich in protein and animal fat. These observations of distinct communities were corroborated at a global functional level by analysis of the metatranscriptome. This setup allows researchers to study the (trophic) interactions and task division within a community and how these are impacted by diet-related factors under controlled conditions. This may assist in defining causal links between specific diet-derived parameters, microbial groups and their activities.

\section{Universality of human microbial dynamics}

Chapters 2 t/m 6 largely explore the association between the microbiota and features of metabolic syndrome. What stands out is the overall lack of consistency regarding the identified microbial biomarkers and putative mechanisms underlying the observations Furthermore, the antibiotics intervention described in chapter 4 represents an example of the contrast between mouse studies and human interventions. Studies have indicated that antibiotic treatment may improve glucose homeostasis and metabolic impairments in rodents $^{265-267,293-295,400}$, whereas the study presented in chapter 4 indicates that 
interference with adult microbiota by antibiotic treatment for 7 days had no clinically relevant impact on metabolic health in obese humans. This could be due to more extreme interventions, as a result of less stringent ethics requirements and the low microbial and genetic variability associated with rodent studies, which help to create a relatively strong and clean biological signal. In humans however, this signal might be dampened by more heterogeneous responses (chapter 1).

Contrasting observations between studies and subsequently the inability to identify a consistent microbial group has not only been observed in metabolic syndrome, but also in other comparative studies between healthy individuals and those suffering from disorders such as IBS ${ }^{47,52,314}$. In many cases this might be attributed to multiple biological factors, such as differences in diet, age, BMI and gender between cohorts (chapter 2), as well as methodological variation (primers, reference databases, analysis pipelines; chapter 6). Walters and colleagues have shown that using the microbiota, individuals could be classified as lean or obese with statistically significant accuracy within a study, consistent with the ability to experimentally transfer this phenotype in mice by transfer of the corresponding microbiota. However, microbial signatures were not consistent between studies even when the data was analysed in the same way ${ }^{148}$. Moreover, chapter 5 compared two very homogenous cohorts with regards to the (pathological) host phenotype, again using the same methodological platform (DNA isolation, primers, microarray and analysis pipeline) and still found very profound differences in the composition of the microbiota, seemingly not related to the very well-defined and distinctive phenotype (overweight, pre-diabetic males). As a result, discrepancies in associations between these host characteristics and the microbiota were observed. Although the differential microbial biomarkers of T2DM in different cohorts have been identified previously ${ }^{314}$, those compared groups were arguably much more different; Danish women in varying states of insulin resistance and Chinese individuals with $\mathrm{T}_{2} \mathrm{DM}^{195,314}$. The groups in this thesis were far more similar (prediabetic Dutch males), yet the observations are analogous.

In chapter 5 none of the previously mentioned variables known to influence the microbiota, such as age, BMI, diet and gender, could explain the difference in composition of the microbiota between the two cohorts. As outlined in chapter 1 the interplay of variables that give rise to the variation of the microbiota is complex and is not yet fully understood. The difference between the two cohorts suggests a latent or unknown parameter, for instance lifestyle parameters linked to geographical location, driving the composition and overpowering all measured variables, which failed to explain much of the variation in microbiota composition. A similar observation was made in chapter 3, where weight related parameters explained very little of the total variation, again contrasting animal and some human studies ${ }^{48,62}$.

If we would compare the microbiota of both cohorts in chapter $\mathbf{5}$ independently with one cohort of healthy individuals, the "dysbiotic" state would be different in both groups. However, the question of what a normal healthy microbiota actually entails has not been 
answered satisfactory yet (chapter 1), and Walters and colleagues also showed that the composition of the healthy control group can be different between studies ${ }^{47}$. Therefore, comparative analyses between different patients and healthy cohorts could lead to just as many 'dysbiotic' states or sets of microbial biomarkers.

The previous paragraph invokes three important questions. 1. Does a "normal" healthy microbiota exist, what is its composition and how large is the variation?, 2. Is the composition truly linked to a given specific phenotype that is studied or some other "hidden" variables associated with that cohort? Or alternatively, 3. Is the dysbiotic state truly group-specific, or even individual-specific, and are we measuring these differences based on a grouping because they live in geographical proximity?

To answer these questions, we need to know which factors actually shape our microbiome, how does it develop (chapter 1) and what are the rules (if there are any) that govern a normal functioning of this ecosystem. We know that the microbiota is highly individual, and data suggest that it is relatively stable (except for variations in abundance) in adults in the absence of gross perturbation. Nevertheless even the adult gut composition appears to change slightly over time with regards to its members ${ }^{9,42,43}$. These, routine, mild fluctuations in community composition in the absence of gross disturbance indicate that long-term stability of human gut microbial communities is not maintained by inertia, but rather by the action of restoring forces within a dynamic system ${ }^{44}$. In other words, are there any fundamental universal ecosystem 'rules' that drive it towards this equilibrium, or is the microbiota of any individual an island with a unique ecosystem regarding the interplay of the microbiota with itself as well as with the host? More concisely, are these rules largely host-independent (that is, universal) or host-specific, or lies the truth in a combination of both?

The methodological obstacles on which we elaborated in chapter $\mathbf{6}$ are commonly one of the most significant drivers of variation in microbiome studies and might explain some but not all the inconsistencies found in literature ${ }^{343}$. If we disregard these and focus purely on the underlying biological principles, there are three hypothetical fundamental cases regarding the dynamics of the microbiota.

The microbiota has either: 1 . individual dynamics, in which all the ecological parameters are different in different subjects, 2. group dynamics, in which subjects can be classified into groups based on certain host factors which share the same ecosystem properties, or 3 . universal dynamics, in which all individuals share the same set of ecological parameters ${ }^{401}$ (Figure 1). Addressing these alternative working hypotheses is vital for the comprehension and interpretation of data presented in this thesis as well as literature in general, but also for the development microbiome-based therapies. If the dynamics are indeed universal, the interpersonal variability stems solely from the different assemblages of colonizing species in different individuals. General interventions can be designed to control the microbial state (in terms of species assemblage and abundance profile) of different individuals. By contrast, if the dynamics are strongly host-specific, truly 
personalized interventions need to designed, which need to consider not only the unique microbial state of an individual but also the unique dynamics of the underlying microbial ecosystem.

One very important remark is that that host or group specific dynamics could extend to the host-microbe interaction, thus every person reacts differently to certain bacteria as in vitro studies have shown a remarkable donor effect in in vitro assays ${ }^{402}$. Chapter 1 describes that the formation of the microbiota is highly individual and dependent on many stochastic effects. Therefore, it is very likely that during these early life exposures our immune system is trained with a unique set of bacteria. This could raise major safety concerns in for instance FMTs because although the healthy microbiota is stable in the donor's gut, it may be shifted to an undesired state in the recipient's gut ${ }^{401}$.

a
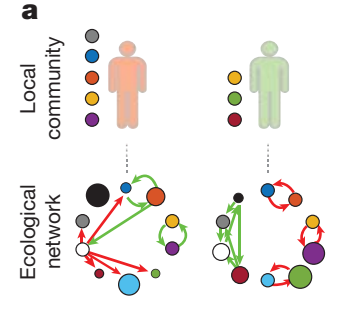

Individual dynamics
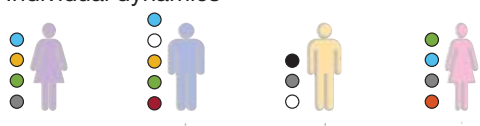

b
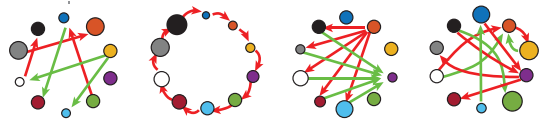

Group dynamics
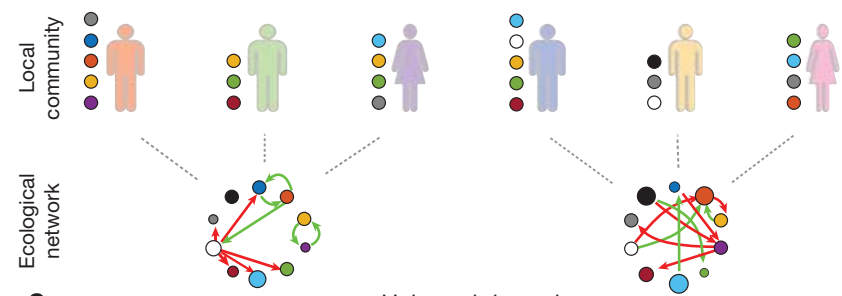

c

Universal dynamics
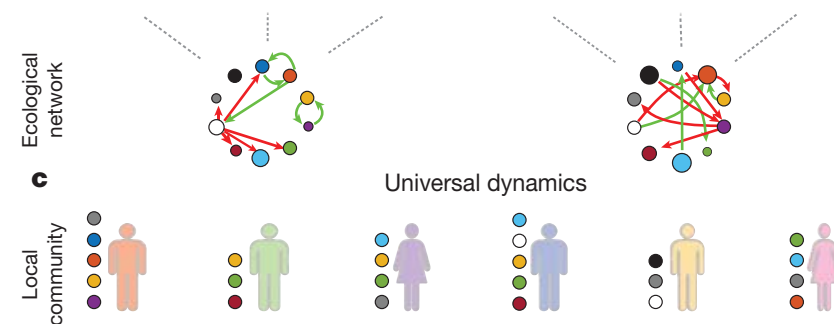

-11

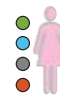

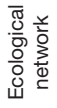
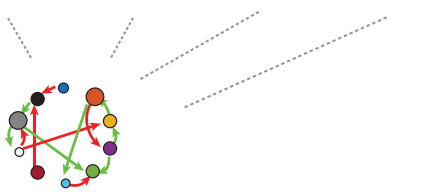

Figure 1. Alternative scenarios of microbial dynamics across different healthy subjects ${ }^{401}$

Recently Bashan and colleagues addressed this question of universality through an indirect approach. Ideally, long and densely sampled time series from many individuals with different traits and backgrounds should be studied. However, because such large datasets are currently lacking, they measured two independent aspects of community similarity, i.e. overlap and dissimilarity. Overlap compares species assemblies by quantifying the proportion of shared species, whereas dissimilarity assesses the difference 
in abundance profiles of the shared species between individuals. Plotting overlap against dissimilarity they could visualize whether communities that shared more species, showed a higher compositional similarity ${ }^{401}$. If microbiota dynamics were to be truly universal (host-independent), then having the same species present should lead to the same relative proportion of those species, because they would dynamically influence each other in the same way ${ }^{403}$. The authors applied this approach to cross-sectional data from two large-scale metagenomic studies and showed that gut and mouth microbiomes display pronounced universal dynamics, whereas communities associated with certain skin sites are probably shaped by differences in the environment of the host. Nevertheless, this analysis was based on a number of assumptions. First, that the microbiota is in a steady state and that having the same steady state implies that the microbiota is governed by the same dynamics. Although it seems that the microbiota is not in a steady state per se, but rather under the action of restoring forces within a dynamic system, driving it towards an equilibrium ${ }^{44}$, one might assume that with enough samples the relatively small intra-individual effects will be cancelled out, therefore making this a reasonable assumption. An alternative explanation for their observations regarding the universality of gut and mouth microbiomes of healthy individuals, could be that some host (environmental) factors not only select for the presence of certain microbes but also drive their relative abundances by enforcing certain optimally adapted compositions. According to this model, the microbiota may end up in similar steady states not because of their intrinsic dynamics (as suggested for instance for the enterotypes ${ }^{92}$ ), but because of a strong environmental pressure that may shape gut microbial communities. As the authors rule out obvious confounders such as long-term dietary pattern, BMI, age and stool consistency, they do not account for all factors that may possibly influence the gut microbiota, and hence cannot provide an entirely conclusive answer regarding the universality of the gut's microbial community dynamics ${ }^{403}$.

Nevertheless, if these assumptions hold than the aforementioned observations point to universal gut microbial dynamics in healthy individuals. Interestingly, the gut microbiota of patients recurrently infected with the bacterial pathogen $C$. difficile did not display the characteristics of universal dynamics, while they regained these after faecal transplantation from healthy donors. The lack of universal dynamics in a clearly dysbiotic state such as a $C$. difficile infection and the restoration of universal dynamics after transplant might point to individual or group dynamics in circumstances where the microbiota is disturbed. This seems to be in line with the conclusions from chapter 2-5 that show very little overlap in identifying human (over)weight related microbial biomarkers.

\section{Non-universal dynamics in disease}

Although some pathological conditions such as IBD have shown to be associated with a consistent biological signal ${ }^{47}$, there is a lack of a consensus panel of microbial biomarkers for a range of other conditions, such as $\mathrm{IBS}^{73}$ and $\mathrm{OAMD}^{47}$ (chapter 2) and it is tempting 
to consider that indeed in certain diseased states the dynamics are not universal, but individual or group specific at least in some cases. Nonetheless, if the rules for a healthy microbiota are universal we need to define what is normal and healthy and try to deliberately direct/manipulate the microbiota in a certain direction with interventions. Although understanding the factors that lead to alterations in composition of the microbiota in these conditions is still informative, currently it seems to be more beneficial to understand what is normal in order to define deviations from that normal: namely dysbiosis. Because it seems that at the moment our knowledge on the interplay and relative importance of factors shaping the microbiota under different circumstances is lacking. The two cohorts from Maastricht and Amsterdam described in chapter $\mathbf{5}$ are surprisingly very different regarding gut microbiota composition despite the fact that the subjects were recruited similarly. Currently, we do not know what the underlying mechanisms are that cause this difference.

\section{Group dynamics}

If group dynamics exist within human subjects we need to identify the grouping parameters, because now cohorts can be different regarding microbiota composition due to unknown or 'hidden' host variables. Some of these variables might not even be biological but caused by technological variation ${ }^{343}$. True meta-analyses with the aid of analysis pipelines that are robust against technological biases associated with microbiota profiling using PCR amplification of the 16S rRNA gene (chapter 6), will aid in the discrimination between biological and technical factors. Case control studies will depend on universal healthy control groups, in case these exist, to determine the extent of compositional and/or functional dysbiosis.

\section{Individual dynamics; highly individual disease phenotypes}

It has been proposed that the quote "Every unhappy family is unhappy in its own way" from Tolstoy is a metaphor for the individual nature of human disease ${ }^{404}$, suggesting that complex human diseases are large collections of individually rare genetic conditions, epistasis (interaction between genes) and penetrance (the proportion of people with a particular genetic change who exhibit signs and symptoms of a genetic disorder). The latter is probably the result of a combination of genetic, environmental, and lifestyle factors, many of which are unknown. Combined with the inter-individuality of the microbiome and the influence of genes whose mutations are known to be associated with gastrointestinal pathologies as well as with differences in microbiota composition, such as $\operatorname{Nod} 2^{41}$, and $F U T 2^{40}$, it is probably safe to assume that the search for common biological signals is at least challenging if not impossible. In light of the multifactorial pathogenesis of human disease the manifestation of individual dynamics of the microbiota seems plausible and even highly likely.

\section{Approaching health as a result of host-microbiome ecosystem services}

At the global scale humans benefit from a variety of processes supplied by natural ecosystems, such as provisioning services (food, water and raw materials), regulating services which are benefits obtained from the regulation of ecosystem processes (carbon 
sequestration, climate regulation, waste decomposition and detoxification) as well as supporting services that are necessary for the production of all other ecosystem services (nutrient recycling, soil formation). Collectively, these benefits are known as ecosystem services $^{405}$. There is growing evidence that human health is a collective property of the human body and its associated microbiome, and thus could be considered a net effect of ecosystem services ${ }^{31}$.

Costello and colleagues envisaged a more holistic view on clinical medicine focused on managing our microbiome (and ourselves) to preserve these ecosystem services. In general, a theoretical ecology framework is applied for the optimal management of these ecosystem services. 1. Identify the ecosystem services (ES) and their providers (ESPs, ecosystem service providers) and characterize their functional roles. 2. Determine how community context influences the function of these providers as well as the ecosystem as a whole. 3. Assessment of key environmental factors influencing the provision of services. 4. Measure the spatial and temporal scales at which these providers and their functions operate. If studies of the human microbiome were structured around these four priorities, the development of an ecological approach to medicine could be accelerated ${ }^{31}$.

The vast majority of research on the human GI microbiota is still focused on identifying ESPs through associations. Nevertheless, in the last decade all four areas have seen enormous progress, mainly through studies that applied a more ecosystems approach with an intervention and the omics toolbox to answer specific questions regarding the underlying mechanisms at the level of composition, activity and metabolic output of the microbiota as well as the effects on the host. Table 1 shows a selection of these studies and their contributions to the respective priorities. ES such as a role in host energy and lipid metabolism ${ }^{48,103,406}$ and ESPs (biomarker organisms) have been identified, and causality has been demonstrated in rodent studies for instance for Bifidobacterium ${ }^{407}$, Akkermansia muciniphila ${ }^{65}$ and Christensenella minuta ${ }^{37}$. Nonetheless, all these have not been validated in humans yet. Area 2 and 3 (community context and key environmental factors influencing ESPs and the provision of ecosystem services) have mainly been studied using gene knockout mouse models and gnotobiotics ${ }^{408,409}$. Area 4 (the spatial and temporal scales at which ES and ESPs operate) has been studied using interventions and measuring the subsequent metabolic output focused on specific metabolites (such as SCFA or genotoxic compounds) and preferentially their direct or long term effects on the host (chapter 4) ${ }^{399}$. These types of studies, structured around one or more of these four priorities, have deepened our understanding of the microbiome. 


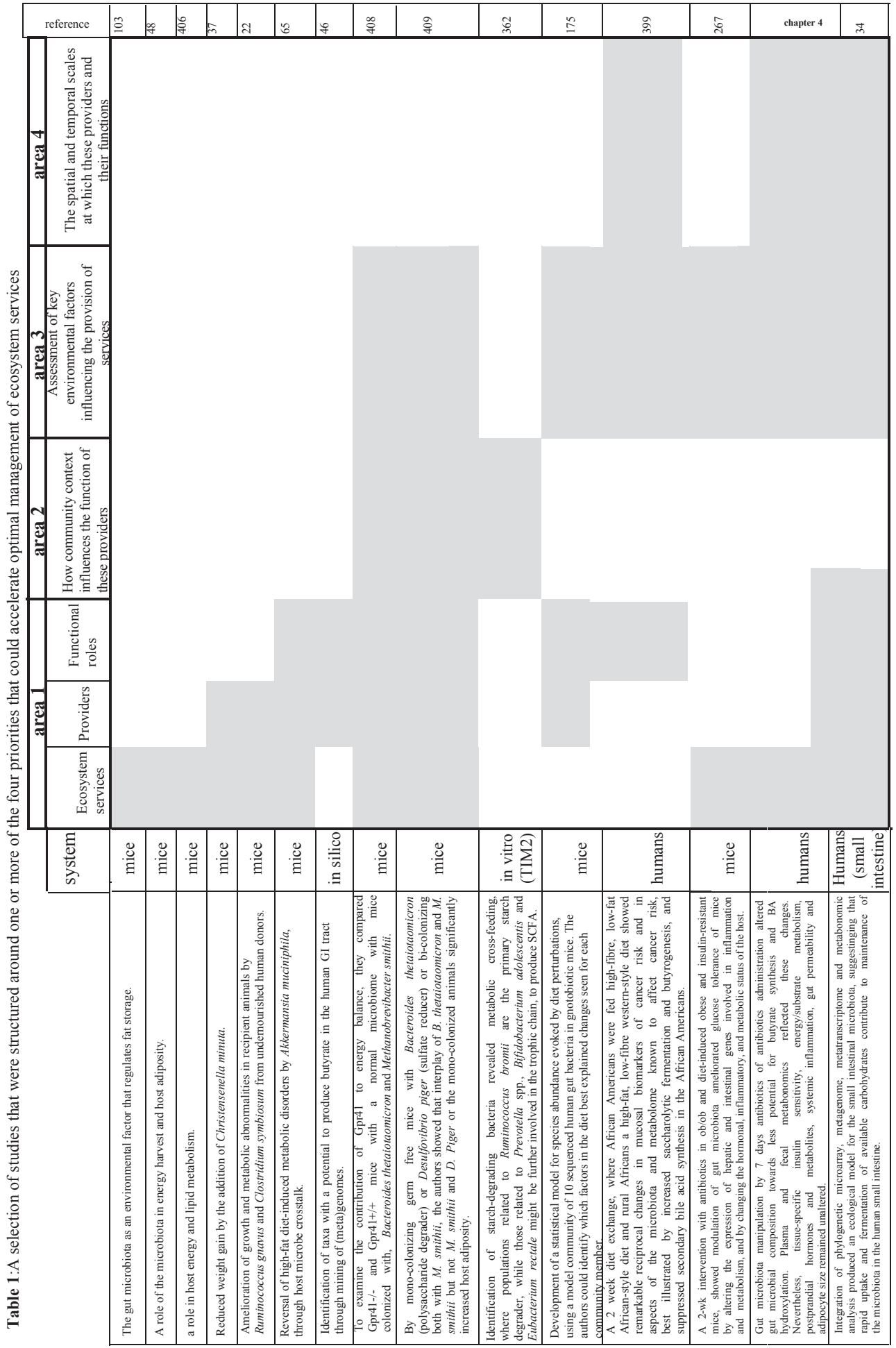




\section{Challenges ahead}

Although large improvements have been made and the steps for the management of ecosystem services are relatively logical and straightforward, in practice some factors specific for microbial ecosystems are currently hampering progress. These challenges should be prioritized for the field to move forward.

\section{Microbial function}

In most forms of host pathology with which the microbiota has been implicated, the concept of "dysbiosis" or 'community as pathogen' has been proposed. This is a clear distinction between the more traditional medical concepts for infectious disease in which a single virulent and/or dominant microbial species acts as the pathogenic factor. Human intervention but also interactions between community members may underlie the critical features associated with some of these pathologies ${ }^{44}$. Understanding the function and activity of these partners is critical for the identification of underlying mechanisms. This, however, is still frustrated by the large fraction of uncultured organisms and many genes that lack annotation. ${ }^{410}$ This might equally apply to ESPs themselves, as due to the potential of complex interactions between partners, even a cultured ESP might only perform its specific function with the aid of an uncultured partner. Although there are indirect means to assign functions to uncultured microbial entities by binning coabundant genes from metagenomic sequence data into metagenomic species (MGS) ${ }^{7}$, many of these genes will still lack functional characterization, due to the absence of known homologues in databases ${ }^{410}$. Functional metagenomics, i.e. screening of libraries or direct cloning of sequences in heterologous expression hosts combined with various functional screening assays, although laborious, might currently be the only solution. However, the throughput of these assays will need to be increased to make this a viable solution. Nevertheless, there is only so much we can learn from studying these complex communities as a whole and there is a growing need to know more about ESPs. Therefore, we also still need to culture isolates ${ }^{411}$. These two approaches to characterize function, individuals and whole community analysis, are complementary and provide the basis for the generation of hypotheses and find answers regarding human GI ecosystem services. 


\section{Microbial composition}

The currently most popular technique to profile microbiota composition is based on amplification of the 16S rRNA gene. Although, this type of data is relatively simple, it lacks resolution, which makes interpretation difficult. This is partly because phylogenetic classification beyond genus level, based on the short read length of the current sequencers, should be treated with great caution. The following serves to emphasize the potential genetic variation that is still present at this phylogenetic level. For instance the gene content of strains within a species can differ up to $30 \%{ }^{412}$ and the shared genome size of the virulent E. coli $\mathrm{OH} 157$ and the intestinal isolate E. coli $\mathrm{K} 12$ was determined to be only $857 \mathrm{~Kb}^{413}$. Fascinatingly, this species even contains a strain that is in use as a probiotic, E. coli strain Nissle $1917^{414}$.

Another complicating factor is the high functional redundancy in the GI microbiota. This redundancy enhances resilience, thus ensuring the continuity of functions that are important to the host ${ }^{44}$. However, this also means that different compositions are able to perform the same essential functions, such as the production of butyrate ${ }^{46}$. Therefore, in case control studies that only apply composition profiling, the actual functional output of a system presumed to be in 'dysbiosis' might not be abnormal and vice versa; lack of significant differences in abundance doesn't necessarily indicate a healthy state. The latter was accentuated in a study from O'Keefe and colleagues. After a dietary intervention microbiota did not show large compositional differences yet the metabolic output changed significantly, including the abundance of important bacterial metabolic risk factors for colon cancer $^{399}$.

\section{Future perspectives}

From the last paragraphs it is clear that the ultimate way forward would be integrative 'ecosystems biology' approaches like proposed by Zoetendal and colleagues ${ }^{34}$ that combine several complementary omics technologies, supplemented with physiological host data, preferably sampled in (dense) time series, with proper biological controls, such as routine temporal sampling at baseline to determine normal fluctuations and using crossover designs. Another improvement over current studies would be power calculations based on microbiome data instead of host variables ${ }^{415}$.

A common theme in this chapter and chapter 1, is the need for large densely sampled temporal datasets of many individuals with diverse backgrounds and life styles from various geographical locations, thus with different exposures and genotypes. These would assist in the elucidation of fundamental mechanisms regarding (universal) ecosystem rules in health as well as disease and the stability and resilience of the normal healthy microbiome. It might also reveal clues about cause consequence and the temporal dynamics of dysbiosis, i.e. whether this process is abrupt and comparable to a stability landscape 
where repeated perturbations knock a system from one state into another or whether changes are gradual with a linear trend into dysbiosis. Different layers of data based on DNA, RNA and proteins from the side of the microbiome will give clues whether dysbiosis is merely functional, compositional or both. Simultaneous assessment of the mediators of reciprocal host-microbe interaction (microbial metabolites and immunological parameters) might identify causality; what changed first and who influenced whom at what point in time.

Although only a few years ago it would have seemed impossible to generate and analyse such datasets, they are now within our grasp as a result of the revolutionary advances in genomics and associated technologies, computational biology as well as the reductions in sequencing costs. Probably the main obstacle would the considerable burden this would place on participants. However, just sequencing more is not the answer. This is true for compositional profiling, but for functional analyses as well. Chapter 6 has shown the relatively high noise levels associated with 16S rRNA gene amplicons sequencing. Therefore, analysis pipelines must continually improve to deal with the new quantities and types of data. Furthermore, only recently, common requirements of any scientific endeavour, namely proper controls, are now commonly integrated to discern between potential technological artifacts and biology. These controls include negative controls for samples with low amounts of bacterial DNA ${ }^{16}$, the inclusion of Mock Communities of reasonable complexity (chapter 6), preferably related to the ecosystem of interest, and other approaches such as spiking in 16S rRNA gene amplicons to more accurately determine abundance ${ }^{416}$. Also current community analysis would greatly benefit from cleaner reference databases. Furthermore, functional analyses based on the genome, transcriptome and proteome of the microbiome would benefit from efforts to functionally characterize the multitude of genes that remain without characterized homologues in the databases.

Animals and especially gnotobiotic mice, are considered the ideal model system to generate hypotheses and gain insight into putative mechanisms because they are physiologically comparable to humans. Researchers can generate relatively clean biological signals and very homogeneous responses due to fewer ethical constraints on the severity of the intervention. Nonetheless, these results have been shown to be difficult to replicate in humans. When the time is there to move beyond hypothesis and translate the results of animal experiments to humans, studies need relatively harsh interventions (for instance dietary, antibiotic or FMT) to induce a quantifiable change, because of the dampening effect of differences in inter-individual responses due to the heterogeneity of human populations regarding the composition of the microbiome, genotype, phenotype, life style and the effect of other environmental variables which are known to influence the microbiota. Therefore, human microbiome research requires intelligent approaches to obtain a biologically relevant signal. Dietary interventions do not need (unethically) 
extreme amounts of macronutrients, but might be based on previous long term dietary habits, such as a switch between a diet rich in fiber to a diet rich in fat and protein and vice versa $^{399}$. Or alternatively, an intermediate step might be introduced between the current mice studies and validation in human populations. For instance, if results can be replicated in an experimental setup with less homogeneity regarding the aforementioned parameters, such as wild mice, the next step could be validation in a human population.

\section{Paradigm shift towards an ecological approach to human health}

The study of the human microbiome is still in its infancy. Nevertheless, we have already started to recognize the critical and integral role of the microbiome in our health and wellbeing. This perspective, i.e. the 'outside' is aiding the 'inside', is relatively new and in great contrast to the more traditional clinical view of the human body and microbes. This body-as-battleground approach ignores the community context of infectious disease, and does not take into account our increasing knowledge regarding the assembly of the human microbiome. Given the ecological parallels between assembly of the human microbiome and assembly of other ecological communities, Costello and colleagues suggested an approach to human medicine that has more in common with park management, than it does with battlefield strategy ${ }^{31}$. Human intervention such as antibiotics use has shown deleterious effects on the microbiome and human health, and at a global scale we have also recently started to realize that human intervention has irrevocably changed and disrupted delicate ecosystems. Therefore, we can draw parallels between the GI microbiome and various other habitats, including plant and animal communities in highly disturbed environments impacted by overfishing, (abrupt) climate change, habitat loss, ocean acidification, pollution, and invasive species and learn from the approaches that have been successfully applied to manage biodiversity in dysbiosis on a much grander scale. The effective management of plant or animal communities requires a multifaceted approach of habitat restoration, promotion of native species, and targeted removal of invaders. Currently, it is increasingly recognized that the traditional 'one-size-fits-all' approach to medicine that is based on broad population averages is often not effective due the heterogeneity in genotype and phenotype of human populations and the fact that it is not fully understood how people develop disease and respond to treatments. To this complex web of interactions (epistasis and penetrance) we can add the microbiome, not only because of its intimate relation with our health but also due to a direct effect on treatment efficacy trough differential metabolization of medication ${ }^{417}$. Tailoring health care to diagnostic changes in an individual's microbiome and each person's unique genetic makeup will move us closer to more precise and predictable health care. Our growing understanding of the microbiome and human genetics and how they drive health, disease and drug responses in each person will enable doctors to provide better disease prevention and more effective treatments for human diseases. 


\section{Summary}

After birth, our gastrointestinal (GI) tract is colonized by a highly complex assemblage of microbes, collectively termed the GI microbiota, that develop intimate interactions with our body. Recent evidence indicates that the GI microbiota and its products may contribute to the development of obesity and related diseases. This, coupled with the current worldwide epidemic of obesity, has moved microbiome research into the spotlight of attention. Although the main cause of obesity and its associated metabolic complications is excess caloric intake compared with expenditure, differences in GI tract microbial ecology between individuals might be an important biomarker, mediator or even new therapeutic target. Nevertheless, it is currently still unclear which bacterial groups play a role in the development of the metabolic syndrome, in humans. This might partly be explained by: 1 . Biological factors such as the heterogeneity in genotype, lifestyle, diet; and the often complex aetiology of human disease of which the metabolic syndrome is no exception. 2 . Technological factors, such as the use of miscellaneous incompatible methods to assess the gut microbiota, often enumerating specific groups rather than using broad 16S rRNA gene surveys or metagenomics. 3. Studies vary greatly in the populations considered, their designs, and the degree of control for potential confounding factors such as lifestyle and diet. Nevertheless, recent research on this matter has shown a conceptual shift by focusing on more homogenous subpopulations, based on stricter control over variables such age range or through the use of both anthropometric (weight, total body fat) as well as biochemical variables (insulin resistance, hyperlipidaemia) to define groups.

Perturbations in microbial diversity and community structure in adults with overweight and obesity may be partly due to long-term dietary habits or physiological changes in these subjects. As such, exploring the association between the gut microbiota and variation in BMI and weight in early life, prior to or close to the onset of overweight, might provide additional insights into these processes. Therefore, we studied the fecal microbiota of 295 six-seven year old children from the KOALA Birth Cohort, living in the south of the Netherlands. This age range is relatively uncharted microbiota territory. We found that its composition seems to conform to tot same ecosystem rules as that of adults. The bimodal distribution pattern of several bacterial groups as well as their co-correlating groups that were reported previously, including Uncultured Clostridiales II, Prevotella spp. and Dialister were confirmed. Furthermore, one of the previously described bimodal groups (Uncultured Clostridiales I) was shown before to exhibit very clear shifting state probabilities associated with ageing, where the high abundance state was mainly observed above 40 years of age. This was corroborated as no support for bimodality of this group was observed in the children included in the study described here. A large part of the variation in microbiota composition was explained by the abundance of aforementioned groups in contrast to the anthropometric outcomes, suggesting that in this group of healthy 
children within a relatively normal weight range, weight and associated parameters were not major drivers of overall genus-level microbial composition or vice versa. Hereafter, multiple linear and logistic regression models with rigorous adjustment for confounders were applied to investigate individual microbiota features association with weight related anthropometric outcomes. Previously reported parameters such as diversity, richness and Bacteroidetes to Firmicutes ratio, were not significantly associated with any of the outcomes. Nevertheless, the abundance of several specific bacterial taxa; Akkermansia, Sutterella wadsworthia et rel. and Bryantella formatexigens et rel. and the dichotomous abundance state of the bi-modally distributed UCII was consistently associated with weight-related outcomes.

Other biochemical features of the metabolic syndrome have been associated with the gut microbiome. Mainly rodent studies have indicated that antibiotic treatment may improve glucose homeostasis and metabolic impairments. Therefore, the effects of gut microbiota manipulation by antibiotics ( $7 \mathrm{~d}$ administration of amoxicillin, vancomycin or a placebo) on tissue-specific insulin sensitivity, energy metabolism, gut permeability and inflammation in 57 obese, pre-diabetic men from the same geographical region, were investigated. Vancomycin decreased bacterial diversity and significantly reduced well known butyrateproducing Firmicutes from Clostridium clusters IV and XIVa and bacterial groups involved in bile acid metabolism. These changes occurred concomitantly with altered plasma and fecal concentrations of these metabolites. In adipose tissue, gene expression of oxidative pathways was upregulated by antibiotics, whereas immune-related pathways were downregulated by vancomycin. However, antibiotic treatment had no significant effects on tissue-specific insulin sensitivity, energy/substrate metabolism, postprandial hormones and metabolites, systemic inflammation, gut permeability and adipocyte size. Importantly, despite a still considerably altered microbial composition at eight weeks follow-up, energy harvesting, adipocyte size and whole-body insulin sensitivity (HOMA-IR) remained unaltered. Overall these data indicate that interference with adult microbiota by antibiotic treatment for 7 days had no clinically relevant impact on metabolic health in obese humans. These data are in contrast with several rodent studies as well as a human intervention. The present study, which was well-powered and placebo-controlled, indicates that the previously reported vancomycin-induced effects on human peripheral insulin sensitivity are probably of minor physiological significance.

The aforementioned group that was relatively homogeneous with regards to phenotype was combined with another cohort with similar phenotypical characteristics (obese, male and pre-diabetic) from another region of the Netherlands, to investigate whether tissue specific insulin sensitivity, as measured by the golden standard hyperinsulinemic-euglycemic clamp technique, is related to a specific microbial pattern. Remarkably, despite the fact that both cohorts were constructed based on comparable recruitment strategies, the average 
microbiota composition in both cohorts showed pronounced differences. Firstly, we found no consistent and significant association between liver, adipose tissue or skeletal muscle insulin sensitivity and the microbiota in both cohorts. Nevertheless, Random Forests classifiers using microbiota composition as predictors revealed taxa associated with fasting glucose concentrations and $\mathrm{HbAcl}$ but only in one cohort. The top microbial features distinguishing classes were different Proteobacteria and groups involved in butyrogenesis, such as Faecalibacterium prausnitzii, Roseburia intestinalis, and Eubacterium rectale and related species, for fasting glucose levels. For $\mathrm{HbAc1}$ these taxa were Oscillospira guillermondii, Sporobacter termitidis, Lactobacillus gasseri and Peptococcus niger and related species. The striking cohort-specific observations suggest that the relation between microbiota composition and type 2 diabetes mellitus as well as other characteristics of the metabolic syndrome is very dependent on the selected cohort of patients and their respective baseline microbiota composition. Similar observations have been made by other researchers as well. It could be that differences in microbiota composition are not associated with the insulin resistance phenotype when the overweight and/or obese state of the patient is already established, as is the case for our metabolic syndrome patients. In the latter case we cannot exclude that the composition of the fecal microbiota may play a role in the worsening of insulin sensitivity in an early stage in the development from a lean towards an overweight/obese phenotype. Furthermore, the observation of a subgroupspecific microbiota only observed in one of the cohorts might indicate an alternative state of microbiota composition driven by yet unknown forces. Nevertheless, this study clearly demonstrated that cohort-specific microbiota differences hamper finding a consensus biological interpretation between cross-sectional studies. This, combined with the complexity of individual disease pathogenesis, as well as the individual-specific differences in microbiota composition, may explain the inconsistency in observations between different studies concerning the identification of signature microbes for obesity, irritable bowel syndrome and other diseases.

Besides the biological drivers for cohort specific inconsistencies in identified microbial biomarkers, there are also technological factors. Although high-throughput sequencing of short, hypervariable segments of the 16S ribosomal RNA (rRNA) gene has transformed the methodological landscape describing microbial diversity within and across complex biomes, evidence is increasing that methodology rather than the biological variation is responsible for observed sample composition and distribution. Large meta-analyses would aid in elucidating whether the basis for these observed inconsistencies is biological, technical or maybe a combination of both. To facilitate these meta-analyses of microbiota studies we developed NG-Tax, a pipeline for 16S rRNA gene amplicon sequence analysis that was validated with different Mock Communities (MC). NG-Tax demonstrated high robustness against choice of region and other technical biases associated with 16S rRNA gene amplicon sequencing studies. The analysis of $\alpha$ - and $\beta$-diversity of these MC 
confirmed conclusions guided by biology rather than the methodological aspects. This pipeline was applied to biological samples to monitor the developing communities an in vitro gut model (TIM-2) fed either with a normal diet, or modified versions from which the carbohydrate (MPLC) or protein fraction was diluted (LPMC) for $72 \mathrm{~h}$. In combination with global metatranscriptomics and metabolomics this revealed that each diet produced distinct microbial communities and temporal patterns and ratios of metabolites. The microbiota in reactors fed diets containing normal carbohydrate levels were enriched in members of the genera Prevotella, Subdoligranulum, Blautia and Bifidobacterium, all associated with carbohydrate fermentation. In turn, the microbiota in the reactors fed the MPLC diet, containing ten-fold less carbohydrates, was enriched in the genus Bacteroides, which is associated with diets rich in protein and animal fat. This setup allows researchers to study the (trophic) interactions and task division within a community and how they are impacted by diet-related factors under controlled conditions, which may assist in defining causal links between specific diet-derived parameters microbial groups and their activities.

In conclusion, currently it seems that GI microbiota based biomarkers associated with metabolic impairments and anthropometric variables associated with the metabolic syndrome are cohort specific or possibly individual, which could partly be due to the use of incompatible analytical approaches. Nevertheless, there is growing evidence that human health is a collective property of the human body and its associated microbiome and thus requiring to study the interface of two very complex systems, i.e. on one side the extraordinary coding capacity, high inter-individuality and complex dynamics of the microbiome and on the other side the multifactorial individual nature of human disease. In light of these observations the manifestation of individual dynamics of the microbiota with the host when homeostasis is lost seems plausible and likely. 



\section{Appendices}

$\begin{array}{ll}\text { References } & 182\end{array}$

$\begin{array}{ll}\text { Co-author affiliations } & 198\end{array}$

Acknowledgements - Dankwoord 199

About the author $\quad 202$

List of publications $\quad 203$

Overview of completed training activities $\quad 204$ 


\section{References}

1. Flint HJ, Scott KP, Duncan SH, et al. Microbial degradation of complex carbohydrates in the gut. Gut Microbes 2012;3:289-306.

2. Mackowiak PA. Recycling metchnikoff: probiotics, the intestinal microbiome and the quest for long life. Front Public Health 2013;1:52.

3. Rajilic-Stojanovic M, Smidt H, de Vos WM. Diversity of the human gastrointestinal tract microbiota revisited. Environ Microbiol 2007;9:2125-36.

4. Marchesi JR, Ravel J. The vocabulary of microbiome research: a proposal. Microbiome 2015;3:31

5. Sender R, Fuchs S, Milo R. Are We Really Vastly Outnumbered? Revisiting the Ratio of Bacterial to Host Cells in Humans. Cell 2016;164:337-40.

6. Qin J, Li R, Raes J, et al. A human gut microbial gene catalogue established by metagenomic sequencing. Nature 2010;464:59-65.

7. Nielsen HB, Almeida M, Juncker AS, et al. Identification and assembly of genomes and genetic elements in complex metagenomic samples without using reference genomes. Nat Biotechnol 2014;32:822-8.

8. Li J, Jia H, Cai X, et al. An integrated catalog of reference genes in the human gut microbiome. Nat Biotechnol 2014;32:834-841.

9. Faith JJ, Guruge JL, Charbonneau M, et al. The long-term stability of the human gut microbiota Science 2013;341:1237439.

10. Aagaard K, Ma J, Antony KM, et al. The placenta harbors a unique microbiome. Sci Transl Med 2014;6:237ra65.

11. DiGiulio DB, Romero R, Amogan HP, et al. Microbial prevalence, diversity and abundance in amniotic fluid during preterm labor: a molecular and culture-based investigation. PLoS One 2008;3:e3056.

12. Jimenez E, Marin ML, Martin R, et al. Is meconium from healthy newborns actually sterile? Res Microbiol 2008;159:187-93.

13. Jimenez E, Fernandez L, Marin ML, et al. Isolation of commensal bacteria from umbilical cord blood of healthy neonates born by cesarean section. Curr Microbiol 2005;51:270-4.

14. Boggess KA, Watts DH, Hillier SL, et al. Bacteremia shortly after placental separation during cesarean delivery. Obstet Gynecol 1996;87:779-84.

15. Weiss S, Amir A, Hyde ER, et al. Tracking down the sources of experimental contamination in microbiome studies. Genome Biol 2014;15:564.

16. Salter SJ, Cox MJ, Turek EM, et al. Reagent and laboratory contamination can critically impact sequence-based microbiome analyses. BMC Biol 2014;12:87.

17. Lauder AP, Roche AM, Sherrill-Mix S, et al. Comparison of placenta samples with contamination controls does not provide evidence for a distinct placenta microbiota. Microbiome 2016;4.

18. Tamburini S, Shen N, Wu HC, et al. The microbiome in early life: implications for health outcomes. Nat Med 2016;22:713-22.

19. Jakobsson HE, Abrahamsson TR, Jenmalm MC, et al. Decreased gut microbiota diversity, delayed Bacteroidetes colonisation and reduced Th1 responses in infants delivered by caesarean section. Gut 2014;63:559-66.

20. Neu J, Rushing J. Cesarean versus vaginal delivery: long-term infant outcomes and the hygiene hypothesis. Clin Perinatol 2011;38:321-31.

21. Smith MI, Yatsunenko T, Manary MJ, et al. Gut microbiomes of Malawian twin pairs discordant for kwashiorkor. Science 2013;339:548-54.

22. Blanton LV, Charbonneau MR, Salih T, et al. Gut bacteria that prevent growth impairments transmitted by microbiota from malnourished children. Science 2016;351.

23. Subramanian S, Huq S, Yatsunenko T, et al. Persistent gut microbiota immaturity in malnourished Bangladeshi children. Nature 2014;510:417-21.

24. Planer JD, Peng Y, Kau AL, et al. Development of the gut microbiota and mucosal IgA responses in twins and gnotobiotic mice. Nature 2016;534:263-6.

25. Sela DA, Chapman J, Adeuya A, et al. The genome sequence of Bifidobacterium longum subsp. infantis reveals adaptations for milk utilization within the infant microbiome. Proc Natl Acad Sci U S A 2008;105:18964-9.

26. Marcobal A, Barboza M, Froehlich JW, et al. Consumption of human milk oligosaccharides by gutrelated microbes. J Agric Food Chem 2010;58:5334-40. 
27. Trosvik P, Stenseth NC, Rudi K. Convergent temporal dynamics of the human infant gut microbiota. ISME J 2010;4:151-8.

28. Favier CF, Vaughan EE, De Vos WM, et al. Molecular monitoring of succession of bacterial communities in human neonates. Appl Environ Microbiol 2002;68:219-26.

29. Koenig JE, Spor A, Scalfone N, et al. Succession of microbial consortia in the developing infant gut microbiome. Proc Natl Acad Sci U S A 2011;108 Suppl 1:4578-85.

30. Palmer C, Bik EM, DiGiulio DB, et al. Development of the human infant intestinal microbiota. PLoS Biol 2007;5:e177.

31. Costello EK, Stagaman K, Dethlefsen L, et al. The application of ecological theory toward an understanding of the human microbiome. Science 2012;336:1255-62.

32. Williams SC. Gnotobiotics. Proc Natl Acad Sci U S A 2014;111:1661.

33. Maynard CL, Elson CO, Hatton RD, et al. Reciprocal interactions of the intestinal microbiota and immune system. Nature 2012;489:231-41.

34. Zoetendal EG, Raes J, van den Bogert B, et al. The human small intestinal microbiota is driven by rapid uptake and conversion of simple carbohydrates. ISME J 2012.

35. Yatsunenko T, Rey FE, Manary MJ, et al. Human gut microbiome viewed across age and geography. Nature 2012;486:222-7

36. Turnbaugh PJ, Hamady M, Yatsunenko T, et al. A core gut microbiome in obese and lean twins. Nature 2009;457:480-4.

37. Goodrich JK, Waters JL, Poole AC, et al. Human genetics shape the gut microbiome. Cell 2014;159:789-99.

38. Richardson K, Norgate S. The equal environments assumption of classical twin studies may not hold. British Journal of Educational Psychology 2005;75:339-350.

39. Joseph J. Twin studies in psychiatry and psychology: science or pseudoscience? Psychiatr Q 2002;73:71-82.

40. Wacklin P, Tuimala J, Nikkila J, et al. Faecal Microbiota Composition in Adults Is Associated with the FUT2 Gene Determining the Secretor Status. Plos One 2014;9.

41. Petnicki-Ocwieja T, Hrncir T, Liu YJ, et al. Nod2 is required for the regulation of commensal microbiota in the intestine. Proceedings of the National Academy of Sciences of the United States of America 2009;106:15813-15818.

42. Rajilic-Stojanovic M, Heilig HG, Tims S, et al. Long-term monitoring of the human intestinal microbiota composition. Environ Microbiol 2012.

43. Caporaso JG, Lauber CL, Costello EK, et al. Moving pictures of the human microbiome. Genome Biol 2011;12:R50.

44. Relman DA. The human microbiome: ecosystem resilience and health. Nutr Rev 2012;70 Suppl 1:S2-9

45. Bodelier PL. Toward understanding, managing, and protecting microbial ecosystems. Front Microbiol 2011;2:80

46. Vital M, Howe AC, Tiedje JM. Revealing the bacterial butyrate synthesis pathways by analyzing (meta)genomic data. MBio 2014;5:e00889.

47. Walters WA, $\mathrm{Xu} \mathrm{Z}$, Knight R. Meta-analyses of human gut microbes associated with obesity and IBD FEBS Lett 2014;588:4223-33.

48. Turnbaugh PJ, Ley RE, Mahowald MA, et al. An obesity-associated gut microbiome with increased capacity for energy harvest. Nature 2006;444:1027-31.

49. Zhang X, Zhang D, Jia H, et al. The oral and gut microbiomes are perturbed in rheumatoid arthritis and partly normalized after treatment. Nat Med 2015;21:895-905.

50. Murri M, Leiva I, Gomez-Zumaquero JM, et al. Gut microbiota in children with type 1 diabetes differs from that in healthy children: a case-control study. BMC Med 2013;11:46.

51. Wacklin P, Kaukinen K, Tuovinen E, et al. The duodenal microbiota composition of adult celiac disease patients is associated with the clinical manifestation of the disease. Inflamm Bowel Dis 2013;19:934-41.

52. Simren M, Barbara G, Flint HJ, et al. Intestinal microbiota in functional bowel disorders: a Rome foundation report. Gut 2012.

53. Kostic AD, Xavier RJ, Gevers D. The microbiome in inflammatory bowel disease: current status and the future ahead. Gastroenterology 2014;146:1489-99.

54. Reddy BL, Saier MH. Autism and our intestinal microbiota. J Mol Microbiol Biotechnol 2015;25:51-5.

55. Scheperjans F, Aho V, Pereira PA, et al. Gut microbiota are related to Parkinson's disease and clinical phenotype. Mov Disord 2015;30:350-8. 
56. Venema K, van den Abbeele P. Experimental models of the gut microbiome. Best Pract Res Clin Gastroenterol 2013;27:115-26.

57. Van den Abbeele P, Venema K, Van de Wiele T, et al. Different human gut models reveal the distinct fermentation patterns of Arabinoxylan versus inulin. J Agric Food Chem 2013;61:9819-27.

58. van Nuenen MH, Venema K, van der Woude JC, et al. The metabolic activity of fecal microbiota from healthy individuals and patients with inflammatory bowel disease. Dig Dis Sci 2004;49:485-91.

59. Hugenholtz F ZJ, o'Toole PW, Marchesi J, Smidt H. Studying the mammalian intestinal microbiome using animal models. In: Yates MV NC, Miller RV, Pillai SD, ed. Manual of Environmental Microbiology. 4th edition ed. Washington D.C.: ASM Press, 2016:4.4.2-1 - 10.

60. Nguyen TL, Vieira-Silva S, Liston A, et al. How informative is the mouse for human gut microbiota research? Dis Model Mech 2015;8:1-16.

61. Davids M, Hugenholtz F, Martins dos Santos V, et al. Functional Profiling of Unfamiliar Microbial Communities Using a Validated De Novo Assembly Metatranscriptome Pipeline. PLoS One 2016;11:e0146423.

62. Ley RE, Backhed F, Turnbaugh P, et al. Obesity alters gut microbial ecology. Proc Natl Acad Sci U S A 2005;102:11070-5.

63. Goodman AL, Kallstrom G, Faith JJ, et al. Extensive personal human gut microbiota culture collections characterized and manipulated in gnotobiotic mice. Proc Natl Acad Sci U S A 2011;108:6252-7.

64. Turnbaugh PJ, Ridaura VK, Faith JJ, et al. The effect of diet on the human gut microbiome: a metagenomic analysis in humanized gnotobiotic mice. Sci Transl Med 2009;1:6ra14.

65. Everard A, Belzer C, Geurts L, et al. Cross-talk between Akkermansia muciniphila and intestinal epithelium controls diet-induced obesity. Proc Natl Acad Sci U S A 2013;110:9066-71.

66. Nell S, Suerbaum S, Josenhans C. The impact of the microbiota on the pathogenesis of IBD: lessons from mouse infection models. Nature Reviews Microbiology 2010;8:564-577.

67. Chung H, Pamp SJ, Hill JA, et al. Gut immune maturation depends on colonization with a host-specific microbiota. Cell 2012;149:1578-93.

68. Zhernakova A, Kurilshikov A, Bonder MJ, et al. Population-based metagenomics analysis reveals markers for gut microbiome composition and diversity. Science 2016;352:565-9.

69. Reijnders D, Goossens GH, Hermes GD, et al. Effects of Gut Microbiota Manipulation by Antibiotics on Host Metabolism in Obese Humans: A Randomized Double-Blind Placebo-Controlled Trial. Cell Metab 2016;24:63-74.

70. Marchesi JR, Adams DH, Fava F, et al. The gut microbiota and host health: a new clinical frontier. Gut 2015.

71. Jostins L, Ripke S, Weersma RK, et al. Host-microbe interactions have shaped the genetic architecture of inflammatory bowel disease. Nature 2012;491:119-124.

72. Paula H, Grover M, Halder SL, et al. Non-enteric infections, antibiotic use, and risk of development of functional gastrointestinal disorders. Neurogastroenterol Motil 2015;27:1580-6.

73. Barbara G, Feinle-Bisset C, Ghoshal UC, et al. The Intestinal Microenvironment and Functional Gastrointestinal Disorders. Gastroenterology 2016.

74. Nelson ML, Dinardo A, Hochberg J, et al. Brief communication: Mass spectroscopic characterization of tetracycline in the skeletal remains of an ancient population from Sudanese Nubia 350-550 CE. Am J Phys Anthropol 2010;143:151-4.

75. Bassett EJ, Keith MS, Armelagos GJ, et al. Tetracycline-labeled human bone from ancient Sudanese Nubia (A.D. 350). Science 1980;209:1532-4.

76. Aminov RI. A brief history of the antibiotic era: lessons learned and challenges for the future. Front Microbiol 2010;1:134.

77. Dethlefsen L, Huse S, Sogin ML, et al. The pervasive effects of an antibiotic on the human gut microbiota, as revealed by deep 16S rRNA sequencing. PLoS Biol 2008;6:e280.

78. Raymond F, Ouameur AA, Deraspe M, et al. The initial state of the human gut microbiome determines its reshaping by antibiotics. ISME J 2016;10:707-20.

79. Dethlefsen L, Relman DA. Incomplete recovery and individualized responses of the human distal gut microbiota to repeated antibiotic perturbation. Proc Natl Acad Sci U S A 2011;108 Suppl 1:4554-61.

80. Vrieze A, Out C, Fuentes S, et al. Impact of oral vancomycin on gut microbiota, bile acid metabolism, and insulin sensitivity. J Hepatol 2014;60:824-31.

81. Korpela K, Salonen A, Virta LJ, et al. Intestinal microbiome is related to lifetime antibiotic use in Finnish pre-school children. Nat Commun 2016;7:10410. 
82. Pitter G, Ludvigsson JF, Romor P, et al. Antibiotic exposure in the first year of life and later treated asthma, a population based birth cohort study of 143,000 children. Eur J Epidemiol 2016;31:85-94.

83. Penders J, Kummeling I, Thijs C. Infant antibiotic use and wheeze and asthma risk: a systematic review and meta-analysis. Eur Respir J 2011;38:295-302.

84. Jernberg C, Lofmark S, Edlund C, et al. Long-term ecological impacts of antibiotic administration on the human intestinal microbiota. ISME J 2007;1:56-66.

85. Penders J, Stobberingh EE, Savelkoul PH, et al. The human microbiome as a reservoir of antimicrobia resistance. Front Microbiol 2013;4:87.

86. Stecher B, Denzler R, Maier L, et al. Gut inflammation can boost horizontal gene transfer between pathogenic and commensal Enterobacteriaceae. Proceedings of the National Academy of Sciences of the United States of America 2012;109:1269-1274.

87. Karami N, Martner A, Enne VI, et al. Transfer of an ampicillin resistance gene between two Escherichia coli strains in the bowel microbiota of an infant treated with antibiotics. Journal of Antimicrobial Chemotherapy 2007;60:1142-1145.

88. van Nood E, Vrieze A, Nieuwdorp M, et al. Duodenal infusion of donor feces for recurrent Clostridium difficile. N Engl J Med 2013;368:407-15.

89. Rossen NG, Fuentes S, van der Spek MJ, et al. Findings From a Randomized Controlled Trial of Fecal Transplantation for Patients With Ulcerative Colitis. Gastroenterology 2015;149:110-118 e4.

90. Vrieze A, Van Nood E, Holleman F, et al. Transfer of intestinal microbiota from lean donors increases insulin sensitivity in individuals with metabolic syndrome. Gastroenterology 2012;143:913-916 e7.

91. Human Microbiome Consortium G. Structure, function and diversity of the healthy human microbiome. Nature 2012;486:207-14.

92. Arumugam M, Raes J, Pelletier E, et al. Enterotypes of the human gut microbiome. Nature 2011;473:174-80

93. Lahti L, Salojarvi J, Salonen A, et al. Tipping elements in the human intestinal ecosystem. Nat Commun 2014;5:4344.

94. Huse SM, Ye Y, Zhou Y, et al. A core human microbiome as viewed through 16S rRNA sequence clusters. PLoS One 2012;7:e34242.

95. Salonen A, Salojarvi J, Lahti L, et al. The adult intestinal core microbiota is determined by analysis depth and health status. Clin Microbiol Infect 2012;18 Suppl 4:16-20.

96. Tap J, Mondot S, Levenez F, et al. Towards the human intestinal microbiota phylogenetic core. Environ Microbiol 2009;11:2574-84.

97. Zoetendal EG, Rajilic-Stojanovic M, de Vos WM. High-throughput diversity and functionality analysis of the gastrointestinal tract microbiota. Gut 2008;57:1605-15.

98. Flint HJ, Bayer EA, Rincon MT, et al. Polysaccharide utilization by gut bacteria: potential for new insights from genomic analysis. Nat Rev Microbiol 2008;6:121-31.

99. Van Laere KM, Hartemink R, Bosveld M, et al. Fermentation of plant cell wall derived polysaccharides and their corresponding oligosaccharides by intestinal bacteria. J Agric Food Chem 2000;48:1644-52.

100. Zoetendal EG, Akkermans AD, De Vos WM. Temperature gradient gel electrophoresis analysis of $16 \mathrm{~S}$ rRNA from human fecal samples reveals stable and host-specific communities of active bacteria. Appl Environ Microbiol 1998;64:3854-9.

101. Claesson MJ, Cusack S, O'Sullivan O, et al. Composition, variability, and temporal stability of the intestinal microbiota of the elderly. Proc Natl Acad Sci U S A 2011;108 Suppl 1:4586-91.

102. Claesson MJ, Jeffery IB, Conde S, et al. Gut microbiota composition correlates with diet and health in the elderly. Nature 2012;488:178-84.

103. Backhed F, Ding H, Wang T, et al. The gut microbiota as an environmental factor that regulates fat storage. Proc Natl Acad Sci U S A 2004;101:15718-23.

104. Ley RE, Turnbaugh PJ, Klein S, et al. Microbial ecology: human gut microbes associated with obesity. Nature 2006;444:1022-3.

105. Raes J, Bork P. Molecular eco-systems biology: towards an understanding of community function. Nat Rev Microbiol 2008;6:693-9.

106. Ferrer M, Ruiz A, Lanza F, et al. Microbiota from the distal guts of lean and obese adolescents exhibit partial functional redundancy besides clear differences in community structure. Environ Microbiol 2012.

107. Carrigg C, Rice O, Kavanagh S, et al. DNA extraction method affects microbial community profiles from soils and sediment. Appl Microbiol Biotechnol 2007;77:955-64. 
108. Yuan S, Cohen DB, Ravel J, et al. Evaluation of methods for the extraction and purification of DNA from the human microbiome. PLoS One 2012; 7:e33865.

109. Salonen A, Nikkila J, Jalanka-Tuovinen J, et al. Comparative analysis of fecal DNA extraction methods with phylogenetic microarray: effective recovery of bacterial and archaeal DNA using mechanical cell lysis. J Microbiol Methods 2010;81:127-34.

110. Zoetendal EG, Heilig HG, Klaassens ES, et al. Isolation of DNA from bacterial samples of the human gastrointestinal tract. Nat Protoc 2006;1:870-3.

111. DeSantis TZ, Hugenholtz P, Larsen N, et al. Greengenes, a chimera-checked 16S rRNA gene database and workbench compatible with ARB. Appl Environ Microbiol 2006;72:5069-72.

112. McDonald D, Price MN, Goodrich J, et al. An improved Greengenes taxonomy with explicit ranks for ecological and evolutionary analyses of bacteria and archaea. ISME J 2012;6:610-8.

113. Pruesse E, Quast C, Knittel K, et al. SILVA: a comprehensive online resource for quality checked and aligned ribosomal RNA sequence data compatible with ARB. Nucleic Acids Res 2007;35:7188-96.

114. Cole JR, Wang Q, Cardenas E, et al. The Ribosomal Database Project: improved alignments and new tools for rRNA analysis. Nucleic Acids Res 2009;37:D141-5.

115. Fox GE, Wisotzkey JD, Jurtshuk P, Jr. How close is close: 16S rRNA sequence identity may not be sufficient to guarantee species identity. Int J Syst Bacteriol 1992;42:166-70.

116. Stackebrandt E, Frederiksen W, Garrity GM, et al. Report of the ad hoc committee for the re-evaluation of the species definition in bacteriology. Int J Syst Evol Microbiol 2002;52:1043-7.

117. Stackenbrandt E EJ. Taxonomic parameters revisited: tarnished gold standards Microbiology Today. Volume 33, 2006:152-155.

118. Gevers D, Cohan FM, Lawrence JG, et al. Opinion: Re-evaluating prokaryotic species. Nat Rev Microbiol 2005;3:733-9.

119. Ley RE. Obesity and the human microbiome. Curr Opin Gastroenterol 2010;26:5-11.

120. Muyzer G, de Waal EC, Uitterlinden AG. Profiling of complex microbial populations by denaturing gradient gel electrophoresis analysis of polymerase chain reaction-amplified genes coding for $16 \mathrm{~S}$ rRNA. Appl Environ Microbiol 1993;59:695-700.

121. Eckburg PB, Bik EM, Bernstein CN, et al. Diversity of the human intestinal microbial flora. Science 2005;308:1635-8

122. Wang Q, Garrity GM, Tiedje JM, et al. Naive Bayesian classifier for rapid assignment of rRNA sequences into the new bacterial taxonomy. Appl Environ Microbiol 2007;73:5261-7.

123. Amann RI, Ludwig W, Schleifer KH. Phylogenetic identification and in situ detection of individual microbial cells without cultivation. Microbiol Rev 1995;59:143-69.

124. Hamady M, Walker JJ, Harris JK, et al. Error-correcting barcoded primers for pyrosequencing hundreds of samples in multiplex. Nat Methods 2008;5:235-7.

125. Liu Z, DeSantis TZ, Andersen GL, et al. Accurate taxonomy assignments from 16S rRNA sequences produced by highly parallel pyrosequencers. Nucleic Acids Res 2008;36:e120.

126. Kuczynski J, Lauber CL, Walters WA, et al. Experimental and analytical tools for studying the human microbiome. Nat Rev Genet 2012;13:47-58.

127. Claesson MJ, Wang Q, O'Sullivan O, et al. Comparison of two next-generation sequencing technologies for resolving highly complex microbiota composition using tandem variable 16S rRNA gene regions. Nucleic Acids Res 2010;38:e200.

128. Caporaso JG, Lauber CL, Walters WA, et al. Global patterns of 16S rRNA diversity at a depth of millions of sequences per sample. Proc Natl Acad Sci U S A 2011;108 Suppl 1:4516-22.

129. Caporaso JG, Lauber CL, Walters WA, et al. Ultra-high-throughput microbial community analysis on the Illumina HiSeq and MiSeq platforms. ISME J 2012;6:1621-4.

130. Werner JJ, Zhou D, Caporaso JG, et al. Comparison of Illumina paired-end and single-direction sequencing for microbial 16S rRNA gene amplicon surveys. ISME J 2012;6:1273-6.

131. Zhou HW, Li DF, Tam NF, et al. BIPES, a cost-effective high-throughput method for assessing microbial diversity. ISME J 2011;5:741-9.

132. Bartram AK, Lynch MD, Stearns JC, et al. Generation of multimillion-sequence 16S rRNA gene libraries from complex microbial communities by assembling paired-end illumina reads. Appl Environ Microbiol 2011;77:3846-52.

133. Kuczynski J, Stombaugh J, Walters WA, et al. Using QIIME to analyze 16S rRNA gene sequences from microbial communities. Curr Protoc Bioinformatics 2011; Chapter 10:Unit 107. 
134. Schloss PD, Westcott SL, Ryabin T, et al. Introducing mothur: open-source, platform-independent, community-supported software for describing and comparing microbial communities. Appl Environ Microbiol 2009;75:7537-41.

135. Reeder J, Knight R. The 'rare biosphere': a reality check. Nat Methods 2009;6:636-7.

136. Reeder J, Knight R. Rapidly denoising pyrosequencing amplicon reads by exploiting rank-abundance distributions. Nat Methods 2010;7:668-9.

137. Kunin V, Engelbrektson A, Ochman H, et al. Wrinkles in the rare biosphere: pyrosequencing errors can lead to artificial inflation of diversity estimates. Environ Microbiol 2010;12:118-23.

138. Quince C, Lanzen A, Davenport RJ, et al. Removing noise from pyrosequenced amplicons. BMC Bioinformatics 2011;12:38.

139. Stackebrandt E, Goebel BM. A place for DNA-DNA reassociation and 16S ribosomal-RNA sequenceanalysis in the present species definition in bacteriology. Int. J. Syst. Bacteriol. 1994;44:846849.

140. Sun Y, Cai Y, Huse SM, et al. A large-scale benchmark study of existing algorithms for taxonomyindependent microbial community analysis. Brief Bioinform 2011.

141. Wang L, Si Y, Dedow LK, et al. A low-cost library construction protocol and data analysis pipeline for Illumina-based strand-specific multiplex RNA-seq. PLoS One 2011;6:e26426.

142. Huse SM, Dethlefsen L, Huber JA, et al. Exploring microbial diversity and taxonomy using SSU rRNA hypervariable tag sequencing. PLoS Genet 2008;4:e1000255.

143. Liu Z, Lozupone C, Hamady M, et al. Short pyrosequencing reads suffice for accurate microbial community analysis. Nucleic Acids Res 2007;35:e120.

144. Soergel DA, Dey N, Knight R, et al. Selection of primers for optimal taxonomic classification of environmental 16S rRNA gene sequences. ISME J 2012

145. Kim M, Morrison M, Yu Z. Evaluation of different partial 16S rRNA gene sequence regions for phylogenetic analysis of microbiomes. J Microbiol Methods 2011;84:81-7.

146. Hayashi H, Sakamoto M, Benno Y. Evaluation of three different forward primers by terminal restriction fragment length polymorphism analysis for determination of fecal bifidobacterium spp. in healthy subjects. Microbiol Immunol 2004;48:1-6.

147. Bergmann GT, Bates ST, Eilers KG, et al. The under-recognized dominance of Verrucomicrobia in soil bacterial communities. Soil Biol Biochem 2011;43:1450-1455.

148. Walters WA, Caporaso JG, Lauber CL, et al. PrimerProspector: de novo design and taxonomic analysis of barcoded polymerase chain reaction primers. Bioinformatics 2011;27:1159-61.

149. Wu GD, Lewis JD, Hoffmann C, et al. Sampling and pyrosequencing methods for characterizing bacterial communities in the human gut using 16S sequence tags. BMC Microbiol 2010;10:206.

150. Human Microbiome Project Consortium G. A framework for human microbiome research. Nature 2012;486:215-21

151. Jumpstart Consortium Human Microbiome Project Data Generation Working G. Evaluation of 16S rDNA-based community profiling for human microbiome research. PLoS One 2012;7:e39315.

152. Huse SM, Huber JA, Morrison HG, et al. Accuracy and quality of massively parallel DNA pyrosequencing. Genome Biol 2007;8:R143.

153. Schloss PD, Gevers D, Westcott SL. Reducing the Effects of PCR Amplification and Sequencing Artifacts on 16S rRNA-Based Studies. Plos One 2011;6.

154. Andersson AF, Lindberg M, Jakobsson H, et al. Comparative analysis of human gut microbiota by barcoded pyrosequencing. PLoS One 2008;3:e2836.

155. Claesson MJ, O'Sullivan O, Wang Q, et al. Comparative analysis of pyrosequencing and a phylogenetic microarray for exploring microbial community structures in the human distal intestine. PLoS One 2009;4:e6669.

156. van den Bogert B, de Vos WM, Zoetendal EG, et al. Microarray analysis and barcoded pyrosequencing provide consistent microbial profiles depending on the source of human intestinal samples. Appl Environ Microbiol 2011;77:2071-80.

157. Zupancic ML, Cantarel BL, Liu Z, et al. Analysis of the gut microbiota in the old order amish and its relation to the metabolic syndrome. PLoS One 2012; 7:e43052.

158. Candela M, Consolandi C, Severgnini M, et al. High taxonomic level fingerprint of the human intestinal microbiota by ligase detection reaction--universal array approach. BMC Microbiol 2010;10:116 
159. Rajilic-Stojanovic M, Heilig HG, Molenaar D, et al. Development and application of the human intestinal tract chip, a phylogenetic microarray: analysis of universally conserved phylotypes in the abundant microbiota of young and elderly adults. Environ Microbiol 2009;11:1736-51.

160. Brodie EL, Desantis TZ, Joyner DC, et al. Application of a high-density oligonucleotide microarray approach to study bacterial population dynamics during uranium reduction and reoxidation. Appl Environ Microbiol 2006;72:6288-98.

161. Paliy O, Kenche H, Abernathy F, et al. High-throughput quantitative analysis of the human intestinal microbiota with a phylogenetic microarray. Appl Environ Microbiol 2009;75:3572-9.

162. Paliy O, Agans R. Application of phylogenetic microarrays to interrogation of human microbiota. FEMS Microbiol Ecol 2012;79:2-11.

163. Nikkila J, de Vos WM. Advanced approaches to characterize the human intestinal microbiota by computational meta-analysis. J Clin Gastroenterol 2010;44 Suppl 1:S2-5.

164. Turnbaugh PJ, Backhed F, Fulton L, et al. Diet-induced obesity is linked to marked but reversible alterations in the mouse distal gut microbiome. Cell Host Microbe 2008;3:213-23.

165. Hildebrandt MA, Hoffmann C, Sherrill-Mix SA, et al. High-fat diet determines the composition of the murine gut microbiome independently of obesity. Gastroenterology 2009;137:1716-24 e1-2.

166. de Wit N, Derrien M, Bosch-Vermeulen H, et al. Saturated fat stimulates obesity and hepatic steatosis and affects gut microbiota composition by an enhanced overflow of dietary fat to the distal intestine. Am J Physiol Gastrointest Liver Physiol 2012;303:G589-99.

167. Murphy EF, Cotter PD, Healy S, et al. Composition and energy harvesting capacity of the gut microbiota: relationship to diet, obesity and time in mouse models. Gut 2010;59:1635-42.

168. Ravussin Y, Koren O, Spor A, et al. Responses of gut microbiota to diet composition and weight loss in lean and obese mice. Obesity (Silver Spring) 2012;20:738-47.

169. Santacruz A, Collado MC, Garcia-Valdes L, et al. Gut microbiota composition is associated with body weight, weight gain and biochemical parameters in pregnant women. Br J Nutr 2010;104:83-92.

170. Duncan SH, Lobley GE, Holtrop G, et al. Human colonic microbiota associated with diet, obesity and weight loss. Int J Obes (Lond) 2008;32:1720-4.

171. Zhang H, DiBaise JK, Zuccolo A, et al. Human gut microbiota in obesity and after gastric bypass. Proc Natl Acad Sci U S A 2009;106:2365-70.

172. Collado MC, Isolauri E, Laitinen K, et al. Distinct composition of gut microbiota during pregnancy in overweight and normal-weight women. Am J Clin Nutr 2008;88:894-9.

173. Schwiertz A, Taras D, Schafer K, et al. Microbiota and SCFA in Lean and Overweight Healthy Subjects. Obesity 2010;18:190-195.

174. Verdam FJ, Fuentes S, de Jonge C, et al. Human intestinal microbiota composition is associated with local and systemic inflammation in obesity. Obesity (Silver Spring) 2013.

175. Faith JJ, McNulty NP, Rey FE, et al. Predicting a human gut microbiota's response to diet in gnotobiotic mice. Science 2011;333:101-4.

176. Romero-Corral A, Somers VK, Sierra-Johnson J, et al. Accuracy of body mass index in diagnosing obesity in the adult general population. Int J Obes (Lond) 2008;32:959-66.

177. Bluher M. The distinction of metabolically 'healthy' from 'unhealthy' obese individuals. Curr Opin Lipidol 2010;21:38-43.

178. Furet JP, Kong LC, Tap J, et al. Differential adaptation of human gut microbiota to bariatric surgeryinduced weight loss: links with metabolic and low-grade inflammation markers. Diabetes 2010;59:3049-57.

179. Jumpertz R, Le DS, Turnbaugh PJ, et al. Energy-balance studies reveal associations between gut microbes, caloric load, and nutrient absorption in humans. Am J Clin Nutr 2011;94:58-65.

180. Salonen A, de Vos WM. Impact of diet on human intestinal microbiota and health. Annu Rev Food Sci Technol 2014;5:239-62.

181. Munukka E, Wiklund P, Pekkala S, et al. Women with and without metabolic disorder differ in their gut microbiota composition. Obesity (Silver Spring) 2012;20:1082-7.

182. Larsen N, Vogensen FK, van den Berg FW, et al. Gut microbiota in human adults with type 2 diabetes differs from non-diabetic adults. PLoS One 2010;5:e9085.

183. Greiner T, Backhed F. Effects of the gut microbiota on obesity and glucose homeostasis. Trends Endocrinol Metab 2011;22:117-23.

184. Tims S, Derom C, Jonkers DM, et al. Microbiota conservation and BMI signatures in adult monozygotic twins. ISME J 2012 
185. Turnbaugh PJ, Quince C, Faith JJ, et al. Organismal, genetic, and transcriptional variation in the deeply sequenced gut microbiomes of identical twins. Proc Natl Acad Sci U S A 2010;107:7503-8.

186. Zoetendal EG AA, Akkermans-Van Vliet WM, de Visser A, de Vos WM. The host genotype affects the bacterial community in the human gastrointestinal tract. . Microbial Ecol Health Dis 2001;13:12934.

187. Langille MG, Zaneveld J, Caporaso JG, et al. Predictive functional profiling of microbial communities using 16S rRNA marker gene sequences. Nat Biotechnol 2013;31:814-21.

188. Handelsman J. Metagenomics: application of genomics to uncultured microorganisms. Microbiol Mol Biol Rev 2004;68:669-85

189. Tasse L, Bercovici J, Pizzut-Serin S, et al. Functional metagenomics to mine the human gut microbiome for dietary fiber catabolic enzymes. Genome Res 2010;20:1605-12.

190. Tyson GW, Chapman J, Hugenholtz P, et al. Community structure and metabolism through reconstruction of microbial genomes from the environment. Nature 2004;428:37-43.

191. Gill SR, Pop M, Deboy RT, et al. Metagenomic analysis of the human distal gut microbiome. Science 2006;312:1355-9.

192. Kurokawa K, Itoh T, Kuwahara T, et al. Comparative metagenomics revealed commonly enriched gene sets in human gut microbiomes. DNA Research 2007;14:169-181.

193. Nelson KE, Weinstock GM, Highlander SK, et al. A catalog of reference genomes from the human microbiome. Science 2010;328:994-9.

194. Greenblum S, Turnbaugh PJ, Borenstein E. Metagenomic systems biology of the human gut microbiome reveals topological shifts associated with obesity and inflammatory bowel disease. Proc Natl Acad Sci U S A 2012;109:594-9.

195. Qin J, Li Y, Cai Z, et al. A metagenome-wide association study of gut microbiota in type 2 diabetes. Nature 2012;490:55-60.

196. Le Chatelier E, Nielsen T, Qin J, et al. Richness of human gut microbiome correlates with metabolic markers. Nature 2013;500:541-6.

197. Cotillard A, Kennedy SP, Kong LC, et al. Dietary intervention impact on gut microbial gene richness Nature 2013;500:585-8.

198. Wu GD, Chen J, Hoffmann C, et al. Linking long-term dietary patterns with gut microbial enterotypes. Science 2011;334:105-8.

199. Gosalbes MJ, Durban A, Pignatelli M, et al. Metatranscriptomic approach to analyze the functional human gut microbiota. PLoS One 2011;6:e17447.

200. Mahowald MA, Rey FE, Seedorf H, et al. Characterizing a model human gut microbiota composed of members of its two dominant bacterial phyla. Proc Natl Acad Sci U S A 2009;106:5859-64.

201. Booijink CC, Boekhorst J, Zoetendal EG, et al. Metatranscriptome analysis of the human fecal microbiota reveals subject-specific expression profiles, with genes encoding proteins involved in carbohydrate metabolism being dominantly expressed. Appl Environ Microbiol 2010;76:5533-40.

202. Simon C, Daniel R. Metagenomic analyses: past and future trends. Appl Environ Microbiol 2011;77:1153-61.

203. Moran MA, Satinsky B, Gifford SM, et al. Sizing up metatranscriptomics. ISME J 2012.

204. Leimena MM, Ramiro-Garcia J, Davids M, et al. A comprehensive metatranscriptome analysis pipeline and its validation using human small intestine microbiota datasets. BMC Genomics 2013;14:530.

205. Wilmes P, Bond PL. Metaproteomics: studying functional gene expression in microbial ecosystems. Trends Microbiol 2006;14:92-7.

206. Klaassens ES, de Vos WM, Vaughan EE. Metaproteomics approach to study the functionality of the microbiota in the human infant gastrointestinal tract. Appl Environ Microbiol 2007;73:1388-92.

207. Schneider T, Riedel K. Environmental proteomics: analysis of structure and function of microbial communities. Proteomics 2010;10:785-98.

208. Marcotte EM. How do shotgun proteomics algorithms identify proteins? Nature Biotechnology 2007; $25: 755-757$

209. Verberkmoes NC, Russell AL, Shah M, et al. Shotgun metaproteomics of the human distal gut microbiota. ISME J 2009;3:179-89.

210. Rooijers K, Kolmeder C, Juste C, et al. An iterative workflow for mining the human intestinal metaproteome. BMC Genomics 2011;12:6.

211. Kolmeder CA, de Been M, Nikkila J, et al. Comparative metaproteomics and diversity analysis of human intestinal microbiota testifies for its temporal stability and expression of core functions. PLoS One 2012;7:e29913. 
212. Derrien M, Collado MC, Ben-Amor K, et al. The Mucin degrader Akkermansia muciniphila is an abundant resident of the human intestinal tract. Appl Environ Microbiol 2008;74:1646-8.

213. Kolmeder CA, de Vos WM. Metaproteomics of our microbiome - Developing insight in function and activity in man and model systems. J Proteomics 2013.

214. Islam KB, Fukiya S, Hagio M, et al. Bile acid is a host factor that regulates the composition of the cecal microbiota in rats. Gastroenterology 2011;141:1773-81.

215. Turnbaugh PJ, Gordon JI. An invitation to the marriage of metagenomics and metabolomics. Cell 2008;134:708-13.

216. Nicholson JK, Lindon JC. Systems biology: Metabonomics. Nature 2008;455:1054-6.

217. Sahoo K, Sahoo B, Choudhury AK, et al. Childhood obesity: causes and consequences. J Family Med Prim Care 2015;4:187-92.

218. Joyce SA, MacSharry J, Casey PG, et al. Regulation of host weight gain and lipid metabolism by bacterial bile acid modification in the gut. Proc Natl Acad Sci U S A 2014;111:7421-6.

219. Cani PD, Bibiloni R, Knauf C, et al. Changes in gut microbiota control metabolic endotoxemia-induced inflammation in high-fat diet-induced obesity and diabetes in mice. Diabetes 2008;57:1470-81.

220. Cani PD, Amar J, Iglesias MA, et al. Metabolic endotoxemia initiates obesity and insulin resistance. Diabetes 2007;56:1761-72.

221. Zuo HJ, Xie ZM, Zhang WW, et al. Gut bacteria alteration in obese people and its relationship with gene polymorphism. World J Gastroenterol 2011;17:1076-81.

222. Xu P, Li M, Zhang J, et al. Correlation of intestinal microbiota with overweight and obesity in Kazakh school children. BMC Microbiol 2012;12:283.

223. Armougom F, Henry M, Vialettes B, et al. Monitoring bacterial community of human gut microbiota reveals an increase in Lactobacillus in obese patients and Methanogens in anorexic patients. PLoS One 2009;4:e7125.

224. Mai V, McCrary QM, Sinha R, et al. Associations between dietary habits and body mass index with gut microbiota composition and fecal water genotoxicity: an observational study in African American and Caucasian American volunteers. Nutr J 2009;8:49.

225. Hu HJ, Park SG, Jang HB, et al. Obesity Alters the Microbial Community Profile in Korean Adolescents. PLoS One 2015;10:e0134333.

226. Fernandes J, Su W, Rahat-Rozenbloom S, et al. Adiposity, gut microbiota and faecal short chain fatty acids are linked in adult humans. Nutr Diabetes 2014;4:e121.

227. Scheepers LE, Penders J, Mbakwa CA, et al. The intestinal microbiota composition and weight development in children: the KOALA Birth Cohort Study. Int J Obes (Lond) 2015;39:16-25.

228. Kalliomaki M, Collado MC, Salminen S, et al. Early differences in fecal microbiota composition in children may predict overweight. Am J Clin Nutr 2008;87:534-8.

229. Karlsson CL, Onnerfalt J, Xu J, et al. The microbiota of the gut in preschool children with normal and excessive body weight. Obesity (Silver Spring) 2012;20:2257-61.

230. Balamurugan R, George G, Kabeerdoss J, et al. Quantitative differences in intestinal Faecalibacterium prausnitzii in obese Indian children. Br J Nutr 2010;103:335-8.

231. Mbakwa CA, Penders J, Savelkoul PH, et al. Gut colonization with methanobrevibacter smithii is associated with childhood weight development. Obesity (Silver Spring) 2015;23:2508-16.

232. Hermes GD, Zoetendal EG, Smidt H. Molecular ecological tools to decipher the role of our microbial mass in obesity. Benef Microbes 2015;6:61-81.

233. Kummeling I, Thijs C, Penders J, et al. Etiology of atopy in infancy: the KOALA Birth Cohort Study. Pediatr Allergy Immunol 2005;16:679-84.

234. Bastiaanssen JM, de Bie RA, Bastiaenen $\mathrm{CH}$, et al. Etiology and prognosis of pregnancy-related pelvic girdle pain; design of a longitudinal study. BMC Public Health 2005;5:1.

235. Jalanka-Tuovinen J, Salonen A, Nikkila J, et al. Intestinal microbiota in healthy adults: temporal analysis reveals individual and common core and relation to intestinal symptoms. PLoS One 2011;6:e23035.

236. Jari Oksanen F KR, Legendre P, Minchin PR, O’Hara R, Simpson GL. Vegan: Community ecology package. 2013.

237. Fredriks AM, van Buuren S, Wit JM, et al. Body index measurements in 1996-7 compared with 1980. Arch Dis Child 2000;82:107-12.

238. Ogden CL, Flegal KM. Changes in terminology for childhood overweight and obesity. Natl Health Stat Report 2010:1-5. 
239. Šmilauer P LJ. Multivariate analysis of ecological data using canoco 5: Cambridge University Press, 2014.

240. Zou H HT. Regularization and variable selection via the elastic net. Journal of the Royal Statistical Society: Series B (Statistical Methodology). 2005:301-320.

241. Livina VN KF, Lenton TM. Potential analysis reveals changing number of climate states during the last 60 kyr. Climate of the Past 2010:77-82.

242. Dakos V, Carpenter SR, Brock WA, et al. Methods for detecting early warnings of critical transitions in time series illustrated using simulated ecological data. PLoS One 2012;7:e41010.

243. Hollister EB, Riehle K, Luna RA, et al. Structure and function of the healthy pre-adolescent pediatric gut microbiome. Microbiome 2015;3:36.

244. Bervoets L, Van Hoorenbeeck K, Kortleven I, et al. Differences in gut microbiota composition between obese and lean children: a cross-sectional study. Gut Pathog 2013;5:10

245. Kasai C, Sugimoto K, Moritani I, et al. Comparison of the gut microbiota composition between obese and non-obese individuals in a Japanese population, as analyzed by terminal restriction fragment length polymorphism and next-generation sequencing. BMC Gastroenterol 2015;15:100.

246. Lozupone CA, Stombaugh JI, Gordon JI, et al. Diversity, stability and resilience of the human gut microbiota. Nature 2012;489:220-30.

247. Yasir M, Angelakis E, Bibi F, et al. Comparison of the gut microbiota of people in France and Saudi Arabia. Nutr Diabetes 2015;5:e153.

248. Everard A, Lazarevic V, Derrien M, et al. Responses of gut microbiota and glucose and lipid metabolism to prebiotics in genetic obese and diet-induced leptin-resistant mice. Diabetes 2011;60:2775-86

249. Remely M, Hippe B, Geretschlaeger I, et al. Increased gut microbiota diversity and abundance of Faecalibacterium prausnitzii and Akkermansia after fasting: a pilot study. Wien Klin Wochenschr 2015;127:394-8.

250. Wolin MJ, Miller TL, Collins MD, et al. Formate-dependent growth and homoacetogenic fermentation by a bacterium from human feces: description of Bryantella formatexigens gen. nov., sp. nov. Appl Environ Microbiol 2003;69:6321-6.

251. Frost G, Sleeth ML, Sahuri-Arisoylu M, et al. The short-chain fatty acid acetate reduces appetite via a central homeostatic mechanism. Nat Commun 2014;5:3611.

252. Wang M, Radlowski EC, Monaco MH, et al. Mode of delivery and early nutrition modulate microbia colonization and fermentation products in neonatal piglets. J Nutr 2013;143:795-803.

253. Lauber CL, Zhou N, Gordon JI, et al. Effect of storage conditions on the assessment of bacterial community structure in soil and human-associated samples. FEMS Microbiol Lett 2010;307:80-6.

254. Tedjo DI, Jonkers DM, Savelkoul PH, et al. The effect of sampling and storage on the fecal microbiota composition in healthy and diseased subjects. PLoS One 2015;10:e0126685.

255. Vael C, Verhulst SL, Nelen V, et al. Intestinal microflora and body mass index during the first three years of life: an observational study. Gut Pathog 2011;3:8.

256. Khan MT, Nieuwdorp M, Backhed F. Microbial modulation of insulin sensitivity. Cell Metab 2014;20:753-60.

257. Greenhill C. Obesity: Gut microbiota, host genetics and diet interact to affect the risk of developing obesity and the metabolic syndrome. Nat Rev Endocrinol 2015.

258. Vrieze A, de Groot PF, Kootte RS, et al. Fecal transplant: a safe and sustainable clinical therapy for restoring intestinal microbial balance in human disease? Best Pract Res Clin Gastroenterol 2013;27:127-37.

259. Hartstra AV, Bouter KE, Backhed F, et al. Insights into the role of the microbiome in obesity and type 2 diabetes. Diabetes Care 2015;38:159-65

260. Cox LM, Blaser MJ. Pathways in microbe-induced obesity. Cell Metab 2013;17:883-94.

261. Jones ML, Martoni CJ, Ganopolsky JG, et al. The human microbiome and bile acid metabolism: dysbiosis, dysmetabolism, disease and intervention. Expert Opin Biol Ther 2014;14:467-82.

262. Canfora EE, Jocken JW, Blaak EE. Short-chain fatty acids in control of body weight and insulin sensitivity. Nat Rev Endocrinol 2015;11:577-91.

263. Jess T. Microbiota, antibiotics, and obesity. N Engl J Med 2014;371:2526-8

264. Cox LM, Blaser MJ. Antibiotics in early life and obesity. Nat Rev Endocrinol 2015;11:182-90.

265. Chou CJ, Membrez M, Blancher F. Gut decontamination with norfloxacin and ampicillin enhances insulin sensitivity in mice. Nestle Nutr Workshop Ser Pediatr Program 2008;62:127-37; discussion 137-40. 
266. Hwang I, Park YJ, Kim YR, et al. Alteration of gut microbiota by vancomycin and bacitracin improves insulin resistance via glucagon-like peptide 1 in diet-induced obesity. FASEB J 2015;29:2397-411.

267. Membrez M, Blancher F, Jaquet M, et al. Gut microbiota modulation with norfloxacin and ampicillin enhances glucose tolerance in mice. Faseb Journal 2008;22:2416-2426.

268. Murphy EF, Cotter PD, Hogan A, et al. Divergent metabolic outcomes arising from targeted manipulation of the gut microbiota in diet-induced obesity. Gut 2013;62:220-6.

269. Hulzebos CV, Renfurm L, Bandsma RH, et al. Measurement of parameters of cholic acid kinetics in plasma using a microscale stable isotope dilution technique: application to rodents and humans. J Lipid Res 2001;42:1923-9.

270. DeFronzo RA, Tobin JD, Andres R. Glucose clamp technique: a method for quantifying insulin secretion and resistance. Am J Physiol 1979;237:E214-23.

271. Steele R. Influences of glucose loading and of injected insulin on hepatic glucose output. Ann N Y Acad Sci 1959;82:420-30

272. Kotronen A, Seppala-Lindroos A, Bergholm R, et al. Tissue specificity of insulin resistance in humans: fat in the liver rather than muscle is associated with features of the metabolic syndrome. Diabetologia 2008;51:130-8.

273. Brehm A, Krssak M, Schmid AI, et al. Increased lipid availability impairs insulin-stimulated ATP synthesis in human skeletal muscle. Diabetes 2006;55:136-40.

274. Most J, Timmers S, Warnke I, et al. Combined epigallocatechin-3-gallate and resveratrol supplementation for $12 \mathrm{wk}$ increases mitochondrial capacity and fat oxidation, but not insulin sensitivity, in obese humans: a randomized controlled trial. Am J Clin Nutr 2016.

275. Schoffelen PF, Westerterp KR, Saris WH, et al. A dual-respiration chamber system with automated calibration. J Appl Physiol (1985) 1997;83:2064-72.

276. Weir JB. New methods for calculating metabolic rate with special reference to protein metabolism. J Physiol 1949;109:1-9.

277. Frayn KN. Calculation of substrate oxidation rates in vivo from gaseous exchange. J Appl Physiol Respir Environ Exerc Physiol 1983;55:628-34.

278. Jans A, Konings E, Goossens GH, et al. PUFAs acutely affect triacylglycerol-derived skeletal muscle fatty acid uptake and increase postprandial insulin sensitivity. Am J Clin Nutr 2012;95:825-36.

279. van Wijck K, Verlinden TJ, van Eijk HM, et al. Novel multi-sugar assay for site-specific gastrointestinal permeability analysis: a randomized controlled crossover trial. Clin Nutr 2013;32:24551.

280. Kersten S, Lichtenstein L, Steenbergen E, et al. Caloric restriction and exercise increase plasma ANGPTL4 levels in humans via elevated free fatty acids. Arterioscler Thromb Vasc Biol 2009;29:96974.

281. Schols AM, Buurman WA, Staal van den Brekel AJ, et al. Evidence for a relation between metabolic derangements and increased levels of inflammatory mediators in a subgroup of patients with chronic obstructive pulmonary disease. Thorax 1996;51:819-24.

282. Vilsboll T, Krarup T, Sonne J, et al. Incretin secretion in relation to meal size and body weight in healthy subjects and people with type 1 and type 2 diabetes mellitus. J Clin Endocrinol Metab 2003;88:2706-13.

283. van Eijk HM, Bloemen JG, Dejong CH. Application of liquid chromatography-mass spectrometry to measure short chain fatty acids in blood. J Chromatogr B Analyt Technol Biomed Life Sci 2009;877:719-24.

284. Goossens GH, Bizzarri A, Venteclef N, et al. Increased adipose tissue oxygen tension in obese compared with lean men is accompanied by insulin resistance, impaired adipose tissue capillarization, and inflammation. Circulation 2011;124:67-76.

285. Lin K, Kools H, de Groot PJ, et al. MADMAX - Management and analysis database for multiple $\sim$ omics experiments. J Integr Bioinform 2011;8:160.

286. Smyth GK. Linear models and empirical bayes methods for assessing differential expression in microarray experiments. Stat Appl Genet Mol Biol 2004;3:Article3.

287. Garcia-Villalba R, Gimenez-Bastida JA, Garcia-Conesa MT, et al. Alternative method for gas chromatography-mass spectrometry analysis of short-chain fatty acids in faecal samples. J Sep Sci 2012;35:1906-13.

288. Bates D, Mächler M, Bolker B, et al. Fitting Linear Mixed-Effects Models Using lme4. Journal of Statistical Software 2015;67.

289. Liaw A, Wiener M. Classification and Regression by randomForest. R News 2002;2:18-22. 
290. Proust-Lima C, Philipps V, Liquet B. Estimation of Extended Mixed Models Using Latent Classes and Latent Processes: the R package lcmm. 2015.

291. Oksanen JFGB KR, Legendre P, Minchin PR, O’Hara RB, Simpson GL et al. . Package 'vegan' version 2.0-2. 2011.

292. Yap IK, Li JV, Saric J, et al. Metabonomic and microbiological analysis of the dynamic effect of vancomycin-induced gut microbiota modification in the mouse. J Proteome Res 2008;7:3718-28.

293. Bech-Nielsen GV, Hansen CH, Hufeldt MR, et al. Manipulation of the gut microbiota in C57BL/6 mice changes glucose tolerance without affecting weight development and gut mucosal immunity. Res Vet Sci 2012;92:501-8

294. Carvalho BM, Guadagnini D, Tsukumo DM, et al. Modulation of gut microbiota by antibiotics improves insulin signalling in high-fat fed mice. Diabetologia 2012;55:2823-34

295. Rune I, Hansen CH, Ellekilde M, et al. Ampicillin-improved glucose tolerance in diet-induced obese C57BL/6NTac mice is age dependent. J Diabetes Res 2013;2013:319321.

296. Rajpal DK, Klein JL, Mayhew D, et al. Selective Spectrum Antibiotic Modulation of the Gut Microbiome in Obesity and Diabetes Rodent Models. PLoS One 2015;10:e0145499.

297. Mikkelsen KH, Frost M, Bahl MI, et al. Effect of Antibiotics on Gut Microbiota, Gut Hormones and Glucose Metabolism. PLoS One 2015;10:e0142352.

298. Thuny F, Richet H, Casalta JP, et al. Vancomycin treatment of infective endocarditis is linked with recently acquired obesity. PLoS One 2010;5:e9074.

299. Million M, Thuny F, Angelakis E, et al. Lactobacillus reuteri and Escherichia coli in the human gut microbiota may predict weight gain associated with vancomycin treatment. Nutr Diabetes 2013;3:e87.

300. Gonzales M, Pepin J, Frost EH, et al. Faecal pharmacokinetics of orally administered vancomycin in patients with suspected Clostridium difficile infection. BMC Infect Dis 2010;10:363.

301. Ridlon JM, Kang DJ, Hylemon PB. Bile salt biotransformations by human intestinal bacteria. Journal of Lipid Research 2006;47:241-259.

302. Brighton CA, Rievaj J, Kuhre RE, et al. Bile acids trigger GLP-1 release predominantly by accessing basolaterally-located G-protein coupled bile acid receptors. Endocrinology 2015:en20151321.

303. Gao Z, Yin J, Zhang J, et al. Butyrate improves insulin sensitivity and increases energy expenditure in mice. Diabetes 2009;58:1509-17.

304. Ganal SC, Sanos SL, Kallfass C, et al. Priming of natural killer cells by nonmucosal mononuclear phagocytes requires instructive signals from commensal microbiota. Immunity 2012;37:171-86.

305. Brestoff JR, Artis D. Commensal bacteria at the interface of host metabolism and the immune system. Nat Immunol 2013;14:676-84.

306. Fukuda S, Toh H, Hase K, et al. Bifidobacteria can protect from enteropathogenic infection through production of acetate. Nature 2011;469:543-7.

307. Furusawa Y, Obata Y, Fukuda S, et al. Commensal microbe-derived butyrate induces the differentiation of colonic regulatory T cells. Nature 2013;504:446-50.

308. $\quad$ McCutcheon AL. Latent class analysis: Sage, 1987.

309. Mikkelsen KH, Knop FK, Frost M, et al. Use of Antibiotics and Risk of Type 2 Diabetes: A Population-Based Case-Control Study. J Clin Endocrinol Metab 2015;100:3633-40

310. Kerr CA, Grice DM, Tran CD, et al. Early life events influence whole-of-life metabolic health via gut microflora and gut permeability. Crit Rev Microbiol 2015;41:326-40.

311. Backhed F, Manchester JK, Semenkovich CF, et al. Mechanisms underlying the resistance to dietinduced obesity in germ-free mice. Proc Natl Acad Sci U S A 2007;104:979-84.

312. Rabot S, Membrez M, Bruneau A, et al. Germ-free C57BL/6J mice are resistant to high-fat-dietinduced insulin resistance and have altered cholesterol metabolism. FASEB J 2010;24:4948-59.

313. Tremaroli V, Backhed F. Functional interactions between the gut microbiota and host metabolism Nature 2012;489:242-9.

314. Karlsson FH, Tremaroli V, Nookaew I, et al. Gut metagenome in European women with normal, impaired and diabetic glucose control. Nature 2013;498:99-103.

315. Ridaura VK, Faith JJ, Rey FE, et al. Gut microbiota from twins discordant for obesity modulate metabolism in mice. Science 2013;341:1241214

316. National Cholesterol Education Program Expert Panel on Detection E, Treatment of High Blood Cholesterol in A. Third Report of the National Cholesterol Education Program (NCEP) Expert Panel on Detection, Evaluation, and Treatment of High Blood Cholesterol in Adults (Adult Treatment Panel III) final report. Circulation 2002;106:3143-421 
317. ter Horst KW, Gilijamse PW, Koopman KE, et al. Insulin resistance in obesity can be reliably identified from fasting plasma insulin. Int J Obes (Lond) 2015;39:1703-9.

318. Rajilić-Stojanović M, Heilig HG, Molenaar D, et al. Development and application of the human intestinal tract chip, a phylogenetic microarray: analysis of universally conserved phylotypes in the abundant microbiota of young and elderly adults. Environmental microbiology 2009;11:1736-1751.

319. Lahti L, Elo LL, Aittokallio T, et al. Probabilistic analysis of probe reliability in differential gene expression studies with short oligonucleotide arrays. IEEE/ACM Trans Comput Biol Bioinform 2011;8:217-25.

320. Sing T, Sander O, Beerenwinkel N, et al. ROCR: visualizing classifier performance in R. Bioinformatics 2005;21:3940-3941.

321. Team RC. R: A language and environment for statistical computing. Vienna, Austria: R Foundation for Statistical Computing, 2015.

322. Zhang X, Shen D, Fang Z, et al. Human gut microbiota changes reveal the progression of glucose intolerance. PLoS One 2013;8:e71108.

323. Dewulf EM, Cani PD, Claus SP, et al. Insight into the prebiotic concept: lessons from an exploratory, double blind intervention study with inulin-type fructans in obese women. Gut 2013;62:1112-21.

324. Vulevic J, Juric A, Tzortzis G, et al. A mixture of trans-galactooligosaccharides reduces markers of metabolic syndrome and modulates the fecal microbiota and immune function of overweight adults. J Nutr 2013;143:324-31.

325. Ghoshal UC, Shukla R, Ghoshal U, et al. The gut microbiota and irritable bowel syndrome: friend or foe? Int J Inflam 2012;2012:151085.

326. Rajilic-Stojanovic M, Biagi E, Heilig HG, et al. Global and deep molecular analysis of microbiota signatures in fecal samples from patients with irritable bowel syndrome. Gastroenterology 2011;141:1792-801.

327. Wang ZK, Yang YS, Chen Y, et al. Intestinal microbiota pathogenesis and fecal microbiota transplantation for inflammatory bowel disease. World J Gastroenterol 2014;20:14805-20.

328. Olsen GJ, Lane DJ, Giovannoni SJ, et al. Microbial ecology and evolution: a ribosomal RNA approach. Annu Rev Microbiol 1986;40:337-65.

329. Lane DJ, Pace B, Olsen GJ, et al. Rapid determination of $16 \mathrm{~S}$ ribosomal RNA sequences for phylogenetic analyses. Proc Natl Acad Sci U S A 1985;82:6955-9.

330. Schmidt TS, Matias Rodrigues JF, von Mering C. Limits to robustness and reproducibility in the demarcation of operational taxonomic units. Environ Microbiol 2014.

331. Drummond C. Replicability is not reproducibility: nor is it good science. Proc. Eval. Methods Mach. Learn. Workshop 26th ICML, Montreal, Quebec, Canada. 2009.

332. Casadevall A, Fang FC. Reproducible science. Infect Immun 2010;78:4972-5.

333. Bokulich NA, Subramanian S, Faith JJ, et al. Quality-filtering vastly improves diversity estimates from Illumina amplicon sequencing. Nat Methods 2013;10:57-9.

334. Cai Y, Sun Y. ESPRIT-Tree: hierarchical clustering analysis of millions of 16S rRNA pyrosequences in quasilinear computational time. Nucleic Acids Res 2011;39:e95.

335. Li W, Godzik A. Cd-hit: a fast program for clustering and comparing large sets of protein or nucleotide sequences. Bioinformatics 2006;22:1658-9.

336. Edgar RC. Search and clustering orders of magnitude faster than BLAST. Bioinformatics 2010;26:2460-1.

337. Mahe F, Rognes T, Quince C, et al. Swarm: robust and fast clustering method for amplicon-based studies. PeerJ 2014;2:e593.

338. Cole JR, Wang Q, Fish JA, et al. Ribosomal Database Project: data and tools for high throughput rRNA analysis. Nucleic Acids Res 2014;42:D633-42.

339. Yilmaz P, Parfrey LW, Yarza P, et al. The SILVA and "All-species Living Tree Project (LTP)" taxonomic frameworks. Nucleic Acids Res 2014;42:D643-8.

340. He Y, Caporaso JG, Jiang XT, et al. Stability of operational taxonomic units: an important but neglected property for analyzing microbial diversity. Microbiome 2015;3:20.

341. Wang Y, Qian PY. Conservative fragments in bacterial 16S rRNA genes and primer design for 16S ribosomal DNA amplicons in metagenomic studies. PLoS One 2009;4:e7401.

342. He Y, Zhou BJ, Deng GH, et al. Comparison of microbial diversity determined with the same variable tag sequence extracted from two different PCR amplicons. BMC Microbiol 2013;13:208.

343. Clooney AG, Fouhy F, Sleator RD, et al. Comparing Apples and Oranges?: Next Generation Sequencing and Its Impact on Microbiome Analysis. PLoS One 2016;11:e0148028. 
344. Gilbert JA, Jansson JK, Knight R. The Earth Microbiome project: successes and aspirations. BMC Biol 2014;12:69.

345. Engelbrektson A, Kunin V, Wrighton KC, et al. Experimental factors affecting PCR-based estimates of microbial species richness and evenness. ISME J 2010;4:642-7.

346. May A, Abeln S, Crielaard W, et al. Unraveling the outcome of 16S rDNA-based taxonomy analysis through mock data and simulations. Bioinformatics 2014.

347. Tremblay J, Singh K, Fern A, et al. Primer and platform effects on 16S rRNA tag sequencing. Front Microbiol 2015;6:771.

348. Barb JJ, Oler AJ, Kim HS, et al. Development of an Analysis Pipeline Characterizing Multiple Hypervariable Regions of 16S rRNA Using Mock Samples. PLoS One 2016;11:e0148047.

349. Koskinen K, Auvinen P, Bjorkroth KJ, et al. Inconsistent Denoising and Clustering Algorithms for Amplicon Sequence Data. J Comput Biol 2015;22:743-51.

350. Caporaso JG, Kuczynski J, Stombaugh J, et al. QIIME allows analysis of high-throughput community sequencing data. Nat Methods 2010;7:335-6.

351. Degnan PH, Ochman H. Illumina-based analysis of microbial community diversity. ISME J 2011

352. Schirmer M, Ijaz UZ, D'Amore R, et al. Insight into biases and sequencing errors for amplicon sequencing with the Illumina MiSeq platform. Nucleic Acids Res 2015;43:e37.

353. Lozupone C, Knight R. UniFrac: a new phylogenetic method for comparing microbial communities. Appl Environ Microbiol 2005;71:8228-35.

354. Hong SH, Bunge J, Jeon SO, et al. Predicting microbial species richness. Proc Natl Acad Sci U S A 2006;103:117-22.

355. Faith DP. The role of the phylogenetic diversity measure, PD, in bio-informatics: getting the definition right. Evol Bioinform Online 2006;2:277-83.

356. Marchesi JR, Adams DH, Fava F, et al. The gut microbiota and host health: a new clinical frontier. Gut 2016;65:330-9.

357. Van de Wiele T, Vanhaecke L, Boeckaert C, et al. Human colon microbiota transform polycyclic aromatic hydrocarbons to estrogenic metabolites. Environ Health Perspect 2005;113:6-10.

358. Van de Wiele TR, Verstraete W, Siciliano SD. Polycyclic aromatic hydrocarbon release from a soil matrix in the in vitro gastrointestinal tract. J Environ Qual 2004;33:1343-53.

359. Sivieri K, Morales ML, Saad SM, et al. Prebiotic effect of fructooligosaccharide in the simulator of the human intestinal microbial ecosystem (SHIME(R) model). J Med Food 2014;17:894-901.

360. Aguirre M, Jonkers DM, Troost FJ, et al. In vitro characterization of the impact of different substrates on metabolite production, energy extraction and composition of gut microbiota from lean and obese subjects. PLoS One 2014;9:e113864.

361. Payne AN, Zihler A, Chassard C, et al. Advances and perspectives in in vitro human gut fermentation modeling. Trends Biotechnol 2012;30:17-25.

362. Kovatcheva-Datchary P, Egert M, Maathuis A, et al. Linking phylogenetic identities of bacteria to starch fermentation in an in vitro model of the large intestine by RNA-based stable isotope probing. Environ Microbiol 2009;11:914-26.

363. McDonald JA, Schroeter K, Fuentes S, et al. Evaluation of microbial community reproducibility, stability and composition in a human distal gut chemostat model. J Microbiol Methods 2013;95:16774.

364. Maathuis AJH, van den Heuvel EG, Schoterman MHC, et al. Galacto-Oligosaccharides Have Prebiotic Activity in a Dynamic in vitro Colon Model Using a C-Labeling Technique. J Nutr 2012;142:12051212.

365. Maathuis A, Hoffman A, Evans A, et al. The effect of the undigested fraction of maize products on the activity and composition of the microbiota determined in a dynamic in vitro model of the human proximal large intestine. J Am Coll Nutr 2009;28:657-66.

366. Aguirre M, Ramiro-Garcia J, Koenen ME, et al. To pool or not to pool? Impact of the use of individual and pooled fecal samples for in vitro fermentation studies. J Microbiol Methods 2014;107C:1-7.

367. de Graaf AA, Maathuis A, de Waard P, et al. Profiling human gut bacterial metabolism and its kinetics using [U-13C]glucose and NMR. NMR Biomed 2010;23:2-12.

368. van Nuenen MHMC, Diederick Meyer P, Venema K. The Effect of Various Inulins and Clostridium difficile on the Metabolic Activity of the Human Colonic Microbiota in vitro. Microb Ecol Health Dis 2003;15:137-144. 
369. Ramiro-Garcia J HG, Giatsis C, Sipkema D, Zoetendal E, Schaap P \& Smidt H. NG-Tax, a highly accurate and validated pipeline for analysis of $16 \mathrm{~S}$ rRNA amplicons from complex biomes [version 1; referees: awaiting peer review]. F1000Research 2016.

370. Zoetendal EG, Booijink CC, Klaassens ES, et al. Isolation of RNA from bacterial samples of the human gastrointestinal tract. Nat Protoc 2006;1:954-9.

371. Murphy NR, Hellwig RJ. Improved nucleic acid organic extraction through use of a unique gel barrier material. Biotechniques 1996;21:934-6, 938-9.

372. Kopylova E, Noe L, Touzet H. SortMeRNA: fast and accurate filtering of ribosomal RNAs in metatranscriptomic data. Bioinformatics 2012;28:3211-7.

373. Robinson MD, McCarthy DJ, Smyth GK. edgeR: a Bioconductor package for differential expression analysis of digital gene expression data. Bioinformatics 2010;26:139-40.

374. Tabernero M, Venema K, Maathuis AJ, et al. Metabolite production during in vitro colonic fermentation of dietary fiber: analysis and comparison of two European diets. J Agric Food Chem 2011;59:8968-75

375. Lozupone C, Lladser ME, Knights D, et al. UniFrac: an effective distance metric for microbial community comparison. ISME J 2011;5:169-72

376. Macfarlane GT, Macfarlane S. Models for intestinal fermentation: association between food components, delivery systems, bioavailability and functional interactions in the gut. Curr Opin Biotechnol 2007;18:156-62.

377. Van den Abbeele P, Grootaert C, Marzorati M, et al. Microbial community development in a dynamic gut model is reproducible, colon region specific, and selective for Bacteroidetes and Clostridium cluster IX. Appl Environ Microbiol 2010;76:5237-46.

378. Minekus M, Smeets-Peeters M, Bernalier A, et al. A computer-controlled system to simulate conditions of the large intestine with peristaltic mixing, water absorption and absorption of fermentation products. Appl Microbiol Biotechnol 1999;53:108-14.

379. Gao K, Xu A, Krul C, et al. Of the major phenolic acids formed during human microbial fermentation of tea, citrus, and soy flavonoid supplements, only 3,4-dihydroxyphenylacetic acid has antiproliferative activity. J Nutr 2006;136:52-7.

380. Martinez RC, Cardarelli HR, Borst W, et al. Effect of galactooligosaccharides and Bifidobacterium animalis Bb-12 on growth of Lactobacillus amylovorus DSM 16698, microbial community structure, and metabolite production in an in vitro colonic model set up with human or pig microbiota. FEMS Microbiol Ecol 2013;84:110-23.

381. Rose DJ, Venema K, Keshavarzian A, et al. Starch-entrapped microspheres show a beneficial fermentation profile and decrease in potentially harmful bacteria during in vitro fermentation in faecal microbiota obtained from patients with inflammatory bowel disease. Br J Nutr 2010;103:1514-24.

382. Leijdekkers AGM, Aguirre M, Venema K, et al. In Vitro Fermentability of Sugar Beet Pulp Derived Oligosaccharides Using Human and Pig Fecal Inocula. J Agric Food Chem 2014;62:1079-1087.

383. Macfarlane GT, Gibson GR, Cummings JH. Comparison of fermentation reactions in different regions of the human colon. J Appl Bacteriol 1992;72:57-64.

384. Russell WR, Gratz SW, Duncan SH, et al. High-protein, reduced-carbohydrate weight-loss diets promote metabolite profiles likely to be detrimental to colonic health. Am J Clin Nutr 2011;93:1062 72.

385. Ouwehand AC, Derrien M, de Vos W, et al. Prebiotics and other microbial substrates for gut functionality. Curr Opin Biotechnol 2005;16:212-7.

386. Aguirre M, Eck A, Koenen ME, et al. Diet drives quick changes in the metabolic activity and composition of human gut microbiota in a validated in vitro gut model. Res Microbiol 2015;167:11425 .

387. Feria-Gervasio D, Denis S, Alric M, et al. In vitro maintenance of a human proximal colon microbiota using the continuous fermentation system P-ECSIM. Appl Microbiol Biotechnol 2011;91:1425-33.

388. Aguirre M, Eck A, Koenen ME, et al. Evaluation of an optimal preparation of human standardized fecal inocula for in vitro fermentation studies. J Microbiol Methods 2015;117:78-84.

389. Rajilic-Stojanovic M, Maathuis A, Heilig HG, et al. Evaluating the microbial diversity of an in vitro model of the human large intestine by phylogenetic microarray analysis. Microbiology 2010;156:327081.

390. Egert M, de Graaf AA, Maathuis A, et al. Identification of glucose-fermenting bacteria present in an in vitro model of the human intestine by RNA-stable isotope probing. FEMS Microbiol Ecol 2007;60:126-35. 
391. David LA, Maurice CF, Carmody RN, et al. Diet rapidly and reproducibly alters the human gut microbiome. Nature 2014;505:559-63.

392. Wu GD, Chen J, Hoffmann C, et al. Linking Long-Term Dietary Patterns with Gut Microbial Enterotypes. Science 2011;334:105-108.

393. Ze X, Duncan SH, Louis P, et al. Ruminococcus bromii is a keystone species for the degradation of resistant starch in the human colon. ISME J 2012;6:1535-43.

394. Taras D, Simmering R, Collins MD, et al. Reclassification of Eubacterium formicigenerans Holdeman and Moore 1974 as Dorea formicigenerans gen. nov., comb. nov., and description of Dorea longicatena sp. nov., isolated from human faeces. Int J Syst Evol Microbiol 2002;52:423-8.

395. Matijasic BB, Obermajer T, Lipoglavsek L, et al. Association of dietary type with fecal microbiota in vegetarians and omnivores in Slovenia. Eur J Nutr 2014;53:1051-64.

396. Zhou L, Fang L, Sun Y, et al. Effects of the dietary protein level on the microbial composition and metabolomic profile in the hindgut of the pig. Anaerobe 2016;38:61-9.

397. Yang J, Martinez I, Walter J, et al. In vitro characterization of the impact of selected dietary fibers on fecal microbiota composition and short chain fatty acid production. Anaerobe 2013;23:74-81.

398. Walker AW, Ince J, Duncan SH, et al. Dominant and diet-responsive groups of bacteria within the human colonic microbiota. The ISME journal 2011;5:220-230.

399. O'Keefe SJ, Li JV, Lahti L, et al. Fat, fibre and cancer risk in African Americans and rural Africans. Nat Commun 2015;6:6342.

400. Murphy EF, Cotter PD, Hogan A, et al. Divergent metabolic outcomes arising from targeted manipulation of the gut microbiota in diet-induced obesity. Gut 2012.

401. Bashan A, Gibson TE, Friedman J, et al. Universality of human microbial dynamics. Nature 2016;534:259-62.

402. Kaiser JC, Verschoor CP, Surette MG, et al. Host cytokine responses distinguish invasive from airway isolates of the Streptococcus milleri/anginosis group. BMC Infect Dis 2014; 14:498.

403. Faust K, Raes J. Host-microbe interaction: Rules of the game for microbiota. Nature 2016;534:182-3

404. McClellan J, King MC. Genetic heterogeneity in human disease. Cell 2010;141:210-7.

405. Nature's Services: Societal Dependence on Natural Ecosystems: Island Press, 1997.

406. Velagapudi VR, Hezaveh R, Reigstad CS, et al. The gut microbiota modulates host energy and lipid metabolism in mice. J Lipid Res 2010;51:1101-12.

407. Cani PD, Neyrinck AM, Fava F, et al. Selective increases of bifidobacteria in gut microflora improve high-fat-diet-induced diabetes in mice through a mechanism associated with endotoxaemia. Diabetologia 2007;50:2374-83.

408. Samuel BS, Shaito A, Motoike T, et al. Effects of the gut microbiota on host adiposity are modulated by the short-chain fatty-acid binding $\mathrm{G}$ protein-coupled receptor, Gpr41. Proc Natl Acad Sci U S A 2008;105:16767-72.

409. Samuel BS, Gordon JI. A humanized gnotobiotic mouse model of host-archaeal-bacterial mutualism Proc Natl Acad Sci U S A 2006;103:10011-6.

410. Tautz D, Domazet-Loso T. The evolutionary origin of orphan genes. Nat Rev Genet 2011;12:692-702.

411. Ballal SA, Gallini CA, Segata N, et al. Host and gut microbiota symbiotic factors: lessons from inflammatory bowel disease and successful symbionts. Cell Microbiol 2011;13:508-17.

412. Konstantinidis KT, Tiedje JM. Genomic insights that advance the species definition for prokaryotes. Proc Natl Acad Sci U S A 2005;102:2567-72.

413. Hayashi T, Makino K, Ohnishi M, et al. Complete genome sequence of enterohemorrhagic Escherichia coli O157:H7 and genomic comparison with a laboratory strain K-12. DNA Res 2001;8:11-22.

414. Jacobi CA, Malfertheiner P. Escherichia coli Nissle 1917 (Mutaflor): new insights into an old probiotic bacterium. Dig Dis 2011;29:600-7.

415. Kelly BJ, Gross R, Bittinger K, et al. Power and sample-size estimation for microbiome studies using pairwise distances and PERMANOVA. Bioinformatics 2015;31:2461-2468.

416. Stammler F, Glasner J, Hiergeist A, et al. Adjusting microbiome profiles for differences in microbial load by spike-in bacteria. Microbiome 2016;4:28.

417. Wallace BD, Wang H, Lane KT, et al. Alleviating cancer drug toxicity by inhibiting a bacterial enzyme. Science 2010;330:831-5. 


\section{Co-author affiliations}

Hauke Smidt ${ }^{\mathrm{a}, \mathrm{b}}$

Erwin G Zoetendal ${ }^{\mathrm{a}, \mathrm{b}}$

Javier Ramiro-Garcia ${ }^{a, b, c}$

Peter J Schaap ${ }^{\circ}$

Catherine A. Mbakwa a,d

John Penders d,e

Paul H. M. Savelkoul ${ }^{\mathrm{e}}$

Carel Thijs ${ }^{\mathrm{d}}$

Pieter C. Dagnelie ${ }^{\mathrm{d}, \mathrm{f}}$

Monique Mommers ${ }^{\mathrm{d}}$

Ilja C.W. Arts d,f,g

Dorien Reijnders a,h

Gijs H. Goossens ${ }^{\text {a,h }}$

Evelien P.J.G. Neis ${ }^{\text {a,i }}$

Christina M. van der Beek ${ }^{\mathrm{a}, \mathrm{i}}$

\author{
Jasper Most ${ }^{\mathrm{h}}$ \\ Jens J. Holst ${ }^{j}$ \\ Kaatje Lenaerts a,i \\ Ruud S. Kootte ${ }^{\mathrm{a}, \mathrm{k}}$ \\ Max Nieuwdorp a,k \\ Albert K. Groen ${ }^{\mathrm{a}, \mathrm{m}}$ \\ Steven W.M. Olde Damink ${ }^{\mathrm{i}, \mathrm{n}}$ \\ Mark V. Boekschoten ${ }^{\mathrm{a}, \mathrm{o}}$ \\ Cornelis H.C. Dejong a, ${ }^{\mathrm{a}}$ \\ Ellen E. Blaak ${ }^{\mathrm{a}, \mathrm{h}}$ \\ Christos Giatsis $^{p}$ \\ Detmer Sipkema ${ }^{\text {b }}$
}

${ }^{a}$ TI Food and Nutrition (TIFN) P.O. Box 557, 6700 AN, The Netherlands

${ }^{b}$ Laboratory of Microbiology, Wageningen University, 6708 WE, Wageningen, The Netherlands

${ }^{\mathrm{c}}$ Laboratory of Systems and Synthetic Biology, Wageningen University, $6708 \mathrm{WE}$, Wageningen, The Netherlands

${ }^{\mathrm{d}}$ Department of Epidemiology, Maastricht University, CAPHRI School for Public Health and Primary Care, 6200 MD, Maastricht, The Netherlands.

${ }^{\mathrm{e}}$ Department of Medical Microbiology, Maastricht University Medical Centre, NUTRIM School for Nutrition, Toxicology and Metabolism, Maastricht, The Netherlands.

${ }^{\mathrm{f}}$ Department of Epidemiology, Maastricht University, CARIM School for Cardiovascular Diseases, Maastricht, The Netherlands.

${ }^{\mathrm{g}}$ Maastricht Centre for Systems Biology (MaCSBio), Maastricht, The Netherlands.

${ }^{\mathrm{h}}$ Department of Human Biology, NUTRIM School of Nutrition and Translational Research in Metabolism, Maastricht University Medical Center+, 6229 ER Maastricht, The Netherlands.

${ }^{\mathrm{i}}$ Department of Surgery, NUTRIM School of Nutrition and Translational Research in Metabolism, Maastricht University Medical Center+, 6229 ER Maastricht, The Netherlands.

${ }^{\mathrm{j}}$ NNF Center for Basic Metabolic Research, Department of Biomedical Sciences, The Panum Institute, University of Copenhagen, 2200 Copenhagen, Denmark.

${ }^{\mathrm{k}}$ Department of Vascular Medicine and Department of Internal Medicine, University of Amsterdam, 1100 DD Amsterdam, The Netherlands.

${ }^{\mathrm{m}}$ Department of Pediatric Gastroenterology and Hepatology, Beatrix Children's Hospital, University Medical Center Groningen, 9713 GZ Groningen, The Netherlands.

${ }^{\mathrm{n}}$ Department of HPB Surgery and Liver Transplantation, Institute of Liver and Digestive Health, University College London, London, United Kingdom.

${ }^{\circ}$ Nutrition, Metabolism and Genomics group, Division of Human Nutrition, Wageningen University, 6700EV Wageningen, The Netherlands

p Aquaculture and Fisheries Group, Wageningen University, 6708 WD Wageningen, The Netherlands 


\section{Acknowledgements - Dankwoord}

The beginning of my $\mathrm{PhD}$ was dominated by Murphies Law, which made it a difficult period for me but also, or even more so, for the people who had to deal with me. This booklet was really is a team effort and also feels like a bit of closure for this period, so with this section I'd like to thank anyone who has directly or indirectly contributed to this thesis.

Hauke, bedankt voor je toewijding aan de promotor taken en het altijd kritisch lezen van mijn manuscripten. Ze werden er altijd instantly beter door. Doordat we in drie verschillende TIFN projecten zaten waar iedereen per project van $100 \%$ inzet uitging was het niet altijd gemakkelijk om dat waar te maken, maar gelukkig heb je ons met je altijd diplomatieke aanpak redelijk uit de wind kunnen houden en het was pretting om je daarom in onze hoek te hebben. Erwin, ook jij bedankt voor je begeleiding, enthousiasme, discussies en kennis die je hebt omdat je vanaf het begin in dit mooie veld aanwezig bent geweest. Ik vergeet zeker niet de mooie gesprekken en updates over je jeugdhelden uit de jaren 80 thrash scene. Een genre wat ik overigens ook zeer kan waarderen. Maar beide vooral bedankt voor het begrip toen het op persoonlijk vlak niet zo goed ging. Deze steun heeft me heel veel goed gedaan en maakte andere dingen daardoor lichter.

Javi 'Habi', we have more names for you, but I cannot mention those here. We started our PhD projects only a few months apart in the same project(s). It's not easy being $100 \%$ in three different projects, especially at our age ;). We had to deal with the same... stuff. 'Gedeelde smart is halve smart' as we say. You'll probably have a nice Spanish saying in return. But next to being in the situation we have similar way approaching our work (the devil's in the details, thanks Hauke and Erwin for your patience with us). Thanks for the discussions and walks to the Albert Heijn for lunch, for sharing your look on life and for the one liners such as 'we are a screw' and insert whatever verb 'like hell' and of course the (Spanish) road trips! It's been a pleasure and 'Muchos buena e gracias', so how's your Dutch after 5 years? Romy 'Womy', we've been roommates for about three years and during a $\mathrm{PhD}$ that seems like a lifetime. It's been inspiring to see how you deal with everything that came your way. It's also been inspiring to have seen you grow as a person as well as a scientist. Take it as a compliment that I still think that you have 3 years to go (it means you don't have that 'only a few months to go look' ;) ). I'm really proud and thankful that you are my paranymphs.

Voordat ik naar de microbiologen ga, wil ik eerst de supporting staff bedanken. Zonder jullie geen laboratory of microbiology. Anja \& Carolien - bedankt voor het geduld, hulp en begrip, Sjon, Steven'(s)', Ton - verjaardagscake van de markt, Tom vd W - RWC 2011 streamen, Tom S, Monika, Gosse, Wim, Jannie, bedankt! En speciaal het HITChip team, Ineke - thanks voor de goede gesprekken en je hulp, komt allemaal goed met de jongens :), Wilma, Philippe, Jorn, Pien en Hans - rustpunt in het lab an all around good guy, bedankt 
voor de goede gesprekken. Bedankt voor alle hulp met de vele HITChips, maar meer nog voor de goede sfeer in het lab, wat erg prettig is als je een paar honderd keer hetzelfde moet doen.

A big thanks to all my roomies in the basement, Janneke 'Akkermans', Lennart 'Lennie', Romy, 'Koendjiebiharie', Oylum, Peng, Iannis, and Leo. Thanks for sharing work and nonwork stuff. A special thanks to Leo 'moi', although I sometimes might have proclaimed this, after I made something work after weeks of frustration, you are the true "king of R". Thank you so much for your patience and explanations, your help was invaluable. Also besides work we had some great discussions on life, during and after a dark period in mine. Thanks for those.

Of course a thank you to all the MolEco's and especially, Susana - thanks for the conversations and help with COMIC, Noora, Tomas, Tom vdB \& Sebastian - beide altijd super behulpzaam en bedankt voor de stammen voor de MCs, Coline, Teresita, Dennis 'Denise', Aleksandr 'The Rrrusian', Naim, Gianina, Klaudyna, Hanne, Clara, Detmer, Georg, Inra, Teresita, Loowee, Johanna, Joan, Floor, Detmer, Sudarshan, Maria S, Hanne, Hugo, Kees, Mauricio and Marcelle.

Overall I had a great time at microbiology and thank you Willem for creating such a stimulating environment. The international character spiced things up, especially at lunch. So I would like to thank all microbiologists and SSB'ers for such an overall great atmosphere. A few years ago after he graduated someone told me that they really appreciated something small I had said a few years prior. I hadn't realized this had such an impact, but in the last few years I've come to appreciate those small things and sometimes not so small things. So a special thanks to, Tijn, Melvin, Daan, Aylin, Nico, Elleke, Nicolas 'Strepsils', Irene, Sven, Teunke, Bas, Raymond, Benoit 'Benoise', Bastian 'Basti', Kal, Maarten, Jesse, Wiebe, Catalina, Emma, Rob, Jasper, Maria SD, Bart, Monika, Becca, Monir,Mark D, and Derya for your contributions (conscious or otherwise) to this thesis.

Of course a big thank you to all of the co-authors as most of the work presented here wouldn't have been possible without you. I would like to apologize, like with sports I sometimes turn into a different person during work and thank you for coping with this and your patience. In particular, Catherine, for your positive outlook and for (maybe unconsciously) putting things in perspective for me. I have so much respect for your perseverance in your situation. Dorien, it was a pleasure working with you, good luck in the USA. Marisol, thanks for creating such a nice working atmosphere, good luck with finding a Postdoc. Ruud - sfeermaker tijden de TIFN meetings en Ellen, Gijs, Ilja, John en Koen, bedankt voor alle positieve input. Same goes for my students, Kerstin, Jingjie, Jiaxing and Dominic; couldn't have done it without your help. And thanks to my former colleagues Amra, Tiemen 'Tiemus', Corina, Jolanda, Jeroen vBH and Rocio - muchas gracias, you were a big catalyst for this $\mathrm{PhD}$ plan of mine. 
Zonder de hulp van mn vrienden waren was het afgelopen jaren niet goed gegaan. I owe you guys a lot! Bas 'La Laz', Neil 'Nelis' - bedankt voor alles, woorden zijn niet genoeg. Ik herinner me bv nog een middag in Zetten... De T-30 crew; Raymond 'Rievit' - thanks voor alle steun de afgelopen jaren en je positivisme en samen met Elke voor jullie gastvrijheid. Het voelt als vakantie een dagje bij jullie. Kitty, Geerdink, Elsa en Elshof, bedankt voor alle afleiding, steun en na een avondje losgaan met jullie kan een mens er weer tegen ;). De wat actievere leden van Tour de ledig erf voor sportieve afleiding, Emiel Wouter \& Rievit, bedankt voor het afzien, door een paar uur alleen naar een achterwiel te staren vergeet je die PhD ook eens. En verder nog Peter, Stephan, Marie, Paula, Boris, Basje en Dorian, bedankt voor alles wat jullie deze periode voor me betekend hebben.

En natuurlijk de mensen die het dichtstbij hebben gestaan. Mama en Owen, het zijn tropenjaren geweest de afgelopen tijd, maar daardoor is er des te meer waardering voor de mooie dingen en dat ik jullie heb. Ik was tijdens de moeilijke periodes niet altijd even gemakkelijk, dus heel erg bedankt voor de blijvende steun. Ik denk vaker aan jullie dan ik laat merken. Verdere familie, zus, ooms, tantes, neefjes en nichtjes, bedankt voor alles.

$\mathrm{Pa}$, deze is ook voor jou.

And of course I haven't forgotten you, but your name might have slipped my mind, so sorry for that and thanks to you too!

\section{Gerben}




\begin{abstract}
About the author
Gerben Hermes was born on the $16^{\text {th }}$ of April 1981 in Utrecht, The Netherlands. After completing his secondary education at St Gregorius College in Utrecht in 1999, he continued his education with the master Food Technology at Wageningen University. He did his minor thesis in the lab of food chemistry on the identification and cloning of proteases/peptidases from Bifidobacterium spp. and his major thesis in the lab of food microbiology on the growth characteristics under stress of Enterobacter Sakazakii (whose presence in dried infant formulae was associated with infant death) compared to other Enterobacteriaceae whose presence was associated with the same production environment. He concluded his study with internships at the University of Campinas (Unicamp) in Brazil working on the biocompatibility of solvents in the production of bioethanol and Abott in Zwolle, The Netherlands on the evaluation of their existing and development of new sterilisation protocols. After obtaining his Master's degree in 2006 he started working in industry. However after one year at HJ Heinz and three years at Danone research as a jr scientist in the group of microbiology and gut biology he went back to academia and started his in $\mathrm{PhD}$ in 2011 at the laboratory of Microbiology under the supervision of Prof. dr. Hauke Smidt and dr. Erwin Zoetendal. His research focused on gut microbial biomarkers for gastro-intestinal and metabolic homeostasis in humans. The results are presented in this thesis. Currently he is working as a postdoc at the laboratory of Microbiology where he is involved in a project on acetate as metabolic target in the prevention of overweight-related chronic metabolic disorders.
\end{abstract}




\section{List of publications}

Molecular ecological tools to decipher the role of our microbial mass in obesity.

GDA Hermes, EG Zoetendal, H Smidt (2015) Beneficial Microbes 6:61-81.

NG-Tax, a highly accurate and validated pipeline for the analysis of 16S rRNA amplicons from complex biomes.

J Ramiro-Garcia*, GDA Hermes*, C Giatsis, D Sipkema, EG Zoetendal, PJ Schaap, H Smidt submitted

Limited, individual- and cohort-specific patterns of gut microbiota composition associated with tissuespecific insulin sensitivity in overweight and obese males.

GDA Hermes*, D Reijnders*, RS Kootte, GH Goossens, H Smidt, M Nieuwdorp, EE Blaak*, EG Zoetendal* submitted

Gut microbiota and body weight in healthy school-aged children: The KOALA Birth Cohort Study.

CA. Mbakwa*, GDA Hermes*, John Penders, Paul H. M. Savelkoul, Carel Thijs, Pieter C. Dagnelie, Monique Mommers, Erwin G. Zoetendal, Hauke Smidt and Ilja C.W. Arts submitted

Effects of Gut Microbiota Manipulation by Antibiotics on Host Metabolism in Obese Humans: A Randomized Double-Blind Placebo-Controlled Trial.

D Reijnders* GH Goossens*, GDA Hermes, EPJG Neis, CM van der Beek, J Most, JJ Holst, K Lenaerts, RS. Kootte, M Nieuwdorp, AK Groen, SWM Olde Damink, MV Boekschoten, H Smidt, EG Zoetendal, CHC Dejong, EE Blaak (2016) Cell Metabolism 24:63-74.

The gut microbiota and host health: a new clinical frontier.

JR Marchesi, DH Adams, F Fava, GDA Hermes, GM Hirschfield, G Hold, M Quraishi, J Kinross, H Smidt, KM Tuohy, V Thomas, EG Zoetendal, A Hart (2016) Gut 65:330-9.

Cohort profile: LifeLines DEEP, a prospective, general population cohort study in the northern Netherlands: study design and baseline characteristics.

EF Tigchelaar, A Zhernakova, JAM Dekens, GDA Hermes, A Baranska, Z Mujagic, MA Swertz, AM Muñoz, P Deelen, MC Cénit, L Franke, S Scholtens, RP Stolk, C Wijmenga, EJM Feskens (2015) BMJ open 5 (8), $\mathrm{e} 006772$

Twelve-week supplementation of galactooligosaccharide increased bifidobacteria, but did not alter peripheral insulin sensitivity in obese prediabetic men and women, a randomized, placebo-controlled trial

EE Canfora*,CM van der Beek*, GDA Hermes, K Lenaerts, JWE Jocken, GH Goossens, JJ Holst, H Smidt, EG Zoetendal, CHC Dejong, EE Blaak Manuscript in preparation 


\section{Overview of completed training activities}

\section{Discipline specific activities}

\section{$\underline{\text { Courses }}$}

Functional Metagenomics of the Intestinal Tract and Food-Related

Microbes, Helsinki, FI

Advanced visualisation, integration and biological interpretation

of -omics data, Wageningen, NL

TIFN Workshop on Intellectual Property, Wageningen, NL

The intestinal microbiome And Diet in Human and Animal health,

Wageningen, $\mathrm{NL}$ - poster presentation

\section{Meetings}

13th Gut day symposium, Wageningen, NL - poster presentation 2011

8th joint INRA - ROWETT symposium 'Gut microbiota: friend or foe?', 2012

Clermont-Ferrand, FR - oral \& poster presentation

16th Gut day symposium, Amsterdam, NL - poster presentation 2014

17th Gut day symposium, Rotterdam, NL - poster presentation 2015

\section{General courses}

TIFN team workshops, Wageningen, NL 2011

Introductory course 'R', Wageningen, NL 2011

$\begin{array}{ll}\mathrm{PhD} \text { competence assessment, Wageningen, NL } & 2013\end{array}$

Effective behaviour in your Professional surroundings, Wageningen, NL 2013

TIFN WE days, Ameland, NL 2011

TIFN WE days, Apeldoorn, NL 2014

TIFN presentation course: How to get the right message across, Ede, NL 2014

\section{Optionals}

Preparing PhD proposal $\quad 2011$

Laboratory of Microbiology PhD trip, USA \& Canada 2013

Laboratory of Microbiology PhD/Postdoc meetings 2011-2015

Molecular Ecology group meetings $\quad$ 2011-2015

TIFN meetings and colloquia $\quad$ 2011-2015 
The studies presented in this thesis were performed within the framework of TI Food and Nutrition.

\section{Colophon}

Cover design: Iliana Boshoven-Gkini, AgileColor, Noord-Scharwoude, The Netherlands Layout design: Gerben Hermes

Printed by: $\quad$ GVO drukkers \& vormgevers, Ede, The Netherlands 